\title{
Mechanisms of Vascular Smooth Muscle Contraction and the Basis for Pharmacologic Treatment of Smooth Muscle Disorders
}

\author{
F.V. Brozovich, C.J. Nicholson, C.V. Degen, Yuan Z. Gao, M. Aggarwal, and K.G. Morgan
}

Department of Health Sciences, Boston University, Boston, Massachusetts (C.J.N., Y.Z.G., M.A., K.G.M.); Department of Medicine, Mayo Clinic, Rochester, Minnesota (F.V.B.); and Paracelsus Medical University Salzburg, Salzburg, Austria (C.V.D.)

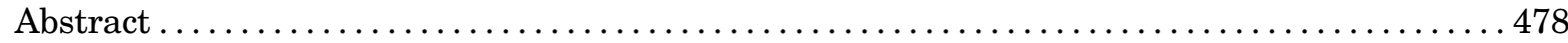

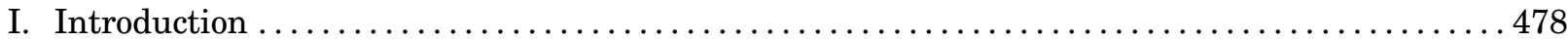

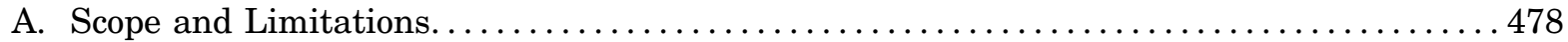

B. Overview of Regulation of Blood Pressure/Vascular Tone..................... 478

1. Guyton View of Regulation Blood Pressure, Kidney Role, Volume Regulation. ....... 478

2. Recent Direct Confirmation of Changes in Vascular Tone/Resistance Related to Changes in Systemic Vascular Resistance and Blood Pressure and the Importance of Vascular Smooth Muscle Contraction in both Normal

Physiology and Pathophysiology-Hypertension......................... 479

3. Racial Differences/Personalized Medicine. ............................... 479

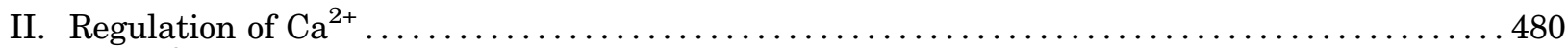

A. $\mathrm{Ca}^{2+}$ Determines Vascular Smooth Muscle Cell Contractility and Phenotype.........480

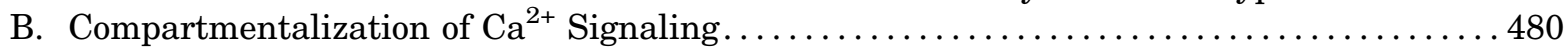

1. $\mathrm{Ca}^{2+}$ Sparklets. ................................................... 480

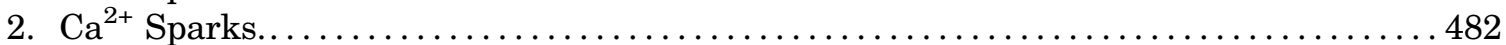

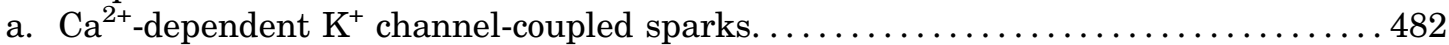

b. $\mathrm{Ca}^{2+}$ gated $\mathrm{Cl}^{-}$channel-coupled sparks........................... 483

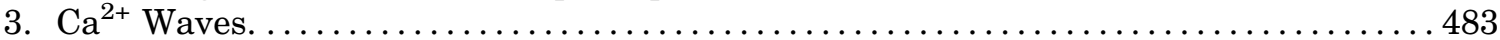

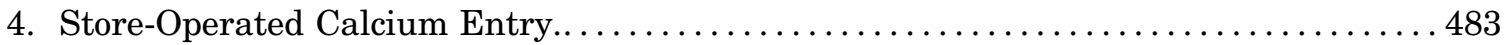

C. Excitation-Transcription Coupling .................................... 484

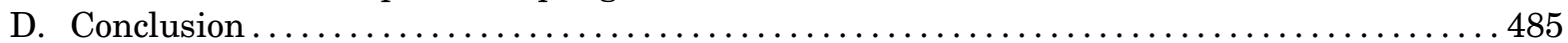

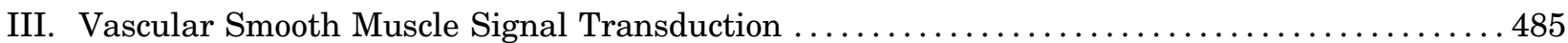

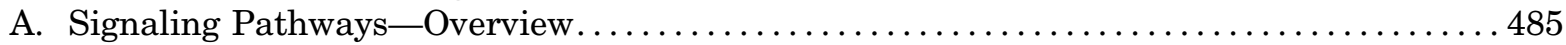

1. Major Pathways Leading to Changes in the Activity of Smooth Muscle Myosin. .... . 485

2. Pathways Leading to Changes in Actin Availability for Interaction with Myosin. ... . 487

3. Tyrosine Phosphorylation of Smooth Muscle Proteins. .................... 488

4. Calcium Sensitization of the Contractile Apparatus. .......................... 488

B. Subcellular Spatial Organization of Signaling Pathways ...................... 488

1. Extracellular Regulated Kinase Scaffolds (Calponin, SmAV, Paxillin, Caveolin,

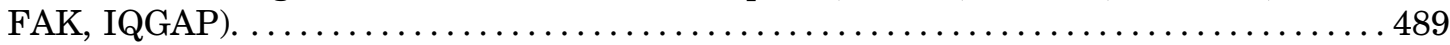

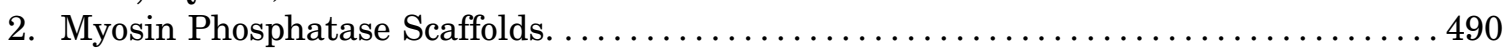

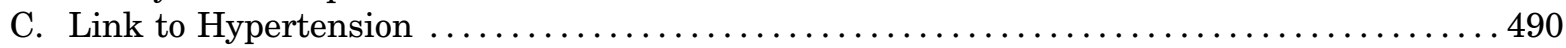

D. Potential Novel Therapeutic Targets/Approaches/Critical Analysis of

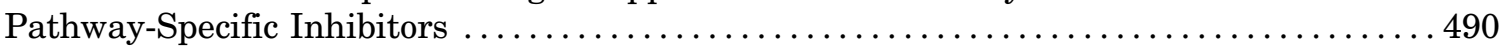

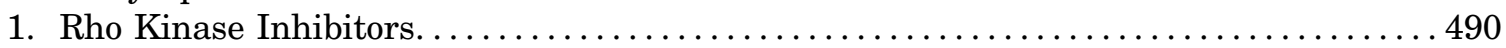

2. Endothelin Inhibitors. .......................................... 490

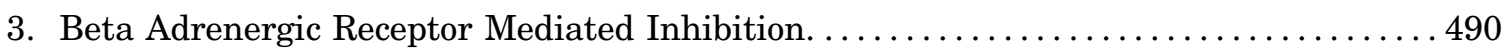

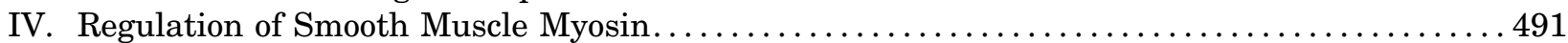

\footnotetext{
Address correspondence to: Kathleen G. Morgan, Department of Health Sciences, Boston University, Boston MA 02215. E-mail: kmorgan@bu.edu dx.doi.org/10.1124/pr.115.010652.
} 
A. Overview of Regulation of the Smooth Muscle Actomyosin ATPase and 20kda light chain Phosphorylation/Smooth Muscle Activation ........................... 491

B. Guanine Nucleotide Exchange Factor Signaling, Rac/Rho, and Analysis of Inhibitors ... 492

C. Phenotypic Switching of Contractile Proteins during Development and Disease:

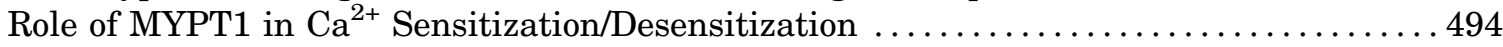

1. Smooth Muscle Myosin Heavy Chain. ................................ 494

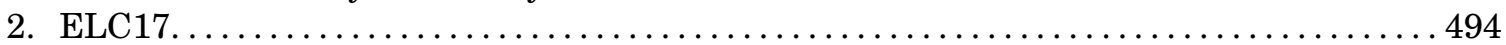

3. MYPT1. .................................................... 495

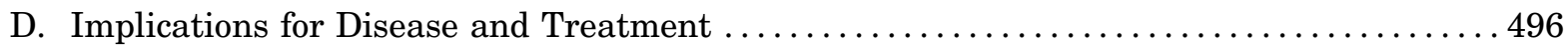

1. Pressurized Resistance Vessels, Implications of the Myogenic Response for Hypertension, and Critical Analysis of Inhibitors. ..................... 496

2. Smooth Muscle Myosin versus Nonmuscle Myosin, Implications for Force Maintanance and Vascular Tone................................... 497

3. Force Maintenance/Latch and the Regulation of Vascular Tone: The Tonic versus Phasic Contractile Phenotype and Contributions to Pathogenesis of Hypertension. . . 499

4. Autoregulation of Vascular Resistance/Flow-Mediated Vasodilatation and Nitric Oxide Signaling with Analysis of Current Inhibitors. ................. 500

5. Mouse Models (Contractile Protein Knockout) and Implications for Hypertension. .... 500

E. Summary of Contractile Phenotype and Contributions to Pathogenesis of

Hypertension with Analysis of Current Therapies for Hypertension . . . . . . . . . . . . 502

F. Potential Novel Targets for Treatment of Essential Hypertension . . . . . . . . . . . . . 502

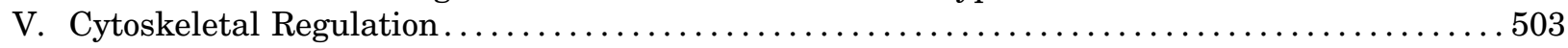

A. Intermediate Filaments, Dystrophin, Utrophin, and Microtubules .............. 503

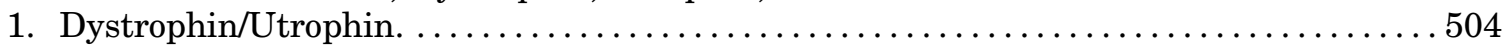

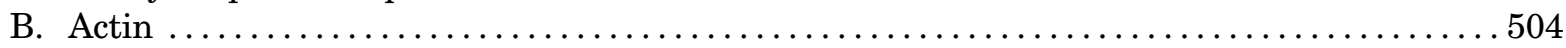

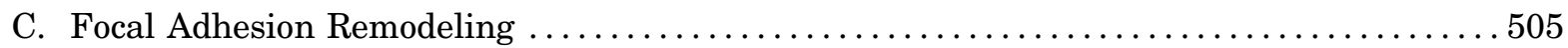

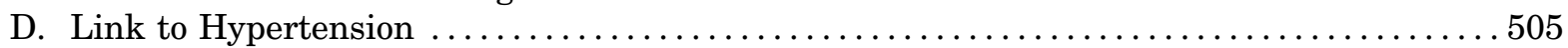

VI. Identifying Therapeutic Targets in Vascular Smooth Muscle through Biomechanical Studies 506

A. Arterial Stiffness as a Predictor of Negative Cardiovascular Events with Aging. ....... 506

1. Pulse Wave Velocity: The Clinical Standard ............................ 506

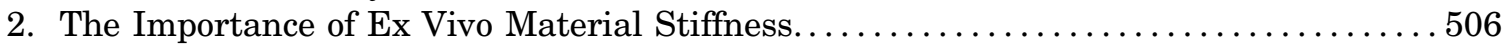

B. Regulation of Arterial Stiffness by Vascular Smooth Muscle .................... 507

1. Homeostatic Interactions between Cellular and Extracellular Components of the Arterial Wall.............................................. 507

2. The Focal Adhesion and Actin Cytoskeleton as Regulatory Sites of Arterial Material Stiffness......................................... 507

VII. Regulation of Vascular Smooth Muscle Cell Function by Epigenetic Mechanisms .......... 508

A. DNA Methylation ............................................... 509

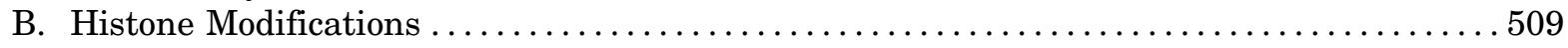

1. Histone Acetylases and Histone Deacetylases.......................... 510

a. Histone deacetylases and link to hypertension....................... 510

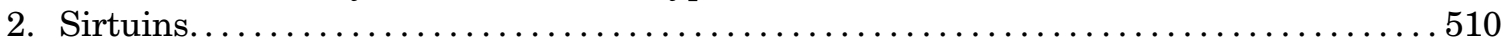

\begin{abstract}
ABBREVIATIONS: ACE, angiotensin converting enzyme; AM, rigor state; Ang II, angiotensin II; ARB, angiotensin receptor blocker; AT1, angiotensin type 1 ; $\mathrm{BK}, \mathrm{Ca}^{2+}$-dependent $\mathrm{K}^{+}$channels; $\mathrm{BP}$, blood pressure; $\mathrm{CaCC}, \mathrm{Ca}^{2+}$ gated $\mathrm{Cl}^{-}$channel; $\mathrm{CaD}$, caldesmon; $\mathrm{Ca}$, extracellular $\mathrm{Ca}^{2+}$; CaP, calponin; CCB, calcium channel blocker; CCt, C-terminal end of the LTCC; CI, central insert; $\mathrm{CICR} \mathrm{Ca}^{2+}$-induced Ca ${ }^{2+}$ release; $\mathrm{CO}$, cardiac output; CRAC, calcium release activated calcium channel; CREB, cAMP response element-binding protein; CVD, cardiovascular disease; EC, endothelial cells; ECM, extracellular matrix; ELC17, 17-kDa essential myosin light chain; eNOS, endothelial nitric oxide synthase; ERK, extracellular regulated kinase; FA, focal adhesion; FAK, focal adhesion kinase; GAP, GTPase activating protein; GEF, guanine nucleotide exchange factor; GWAS, genome wide-association studies; HAT, histone acetylase; HDAC, histone deacetylase; HF, heart failure; IL, interleukin; KLF, Kruppel-like factor; KO, knockout; LTCC, L-type $\mathrm{Ca}^{2+}$ channels; LncRNA, long noncoding RNA; LZ, leucine zipper; MHC; muscle myosin heavy chain; miR, microRNAs; MLCK, myosin light chain kinase; MP, myosin phosphatase; NAD, nicotinamide adenine dinucleotide; NM, nonmuscle; NO, nitric oxide; PAH, pulmonary arterial hypertension; PASMC, pulmonary artery smooth muscle cells; PKGI, protein kinase G; pre-miRNA, preliminary miRNA; PWV, pulse wave velocity; RISC, RNA-induced silencing complex; RLC, regulatory myosin light chain; RyR, ryanodine receptors; SHR, spontaneously hypertensive rat; SIRT, sirtuin; SM, smooth muscle; SMA, slow isoform of smooth muscle myosin heavy chain; SMB, fast isoform of smooth muscle myosin heavy chain; SNP, single nucleotide polymorphism; SOCE, store-operated calcium entry; SR, sarcoplasmic reticulum; STIM, stromal interaction molecule; SVR, systemic vascular resistance; TNF, tumor necrosis factor; $3^{\prime}$ UTR, 3' untranslated; VSMC, vascular smooth muscle cells; WT, wild type.
\end{abstract}




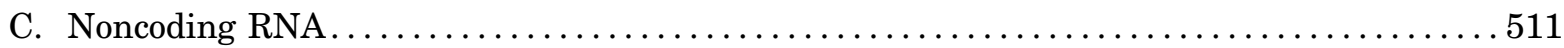

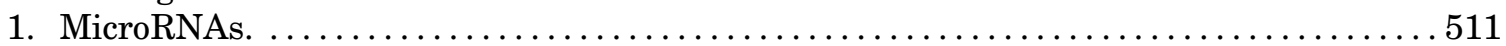

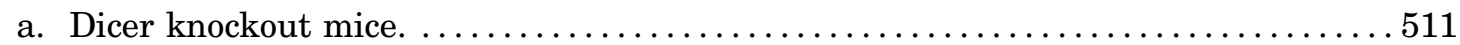

b. Regulation of vascular smooth muscle cell contractility. ................. 513

c. Regulation of vascular smooth muscle cell ion channels.................. 513

d. Regulation of the extracellular regulated kinase pathway . . . . . . . . . . . . 513

2. Long Noncoding RNAs. ......................................... 513

3. Strategies to Regulate microRNAs in Vascular Disease...................... 514

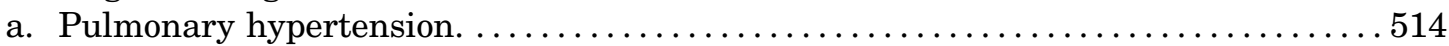

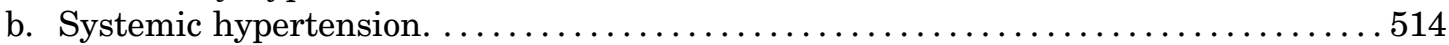

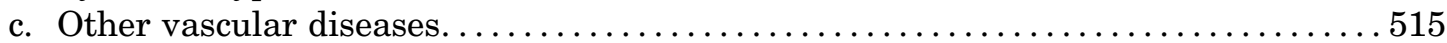

VIII. Vascular Smooth Muscle Diseases and Their Treatments ....................... 515

A. Review of Current Therapies and Their Targets $\ldots \ldots \ldots \ldots \ldots \ldots \ldots \ldots \ldots \ldots \ldots \ldots \ldots$

B. Other Major Vascular Diseases Including Analysis of Current Therapies

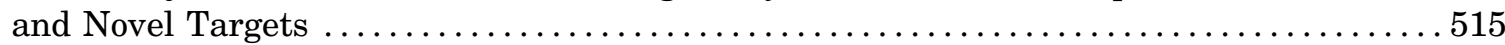

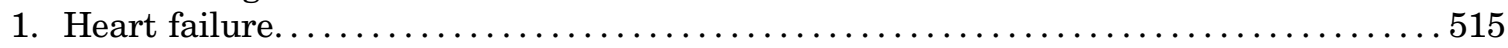

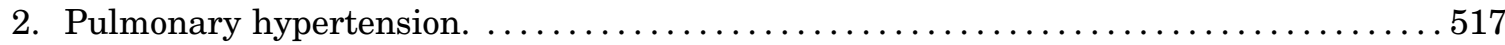

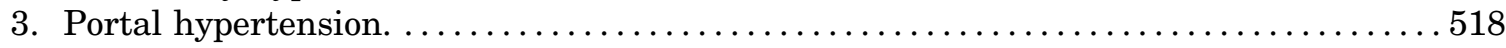

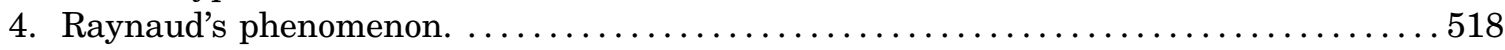

5. Pre-eclampsia/pregnancy-induced hypertension. $\ldots \ldots \ldots \ldots \ldots \ldots \ldots \ldots \ldots \ldots \ldots \ldots$

C. Personalized Medicine............................................ 519

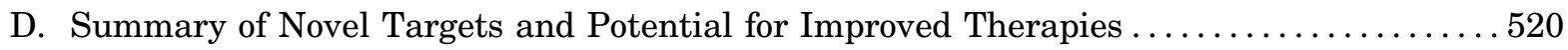

References.................................................... 520

\begin{abstract}
The smooth muscle cell directly drives the contraction of the vascular wall and hence regulates the size of the blood vessel lumen. We review here the current understanding of the molecular mechanisms by which agonists, therapeutics, and diseases regulate contractility of the vascular smooth muscle
\end{abstract}

\section{Introduction}

\section{A. Scope and Limitations}

The smooth muscle cells of blood vessels display considerable plasticity in their phenotype. In healthy, young blood vessels, the phenotype is largely contractile and blood pressure is well autoregulated. However, during the life span of an individual, vascular cells can switch to a synthetic, largely noncontractile phenotype in response to proinflammatory stimuli, diet or other factors that result in the development of atherosclerosis or vessel remodeling. We will not focus on these processes here but refer the reader to several recent reviews on this topic (Heusch et al., 2014; Brown and Griendling, 2015; Tabas et al., 2015).

Here we will focus on the contractile phenotype, which also can display plasticity of function through a range of more subtle adaptations to aging, biomechanical stress, and vasoactive physiologic and pathophysiologic molecules. The current review will focus on these responses and especially focus, as a prototype disease of contractile vascular smooth muscle, on the complex role of this cell type in hypertension and where many opportunities exist for the exploration of untapped potential therapeutic targets. cell and we place this within the context of whole body function. We also discuss the implications for personalized medicine and highlight specific potential target molecules that may provide opportunities for the future development of new therapeutics to regulate vascular function.

\section{B. Overview of Regulation of Blood Pressure/ Vascular Tone}

1. Guyton View of Regulation Blood Pressure, Kidney Role, Volume Regulation. In humans, the diagnosis of hypertension is widespread, but typically asymptomatic; $20-50 \%$ of the world's population has hypertension and in the United States $\sim 30 \%$ of the population is hypertensive (Hajjar et al., 2006). Furthermore, hypertension is a major risk factor for cardiovascular disease, stroke, and end-stage renal disease, and thus, there is significant morbidity and mortality associated with this disease. Because blood pressure (BP) is related to the cardiac output $(\mathrm{CO})$ and systemic vascular resistance $(\mathrm{SVR})$ by the equation $\mathrm{BP}=\mathrm{CO} \times \mathrm{SVR}$, increases in either CO or SVR should produce hypertension. Thus, although the molecular mechanism(s) that produce hypertension would be expected to be relatively straightforward, over 50 years of investigation have not defined the molecular mechanism(s) that underlies this medical condition.

The control of blood pressure is an integrated response that includes regulation by neural receptors, hormones, and renal fluid balance (Guyton, 1991). However, the handling of sodium within the kidney is well accepted to be the major factor that regulates blood 
pressure (Fig. 1), and hence, in the pathogenesis of hypertension renal $\mathrm{Na}^{+}$excretion, which regulates intravascular volume, is the primary determinant of cardiac output $(\mathrm{CO})$ and therefore blood pressure (Guyton, 1991). The role of control of intravascular volume by the kidney for the pathogenesis of hypertension is supported by the results of an elegant series of studies by Lifton's group (reviewed in Lifton et al., 2001). These investigators demonstrated that in humans, rare genetic causes of hypertension all arise from a defect in the handling of $\mathrm{Na}^{+}$in the kidney; mutations that increase $\mathrm{Na}^{+}$reabsorption (volume expansion) result in severe hypertension, whereas mutations that decrease $\mathrm{Na}^{+}$resorption (volume contraction) produce hypotension. We will not discuss the well-accepted role of renal fluid balance in regulation blood pressure, because this topic has been the subject of a number of reviews (Lifton et al., 2001; Oparil et al., 2003; Coffman and Crowley, 2008; Johnson et al., 2008).

2. Recent Direct Confirmation of Changes in Vascular Tone/Resistance Related to Changes in Systemic Vascular Resistance and Blood Pressure and the Importance of Vascular Smooth Muscle Contraction in both Normal Physiology and Pathophysiology-Hypertension. More than $90 \%$ of patients are diagnosed with essential hypertension, or hypertension of unknown etiology (Oparil et al., 2003). Fortunately, despite the lack of a clear mechanism, there are a number of classes of antihypertensive agents that effectively lower blood pressure. Intuitively, one would expect that changes in vascular tone would result in changes in systemic vascular resistance (SVR) and result in either hyperand/or hypotension. And, although a number of the classes of antihypertensive agents target the vascular smooth muscle $[\alpha$-blockers, angiotensin converting enzyme (ACE) inhibitors, angiotensin receptor blockers

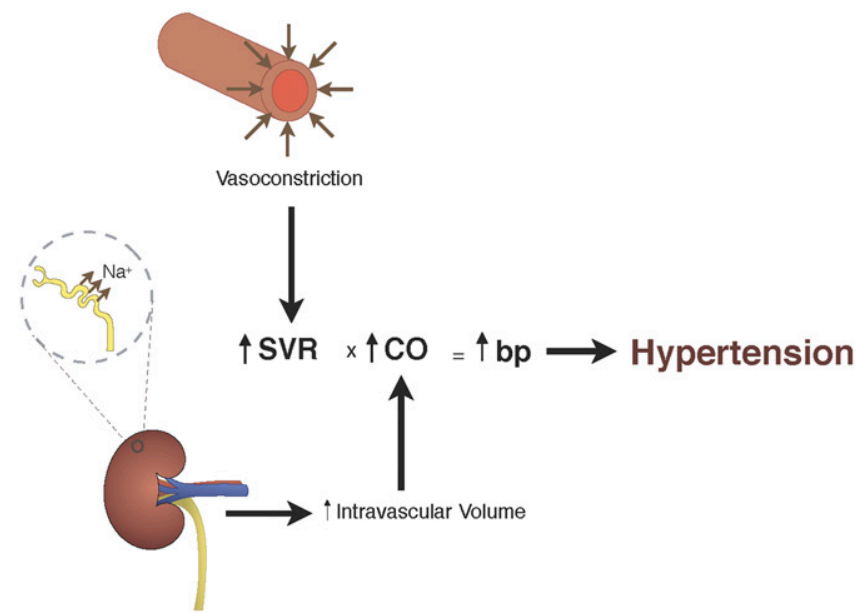

Fig. 1. SVR versus kidney: Blood pressure is the product of systemic vascular resistance and cardiac output $(\mathrm{BP}=\mathrm{SVR} \times \mathrm{CO})$. Changes in $\mathrm{Na}^{+}$ reabsorption will increase or decrease intravascular volume and result in an increase or decrease cardiac output, which will alter blood pressure. Similarly, alterations in vascular tone can either increase or decrease SVR, which leads to an increase or decrease in blood pressure (see text for details).
(ARBs), calcium channel blockers (CCBs)], until recently, there was little experimental evidence consistent with the regulation of vascular tone being an important factor for the molecular mechanism that produces hypertension (Fig. 1). However, a number of studies have demonstrated the importance of changes in vascular reactivity or the regulation of vascular smooth muscle contraction and/or vascular tone for the control of blood pressure. For these experiments, investigators have genetically modified a mouse to produce abnormalities in the regulation of vascular tone and/or vascular dysfunction; these mouse models include the $\mathrm{BKCa}^{2+}$ channel $\beta 1$ subunit knockout (KO) (Brenner et al., 2000), estrogen receptor $\beta \mathrm{KO}$ (Zhu et al., 2002), vascular smooth muscle cell Sur2 K(ATP) channel KO (Chutkow et al., 2002), endothelial nitric oxide synthase (eNOS) KO (Huang et al., 1995), RGS2 KO (Tang et al., 2003), PKGI KO (Tang et al., 2003), PKGI $\alpha$ leucine zipper mutant (Michael et al., 2008), and the MYPT1 KO (Qiao et al., 2014). All of these mice have both vascular dysfunction and hypertension, and these data suggest that vascular dysfunction produces hypertension. However, in these transgenic models, vascular dysfunction within the kidney could alter fluid balance and a resulting increase in intravascular volume and the resulting increase in $\mathrm{CO}$ could be responsible for producing hypertension. The most compelling argument that isolated vascular dysfunction results in hypertension are the results of Crowley et al. (2005). These investigators demonstrated that mice with a KO of the angiotensin type 1 (AT1) receptor were hypotensive. Furthermore, these investigators produced mice with the KO of the AT1 receptors in the kidney with normal AT1 expression in the peripheral vasculature, as well as the KO of AT1 receptors in the peripheral vascular smooth muscle with normal AT1 expression in the kidney. The blood pressure in these two strains was equal and intermediate between the AT1 KO and wildtype (WT) mice. These results demonstrate that in isolation, an abnormality in the regulation of vascular smooth muscle contraction produces a change in blood pressure, and therefore, an isolated increase in vascular smooth muscle tone will produce hypertension. Thus the regulation of vascular smooth muscle contraction is important in both health and disease.

3. Racial Differences/Personalized Medicine. Further complicating investigation of the mechanism underlying the pathogenesis of hypertension are racial differences in the effectiveness of the various classes of antihypertensives (Cushman et al., 2000; Johnson, 2008; Gupta, 2010), including the response to $\beta$-blockers, ACE inhibitors, and ARBs. White compared with black patients with hypertension are more likely to respond to $\beta$-blockers, ACE inhibitors, and ARBs, whereas for black patients, treatment with a diuretic or calcium channel blocker (CCB) is more likely to be effective (Johnson et al., 2008). Additionally, there also 
appears to be regional differences in the response to antihypertensive agents; there is a 10 state region in the Southeastern U.S., referred to as the Stroke Belt, in which the mortality from cerebral vascular accidents is $10 \%$ greater than the rest of the country. In this region, compared with the rest of the U.S., treatment of hypertension with diuretics, $\beta$-blockers, ACE inhibitors, and clonidine is less effective, whereas there is no difference in the effectiveness of CCBs and prazosin (Cushman et al., 2000). After controlling for race, the differences in the therapeutic success of diuretics and clonidine is still present. Furthermore, for black patients with hypertension in this region, similar to the rest of the U.S., CCBs are more likely to control blood pressure and the effectiveness of $\beta$-blockers and prazosin therapy is poor.

These racial differences in response to therapy are also present for the treatment of heart failure. Analysis of the results of the V-HeFT (Vasodilator-Heart Failure) trial demonstrated that treatment of black patients with heart failure with the combination of hydralazine and isosorbide dinitrate reduced mortality, whereas this regimen did not change mortality compared with placebo for white patients (Carson et al., 1999). In contrast to these results, treatment of heart failure with enalapril reduced mortality in white, but not black, patients, and in white patients, enalapril produced a larger reduction in blood pressure and regression of cardiac size than hydralazine and isosorbide dinitrate (Carson et al., 1999).

These racial and regional differences in the response to antihypertensive regimens could be due to polymorphisms. A number of genome wide-association studies (GWAS) have investigated this question (reviewed in Cushman et al., 2000; Johnson et al., 2008), and these studies as well as their implications will be discussed later in this review. However, changes in the vascular smooth muscle phenotype could be responsible for diversity in the effectiveness of the different classes of antihypertensive agents. Defining the role of the vascular smooth muscle phenotype in the pathogenesis of hypertension could identify novel therapeutic targets, which could be exploited in rational drug design. Furthermore, comparing the vascular smooth muscle phenotype between races and regions could potentially define the mechanism that governs the heterogeneity in the response to antihypertensive therapy and form the basis for an individualized approach for selecting an effective antihypertensive regimen.

\section{Regulation of $\mathbf{C a}^{2+}$}

\section{A. $\mathrm{Ca}^{2+}$ Determines Vascular Smooth Muscle Cell Contractility and Phenotype}

Vascular smooth muscle cells (VSMC), like all other muscle cells, depend on $\mathrm{Ca}^{2+}$ influx to initiate contraction. However, the VSMC intracellular $\mathrm{Ca}^{2+}$ concentration does not only determine the contractile state, but also affects the activity of several $\mathrm{Ca}^{2+}$ dependent transcription factors and thereby determines VSMC phenotype. To govern the various $\mathrm{Ca}^{2+}$-dependent functions and in reaction to different stimuli, VSMCs use a variety of plasmalemmal and sarcoplasmic reticulum (SR) $\mathrm{Ca}^{2+}$ channels to produce a large repertoire of $\mathrm{Ca}^{2+}$ signals, which differ in their spatial and temporal distribution (reviewed by Amberg and Navedo, 2013). These signals range from cell-wide changes in $\left[\mathrm{Ca}^{2+}\right]$ to highly localized $\mathrm{Ca}^{2+}$ entry or release events. $\mathrm{Ca}^{2+}$ can enter the cell from the extracellular space or be released from the largest intracellular $\mathrm{Ca}^{2+}$ store, the sarcoplasmic reticulum (SR). Extracellular $\mathrm{Ca}^{2+}$ influx is mainly mediated by the opening of voltage dependent L-type $\mathrm{Ca}^{2+}$ channels (LTCC), but there are a number of other channels that modulate intracellular $\mathrm{Ca}^{2+}$, including transient receptor potential (TRP) cation channels. Because of their high single-channel conductance and expression in VSMCs, LTCCs have the largest influence on global $\left[\mathrm{Ca}^{2+}\right]_{i}$, and their activity largely determines the VSMC's contractile state and ultimately vessel diameter (Knot and Nelson, 1998). In response to agonist activation of SR-bound inositol trisphosphate (IP3) or ryanodine receptors (RyR), $\mathrm{Ca}^{2+}$ is released into the cytoplasm from the SR. Local $\mathrm{Ca}^{2+}$ signals from the plasmalemma or the junctional SR can augment or oppose increases in global $\mathrm{Ca}^{2+}$ through the activation of $\mathrm{Ca}^{2+}$-dependent ion channels and their regulatory signaling molecules that ultimately affect plasma membrane potential and therefore LTCC activity.

\section{B. Compartmentalization of $\mathrm{Ca}^{2+}$ Signaling}

The concept of $\mathrm{Ca}^{2+}$ compartmentalization was introduced when it was demonstrated that local increases in $\mathrm{Ca}^{2+}$ could activate the contractile apparatus without influencing other $\mathrm{Ca}^{2+}$-dependent signaling pathways (Karaki, 1989). $\mathrm{Ca}^{2+}$ is slow to diffuse across the cytoplasm (Berridge, 2006) and a large flux of $\mathrm{Ca}^{2+}$ is required to achieve the high $\mathrm{Ca}^{2+}$ concentration necessary for activation of $\mathrm{Ca}^{2+}$-dependent processes. Therefore, to compartmentalize and regulate $\mathrm{Ca}^{2+}$ signals, VSMCs arrange their organelles in a fashion that limits the space for diffusion and thereby increases the effect of local changes in $\left[\mathrm{Ca}^{2+}\right.$ (Kargacin, 1994; Poburko et al., 2004) (Fig. 2). The effects of $\mathrm{Ca}^{2+}$ entry hence depend on the way that organelles, $\mathrm{Ca}^{2+}$ pumps, channels, and $\mathrm{Ca}^{2+}$-dependent signaling molecules are organized in signaling microdomains around the source of the $\mathrm{Ca}^{2+}$ signal, as well as its duration and amplitude. More on the organization of such microdomains in VSMCs and how they affect VSMC contractility and phenotype can be found in the review on regulation of cellular communication by signaling microdomains by Billaud et al. (2014).

1. $\mathrm{Ca}^{2+}$ Sparklets. Local increases in cytoplasmic $\mathrm{Ca}^{2+}$ resulting from influx through single or small 


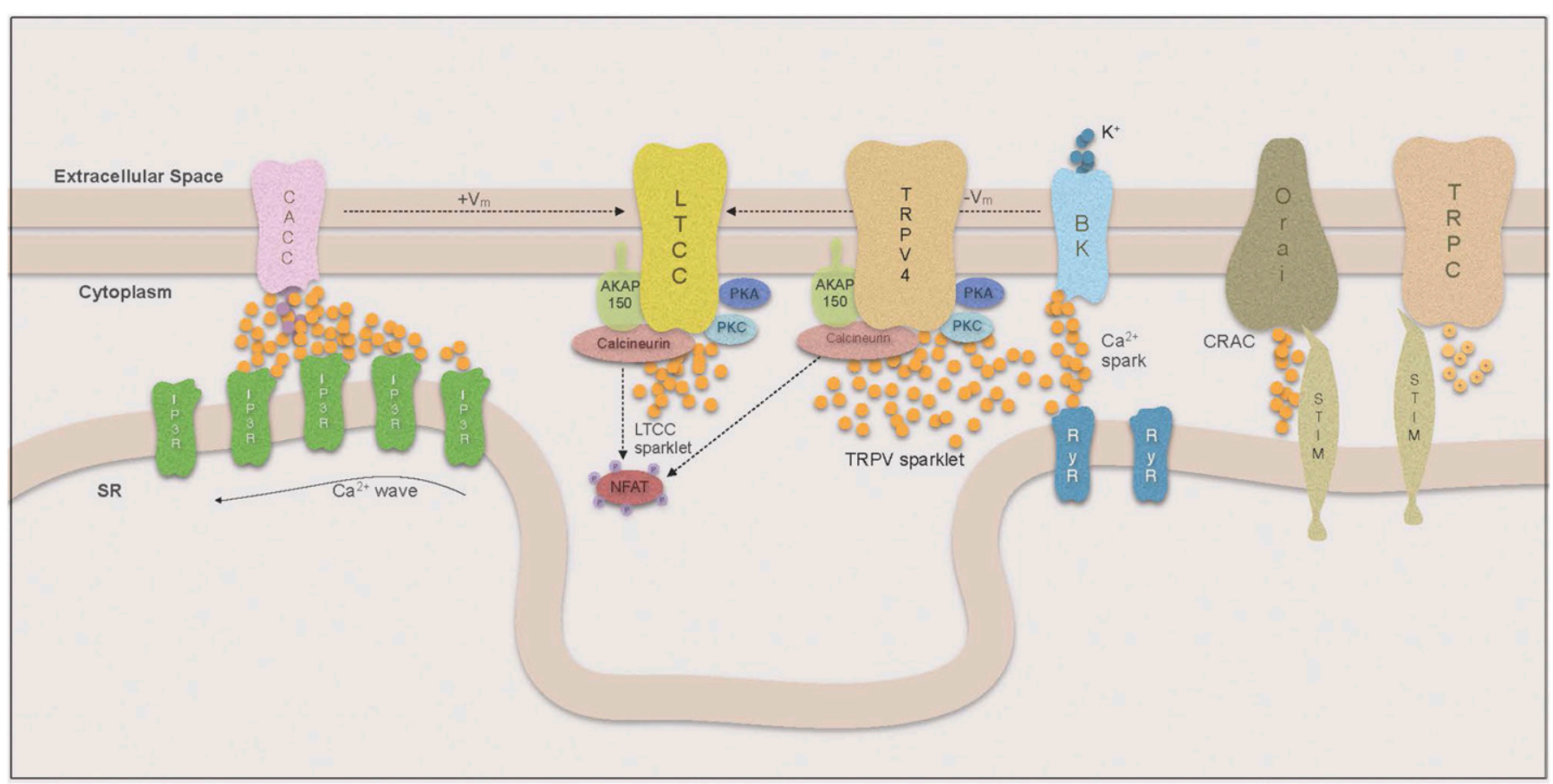

Fig. 2. Compartmentalization of Ca signaling.

clusters of LTCCs are called $\mathrm{Ca}^{2+}$ sparklets (reviewed by Navedo and Amberg, 2013). Because of the steep voltage sensitivity of LTCCs, the sparklet frequency and persistence are closely linked to membrane potential. Thus local changes in membrane potential will result in alterations of local sparklet activity, whereas cell-wide depolarization leads to extensive opening of LTCCs and global influx of $\mathrm{Ca}^{2+}$ (Navedo et al., 2005; Amberg et al., 2007). Increases in $\left[\mathrm{Ca}^{2+}\right]_{\mathrm{i}}$ and $\mathrm{Ca}^{2+}$ sensitivity of the contractile apparatus in VSMCs are considered hallmarks of essential hypertension, and it has been widely assumed that the increase in intracellular $\mathrm{Ca}^{2+}$ is mediated by increased influx through LTCCs. Consistent with this are results in the rat where banding was used to produce a sudden high intravascular pressure in the right renal artery. After only 2 days, VSMCs from the right renal artery showed increased expression of $\alpha_{1 \mathrm{C}}$ subunits of the LTCC and increased $\mathrm{Ca}^{2+}$ currents compared with VSMCs from the left renal artery (Pesic et al., 2004). However, the ratio of right renal artery/left renal artery $\alpha_{1 \mathrm{C}}$ subunit expression decreased over time, which may indicate a dynamic adjustment to this sudden pressure overload occurring within the VSMCs.

Surprisingly, overall LTCC expression and cell-wide $\mathrm{Ca}^{2+}$ influx was recently found to be decreased in a mouse model of essential hypertension (Tajada et al., 2013). However, although there was a decrease in the number of LTCCs present on the plasma membrane, the LTCCs showed increased local sparklet activity. These investigators demonstrated that fewer, but highly active, LTCCs were able to increase $\left[\mathrm{Ca}^{2+}\right]_{\mathrm{i}}$ locally as well as cell-wide. The activity of $\mathrm{Ca}^{2+}$ sparklets has been shown to depend on whether the LTCC is part of a pentad complex bound to the plasma membrane by the scaffolding protein AKAP150 (Navedo et al., 2008). LTCCs that are not coupled in such complexes have a higher probability of producing stochastic sparklets with low flux and short duration, whereas AKAPassociated channels can produce high-activity persistent sparklets.

The dynamics of these persistent sparklets are regulated by kinases and phosphatases that are targeted to a subpopulation of LTCCs by the plasmalemmal anchor AKAP150. Under physiologic conditions in these signaling microdomains, the formation of persistent sparklets mainly relies on protein kinase $\mathrm{C}$ (PKC) activity and is counteracted by the serine phosphatase calcineurin. In pathologic conditions such as diabetes, however, protein kinase $\mathrm{A}$ (PKA) becomes a mediator of enhanced sparklet activity (Navedo et al., 2010). In a study by Navedo et al. (2008) it was shown that the inhibition of cytoplasmic calcineurin with cyclosporine $\mathrm{A}$ in $\mathrm{AKAP}^{-/-}$ mice had no effect on LTCC sparklet activity, whereas the inhibition of AKAP150-anchored calcineurin in wildtype mice yielded an increase in persistent sparklets. This confirmed the hypothesis that there was a negative relationship between calcineurin and LTCC sparklet activity but highlighted the importance of calcineurin being targeted to the plasmalemma by AKAP150. The relevance of PKC interaction with LTCCs in the development of ATII-induced hypertension has been demonstrated in a number of experiments, in which not only the $\mathrm{KO}$ of PKC but also the ablation of AKAP150 lead to an 
inability of ATII infusion to produce hypertension (Navedo et al., 2008). In this model, the level of cellular PKC was unchanged in AKAP150 ${ }^{-/-}$VSMCs. These data suggest that recruitment of PKC to the LTCC by AKAP150 is crucial for the development of this form of hypertension. AKAP150 is also thought to play a role in the functional coupling of LTCCs to each other, which amplifies $\mathrm{Ca}^{2+}$ influx and is, similar to persistent sparklet activity, increased in hypertension (Nieves-Cintron et al., 2008). Although the mechanism of coupled gating is still under investigation, a model has been proposed by which coupled gating is mediated by calmodulin (CaM)dependent interactions between the carboxy-terminals of AKAP150-coupled LTCCs and is increased with PKC activation and calcineurin inhibition (Navedo et al., 2010; Cheng et al., 2011).

It should be noted, that in some studies, there was significant PKC activation in agonist-mediated vasoconstriction, but not in pressure-mediated vasoconstriction (Jarajapu and Knot, 2005; Ito et al., 2007), suggesting that PKC has a negligible role in myogenic tone. However, other groups reported PKC involvement in the modulation of the arteriolar myogenic response to increased intravascular pressure (Hill et al., 1990). This study demonstrated that inhibition of PKC led to inhibition of the myogenic response, whereas a stimulator of PKC activity increased myogenic responsiveness.

In a recent study (Mercado et al., 2014), investigators demonstrated that AKAP150-recruited PKC also regulates the activity of $\mathrm{Ca}^{2+}$-permeable, nonselective TRPV4 channels. These channels can produce $\mathrm{Ca}^{2+}$ sparklets with 100 -fold higher $\mathrm{Ca}^{2+}$ flux compared with LTCCs, yet they have been linked to VSMC relaxation (Earley et al., 2009). This association results from the high $\mathrm{Ca}^{2+}$ flux that enables TRPV4 to stimulate SRmembrane bound RyRs in relative proximity to the plasmalemma as a form of $\mathrm{Ca}^{2+}$-induced $\mathrm{Ca}^{2+}$ release (CICR) found in VSMCs.

In contrast to TRPV4 sparklets, LTCC sparklet flux is much lower and therefore not sufficient to trigger $\mathrm{Ca}^{2+}$ release from the SR, but overall LTCC sparklet activity is higher and hence LTCCs have a much greater effect on global $\mathrm{Ca}^{2+}$. Through the effect on global $\mathrm{Ca}^{2+}$, LTCC sparklet activity determines the rate at which the SR can refill its $\mathrm{Ca}^{2+}$ stores. However, neither the $\mathrm{SR} \mathrm{Ca}^{2+}$ content nor the number and amplitude of SR $\mathrm{Ca}^{2+}$ release events appear to be directly linked to LTCC sparklet activity (Collier et al., 2000; Essin et al., 2007).

Other important members of the TRP channel family include TRPC1, TRPC3, TRPC6, and TRPM4. They have been found to have a role in regulating myogenic tone as well as the myogenic response and are known to be involved in the mechanism of action of vasoconstrictors (refer to reviews by Beech, 2005, 2013) and will be discussed in the section II.B.4. However, for details on the role of TRP channels in vascular function and how the dysregulation of vascular as well as endothelial TRP channels is related to vascular-related pathologies, please see the recent review by Earley and Brayden (2015).

2. $\mathrm{Ca}^{2+}$ Sparks. Highly restricted and large $\mathrm{Ca}^{2+}$ release events through SR RyRs are called $\mathrm{Ca}^{2+}$ sparks, and $\mathrm{Ca}^{2+}$ sparks have an important regulatory role in VSMCs. Similar to sparklets, their spatial reach is small, so they have no direct effect on contractility; however, the proximity of RyRs to the plasma membrane allows them to affect global $\left[\mathrm{Ca}^{2+}\right]_{\mathrm{i}}$ indirectly (reviewed by Amberg and Navedo, 2013). The nature of the VSMC's response to $\mathrm{Ca}^{2+}$ sparks depends on the $\mathrm{Ca}^{2+}$-activated plasmalemmal ion channels that are spatially coupled to the RyRs. In many VSMC, tissues sparks are targeted to large conductance $\mathrm{Ca}^{2+}$-dependent $\mathrm{K}^{+}$channels (BK) that oppose vasoconstriction by allowing hyperpolarizing outward $\mathrm{K}^{+}$currents (Nelson et al., 1995). On the other hand, $\mathrm{Ca}^{2+}$ gated $\mathrm{Cl}^{-}$channels (CaCCs) depolarize the plasmalemma and thereby enhance $\mathrm{Ca}^{2+}$ influx through LTCCs (Kitamura and Yamazaki, 2001; Leblanc et al., 2005).

a. $\mathrm{Ca}^{2+}$-dependent $\mathrm{K}^{+}$channel-coupled sparks. A single $\mathrm{Ca}^{2+}$ spark increases the open probability of about $30 \mathrm{BK}$ channels in its proximity by 100 -fold (Jaggar et al., 2000; Perez et al., 2001). Sparks can occur spontaneously or be triggered by TRPV4 sparklets in the form of a CICR mechanism. Structurally, plasmalemmal BK channels in VSMCs are formed by four pore forming alpha subunits encoded by the slo gene and regulatory $\beta 1$ subunits that are not necessary for the formation of a functional channel (Toro et al., 1998). However, the $\beta 1$ subunits play a significant role in modulating the $\mathrm{Ca}^{2+}$ sensitivity and hence functional coupling to RyRs (Brenner et al., 2000). It has been demonstrated by several groups that ablation of the $\beta 1$ subunit in mice leads to desensitization to $\mathrm{Ca}^{2+}$ and functional uncoupling of BK channels from $\mathrm{Ca}^{2+}$ sparks, causing membrane depolarization, increases in arterial tone, and eventually hypertension (Brenner et al., 2000; Pluger et al., 2000). Furthermore for ATII-induced hypertension, it has been reported that the $\beta 1$, but not the pore-forming alpha subunit, is downregulated, which mediates a decrease in the sensitivity of BK channels and thereby contributes to vascular dysfunction (Amberg et al., 2003; Nieves-Cintron et al., 2007). Consistent with these results, associations between gain of function mutations of the $\beta 1$ subunit and a lower prevalence of diastolic hypertension have been described (Fernandez-Fernandez et al., 2004; Nelson and Bonev, 2004; Senti et al., 2005). Additionally, it has been demonstrated that $\beta 1$ subunit downregulation in ATII-induced hypertension is mediated by enhanced activity of the transcription factor NFATc3 (Amberg et al., 2004; Nieves-Cintron et al., 2007). However in hypertensive animals, there have also been studies that have found higher expression of the $\alpha$ subunit in VSMCs, suggesting that the BK channel is primarily 
involved in a compensatory response to increased VSMC tone from enhanced LTCC or decreased $\mathrm{K}_{\mathrm{v}}$ activity (reviewed in Cox and Rusch, 2002). Hence BK channels appear to be involved in the pathogenesis in some as well as compensation and protection in other forms of hypertension.

Using different strategies to modulate plasmalemmal $\mathrm{K}^{+}$channel activity to inhibit $\beta 1$ downregulation in developing hypertension or to increase $\beta 1$ expression in VSMCs would appear to be a promising approach for the treatment of hypertension. In addition, a number of BK channel openers are currently in development (Webb et al., 2015); however, the use of BK channel openers for the treatment of hypertension is limited by concerns for off-site effects in other smooth muscle tissues. As the $\beta 1$ subunit of the BK channel seems to be expressed predominantly in VSMCs (Tanaka et al., 1997), targeting $\beta 1$ expression through gene therapy or modulation of the NFATc3 pathway represents a possible alternative (reviewed by Joseph et al., 2013).

b. $\mathrm{Ca}^{2+}$ gated $\mathrm{Cl}^{-}$channel-coupled sparks. In some VSMCs, $\mathrm{Ca}^{2+}$ sparks are coupled to $\mathrm{CaCCs}$, and their activation is followed by "spontaneous transient inward currents" or STICs. The two families of CaCCs that have only recently been identified are called bestrophins and TMEM16A. They are also expressed in renal tubular epithelium as well as the heart and are hence thought to have a multidimensional role in blood pressure regulation (reviewed by Matchkov et al., 2015). Because the activation of these channels results in plasma membrane depolarizing currents, they are thought to have an amplifying effect on vascular contractile stimuli by indirectly causing the opening of LTCCs (Leblanc et al., 2005; Matchkov et al., 2013; Bulley and Jaggar, 2014). Indeed, downregulation or inhibition of TMEM16A led to decreased arterial constriction in a variety of studies (Jensen and Skott, 1996; Bulley et al., 2012; Davis et al., 2013; Dam et al., 2014), and a smooth muscle KO of TMEM16A in mice lead to a decrease in the ability of ATII infusion to produce hypertension. As CaCCs are also permeable to other anions such as $\mathrm{HCO}_{3}{ }^{-}$, it is also possible that some effect may be due to changes in intracellular $\mathrm{pH}$ that would affect $\mathrm{pH}$-sensitive enzymes including Rho kinase (Boedtkjer et al., 2011). Although there are a number of substances that can inhibit CaCC activity in vitro, the unknown molecular identity of TMEM16A as well as its expression in various tissues would suggest that there is poor pharmacological specificity in vivo and there would be many off target effects (Greenwood and Leblanc, 2007; Boedtkjer et al., 2008).

3. $\mathrm{Ca}^{2+}$ Waves. Activation of $\mathrm{CaCCs}$ is also often mediated by $\mathrm{Ca}^{2+}$ waves, a $\mathrm{Ca}^{2+}$ signal in which subsequent openings of $\mathrm{IP}_{3} \mathrm{Rs}$ and in some tissues RyRs on the SR cause a wave of $\mathrm{Ca}^{2+}$ release events across the entire length of the VSMC, usually close to the plasma membrane (Iino et al., 1994; Hill-Eubanks et al., 2011;
Amberg and Navedo, 2013). Westcott and colleagues described the contrasting roles of RyRs and $\mathrm{IP}_{3} \mathrm{Rs}$ for the effects of $\mathrm{Ca}^{2+}$ waves in arterioles and upstream feed arteries. Although arteriolar $\mathrm{Ca}^{2+}$ waves are solely $\mathrm{IP}_{3} \mathrm{R}$ mediated and therefore not inhibited by ryanodine, RyR inhibitors decreased $\mathrm{Ca}^{2+}$ waves in feed arteries. In both tissues, $\mathrm{Ca}^{2+}$ waves were inhibited with phospholipase $\mathrm{C}$ (PLC) inhibitors and $\mathrm{IP}_{3} \mathrm{R}$ blockers, which led to a decrease in $\left[\mathrm{Ca}^{2+}{ }_{i}\right]$ and vasodilation. Therefore, $\mathrm{IP}_{3} \mathrm{Rs}$ contribute to $\mathrm{Ca}^{2+}$ waves in both tissues as part of a positive feedback loop for myogenic tone. In contrast, despite the inhibition of $\mathrm{Ca}^{2+}$ sparks and waves in feed arteries, inhibition of RyRs caused an increase in $\left[\mathrm{Ca}^{2+}{ }_{i}\right]$ and led to vasoconstriction. This was abolished in the presence of BK-channel blocker paxilline, which supports the hypothesis that RyRs, which are involved in $\mathrm{Ca}^{2+}$ waves, are also coupled to BK channels and part of a negative feedback regulation of myogenic tone (Lamont et al., 2003; Westcott and Jackson, 2011; Stewart et al., 2012; Westcott et al., 2012).

In arterioles, $\mathrm{Ca}^{2+}$ waves are initiated by $\mathrm{IP}_{3^{-}}$ dependent opening of an $\mathrm{IP}_{3} \mathrm{R}$ creating a $\mathrm{Ca}^{2+}$ "blip," a single $\mathrm{IP}_{3} \mathrm{R}$ opening (Bootman and Berridge, 1996), or a "puff," short $\mathrm{Ca}^{2+}$ release from a small cluster of $\mathrm{IP}_{3} \mathrm{Rs}$ that is biophysically different from a RyR-mediated spark (Bootman and Berridge, 1996; Thomas et al., 1998). Subsequently, clusters of $\mathrm{IP}_{3} \mathrm{Rs}$ open in response to the $\mathrm{Ca}^{2+}$ released by adjacent $\mathrm{IP}_{3} \mathrm{Rs}$ (CICR) and are inactivated as the $\left[\mathrm{Ca}^{2+}\right]$ rises further. The $\mathrm{IP}_{3} \mathrm{R}$ 's $\mathrm{Ca}^{2+}$-dependent activation/inactivation properties are reflected in the wave-like pattern of $\mathrm{IP}_{3} \mathrm{R}$-mediated $\mathrm{Ca}^{2+}$ release (Foskett et al., 2007). $\mathrm{Ca}^{2+}$ wave initiation depends on $\mathrm{IP}_{3}$ synthesis by PLC, which occurs after activation of G-protein-coupled receptors by their respective agonists, including norepinephrine and endothelin-1 (Lamont et al., 2003; Westcott and Jackson, 2011; Stewart et al., 2012; Westcott et al., 2012). However, $\mathrm{Ca}^{2+}$ waves are also seen in the absence of agonists and depend on the spontaneous basal production of $\mathrm{IP}_{3}$ by PLC, which varies in different vascular beds, and thus will affect the frequency of $\mathrm{Ca}^{2+}$ release through RyRs via CICR (Gordienko and Bolton, 2002). In arterioles, the wave leads to VSMC contraction by directly increasing $\left[\mathrm{Ca}^{2+}\right]_{\mathrm{I}}$, and this effect is amplified by the $\mathrm{Ca}^{2+}$-dependent opening of CaCCs in the plasma membrane that leads to membrane depolarization and increased $\mathrm{Ca}^{2+}$ influx through LTCCs.

4. Store-Operated Calcium Entry. When $\mathrm{SR} \mathrm{Ca}^{2+}$ stores are depleted after release through $\mathrm{IP}_{3} \mathrm{Rs}$, the SR $\mathrm{Ca}^{2+}$ sensor STIM (stromal interaction molecule) relocates to the SR-plasmalemmal junction and physically interacts with and activates the selective $\mathrm{Ca}^{2+}$ channel Orai [CRAC (calcium release activated calcium channel); reviewed by Trebak, 2012]. For VSMCs in the normal physiologic contractile phenotype, the expression of STIM/Orai is relatively low, but its expression is upregulated when the VSMC changes its phenotype to 
the proliferative state (Potier et al., 2009). In a rodent STIM/Orai knockdown model, nuclear factor activated T-cells (NFAT) translocation to the nucleus was decreased and VSMC proliferation in response to vascular injury was impaired (Aubart et al., 2009; Guo et al., 2009; Zhang et al., 2011). In spontaneously hypertensive rats, STIM/Orai is upregulated and depletion of SR stores lead to greater SOCE, which may represent an independent mechanism leading to increased VSMC $\left[\mathrm{Ca}^{2+}\right]_{\mathrm{i}}$ in hypertension (Giachini et al., 2009b). Furthermore, these investigators found evidence suggesting that increased STIM/Orai activity may underlie sex differences in the development of hypertension. They determined that inactivation of the STIM/Orai mechanism with CRAC inhibitors as well as antibodies to STIM or Orai during and after store depletion abolished sex differences in spontaneous contractions of VSMCs (Giachini et al., 2009a). Thus CRACs represent a novel target in the treatment of hypertension.

However, there are a number of studies also suggesting a role of TRPC channels in SOCE (reviewed by Beech, 2012). Both TRPCs and Orai channels can be activated by STIM after store depletion (Zeng et al., 2008; Park et al., 2009); however, their individual contribution to SOCE is variable. Studies have demonstrated a partial suppression of SOCE by Orai and TRPC siRNA, respectively ( $\mathrm{Li}$ et al., 2008). The nature of TRPC-Orai interaction, or if there is in fact one, is currently unresolved (refer to Earley and Brayden, 2015), but both channels can also be activated independently from store depletion or $\mathrm{Ca}^{2+}$ release and their downstream effects on activation differ. TRPs exhibit multiplicity of gating and hence have been suggested to integrate various cellular signals including store depletion (Albert and Large, 2002). TRPC1 mediates $\mathrm{Ca}^{2+}$ influx after store depletion with thapsigargin $(\mathrm{Xu}$ and Beech, 2001; Sweeney et al., 2002; Lin et al., 2004) and is thought to be involved in contractile modulation and regulation of cell proliferation; however, more data are needed to determine its exact function. TRPC6 is a channel that mediates cation movement in a variety of experimental settings. In some tissues, inhibition of TRPC6 leads to a decrease in SOCE, but it also appears to be involved in SRindependent signaling. Studies demonstrated that TRPC6 is store and receptor operated, as well as stretch and osmotically activated in VSMCs. It can associate and form heteromultimers with TRPC3, which leads to tonic channel activation (Dietrich et al., 2003). TRPC3 and TRPC6 are upregulated in idiopathic pulmonary hypertension and an siRNA-induced decrease of TRPC6 expression decreases proliferation of cultured pulmonary artery VSMC isolated from patients with pulmonary hypertension. Furthermore, chronic hypoxia increases TRPC6 expression, whereas the ET-1 antagonist bosentan, a common treatment of PAH, lowers TRPC6 expression in pulmonary VSMCs (Kunichika et al., 2004; Lin et al., 2004). These are merely examples of the various roles TRPC channels occupy in VSMC signaling and a complete discussion of TRPC channels in health and disease has been recently presented in a number of reviews (Beech, 2005, 2012; Earley and Brayden, 2015).

\section{Excitation-Transcription Coupling}

An important mode in which $\mathrm{Ca}^{2+}$ can regulate VSMC contractility is by regulating the composition of the contractile apparatus, ion channels, and cellular signaling molecules by influencing VSMC gene transcription (reviewed by Kudryavtseva et al., 2013). In certain cytoplasmic locations, high $\left[\mathrm{Ca}^{2+}\right]$ activates specific kinases or phosphatases that in turn lead to activation and translocation of transcription factors to the nucleus. In the nucleus, $\mathrm{Ca}^{2+}$ can bind helix-loop-helix-loop structural domain or motif (EF hand) containing transcription factors directly (Carrion et al., 1999) or modulate transcription factor binding via $\mathrm{Ca}^{2+} / \mathrm{CaM}-\mathrm{S} 100$ complexes (Hermann et al., 1998). Although in many disease states VSMCs can completely lose their contractile function due to a phenotype switch toward a proliferative ECM-producing phenotype, more subtle changes within the contractile phenotype are also thought to play a role in the increased VSMC contractility observed in hypertension.

NFAT is a major target of calcineurin, and it translocates to the nucleus upon calcineurin-mediated dephosphorylation. Calcineurin activation is enhanced by the activity of the AKAP150-bound LTCC signaling pentad (Oliveria et al., 2007; Nieves-Cintron et al., 2008). Hence, NFAT activity is regulated by the level of persistent sparklet activity, but is also dependent on simultaneous inhibition of its nuclear export (Gomez et al., 2003). Interestingly, although membranedepolarizing signals such as $\mathrm{IP}_{3} \mathrm{R}$-mediated $\mathrm{Ca}^{2+}$ waves are thought to cause an increase in NFATc3 activation via enhanced LTCC activity, RyR-mediated $\mathrm{Ca}^{2+}$ release from the SR decreases NFAT activity by an LTCC independent mechanism (Gomez et al., 2002). SOCE has also been implicated in NFAT activation, and its disruption led to reduced hypoxia-induced NFAT nuclear translocation in pulmonary VSMCs (Bierer et al., 2011; Hou et al., 2013). Although a variety of $\mathrm{Ca}^{2+}$ signals lead to NFAT activation, persistently raised levels of intracellular $\mathrm{Ca}^{2+}$ fail to induce NFAT (Stevenson et al., 2001; Gonzalez Bosc et al., 2004). Therefore it is thought that oscillating $\mathrm{Ca}^{2+}$ signals (Matchkov et al., 2012) and concomitant inhibition of nuclear export (Gomez et al., 2003) leads to nuclear NFAT accumulation. It is well documented that inhibition of the calcineurin/NFAT pathway reduces VSMC proliferation, neointima formation, and vascular remodeling in response to injury (Liu et al., 2005; Nilsson et al., 2008; Esteban et al., 2011; Hou et al., 2013). However there are also studies indicating a role 
for NFAT within the contractile phenotype, altering the expression of plasmalemmal ion channels including BK (Nieves-Cintron et al., 2007) and $\mathrm{K}_{\mathrm{v}}$ channels (Amberg et al., 2004) and thereby increasing VSMC contractility and ultimately arterial tone.

In contrast to NFAT, CREB is regulated by the $\mathrm{Ca}^{2+}$ dependent kinases CaMKII and CaMKIV (Cartin et al., 2000). $\mathrm{Ca}^{2+}$ influx through LTCCs is important for activated phospho-CREB to accumulate in the nucleus (Stevenson et al., 2001). Signals that increase LTCC activity including $\mathrm{IP}_{3} \mathrm{R}$-mediated $\mathrm{Ca}^{2+}$ waves (Barlow et al., 2006) and SOCE (Pulver et al., 2004; Takahashi et al., 2007) lead to increased CREB-induced transcription, whereas $\mathrm{Ca}^{2+}$ sparks counteract CREB activity by hyperpolarizing the plasmalemma and reducing LTCC flux (Cartin et al., 2000; Wellman and Nelson, 2003). Because CREB activates genes involved in the contractile, as well as the proliferative phenotype, the ultimate effect of CREB activation on VSMC phenotype has not yet been determined. However in contrast to NFAT, CREB is induced by any signal that causes a sustained increase in $\mathrm{Ca}^{2+}$ entry through LTCCs.

In addition to controlling transcription indirectly through CREB, LTCCs have also been found to directly influence gene expression in VSMCs. In a study by Bannister et al. (2013) it was determined that when the C-terminal end of the LTCC (CCt) is cleaved, it either reassociates with LTCCs and reduces LTCC sparklet activity or it relocates to the nucleus and inhibits the transcription of LTCCs. The CCt thus acts as a bimodal vasodilator by decreasing LTCC transcription and reducing voltage-dependent LTCC opening. However, the enzyme responsible for CCt cleavage and the mechanism (s) for regulation have yet to be determined. Potentiating the effects of CCt through increased cleavage or possibly stimulation of CCt promoter sequences may offer another novel approach to controlling vascular contractility.

\section{Conclusion}

There are a variety of $\mathrm{Ca}^{2+}$-mediated mechanisms that increase VSMC contractility and are possible targets in antihypertensive therapy, some of which are well understood and can be specifically inhibited in vitro. However the development of novel treatments is often limited by the expression of the targets in nonvascular smooth muscle tissues and thus the many off site effects. A possible solution to this issue could be targeting specific therapies to VSMCs using viral vectors. There are a number of successful proof of concept studies using this technique that were recently reviewed by Joseph et al. (2013). Another issue that limits progress in the effort to find novel pharmacologic therapies in $\mathrm{Ca}^{2+}$ signaling is that the composition of $\mathrm{Ca}^{2+}$ signaling microdomains differs in various vascular beds, and hence results cannot always be generalized for VSMCs. This problem again highlights the importance of genetic $\mathrm{KO}$ and knockdown studies as a tool to explore targets for gene therapy for the treatment of hypertension.

\section{Vascular Smooth Muscle Signal Transduction}

\section{A. Signaling Pathways-Overview}

Many potential therapeutic strategies are designed to activate or inhibit specific signaling pathways in the vascular smooth muscle cell. It is clear that multiple vascular signaling pathways coexist as spatially separate signaling compartments in individual differentiated vascular smooth muscle (dVSM) cells and coordinated by a multitude of scaffolding proteins. However, these pathways are often overlapping, multilayered, and tissue specific. The tissue-specific nature of these pathways, even between different vessels or sizes of vessels, has led to much controversy on the relative importance of one pathway versus another. Ultimately, however, the possibility of multiple pathways that could be activated or inhibited in various disease states or as functional compensation to physiologic stress gives the system considerable functional plasticity.

At the simplest level, it is well established that vascular tone can be increased either by increasing activation of myosin (Pathways \#1 \& \#2, Fig. 3) or, in a manner analogous to that in striated muscle, by removal of inhibition of actin (Pathway \#3, Fig. 3). Either mechanism will lead to an increase in actomyosin activation and crossbridge cycling. Recently, several laboratories have reported more controversial mechanisms by which agonists or biomechanical forces can regulate both vascular and airway smooth muscle contractility by remodeling cytoskeletal attachments (Walsh and Cole, 2013; Zhang et al., 2015) (Pathway \#4, Fig. 3). These four pathways are discussed in more detail below.

1. Major Pathways Leading to Changes in the Activity of Smooth Muscle Myosin. This has been a very active area of investigation by vascular smooth muscle biologists and, as discussed below, has already identified many potential pharmacologic target molecules and in some cases led to possible drug candidates.

Smooth muscle myosin differs from skeletal and cardiac myosins in that it lacks intrinsic myosin ATPase activity in the pure state. Smooth muscle myosin requires a posttranslational modification, phosphorylation of Ser 19 of the $20-\mathrm{kDa}$ regulatory light chain to display enzymatic activity. This phosphorylation is caused by a dedicated Ser/Thr kinase, myosin light chain kinase (MLCK). (Ito and Hartshorne, 1990)

MLCK is a $\mathrm{Ca} / \mathrm{CaM}$-dependent kinase and is most simply activated by increases in cytoplasmic ionized $\mathrm{Ca}$ $\left(\left[\mathrm{Ca}^{2+}{ }_{\mathrm{i}}\right]\right)$ levels (Pathway \#1, Fig. 3) such as occurs with a large number of G-protein coupled receptor-mediated agonists, such as alpha agonists or by depolarization of the cell membrane by channel activity or experimentally by equimolar replacement of $\mathrm{NaCl}$ with $\mathrm{KCl}$ in 


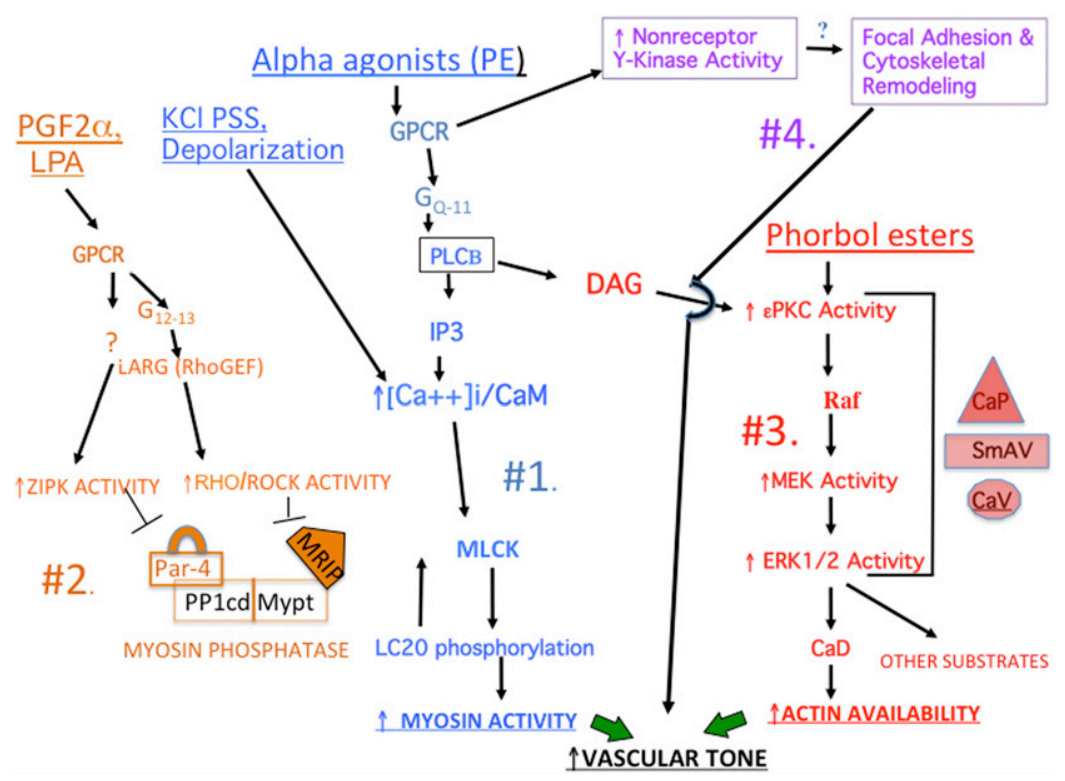

Fig. 3. Overview of pathways regulating vascular tone. See text for details. For additional detailed pathways, see subsequent figures.

physiologic saline solution. It has also been reported that increases in the free CaM level (Hulvershorn et al., 2001) or Ca-independent changes in the kinase activity of MLCK can also occur (Kim et al., 2000) by phosphorylation-mediated events.

Dephosphorylation of myosin by myosin phosphatase (MP) decreases its activity, and conversely, inhibition of MP will increase its activity. A large number of pathways, such as those activated by PGF2a and lysophosphatidic acid (Pathway \#2, Fig. 3), have been reported to inhibit MP through either Rho-associated protein kinase (ROCK)-dependent mechanisms or those involving Zipper-interacting protein kinase. These pathways are discussed in detail in section IV.

CaMKinase II is another $\mathrm{Ca} / \mathrm{CaM}$-dependent kinase with the interesting property, when activated, of autophosphorylating itself on T287, which leads to a sustained activity after $\mathrm{Ca}$ is removed, giving it a chemical "memory" of having been activated (Hudmon and Schulman, 2002; Lisman et al., 2002). Conversely, when S26 in the catalytic domain is autophosphorylated, it can terminate sustained kinase activity, making it "forget" prior activation (Yilmaz et al., 2013).

There are four main isoforms of CaMKII, the alpha, beta, gamma, and delta isoforms. The gamma (especially the G-2 variant) (Kim et al., 2000; Marganski et al., 2005) and delta (especially the $\mathrm{d} 2$ variant) (Ginnan et al., 2012) isoforms have been shown to play important roles in smooth muscle, with the gamma isoforms primarily regulating contractility and the delta isoforms regulating proliferation. To a large degree the gamma/delta ratio represents the degree of a phenotype switch between the contractile/proliferative phenotypes displayed by smooth muscle in different settings. Vascular injury reduces gamma isoform expression and upregulates delta expression (Singer,
2012). Conversely, siRNA-mediated knock down of the delta isoform attenuates VSM proliferation and neointimal formation. The conditional smooth muscle knockout of CaMKIIdelta significantly delays the progression of airway smooth muscle hyperresponsiveness to an ovalbumin challenge and this isoform is upregulated in the wild-type mouse in response to the same challenge. Thus the delta isoform may play a role in smooth muscle inflammatory responses (Spinelli et al., 2015).

With respect to the gamma isoform and its regulation of smooth muscle contractility, six smooth variants of the gamma isoform of CaMKII have been described with varying kinetics of $\mathrm{Ca} / \mathrm{CaM}$-dependent activation/deactivation (Gangopadhyay et al., 2003). One variant, the G-2 variant, which has unique sequence in the association domain of the kinase (Gangopadhyay et al., 2003), has been shown to have scaffolding properties with respect to ERK (extracellular regulated kinase). Antisense specific for the G-2 variant (Marganski et al., 2005) or generic against the gamma isoform or small molecule inhibitor studies (Kim et al., 2000; Rokolya and Singer, 2000) have all demonstrated roles for gamma CaMKII in the regulation of contractility. The CaMKII gamma G-2 variant is reported to be associated with vimentin intermediate filaments and dense bodies in unstimulated vascular smooth muscle cells and on activation by depolarization-mediated increases in cytosolic $\mathrm{Ca}^{2+}$ levels the G-2 variant translocates to the cortical focal adhesions (Marganski et al., 2005; Gangopadhyay et al., 2008). This variant also has been shown to be specifically dephosphorylated by an SCP3 phosphatase. SCP3 is a PP2C typed protein phosphatase, primarily expressed in vascular tissues and specifically binds to the unique association domain sequence in CaMKII gamma G-2. G-2 is bound to this phosphatase in 
unstimulated vascular smooth muscle tissue but is released upon depolarization-mediated $\mathrm{Ca}^{2+}$ influx. This phosphatase does not appear to regulate kinase activity but rather is thought to result in the exposure of a SH3 domain targeting of the kinase, which leads to targeting to focal adhesions (Gangopadhyay et al., 2008).

Although CaMKIIgamma is known to be activated by stimuli that increase the free $\mathrm{Ca}^{2+}$ level in dVSM, and antisense or inhibitors to CaMKII decrease the amplitude of the contraction to KCl PSS, the exact pathways that regulate contractility are still being confirmed. It has been shown that knock down of the gamma isoform or the G-2 variant specifically, as well as small molecule inhibitors of the kinase, lead to an inhibition of ERK activation and an inhibition of MLCK (Kim et al., 2000; Rokolya and Singer, 2000; Marganski et al., 2005). ERK has been shown to be capable of activating MLCK in other systems (Morrison et al., 1996; Nguyen et al., 1999), but whether this is the link in smooth muscle has not been definitively shown. Additionally in cultured vascular cells, CaMKII is rapidly activated after upon adherence of the cells upon plating onto ECM or polylysine. Adherence led to CaMKII-dependent tyrosine phosphorylation of paxillin and ERK activation ( $\mathrm{Lu}$ et al., 2005). The CaMKII delta 2 variant has also been shown to regulate vascular smooth muscle cell motility in culture through a Src-family tyrosine kinase, Fyn (Ginnan et al., 2013). Because focal adhesions are known to serve as ERK scaffolds in contractile smooth muscle, this is an appealing possible link. Thus, at the present time, although CaMKII is clearly an important regulator of $\mathrm{Ca}^{2+}$-dependent vascular contractility, the complete molecular details of the CaMKII pathway used by contractile vascular smooth muscle to regulate contractility are not yet resolved. It is clear, however, that these details represent considerable untapped potential as future therapeutic targets for the modulation of $\mathrm{Ca}^{2+}$-dependent vascular contractility and hence blood pressure. Additionally, the wealth of information on isoform specific effects of CaMKII, especially the gamma G-2 variant, offers the potential of considerable tissue and smooth muscle phenotype specificity of such therapeutics.

2. Pathways Leading to Changes in Actin Availability for Interaction with Myosin. In contrast to the pathways described above, ex vivo studies (Walsh et al., 1994; Horowitz et al., 1996a; Dessy et al., 1998) have demonstrated that phorbol esters, or alpha agonists, by activating $\mathrm{PKC}$ can trigger increases in contractile force that in some tissues are either $\mathrm{Ca}^{2+}$ independent or cause leftward shifts in the $\left[\mathrm{Ca}^{2+}{ }_{\mathrm{i}}\right]$-force relationship. The $\mathrm{Ca}^{2+}$ dependence of phorbol ester contractions varies in different smooth muscle tissues, dependent on the isoforms of PKC present in those tissues. The alpha, beta, and gamma isoforms are calcium dependent, but delta and epsilon are calcium independent.
Thus, phorbol ester and alpha agonist-induced contractions have been described as being $\mathrm{Ca}^{2+}$ independent in experiments using the aorta of the ferret, which contains significant amounts of the epsilon isoform of PKC, but tissues containing more PKC alpha, such as the portal vein of the ferret, show an increased $\mathrm{Ca}^{2+}$ sensitivity but are still $\mathrm{Ca}^{2+}$ sensitive (Lee et al., 1999) and lead to the activation of Pathway \#3 in Fig. 3.

Pathway 3 can be activated by diacylglycerol (DAG) release, generated by the activation of GPCRs in vascular smooth muscle, or by myometrial stretch, RU486, and labor in the rat and human myometrium ( $\mathrm{Li}$ et al., 2003, 2004; Morgan, 2014). Interestingly, in the presence of extracellular $\mathrm{Ca}^{2+}\left(\mathrm{Ca}_{\mathrm{e}}\right)$, phenylephrine (PE) will activate pathways 1,3 , and 4 but in the absence of $\mathrm{Ca}_{\mathrm{e}}$ and in the absence of changes in $20 \mathrm{kda}$ light chain phosphorylation, a contraction persists in aorta of the ferret (Dessy et al., 1998). Phorbol esters give a maximal tonic contraction in the absence of changes in $20 \mathrm{kda}$ light chain phosphorylation, even in the presence of $\mathrm{Ca}_{\mathrm{e}}$ in this system (Jiang and Morgan, 1989).

When activated, Pathway \#3 leads, indirectly, to the PKC-dependent activation of MEK, a dual activity kinase that phosphorylates ERK on a tyrosine and threonine, resulting in activation of ERK. ERK activation can have multiple downstream effects, largely controlled by output-specific scaffolding proteins (see below). In contractile smooth muscle, these downstream effects include phosphorylation of the actin binding protein caldesmon. Caldesmon has been described as being functionally analogous to the troponin complex in striated muscle in that it blocks the access of myosin to actin and hence impairs crossbridge cycling. The C-terminal end of caldesmon is responsible for the direct inhibition of myosin ATPase activity (Sobue et al., 1982; Bryan et al., 1990; Wang et al., 1991). Investigators have demonstrated that the interaction of the actin-binding domain of caldesmon with actin is responsible for the inhibition the actomyosin ATPase (AMATPase) (Velaz et al., 1990) by decreasing the rate of Pi release by $80 \%$ (Alahyan et al., 2006).

Caldesmon has an $\mathrm{NH}_{2}$-terminal myosin-binding domain, in addition to the COOH-terminal actinbinding domain, and thus, in theory, could crosslink actin and myosin (Goncharova et al., 2001). However, Lee et al. (2000b) also observed a tethering effect of the $\mathrm{N}$-terminal region of caldesmon to myosin that has the proposed agonist-dependent functional effect of positioning caldesmon so that its C-terminal end no longer inhibits myosin activity. The binding of caldesmon to myosin is regulated by $\mathrm{Ca}^{2+}$-calmodulin, whereas the interaction with actin is regulated by ERK phosphorylation at Ser789 on caldesmon (Hemric et al., 1993; Patchell et al., 2002).

In general, in most systems it appears that phosphorylation of caldesmon on Ser789 by ERK, PAK, or other 
serine kinases can reverse caldesmon-mediated inhibition of myosin ATPase activity (Childs et al., 1992; Foster et al., 2000; Kim et al., 2008a) (Pathway \#3, Fig. 3). However, results from mechanical experiments examining caldesmon function are variable. In smooth muscle from caldesmon KO mice, compared with WT controls, both the rate of force activation and the steadystate force in response to depolarization, phorbol esters, and carbachol were similar, but the rate of force relaxation was reduced (Guo et al., 2013). In contrast to these results, an siRNA-induced decrease in caldesmon expression lowered both isometric force and muscle shortening velocity (Smolock et al., 2009).

In cultured smooth muscle cells, p42/44 MAPK has been clearly demonstrated to phosphorylate caldesmon at Ser789 (Hedges et al., 2000), but for agonist activation of intact smooth muscle, the kinase responsible for caldesmon phosphorylation remains a matter of controversy or may involve different kinases in different settings (Wang, 2008). In skinned smooth muscle strips, ERK-induced phosphorylation of caldesmon did not alter the force-Ca ${ }^{2+}$ relationship (Nixon et al., 1995). Porcine carotid artery preparations did not display detectable phosphorylation of caldesmon at the ERK sites during phorbol ester stimulation, (D'Angelo et al., 1999), but PAK phosphorylation at Thr627, Ser631, Ser635, and Ser642 was demonstrated to reduce caldesmon's inhibition of the AMATPase (Hamden et al., 2010). On the other hand, ERK-mediated phosphorylation of caldesmon at 789 has been clearly shown in ferret aorta preparations as well as mouse aorta and rat myometrium. Furthermore, although an increase in caldesmon phosphorylation was observed by Katoch and Moreland (1995) in porcine carotid artery during both depolarization and histamine stimulation, experiments using inhibitors suggested that a second kinase in addition to ERK also phosphorylates caldesmon (Gorenne et al., 2004).

In contrast to the myosin regulatory pathways, this is a relatively untapped area of investigation for the discovery of new target molecules with therapeutic potential. The relative importance of these pathways are definitely tissue and species specific. Interestingly, the strongest evidence of the importance of these pathways appears to have come from myometrial smooth muscle in the setting of preterm labor $(\mathrm{Li}$ et al., 2003, 2004, 2007, 2009). Thus, the potential is there for novel and possibly quite specific therapeutic targets within these pathways.

3. Tyrosine Phosphorylation of Smooth Muscle Proteins. The vast majority of known protein phosphorylation events in the contractile, differentiated smooth muscle cell are serine/threonine events. Where phosphotyrosine screening with immunoblots of contractile vascular as well as myometrial (Li et al., 2007, 2009; Min et al., 2012) smooth muscle tissue has been performed, the reactive bands have been almost exclusively focal adhesion-associated proteins. These tyrosine phosphorylations are largely sensitive to Src inhibitors, pointing to the presence of focal adhesion remodeling in nonproliferating, nonmigrating smooth muscle (Poythress et al., 2013; Ohanian et al., 2014; Zhang et al., 2015). These mechanisms have been especially studied in vascular and airway smooth muscles, resulting in pathways extending from Pathway \#4 (Fig. 3). These mechanisms will be discussed in further detail in section $\mathrm{V}$ below.

4. Calcium Sensitization of the Contractile Apparatus. When our group first (Bradley and Morgan, 1982, 1985) measured intracellular Ca levels $\left(\left[\mathrm{Ca}^{2+}\right]_{\mathrm{i}}\right)$ in dVSM with the photoprotein aequorin, we noticed that agonists often cause tonic contractions with only transient increases in $\left[\mathrm{Ca}^{2+}\right]_{\mathrm{i}}$ or differing magnitudes of $\left[\mathrm{Ca}^{2+}\right]_{\mathrm{i}}$, reflecting apparent changes in " $\mathrm{Ca}^{2+}$ sensitivity" of the contractile apparatus (Bradley and Morgan, 1985). This dissociation between $\left[\mathrm{Ca}^{2+}\right]_{\mathrm{i}}$ and force has been confirmed with many agonists and many different $\mathrm{Ca}^{2+}$ indicators in contractile smooth muscle tissues and with permeabilized smooth muscle preparations where leftward shifts in the $\mathrm{Ca}^{2+}$-force relationship in response to agonists and various agents are seen (Ruegg and Pfitzer, 1985; Somlyo et al., 1999). Mechanistically, we now have molecular explanations for this phenomenology. Changes in the apparent $\mathrm{Ca}^{2+}$ sensitivity of the contractile apparatus have been partially explained by the ability of agonists to regulate the activity of myosin phosphatase (MP) (Somlyo and Somlyo, 2003) (Pathway \#2, Fig. 3), partially by the ability of ERK to regulate the action of caldesmon $(\mathrm{CaD})$ to inhibit acto-myosin interactions (Kordowska et al., 2006) (Pathway \#3, Fig. 3) and clearly also by yet to be defined pathways.

\section{B. Subcellular Spatial Organization of Signaling Pathways}

The complexity of signaling pathways in the smooth muscle cell raises the issue of how kinases connect with their complex specific upstream activators and downstream substrates in an agonist-specific manner within the three-dimensional space of the interior of a cell. Scaffold proteins are now recognized to play important roles in coordinating mammalian signal transduction (Morrison and Davis, 2003; Kolch, 2005). Protein scaffolds are defined as docking platforms that colocalize components of kinase cascades and facilitate activation of the kinases (McKay and Morrison, 2007). The scaffolds themselves generally lack enzymatic activity but promote specific outcomes of the pathway. Protein scaffolds can be thought of as "traffic cops" in what would otherwise be the chaos of multiple competing intracellular signaling pathways. Because scaffold proteins add specificity to the cellular pathways, they also present very attractive targets for drug discovery programs. Two major types of scaffolds relevant for 
the smooth muscle cell are ERK scaffolds and scaffolds for regulators of myosin phosphatase.

1. Extracellular Regulated Kinase Scaffolds (Calponin, SmAV, Paxillin, Caveolin, FAK, IQGAP). ERK is known to often be targeted to the intranuclear space in proliferative cells and to regulate nuclear signaling, especially to transcription factors (Dhanasekaran et al., 2007). In the smooth muscle cell these pathways can lead to a proliferative phenotype for the smooth muscle cell. These pathways will not be discussed here, but rather we will focus on those most relevant for the fully differentiated contractile cell. Even so, much of this work has been performed using cell culture models and no doubt needs further work in specific contractile smooth muscle tissue systems.

Calponin is a bit of an enigma and its function in smooth muscle is still debated. It has been reported to serve both cytoskeletal and signaling functions (Winder and Walsh, 1990; Birukov et al., 1991; Menice et al., 1997; Leinweber et al., 1999a,b, 2000; Appel et al., 2010). Both PKC and CAM kinase II phosphorylate calponin at Ser175 (Winder and Walsh, 1990), and after phosphorylation, calponin loses its ability both to bind actin and inhibit the AMATPase (Winder et al., 1993). Calponin has been reported directly to regulate contractility (el-Mezgueldi and Marston, 1996; Obara et al., 1996; Winder et al., 1998; Takahashi et al., 2000; Je et al., 2001; Szymanski et al., 2003) but others have reported negative results (Matthew et al., 2000). Calponin phosphorylation increases during carbachol stimulation of smooth muscle (Winder et al., 1993). Consistent with a physiologic role for calponin in the regulation of contractility are results in skinned smooth muscle; the addition of exogenous calponin reduces both $\mathrm{Ca}^{2+}$ activated force (Horowitz et al., 1996b; Obara et al., 1996) and maximal shortening velocity (Jaworowski et al., 1995). In the smooth muscle isolated from calponin $\mathrm{KO}$ mice, compared with WT controls, muscle-shortening velocity is significantly higher, but there is no difference in the force produced by $\mathrm{Ca}^{2+}$, carbachol, or phorbol esters (Matthew et al., 2000). However, the addition of exogenous calponin reduces force in skinned single smooth muscle cells, and the Ser175Ala calponin mutant has no effect on force (Horowitz et al., 1996a). In intact smooth muscle during agonist-induced activation, calponin redistributes from the contractile filaments to the cell surface, which is attenuated with the inhibition of PKC (Parker et al., 1994, 1998; Gallant et al., 2011). Thus, these results are consistent with a role for calponin in the regulation of smooth muscle contraction; agonist stimulation leads to the activation of PKC, which phosphorylates calponin at Ser-175 to decrease calponin's interaction with actin to relieve calponin's inhibition of the AMATPase.

Three isoforms exist for calponin. h1CaP/CNN1/basic calponin is one of the most specific and rigorous markers for the differentiated smooth muscle phenotype. h2CaP/ CNN2/neutral calponin and h3/acidic CaP/CNN3/aCaP are more widely distributed but appear to also be expressed in some smooth muscles (Takahashi et al., 1988; Strasser et al., 1993; Applegate et al., 1994). Work in our group has led us to propose that calponin is an adaptor protein for ERK (Leinweber et al., 1999a,b; Appel et al., 2010). Antisense knock down of calponin (Je et al., 2001) led to decreased ERK activity and contractile force after alpha agonist activation but not after a depolarizing stimulus. Also, protein chemistry studies and cellular immunoprecipitation studies demonstrated that $\mathrm{CaP}$ directly binds both PKC and ERK and in intact vascular smooth muscle cells (Leinweber et al., 2000) and is bound to the thin filaments but translocates to the cortex of the cell in response to alpha agonists (Parker et al., 1998). A detailed model has been suggested where agonists activate PKC, which phosphorylates $\mathrm{CaP}$, releasing it from the thin filaments (Kim et al., 2008a). Colocalization of ERK and CaP is seen in unstimulated vascular smooth muscle cells and agonist-activation leads to the translocation of a PKC/ CaP/ERK complex to the cell cortex, likely meeting up with SmaV (see below), Raf, and MEK, which leads to the activation of ERK, at which point it is seen to return to the contractile filaments and $\mathrm{CaD}$ phosphorylation of the ERK sites is observed (Khalil and Morgan, 1993).

$\mathrm{SmAV}$ is the smooth muscle isoform of a major scaffolding protein supervillin (Pestonjamasp et al., 1997). SmAV was initially cloned and identified SmAV as a CaP binding partner in a two-hybrid assay with $\mathrm{CaP}$ as bait (Gangopadhyay et al., 2004), and it was found that SmAV acts as an ERK scaffold, leading to the regulation of $\mathrm{CaD}$ phosphorylation (Gangopadhyay et al., 2009). Data have been published indicating that $\mathrm{CaP}$ (Menice et al., 1997), SmAV (Gangopadhyay et al., 2004) and $\mathrm{CaV}$ (Je et al., 2001) function as scaffolds coordinating Pathway \#3.

Paxillin, better known as a focal adhesion protein, is also known to bind the classic ERK "signaling module" of Raf, MEK, and ERK (McKay and Morrison, 2007). In quiescent cultured cells, paxillin is constitutively associated with MEK, but Ishibe et al. (2003) showed that when cells are stimulated with HGF, Src-mediated phosphorylation of paxillin at Y118 leads to the recruitment of ERK, followed by Raf, which leads to ERK phosphorylation and activation. Shortly thereafter focal adhesion kinase (FAK) is recruited to the complex, leading to FA remodeling in both cultured cell systems and airway smooth muscle (Zhang et al., 2015). Thus, paxillin provides a signaling hub in the vicinity of focal adhesions that can have specific cytoskeletal outcomes.

Caveolin is an extensively studied protein but there are still many mysteries regarding its function. A caveolin-associated protein has also discovered and named cavin (Liu and Pilch, 2008; Ding et al., 2014; Kovtun et al., 2015). The exact way in which caveolin and cavin interact and the role of cavin specifically in smooth muscle is not yet clear; however, a cavin 
knockout mouse has been produced (Sward et al., 2014). In this knockout animal not only were arterial expression of cavin-1, cavin-2, and cavin-3 reduced but also all isoforms of caveolin were reduced. As a result, caveolae were absent from both smooth muscle and endothelial cells. An enhanced contractile response to an alpha 1 adrenergic agent was seen, but was likely to be due to the increased thickness of the vascular wall. In contrast, myogenic tone was essentially absent. Surprisingly, blood pressure of the knockout mouse was well maintained, presumably due to opposing influences from smooth muscle and endothelial effects.

Inhibition of caveolin function by a caveolin decoy peptide or by methyl-beta-cyclodextrin has been shown to disrupt ERK activation in vascular smooth muscle (Je et al., 2004). Work using cultured vascular smooth muscle cell models has suggested that caveolin-mediated scaffolding of ERK leads to different functional outputs than actin/calponin-mediated scaffolding (Vetterkind et al., 2013). This concept has not yet been pursued in contractile smooth muscle but illustrates the idea of scaffold proteins regulating the output of kinase cascades toward separate purposes and serving as traffic cops for complex cellular signaling pathways.

IQGAP (IQ motif containing GTPase activating protein) is an ERK-binding and actin-binding protein that has been extensively studied in nonmuscle systems but little studied in smooth muscle systems. In cultured vascular smooth muscle cells, knockdown of IQGAP prevents the phosphorylation and activation of an actinassociated pool of ERK in response to PKC activation. Proximity ligation assays demonstrated direct tethering of ERK1/2 to actin by IQGAP. Interestingly caveolin is also required for activation of this pathway unless ERK is already associated with actin. Caveolin appears to be required specifically for upstream C-raf activation (Vetterkind et al., 2013).

2. Myosin Phosphatase Scaffolds. Myosin regulation is discussed in detail in section IV below, but multiple pathways have been suggested to coordinate signaling associated with myosin phosphatase (MP), and hence, myosin activity (Pathway \#2, Fig. 1), and it seems likely that scaffolds play a role to regulate/facilitate these pathways. One MP putative scaffold, M-RIP, also called p116 ${ }^{\mathrm{RIP}}$, is thought to link active Rho/ROCK to the inhibition of MP (Surks and Mendelsohn, 2003; Mulder et al., 2004; Koga and Ikebe, 2005). Vetterkind and Morgan (2009) reported that another scaffold/adaptor protein, Par-4, also regulates myosin phosphatase activity in contractile smooth muscle. We have described a "padlock" model to explain the actions of Par-4, whereby binding of Par-4 to MYPT1 activates MP. This is postulated to occur by the physical blockade by Par-4 of the MYPT1 inhibitory phosphorylation sites. Conversely, this model indicates that inhibitory phosphorylation of MYPT1 by Zipper-interacting protein kinase requires "unlocking" of the blockade by phosphorylation and displacement of Par-4 (Vetterkind et al., 2010). Whether M-RIP and Par-4 facilitate or antagonize each other's actions is not known.

The complexity of this system is impressive, but it is expected that the multiple scaffolding proteins and signaling molecules involved in regulating myosin phosphorylation will lead to the development of rational and selective therapeutic approaches to cardiovascular disease.

\section{Link to Hypertension}

We describe here a number of pathways by which vascular smooth muscle contraction and stiffness are directly regulated and hence will affect blood pressure. It should be mentioned that many other indirect pathways are also involved, with a major mechanism being the development of inflammation and subsequent reduction-oxidation reaction (REDOX) signaling pathways (Sorescu et al., 2001; Loirand and Pacaud, 2014). These pathways are triggered by angiotensin-induced signaling, and as a result, inhibitors of the effects of and production of angiotensin are major ways of regulating blood pressure, including blood vessel contraction. For further details, we refer you to Mehta and Griendling (2007) for a review of this topic.

\section{Potential Novel Therapeutic Targets/Approaches / Critical Analysis of Pathway-Specific Inhibitors}

1. Rho Kinase Inhibitors. Y27632, the first ROCK inhibitor described, decreases blood pressure in 11-Deoxycorticosterone acetate (DOCA)-salt rat model of hypertension. A similar effect was obtained with the newer ROCK inhibitors fasudil, SAR07899, in other animal models of hypertension, including the spontaneously hypertensive rat (SHR), angiotensin II-induced hypertension in several animals, and L-NG-Nitroarginine Methyl Ester (L-NAME)-induced hypertension (Uehata et al., 1997; Mukai et al., 2001; Kumai et al., 2007; Lohn et al., 2009). Of note, this class of inhibitors also has a major part of their effect on hypertension through inhibition of inflammatory pathways and cardiovascular remodeling. For more details we refer you to a recent review by Loirand and Pacaud (2014).

2. Endothelin Inhibitors. The endothelin pathway, linked to PLC and ERK signaling, has been identified as an effective antihypertensive target (Sandoval et al., 2014).

3. Beta Adrenergic Receptor Mediated Inhibition. Of interest is the fact that beta receptor mediated relaxation of vascular smooth muscle has been reported to decline with age in both the human and animal models. In aortas from Fischer 344 rats, an increase in the level of G-protein receptor kinase-2, which desensitizes the beta adrenergic receptor by phosphorylation of the receptor has been reported to increase with age (Schutzer et al., 2005), and thus inhibitors of G-protein 
receptor kinase-2 may promote beneficial restoration of beta receptor mediated vasodilation.

\section{Regulation of Smooth Muscle Myosin}

\section{A. Overview of Regulation of the Smooth Muscle Actomyosin ATPase and 20kda light chain Phosphorylation/Smooth Muscle Activation}

The crossbridge cycle describes the development of force through a series of complexes between actin (A), myosin (M), ATP, and its hydrolysis products, ADP and Pi (Sweeney and Houdusse, 2010) (Fig. 4 and the termination of Pathway \#1; Fig. 3). Beginning in the rigor state (AM), ATP binding to AM results in rapid dissociation of AM, forming an A+M-ATP state, and then ATP is hydrolyzed by myosin. After hydrolysis, the crossbridge enters a weakly attached, pre-powerstroke AM-ADP-Pi state, and then transitions to a strongly bound, force producing AM-ADP-Pi state. After Pi release from the AM-ADP-Pi state, the crossbridge enters a AM-ADP state, which then isomerizes to a high force generating state (AM-ADP) followed by ADP release and returning to the rigor state (AM). MgATP subsequently binds to the AM state, causing rapid crossbridge detachment, and then another crossbridge cycle commences. The duty cycle is defined as the proportion of time crossbridges spend in strongly attached states divided by the time for the total crossbridge cycle (De La Cruz and Ostap, 2004); high duty cycle motors are capable of processive movement (i.e., dynein, myosin V), whereas skeletal muscle myosin has a low duty cycle that prevents the development of an internal load from strongly bound crossbridges, which would decrease shortening velocity. Although the crossbridge cycle for all types of myosin is frequently described in this generic manner, differences exist between the kinetics of skeletal, cardiac, and smooth muscle and even within different smooth muscle tissues, requiring changes in the crossbridge cycle to explain the differences in AMATPase rates (Rosenfeld et al., 2000).

The smooth muscle AMATPase is similar to that of striated muscle, albeit the kinetics are slower. The kinetics and individual rate constants of the steps in the actomyosin ATPase have been defined in a number of studies (Rosenfeld et al., 2000; Baker et al., 2003; Haldeman et al., 2014), and similar to other myosin IIs, the ATPase is limited by phosphate release or the transition from weak to strong binding states (Haldeman et al., 2014). Both cardiac and skeletal muscle myosin is functionally on, i.e., myosin will hydrolyze ATP in the presence of actin. Smooth muscle (SM) myosin will hydrolyze ATP in the presence of actin, albeit very slowly; however, after phosphorylation of the $20-\mathrm{kDa}$ regulatory myosin light chain (RLC), the rate of hydrolysis is increased (Chacko et al., 1977; Ikebe and Hartshorne, 1985; Ikebe and Morita, 1991; Ellison et al., 2000) due to an $\sim 1000$-fold increase in the rate of product release (Sellers and Adelstein, 1985). Thus, changes in RLC phosphorylation regulate smooth muscle activation and relaxation.

In smooth muscle, in addition to RLC phosphorylation regulating the AMATPase, it also controls the structure of SM myosin and filament formation (Ikebe and Hartshorne, 1985). In the absence of RLC phosphorylation, myosin is in the $10 \mathrm{~S}$ conformation [high sedimentation velocity and low ATPase (Ikebe and Hartshorne, 1985), with the tail of myosin bending back over the head neck junction interacting with the regulatory light chain (Jung et al., 2011; Salzameda et al., 2006)]. After RLC phosphorylation, the interaction of the myosin tail with the RLC is perturbed (Jung et al., 2011), and myosin exists in an extended conformation [6S, low sedimentation velocity, high ATPase

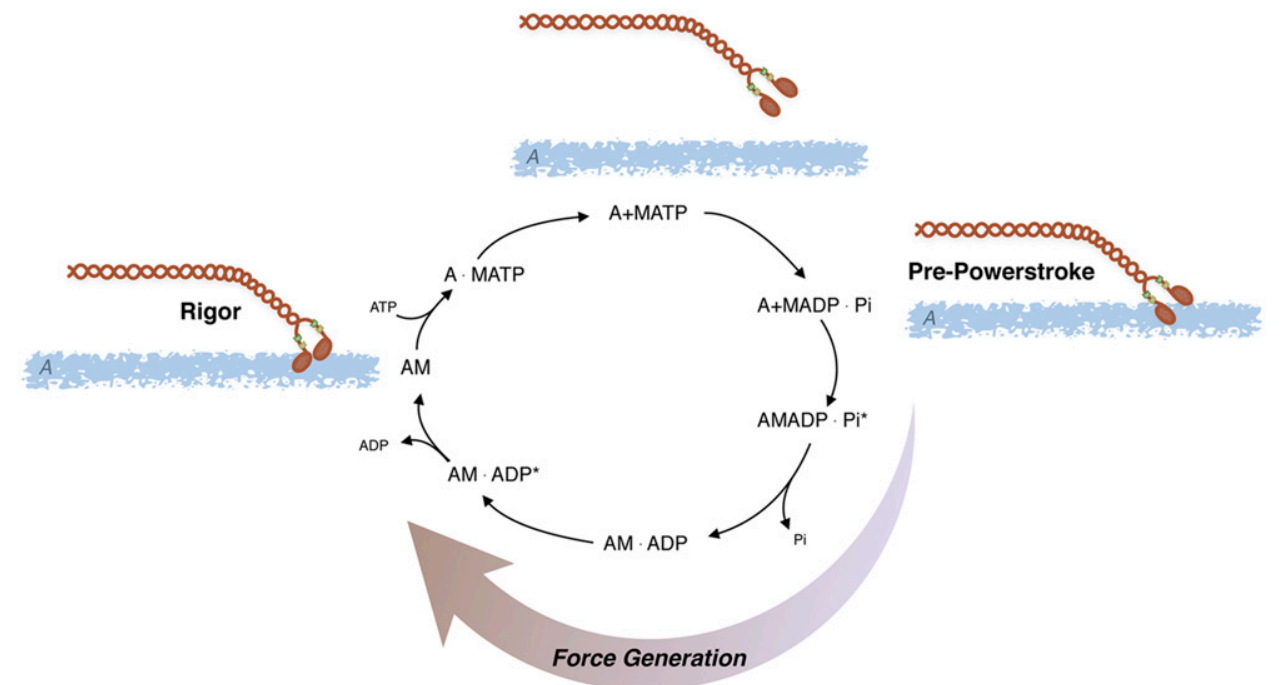

Fig. 4. AMATPase: Actomyosin ATPase cycle; ATP is hydrolyzed by myosin (M) and the subsequent interaction of myosin with actin (A) produces force and/or displacement (see text for details). 
(Ikebe and Hartshorne, 1985)] and also forms filaments (Applegate and Pardee, 1992). Other investigators have suggested that in the absence of RLC phosphorylation, the interaction of the $\mathrm{NH}_{2}$-terminal region of caldesmon (see Fig. 3 and section III.A.1) with the myosin crossbridge disrupts the interaction of the myosin head with its neck-tail region to promote a transition from the $10 \mathrm{~S}$ to the active, 6S conformation (Wang, 2008). Nonetheless, in human smooth muscle, there is significant pool of $10 \mathrm{~S}$ myosin that can be converted by changes in cellular conditions to $6 \mathrm{~S}$ myosin that can then assemble into side polar thick filaments (Milton et al., 2011). These data could suggest that in addition to the regulation of the AMATPase, changes in RLC phosphorylation could regulate the formation of myosin filaments within the smooth muscle during activation and relaxation (Pratusevich et al., 1995; Ali et al., 2007; Liu et al., 2013; Seow, 2013; Lan et al., 2015); however in vivo, the ability of RLC phosphorylation to regulate filament formation is controversial (Seow, 2015; Somlyo, 2015).

The level of RLC phosphorylation is defined by the relative activities of myosin light chain kinase (MLCK) and MLC phosphatase (Gong et al., 1992), i.e., RLC phosphorylation is related to MLCK/(MLCK+MLC phosphatase). Thus, changes in the activity of either MLCK or MLC phosphatase will change RLC phosphorylation and force or vascular tone. MLCK is regulated by $\mathrm{Ca}^{2+}$-calmodulin (Ikebe and Hartshorne, 1985), whereas MLC phosphatase activity is regulated by a number of signaling pathways (Hartshorne et al., 1998). At a constant $\left[\mathrm{Ca}^{2+}\right]$, a decrease in MLC phosphatase activity increases SM RLC phosphorylation and force to produce $\mathrm{Ca}^{2+}$ sensitization (Somlyo and Somlyo, 2003), whereas an increase in MLC phosphatase activity decreases SM RLC phosphorylation and force to produce $\mathrm{Ca}^{2+}$ desensitization (Somlyo and Somlyo, 2003).
MLC phosphatase is a holoenzyme (Hartshorne et al., 1998) consisting of a catalytic subunit, a $20-\mathrm{kDa}$ subunit of unknown function, and a myosin targeting subunit (MYPT1). Alternative splicing of a 123-bp central exon results in MYPT1 isoforms that differ by a 41-aa central insert (CI), referred to as M130 and M133 (Hartshorne et al., 1998). Additionally, alternative splicing of the $3^{\prime}$ exon (Khatri et al., 2001) is responsible for generating MYPT1 isoforms that differ by the presence or absence of a carboxy-terminal leucine zipper (LZ). Thus in humans and other species, alternative splicing generates four MYPT1 isoforms that differ by the presence or absence of a CI and LZ: CI+LZ+, CI-LZ+, CI+LZ-, CI-LZ- (Hartshorne et al., 1998).

\section{B. Guanine Nucleotide Exchange Factor Signaling, Rac/Rho, and Analysis of Inhibitors}

The Rho GTPases are within the RAS superfamily of small G proteins (Jaffe and Hall, 2005), which exist as either active GTP-bound and inactive GDP-bound forms. The conversion between active and inactive forms is controlled by guanine nucleotide exchange factors (GEFs), GTPase activating proteins (GAPs), and guanine dissociation inhibitors. The role of RhoA for the regulation of smooth muscle tone has been well described (Fig. 5; Ca2+ sensitization and Pathway \#2, Fig. 3). RhoA/Rho kinase signaling is activated by $G$ protein-coupled receptors, and the role of this pathway for the inhibition of MLC phosphatase and $\mathrm{Ca}^{2+}$ sensitization has been the subject of a number of reviews (Arner and Pfitzer, 1999; Somlyo and Somlyo, 2003; Puetz et al., 2009). The activation of Rho kinase has been demonstrated to phosphorylate CPI-17 at Thr38 (Eto et al., 1995; Kitazawa et al., 2000), PHI-1 at Thr57 (El-Touhky et al., 2005, 2006), and MYPT1 (TrinkleMulcahy et al., 1995) at both T696 and T850 (Muranyi et al., 2005). MYPT1 phosphorylation at T696 has been

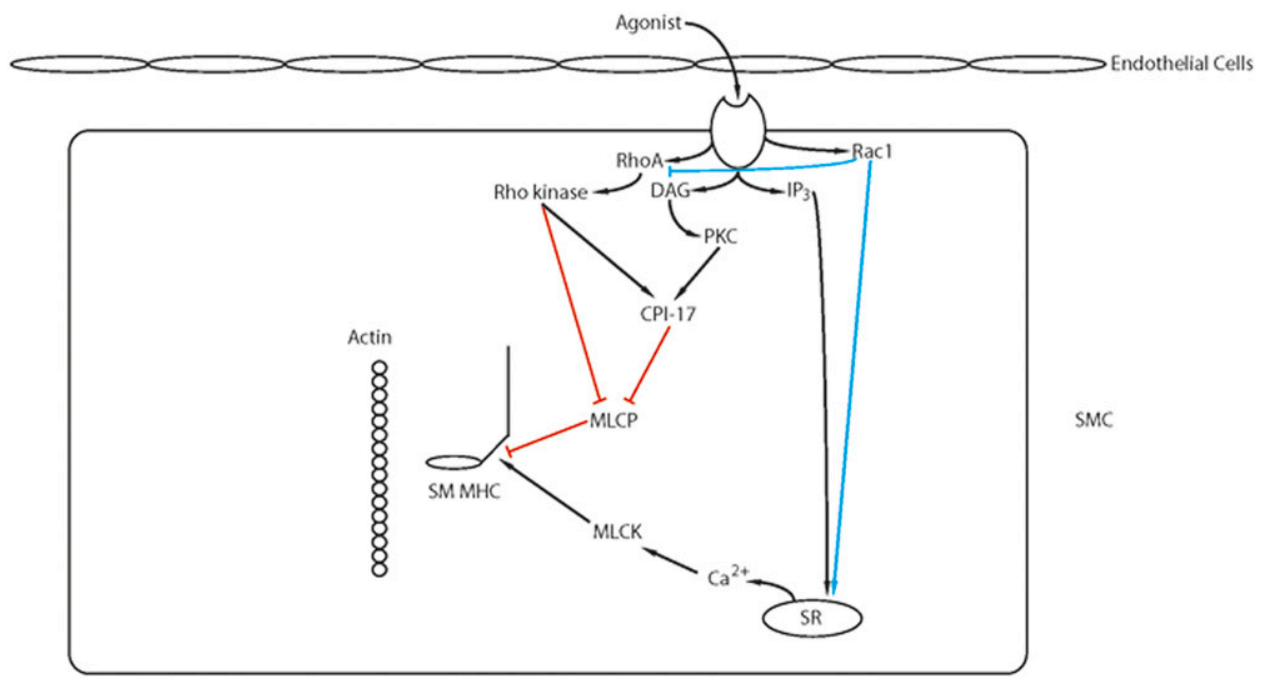

Fig. 5. $\mathrm{Ca}^{2+}$ sensitization: Agonist activation of G-protein coupled receptors activates several signaling pathways (IP 3 , RhoA/Rho kinase, PKC, RAC1) that modulate $\mathrm{Ca}^{2+}$ release from the SR and/or lead to $\mathrm{Ca}^{2+}$ sensitization of the contractile filaments (see text for details). 
demonstrated to decrease MLC phosphatase activity to increase force at a constant $\mathrm{Ca}^{2+}$ (Kitazawa et al., 1991a), whereas the phosphorylation of MYPT1 at T850 dissociates the holoenzyme, which results in a decrease in MLC phosphatase activity (Velasco et al., 2002), which produces $\mathrm{Ca}^{2+}$ sensitization. Similarly, agonist activation of G-protein-coupled receptors has been demonstrated to lead to the activation of PKC, which phosphorylates both CPI-17 at Thr38 (Eto et al., 1995; Kitizawa et al., 1991b) PHI-1 at Thr57 (Eto et al., 1999). When phosphorylated, these proteins will bind to the catalytic core of the catalytic subunit of MLC phosphatase to decrease phosphatase activity (ElToukhy et al., 2006; Eto et al., 2007). In addition to Rho kinase, Zip kinase will phosphorylate MYPT1 (MacDonald et al., 2001a) as well as CPI-17 (MacDonald et al., 2001b), and integrin-linked kinase will phosphorylate MYPT1 (Kiss et al., 2002; Muranyi et al., 2002) as well as CPI-17 and PHI-1 (Deng et al., 2002).

There are a number of studies demonstrating Rho kinase signaling mediates both MYPT1 phosphorylation and $\mathrm{Ca}^{2+}$ sensitization (Somlyo and Somlyo, 2003; Puetz et al., 2009). However, in smooth muscle of the guinea pig ilium, although inhibition of Rho kinase decreased the carbachol-induced increase in $\mathrm{Ca}^{2+}$ sensitivity, it had no effect on MYPT1 phosphorylation (Pfitzer, 2001). In this study, although staurosporin prevented MYPT1 phosphorylation, specific PKC inhibitors had no effect on the phosphorylation of MYPT1, which could suggest in this tissue Zip kinase is involved in a physiologically important signaling pathway for $\mathrm{Ca}^{2+}$ sensitization. In bladder smooth muscle, others have demonstrated that there is constitutive phosphorylation of MYPT1 at T696, which was unaffected by inhibition of either Rho kinase or PKC, whereas MYPT1 phosphorylation at T850 was primarily mediated by Rho kinase (Chen et al., 2015). These investigators generated T696A and T850A MYPT1 mutant mice to demonstrate that the MYPT1 phosphorylation at T696, but not T850, is important for increasing RLC phosphorylation and $\mathrm{Ca}^{2+}$ sensitization during the sustained phase of force maintenance. These results demonstrate that during activation of smooth muscle, the physiologically important signaling pathways mediating both MYPT1 phosphorylation as well as $\mathrm{Ca}^{2+}$ sensitization are agonist as well as tissue specific.

Although the role of RhoA in the regulation of $\mathrm{Ca}^{2+}$ sensitization of smooth muscle is established, the role of other Rho GTPases for force regulation in smooth muscle is not well defined. Pak1, a downstream target of Rac1, has been demonstrated to inhibit MLCK and relax permeabilized intestinal smooth muscle (Wirth et al., 2003). However in airway smooth muscle, both the knockout or inhibition of Pak reduces tone (Hoover et al., 2012), and Pak3 has been shown to induce a $\mathrm{Ca}^{2+}$ independent contraction of permeabilized smooth muscle (Van Eyk et al., 1998; McFawn et al., 2003).
Furthermore, Pak also has been demonstrated to phosphorylate CPI-17 (Takizawa et al., 2002). Recently, the role of Rac1 in force regulation has been further delineated (Rahman et al., 2014). Using a smooth muscle-specific, conditional Rac1 KO, these investigators demonstrated that the decrease in Rac1 reduced force in both bladder and saphenous arterial smooth muscle. Furthermore, the inhibition of Rac1 with EHT1864, which affects nucleotide binding, decreased the $\mathrm{Ca}^{2+}$ transient and force produced in response to depolarization, agonist activation, and activation of PKC. However, inhibition of Rac1 with NSC23766, which blocks the interaction of Rac1 with GEFs, decreased force for phenylephrine ( $\alpha$-agonist) activation but increased the force produced by stimulation with both prostaglandin F2 $\alpha$ and thromboxane (U46619). These results suggest that Rac1 signaling is agonist dependent and can result in either an enhancement of the $\mathrm{Ca}^{2+}$ transient and force or an inhibition of $\mathrm{Ca}^{2+}$ sensitization.

The well documented role of RhoA/Rho kinase signaling for the inhibition of MLC phosphatase and $\mathrm{Ca}^{2+}$ sensitization (Somlyo and Somlyo, 2003) could suggest that $\mathrm{Ca}^{2+}$ sensitization of vascular smooth muscle cells contributes to the molecular mechanism for the increase in vascular tone and/or systemic vascular resistance that produces hypertension. Consistent with this hypothesis are the results demonstrating that in SHR compared with control rats, both the sensitivity to G-protein-coupled agonists and the magnitude and sensitivity of $\mathrm{Ca}^{2+}$ sensitization are increased (Satoh et al., 1994) as well as studies defining the role of $\mathrm{G}_{12^{-}}-\mathrm{G}_{13^{-}}$ induced activation of Rho kinase-mediated $\mathrm{Ca}^{2+}$ sensitization for the development of DOCA salt-sensitive hypertension (Wirth et al., 2008). Furthermore, the infusion of the Rho kinase inhibitor Y-27632 reduced blood pressure in normal Wistar rats, as well as several animal models of hypertension including the SHR and DOCA salt-sensitive rat models of hypertension (Uehata et al., 1997), as well as L-NG-Nitroarginine Methyl Ester (L-NAME)-induced hypertension (Seko et al., 2003). Additionally, the expression and activity of Rho kinase are higher in vascular smooth muscle isolated from the SHR compared with controls (Mukai et al., 2001), and the specific Rho kinase inhibitor fasudil (Uehata et al., 1997) reduced blood pressure in the SHR model of essential hypertension (Mukai et al., 2001).

Rho/Rho kinase signaling has also been implicated as an important contributor for the regulation of vascular tone in other mammals; the Rho kinase inhibitor HA1077 has been shown to dilate canine coronary arteries (Asano et al., 1989). Furthermore, Rho kinase is upregulated in a porcine model of coronary vasospasm, and in this model, inhibition of Rho kinase with Y-27632 decreases coronary vasospasm (Kandabashi et al., 2000). In humans, fasudil has been demonstrated 
to be effective in treating the cerebral vasospasm associated with subarachnoid hemorrhage (Tanaka et al., 2005; Kim et al., 2006), and fasudil is approved for treating patients with pulmonary hypertension (Archer et al., 2010). In humans, Masumoto et al. (2001) demonstrated that brachial artery infusion of fasudil did not decrease systemic blood pressure, but did produce a dose-dependent increase in forearm blood flow in hypertensive patients, but not controls without hypertension. Sodium nitroprusside, on the other hand, resulted in comparable increases in forearm blood flow in both hypertensive and normal humans. These results suggest that an increase in RhoA/Aho kinase signaling may be involved in the molecular mechanism that produces essential hypertension, and development of drugs that target this pathway could represent an effective, novel class of therapeutic agents.

\section{Phenotypic Switching of Contractile Proteins during Development and Disease: Role of MYPT1 in $\mathrm{Ca}^{2+}$ Sensitization/Desensitization}

1. Smooth Muscle Myosin Heavy Chain. Four isoforms of the smooth muscle myosin heavy chain (SM MHC) are produced by alternative splicing of a single gene. Alternative splicing of a $5^{\prime}$-site produces two isoforms that express a unique aa sequence of either $43(\mathrm{SM} 1,204 \mathrm{kDa})$ or $9(\mathrm{SM} 2,200 \mathrm{kDa})$ residues at the carboxy terminus of the SM MHC tail (Babij and Periasamy, 1989; Nagai et al., 1989), whereas alternative splicing of a 21-bp insert produces a difference of 7 aa near the ATP binding site of the SM MHC (Kelley et al., 1993). Although SM1 or SM2 homodimers are more common than herterodimers in a single myosin rod, SM1 and SM2 homodimers will copolymerize and assemble into side polar thick filaments (Rovner et al., 2002). Estrogen has been demonstrated to increase the ratio of SM1/SM2 expression, and this shift in the expression of SM1 MHC isoform has been suggested to contribute to changes in the sensitivity to the agonist norepinephrine and $\mathrm{KCl}$ depolarization (Paul et al., 2007). However, others have demonstrated in the motility assay, there is no difference in the ability of SM1 and SM2 to translate actin (Rovner et al., 2002). These investigators also demonstrated that although there is no difference in the length of SM1 and SM2 filaments, the differences in SM1 and SM2 at the carboxy terminus of the myosin tail influenced filament packing and stability; SM1 filaments have greater stability (Rovner et al., 2002). Consistent with these results, others have demonstrated that the smooth muscle thick filaments of the SM2 KO are similar in length to that of WT mice, but the SM1 thick filaments isolated from SM2 KO mice are smaller in diameter and there are fewer thick filaments per high-powered field (Chi et al., 2008). However, despite the decrease in the number of smooth muscle myosin thick filaments and a concomitant decrease in the expression of SM1, the force produced by both $\mathrm{KCl}$ depolarization and carbachol activation was higher in the SM2 KO (Chi et al., 2008).

The other SM MHC isoform is due to a 7-aa insert at the amino terminus, near the ATP binding site of the SM MHC (Kelley et al., 1993). The 7-aa insert is expressed in both SM1 and SM2 SM MHCs, and the SM MHC with the 7-aa insert has been referred to as SMB, whereas SM MHC lacking the insert is SMA (Kelley et al., 1993). SMB, when compared with SMA, has been demonstrated to have a twofold higher AMATPase activity and a 2.5-fold faster velocity of actin translation in the in vitro motility assay (Kelley et al., 1993). Whether the presence of the insert confers a functional difference to the mechanical properties of smooth muscle was assessed using bladder smooth muscle isolated from a transgenic mouse line; the maximum velocity of muscle shortening of bladder smooth muscle strips from WT $(\mathrm{SMB}+/+)$ is higher than that of either of heterozygous (SMB+/-) animals or the SMB KO mice (Babu et al., 2001; Karagiannis et al., 2004). Further analysis of the mechanical responses of $\mathrm{Ca}^{2+}$-activated skinned bladder strips to elevated $\mathrm{Pi}$ and ADP suggested that the lower shortening velocity of the SMA isoform is due to a slower rate of ADP dissociation or an additional force producing isomerization of the AM-ADP state in the crossbridge cycle (Karagiannis et al., 2003). These results demonstrate that the insert near the SM MHC ATP binding site will alter the mechanical properties of smooth muscle, and the duty cycle of SMA should be higher than SMB, which would be predicted to increase vascular tone and/ or vascular resistance and thus blood pressure. Consistent with this prediction are the results demonstrating that compared with WT mice, the isometric force for mesenteric vessels of SMB KO mice was increased (Babu et al., 2004). However, the blood pressure of SMB KO animals compared with WT controls has not been reported, and whether SMA/SMB isoform expression is altered during hypertension has not been investigated.

2. ELC17. For the $17-\mathrm{kDa}$ essential myosin light chain (ELC17), alternative splicing of exon six, which encodes $44 \mathrm{bp}$, also produces two isoforms, which differ in the expression of 9 aa at the carboxy terminus of the ELC17 (Nabeshima et al., 1987; Lenz et al., 1989; Hasegawa and Morita, 1992). Exon 6 is excluded in the nonmuscle, more basic isoform (ELC17a), whereas exon 6 is included in the smooth muscle, more acidic isoform (ELC17b) (Hasegawa and Morita, 1992; Kelley et al.,1993). Changes in the ELC17 could affect the stiffness of the SM MHC lever arm, and thus myosin step size and or unitary force. However for ELC17 isoforms, the motility assay does not show any difference in the velocity of actin translocation (Kelley et al., 1993; Quevillon-Cheruel et al., 2000). Nonetheless in fast smooth muscle, the expression of ELC17a and SMA 
is higher than in slow smooth muscle (Malmqvist and Arner, 1991).

3. MYPT1. Alternative mRNA splicing produces four splice variant MYPT1 isoforms, formed by the presence or absence of a 43 aa central insert (CI+/-) and carboxy-terminal leucine zipper ( $\left.\mathrm{LZ}_{+} /-\right)$. The expression of the CI (residues 512-552) (Shimizu et al., 1994) is both developmentally regulated and tissue specific (Dirksen et al., 2000), and phosphorylation of MYPT1 at Thr696 has been demonstrated to inhibit phosphatase activity (Ichikawa et al., 1996). Although there is evidence that the CI+ MYPT1 isoform may be preferentially phosphorylated during $\mathrm{Ca}^{2+}$ sensitization (Richards et al., 2002), a role for the CI for the regulation of smooth muscle contractile properties has not been established. The MYPT1 LZ+ isoform is generated by the exclusion of a 3 ' - to 31-bp exon, whereas exon inclusion generates a LZ- MYPT1 isoform (Khatri et al., 2001). The aa sequence of the MYPT1 LZ domain is identical from avians to mammals and $75 \%$ identical in mammals and worms (Khatri et al., 2001), which could suggest that this domain is important for the regulation of MLC phosphatase activity. Surks et al. (1999) were the first to demonstrate an important functional role for the MYPT1 LZ domain. These investigators demonstrated that the LZ MYPT1 domain was important for the interaction of MYPT1 and PKG (Surks et al., 1999; Surks and Mendelsohn, 2003). Subsequently it has been demonstrated that PKG interacts with the LZ domain (Lee et al., 2007; Sharma et al., 2008) as well as a MYPT1 domain between aa 888 and 928 (Given et al., 2007; Sharma et al., 2008), but the LZ domain is necessary for the PKG-mediated activation of the MLC phosphatase during $\mathrm{Ca}^{2+}$ desensitization (Fig. 6,; $\mathrm{Ca}^{2+}$ desensitization) and/or nitric oxide (NO)-mediated vasodilatation (Huang et al., 2004). PKG phosphorylates MYPT1 at Ser695 and Ser849, which excludes the Rho kinase-mediated MYPT1 phosphorylation at Thr696 and Thr850, to prevent a Rho kinase-mediated decrease in MLC phosphatase activity (Wooldridge et al., 2004; Nakamura et al., 2007), but MYPT1 phosphorylation at these sites by PKG does not increase MLC phosphatase activity (Nakamura et al., 2007). The mechanism for the increase in MLC phosphatase activity during NO/cGMPmediated $\mathrm{Ca}^{2+}$ desensitization was recently defined; PKG phosphorylates only LZ+ MYPT1 isoforms at Ser668 (Yuen et al., 2011, 2014), and the Ser668 MYPT1 phosphorylation increases the activity of MLC phosphatase (Yuen et al., 2011; Yuen et al., 2014). MYPT1 LZ+/- isoform expression is developmentally regulated, tissue specific (Khatri et al., 2001; Payne et al., 2006), and modulated in animal models of heart failure (Karim et al., 2004; Chen et al., 2006; Ararat and Brozovich, 2009; Han and Brozovich, 2013), preeclampsia (Lu et al., 2008), portal hypertension (Payne et al., 2004; Lu et al., 2008), pulmonary hypertension (Konik et al., 2013), nitrate tolerance (Dou et al., 2010), and sepsis (Reho et al., 2015). Furthermore, a decrease in LZ+ expression decreases the sensitivity to NOmediated vasodilatation (Huang et al., 2004; Yuen et al., 2011, 2014). Thus, the molecular mechanism that underlies the differential response of the vasculature to NO and NO-based vasodilators is in part due to differential expression of LZ+/- MYPT1 isoforms, and furthermore, changes in the relative $\mathrm{LZ}+/-$ expression could tune the vasculature between a low-resistance vascular bed (relatively vasodilated), which is NO responsive and Rho kinase/PKC resistant, and a highresistance vascular bed (relatively vasoconstricted) that is resistant to $\mathrm{NO}$ but responsive to Rho kinase/PKC.

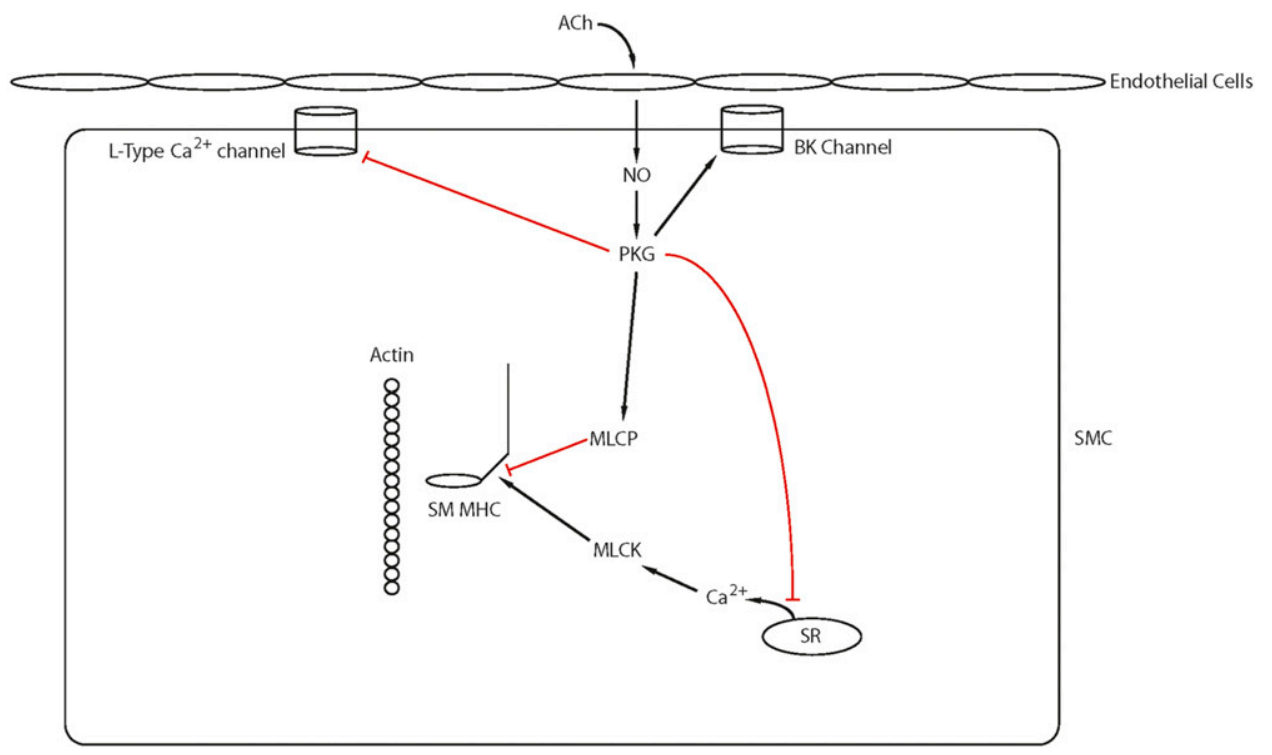

Fig. 6. $\mathrm{Ca}^{2+}$ desensitization: ACh stimulation of muscarinic receptors on the vascular endothelium leads to the production of NO, and NO diffuses into smooth muscle cells to activate guanylate cyclase. The NO/cGMP signaling pathway relaxes smooth muscle by both decreasing intracellular $\mathrm{Ca}^{2+}$ and activating MLC phosphatase, which results in $\mathrm{Ca}^{2+}$ desensitization of the contractile filaments (see text for details). 
The importance of the LZ domain of MYPT1 for the regulation of vascular tone has been established using two different transgenic mice. Michael et al. (2008) produced a transgenic mouse with mutations in the LZ domain of PKG, which disrupts the interaction of PKG with MYPT1. Compared with control littermates, the vascular smooth muscle isolated from these mice were less sensitive to NO-mediated vasodilatation, and thus these mice were hypertensive. In addition and also consistent with an important role for LZ+ MYPT1 isoform expression for the regulation of vascular tone are the results demonstrating that transgenic mice expressing only the LZ+ MYPT1 isoform are more sensitive to NO-mediated relaxation and hypotensive compared with WT littermates (Reho et al., 2015).

Because NO-mediated vasodilation is a fundamental property of the vasculature (Furchgott and Zawadzki, 1980; Furchgott, 1999), preservation of the normal response to NO by maintaining the normal LZ+ MYPT1 isoform expression could improve the outcome for the treatment of diseases of the vasculature. For the treatment of heart failure, both ACE inhibitors (Chen et al., 2006; Chen and Brozovich, 2008) and ARBs (Ararat and Brozovich, 2009) have been demonstrated to preserve both LZ+ MYPT1 expression and the sensitivity to NO-mediated vasodilatation, which could underlie the beneficial effects of inhibition of angiotensin II signaling in the treatment of heart failure compared with other vasodilators (Pfeffer et al., 1992; Yusuf et al., 1992, 2000; Pitt et al., 2000; Granger et al., 2003). Additionally, differential expression of LZ+/MYPT1 isoforms in patients with heart failure could underlie the mortality benefit of ACE inhibitors in white compared with black patients. ACE inhibitors reduced mortality in white patients, whereas in black patients, although ACE inhibitors did not show a benefit, treatment with the combination of hydralazine and isosorbide dinitrate reduced mortality (Carson et al., 1999).

The regulation for LZ+/- MYPT1 expression is unknown, but LZ+/- MYPT1 expression is both developmental regulated and tissue specific (Khatri et al., 2001; Payne et al., 2006), as well as modulated during disease states (Karim et al., 2004; Payne et al., 2004; Lu et al., 2008; Fisher, 2010). During heart failure (HF), p42/ 44 MAP kinase is activated and the expression of LZ+ MYPT1 decreases (Ararat and Brozovich, 2009), and further losartan therapy prevents the activation of $\mathrm{p} 42 / 44 \mathrm{MAP}$ kinase and preserves LZ+ MYPT1 expression (Ararat and Brozovich, 2009). Additionally, the expression of LZ+ MYPT1 isoforms as well as Tra- $2 \beta$, an atypical member of RNA binding proteins, are higher in fast (phasic) compared with slow (tonic) smooth muscle, and in an animal model of portal hypertension, Tra- $2 \beta$ is downregulated coincident with the decrease in LZ+ MYPT1 expression (E23 exon exclusion) and the shift to the expression of LZ- MYPT1 isoforms (Shukla and Fisher, 2008). These investigators demonstrated both that Tra- $2 \beta$ binds to E23 and transactivates E23 splicing (exon inclusion) to generate a LZ- MYPT1 isoform and deletion or mutation of the Tra2 $\beta$ binding site abolished E23 splicing (exon exclusion) to produce a LZ+ MYPT1. These investigators also showed that an siRNA-induced decrease of Tra- $2 \beta$ decreased E23 splicing to produce an increase in LZ+ MYPT1 expression, which is consistent with Tra- $2 \beta$ expression regulating E23 splicing and LZ+/- MYPT1 expression (Shukla and Fisher, 2008). Further investigation of the signaling pathway for the regulation of LZ+/- MYPT1 isoform expression could reveal novel targets in this pathway. Small molecules could be designed to modulate either total MYPT1 or LZ+ MYPT1 expression, which would both improve both the vascular response to endogenous $\mathrm{NO}$ and pharmacological response during the treatment of hypertension as well as a number of other diseases of the vasculature.

\section{Implications for Disease and Treatment}

1. Pressurized Resistance Vessels, Implications of the Myogenic Response for Hypertension, and Critical Analysis of Inhibitors. In most systems, flow and pressure are linearly related; as pressure increases, so does flow. However, small arteries vasoconstrict in response to an increase in pressure and vasodilate in response to a decrease in pressure, and the change in vessel diameter in response to changes in intravascular pressure is referred to as the myogenic response (Bayliss, 1902; Lassen, 1959; Davis and Hill, 1999). The myogenic response is an intrinsic property of the small resistance vessels and does not require flow (Davis and Hill, 1999). Furthermore, because of the intrinsic myogenic response as well as the modulatory actions of vasoactive substances (Bayliss, 1902; Lassen, 1959; Davis and Hill, 1999; Walsh and Cole, 2013), the resistance vessels maintain a constant blood flow over a wide range of perfusion pressures, and this property is termed the autoregulation of blood flow (Bayliss, 1902; Lassen, 1959; Davis and Hill, 1999).

Hypertension is associated with abnormalities of the myogenic response (Immink et al., 2004; Jarajapu and Knot, 2005; Kim et al., 2008b), and thus, the mechanism underlying the myogenic response is important in both health and disease. There are significant regional as well as vessel dimension differences in the magnitude and mechanism governing the autoregulation of blood flow (Jones et al., 1995; Davis and Hill, 1999). Further complicating investigation of the mechanism(s) responsible for the myogenic response are the small size $(<200$ $\mu \mathrm{m}$ diameter) of the resistance vessels; until recently, investigation of the signaling pathways regulating the myogenic response of the small resistance arteries was not possible. But, recent improvements in the sensitivity of protein phosphorylation levels have allowed for direct investigation of signaling pathways that regulate 
the myogenic response (Takeya et al., 2008; Johnson et al., 2009; El-Yazbi et al., 2010; Moreno-Dominguez et al., 2013).

Although the signaling pathway for the myogenic response is still being investigated, the first element is the mechanosensor that responds to the changes in intraluminal pressure. Martinez-Lemus et al. demonstrated that blocking integrin function with either antiintegrin antibodies (Martinez-Lemus et al., 2005) or integrin-specific peptides (Martinez-Lemus et al., 2003) results in a significant inhibition of the myogenic response. Furthermore, both the activation of integrins and the myogenic response are associated with tyrosine phosphorylation and the activation of focal adhesion kinase (FAK) and Src family tyrosine kinases (Murphy et al., 2001; 2002). Additionally, agonist stimulation of smooth muscle lead to a tyrosine phosphorylation of the protein paxillin (Pavalko et al., 1995) as well as activation of p42/44 MAPK (Spurrell et al., 2003) and the L-type $\mathrm{Ca}^{2+}$ channel (Chan et al., 2010). These data suggest that because of their ability to link the extracellular matrix to the cytoskeleton of the smooth muscle cell, integrins participate in the sensing and transmission of changes in intravascular pressure.

There are a number of studies that have demonstrated an important role for TRP channels Earley and Brayden (2015), specifically TRPC6 and TRPM4 in the myogenic response. Gonzales et al. (2014) demonstrated that selective inhibition of TRPM4 decreased the transient inward cation current induced by membrane stretch. These investigators demonstrated that the generation of $\mathrm{IP}_{3}$ by PLC $\gamma 1$ and the subsequent $\mathrm{Ca}^{2+}$ release from internal stores is required for both TRPM4 activity and myogenic tone. Furthermore, both TRPC6 inhibitors and antibodies that bind to an extracellular epitope of TRPC6, which block TRPC6 currents, attenuated the stretch-induced activation of TRPM4 current. These investigators also demonstrated that the inhibition of Src nonreceptor tyrosine kinases, which signal through PLC $\gamma$, decreased myogenic tone. These results suggest that Src tyrosine kinase activity is important in the stretch-induced increases PLC $\gamma$, which generates $\mathrm{IP}_{3}$. Subsequently, $\mathrm{IP}_{3}$ binds to the $\mathrm{IP}_{3}$ receptor, which stimulates $\mathrm{Ca}^{2+}$ release from the $\mathrm{SR}$. Thus, in response to stretch, a local increase in $\mathrm{Ca}^{2+}$ generated by both TRPC6 and $\mathrm{IP}_{3}$ are important for the activation of TRPM4, which changes membrane potential and increases the conductance of voltage-dependent $\mathrm{Ca}^{2+}$ channels, which results in smooth muscle cell contraction to generate the myogenic response.

Additionally, both $\mathrm{Ca}^{2+}$ release and $\mathrm{Ca}^{2+}$ sensitization of the contractile filaments contribute to the myogenic response. The pressure-induced increase in wall tension leads to depolarization of the smooth muscle cells, which results in opening of voltage-gated $\mathrm{Ca}^{2+}$ channels (Knot and Nelson, 1998; Davis and Hill, 1999) and an increase in intracellular $\mathrm{Ca}^{2+}$. However, in addition to the pressure-induced increase in $\mathrm{Ca}^{2+}$, other mechanisms contribute to the myogenic response (Worley et al., 1991; Osol et al., 2002). Using a highly sensitive biochemical technique, El-Yazbi et al. (2010) demonstrated that only in the presence of myogenic tone, serotonin stimulation produced a Rho kinasemediated phosphorylation of MYPT1, which induced a $\mathrm{Ca}^{2+}$ sensitization of the contractile filaments. Furthermore, this group has also demonstrated that both a Rho kinase-mediated phosphorylation of MYPT1, as well as a Rho kinase- and PKC-mediated increase in actin polymerization are important determinants of the myogenic response (Moreno-Dominguez et al., 2013; El-Yazbi et al., 2015). The activation of the $\mathrm{Ca}^{2+}$ dependent tyrosine kinase, Pyk2, has also been demonstrated to occur with $\mathrm{KCl}$ depolarization, and although the inhibition of Pyk2 did not change the rapid increase in force and RLC phosphorylation in response to $\mathrm{KCl}$ depolarization, Pyk2 inhibition did depress RLC phosphorylation, Thr-696, and Thr-850 MYPT1 phosphorylation and force during the sustained phase of the contraction (Mills et al., 2015). These results suggest that a $\mathrm{Ca}^{2+}$-induced activation of Pyk2 leads to an activation of RhoA/Rho kinase and $\mathrm{Ca}^{2+}$ sensitization, which is important for force maintenance, or the tonic phase of smooth muscle contraction.

Because hypertension is associated with abnormalities of the myogenic response (Immink et al., 2004; Jarajapu and Knot, 2005; Kim et al., 2008b), antihypertensive agents aimed at the signaling pathway for the myogenic response should be effective for the control of blood pressure. Drugs that decrease $\mathrm{Ca}^{2+}$ influx as well as agents that decrease and/or block the activation of Rho kinase signaling such as ACE inhibitors, ARBs, Rho kinase inhibitors should be and are effective antihypertensives. However, novel agents designed to decrease the sensitivity of the mechanosensor such as blocking the activation of integrins or decreasing tyrosine kinase activation could reduce blood pressure. However, tyrosine kinase inhibitors used for the treatment of carcinomas are known to be cardiotoxic (Chu et al., 2007; Force et al., 2007), and hypertension is the most common cardiovascular side effect (Chu et al., 2007). The mechanism by which tyrosine kinase inhibition produces hypertension is not well understood, but hypothesized to be due to fluid retention, endothelial dysfunction, and an inhibition of NO (Cabanillas et al., 2011). These data demonstrate the complex interplay between the myogenic response, circulating vasoactive substances, and the kidney in the regulation of blood pressure.

2. Smooth Muscle Myosin versus Nonmuscle Myosin, Implications for Force Maintenance and Vascular Tone. There are three classes of nonmuscle (NM) myosin (Golomb et al., 2004), NMIIA, NMIIB, and NMIIC, and both NMIIA and NMIIB are expressed in smooth muscle (Gaylinn et al., 1989; Morano et al., 2000; 
Lofgren et al., 2003; Eddinger et al., 2007; Yuen et al., 2009; Guvenc et al., 2010; El-Yazbi et al., 2015), whereas NMIIC is only expressed in neuronal tissue (Golomb et al., 2004; Jana et al., 2009). Both NMIIA and NMIIB are able to form bipolar thick filaments (Billington et al., 2013), and similar to SM, myosin RLC phosphorylation promotes filament formation (Ikebe and Hartshorne, 1985; Applegate and Pardee, 1992). In smooth muscle, NM myosin expression represents $\sim 10-15 \%$ of total myosin (Yuen et al., 2009; Konik et al., 2013). Furthermore, NMIIA has been demonstrated to form mixed bipolar filaments with myosin 18A (Billington et al., 2015), and SM1 and SM2 will copolymerize to form filaments (Rovner et al., 2002). However, it is unclear if NM myosin and SM myosin copolymerize to form mixed filaments or whether two distinct pools of SM and NM myosin thick filaments exist within the smooth muscle cells.

The kinetics of the individual steps of the AMATPase of NMIIB (Wang et al., 2003) and NMIIA (Kovacs et al., 2003) are slower than other types of class II myosin. NMIIA and NMIIB have a high ADP affinity, and for NMIIB, the rate of ADP release is similar to that of the steady-state ATPase. Additionally for NMIIB, actin augments $\mathrm{ADP}$ binding rather than accelerating ADP release (Wang et al., 2003), which results in NMIIB spending the majority of its kinetic cycle in states that are strongly bound to actin (Rosenfeld et al., 2003; Wang et al., 2003). On the other hand, the rate of ADP release for NMIIA is one order of magnitude faster than the ATPase, which results in NMIIA spending much less of its ATPase cycle in strongly actin bound states (Kovacs et al., 2003). Although the slow kinetics of NMIIA suggest that NMIIA could contribute to force maintenance, the high duty ratio make NMIIB an ideal candidate for the so called "latch crossbridge" (Dillon et al., 1981; Dillon and Murphy, 1982; Hai and Murphy, 1989), and NM myosin may be an important component for the sustained phase of smooth muscle contraction.

Kovacs et al. (2007) recently extended his kinetic studies and demonstrated that ADP release from NMIIB is slow and strain dependent; positive strain increases the rate of ADP release by a factor of fourfold, whereas negative strain decreases ADP release by 12 fold. Load dependence of ADP release prevents NMIIB from slowing either shortening or the rate of force generation by smooth muscle myosin. However for NMIIB, negative strain increases the duty ratio, which would contribute to force maintenance (Kovacs et al., 2007); i.e., for NMIIB, rapid ADP binding and loaddependent ADP release prolongs the attachment of NMIIB to actin at 10-100 $\mu \mathrm{M}$ ADP (at normal MgATP), which would decrease the rate of ATP usage to $<0.01$ ATP per head per second during force maintenance (Kovacs et al., 2007). Furthermore, during force maintenance both heads of the NMIIB would be attached to actin, which is ideal for a crossbridge that maintains tone.

Similar to NM myosin, the kinetics of SM myosin have been demonstrated to depend on load (Veigel et al., 2003). Using optical tweezers, these investigators demonstrated that the displacement of the SM crossbridge occurs in successive steps of 4 and $2 \mathrm{~nm}$. The duration of the first phase (4-nm displacement) is strain dependent, increasing by twofold with a negative strain and decreasing by twofold with positive strain. These results could suggest that the increase in attachment time of SM myosin due to the negative strain on the SM crossbridge during force maintenance could contribute to the latch state. Furthermore, recent data from optical trap experiments demonstrated that the attachment time of smooth muscle myosin to actin varies with SM RLC phosphorylation (Tanaka et al., 2008); when only one of the two heads of smooth muscle myosin is phosphorylated, the dwell time was fit with a double exponential with rates of 24 second $^{-1}$ and 1 second $^{-1}$, compared with a single rate of 29 second $^{-1}$ when both heads were phosphorylated. These investigators suggested that the long attachment time of the singly phosphorylated myosin could explain the latch state, or force maintenance, in smooth muscle. However, experiments in the optical trap are performed at low ionic strength to both promote actin-myosin interaction and increase interaction times; at physiologic conditions, rates are much faster. Tanaka et al. (2008) also demonstrated that the ATP turnover rate of singly phosphorylated myosin was $30 \%$ of that compared with doubly phosphorylated myosin, which contrasts with the results of Rovner et al. (2006) who demonstrated that the ATP turnover rate of myosin with a single phosphorylated head was over $50 \%$ of that when both heads were phosphorylated. The reason for the discrepancy between the results for the ATP turnover is unclear. However, it is likely that the single molecule mechanics of NM myosin with the RLC of one or both heads phosphorylated would be similar to smooth muscle myosin, although much slower, making NM myosin a more attractive candidate for a latch crossbridge.

The regulation of NM myosin has been studied in nonmuscle cells (Kolega, 2003), and similar to smooth muscle myosin, NM myosin is regulated by phosphorylation of its regulatory light chain (NM RLC); NM RLC phosphorylation promotes NM myosin filament assembly (Kolega, 2003) and also results in a 10-fold increase in the $V_{\max }$ of the NM myosin AMATPase (Cremo et al., 2001). In nonmuscle cells, Rho kinase phosphorylates the NM RLC at Ser19 (Kolega, 2003), and in epithelial cells, results are consistent with both MLCK and Rho kinase as important for the phosphorylation of NM RLC (Connell and Helfman, 2006). In epithelial carcinoma cell lines, Rho kinase increases the phosphorylation of the NM RLC at Ser19 phosphorylation of both NMIIA 
and NMIIB (Sandquist et al., 2006). During KCl depolarization of smooth muscle, both SM RLC and NM RLC phosphorylation increase, and the increase in RLC phosphorylation is not dependent on either Rho kinase or PKC (Yuen et al., 2009). However for angiotensin II (Ang II) activation, inhibition of either Rho kinase or PKC blunted SM RLC phosphorylation, whereas only a Rho kinase-dependent pathway regulated NM RLC phosphorylation (Yuen et al., 2009). These results demonstrate that similar to SM myosin, NM myosin is regulated in smooth muscle, and both MLCK and Rho kinase regulate the activation of NM myosin (Fig. 7).

Recent evidence from mechanical studies also suggests that NM myosin contributes to the force maintenance phase of smooth muscle tissue contraction. Morano et al. (2000) showed that bladder smooth muscle from transgenic mice lacking smooth muscle myosin still contract, albeit with a very slow tonic response, as opposed to the rapid phasic contraction (with transient peaks in force and $V_{\text {max }}$ ) characteristic of wild-type bladder smooth muscle (Morano et al., 2000; Lofgren et al., 2003). The force produced by NM myosin in KO tissues is low (Lofgren et al., 2003), which would suggest that NM myosin will not participate in the rapid phase of force activation, but rather the kinetics of NM myosin are tuned for force maintenance (Kovacs et al., 2007). Consistent with this hypothesis are results demonstrating that the inhibition of the NM AMATPase with blebbistatin reduced force maintenance (Rhee et al., 2006); for phasic smooth muscle, blebbistatin did not affect the rapid rise in force, but decreased maintained force, and for tonic smooth muscle, blebbistatin decreased force maintenance. However, although blebbistatin is thought to be specific for the inhibition of the NM myosin AMATPase (Straight et al., 2003), the specificity of blebbistatin for the NM versus SM AMATPase has been questioned (Eddinger et al., 2007). Nonetheless, consistent with a role of NM myosin for force maintenance are the results with heterozygous NMIIB KO mice; when compared with WT control mice, force maintenance is depressed by $25 \%$ in smooth muscle of heterozygous NMIIB KO (Yuen et al., 2009). Interestingly, Sward et al. (2000) demonstrated for carbachol activation of guinea pig ileum that inhibition of Rho kinase did not affect peak force but decreased force maintenance, and Rho/Rho kinase signaling regulates NM RLC phosphorylation (Yuen et al., 2009). These results are consistent with NM myosin participating in force maintenance (Morano et al., 2000; Lofgren et al., 2003; Rhee et al., 2006; Yuen et al., 2009; Guvenc et al., 2010), and the inhibition of Rho kinase would lead to a decrease in the activation of NM myosin (Yuen et al., 2009), which results in a reduction in both the force maintenance phase of smooth muscle contraction and vascular tone (SVR). It is also interesting to speculate on the contributions of other pathways, which have been demonstrated to be important contributors for changes in vascular tone for the regulation of NM myosin including tyrosine kinase (Moreno-Dominguez et al., 2013; El-Yazbi et al., 2015; Mills et al., 2015).

3. Force Maintenance/Latch and the Regulation of Vascular Tone: The Tonic versus Phasic Contractile Phenotype and Contributions to Pathogenesis of Hypertension. Smooth muscle contractile properties have been classified as phasic or tonic (Somlyo and Somlyo, 1968); after activation, for phasic smooth muscle, force rises rapidly to a peak before falling to a lower steady-state level, whereas for tonic smooth muscle, force slowly increases to a sustained steady state. Smooth muscle has also been termed "fast" and "slow" because of the differences in $V_{\text {max }}$. The molecular mechanism that governs tonic and/or phasic contractile properties has yet to be elucidated, although emerging evidence suggests that there are tonic and phasic contractile phenotypes (see Fisher, 2010). In general, the fast isoforms of the SM MHC (SMB) and ECL17 (ECL17a) are expressed in phasic smooth muscle while slow isoforms (SMA and ECL17b) expression predominates in tonic smooth muscle (Malmqvist and Arner, 1991). Similarly, the expression of NM myosin is significantly higher in tonic smooth muscle (Lofgren et al., 2003; Rhee et al., 2006). In addition, there are differences in the expression of the regulatory proteins in phasic and tonic smooth muscle. The expression of MLCK (Gong et al., 1992), MYPT1 (Woodsome et al., 2001), and the LZ- MYPT1 isoform (Dirksen et al., 2000; Khatri et al., 2001) is higher in phasic compared with tonic smooth muscle. Furthermore, in phasic versus tonic smooth muscle, the expression of CPI-17 is lower (Woodsome et al., 2001), whereas telokin expression is higher (Gallagher et al., 1991; Wu et al., 1998; Herring et al., 2001). These results could suggest that a gene program exists that governs the differential expression of contractile proteins in fast (phasic) versus slow (tonic) smooth muscle (Fisher, 2010), and the contractile phenotype regulates systemic vascular resistance.

Resistance is inversely related to the vessel radius to the forth power $\left(1 / \mathrm{r}^{4}\right)$, and thus, SVR is predominantly regulated at the level of the small resistance arteries with a diameter of $50-300 \mu \mathrm{m}$. Because of their small size, the molecular contractile phenotype of the resistance vessels have not been fully characterized, but the small resistance vessels express a mixture of fast and slow contractile proteins (Fisher, 2010) and exhibit a mixture of tonic and phasic contractile activity, which is referred to as vasomotion (Peng et al., 2001; Haddock and Hill, 2005). The molecular basis of essential hypertension is unknown, but the molecular contractile phenotype is known to be modulated during disease in both the large conduit vessels (Karim et al., 2004; Chen et al., 2006; Ararat and Brozovich, 2009), as well smaller resistance vessels (Zhang and Fisher, 2007; Han and Brozovich, 2013). In the small resistance 
vessels, an increase in the relative expression of protein isoforms associated with the tonic contractile phenotype (i.e., an increase in NM myosin expression) or decrease in LZ+ MYPT1 isoform expression would produce an increase in vascular tone and/or SVR, which would produce hypertension.

4. Autoregulation of Vascular Resistance/FlowMediated Vasodilatation and Nitric Oxide Signaling with Analysis of Current Inhibitors. Flow is governed by the simple equation, flow = pressure/resistance, and the ability of NO, or flow, to mediate changes in vascular tone is considered a fundamental property of the vasculature (Furchgott, 1999). The autoregulation of blood flow to a vascular bed maintains a constant flow over a wide range of pressures to ensure that perfusion is maintained despite either hypo- or hypertension. The mechanism governing the autoregulation of blood flow has yet to be fully elucidated but is known to be dependent on the intrinsic myogenic response as well as the modulatory actions of vasoactive substances (Lassen, 1959; Walsh and Cole, 2013), which importantly includes the vascular response to NO. NO is produced by the vascular endothelium in response to shear stress. The NO produced by the endothelium diffuses into the smooth muscle cells where it activates the soluble pool of guanylate cyclase and results in an increase in cGMP. cGMP activates protein kinase $\mathrm{G}$ (PKGI), which has a number of targets that produce smooth muscle relaxation (Lincoln, 1989; Schmidt et al., 1993; Alioua et al., 1998; Fukao et al., 1999; Lincoln et al., 2001), including myosin light chain (MLC) phosphatase (Surks et al., 1999). As perfusion pressure increases, the subsequent increase in blood flow will increase shear stress on the endothelial cells, which will stimulate NO production and a subsequent vasodilatation to decrease flow. Conversely, a decrease in perfusion pressure will decrease endothelial shear stress and NO production, and the resulting vasoconstriction will increase flow. Therefore, the NO-induced changes in vascular resistance can be viewed as part of a negative feedback loop that blunts the myogenic response; the myogenic response generates a vasoconstriction with an increase in pressure and vasodilatation with a decrease in pressure (Bayliss, 1902; Lassen, 1959; Davis and Hill, 1999; Walsh and Cole, 2013). Thus, NO production regulates vascular resistance and is essential for the normal regulation of blood flow.

The sensitivity and response of the vasculature to NO and NO-based vasodilators are well known to be heterogenous, and the molecular basis for this variable response to NO is controversial. Nonetheless, during NO signaling, activation of the MLC phosphatase requires the expression of a LZ+ MYPT1 isoform (Surks et al., 1999, 2003; Huang et al., 2004; Yuen et al., 2011, 2014) and in both health (Khatri et al., 2001; Huang et al., 2004; Payne et al., 2006) and disease (Karim et al., 2004; Payne et al., 2004; Zhang and Fisher, 2007; Lu et al., 2008; Dou et al., 2010; Han and Brozovich, 2013; Konik et al., 2013). The sensitivity of the vasculature to NO-mediated vasodilation is regulated by the relative expression of LZ+/LZ- MYPT1 isoforms; i.e., an increase and/or decrease in LZ+ MYPT1 expression will produce an increase and/or decrease in the sensitivity to NO, respectively. Nitrates and nitrate-based vasodilators are a well-known class of antihypertensive agents that will decrease blood pressure in the acute setting. However, tolerance to nitrates is a well-known phenomenon, which may limit the efficacy of nitrates for the treatment of hypertension, and a decrease in LZ+ MYPT1 expression has been demonstrated to contribute to the molecular mechanism of nitrate tolerance (Dou et al., 2010). In the treatment of heart failure, both ACE inhibitors (Chen et al., 2006; Chen and Brozovich, 2008) and ARBs (Ararat and Brozovich, 2009) have been demonstrated to maintain the normal expression of LZ+ MYPT1 expression and sensitivity to NO-mediated vasodilatation. In essential hypertension, both whether changes in LZ+ MYPT1 expression contribute to the molecular mechanism producing this disease and strategies to improve LZ+ MYPT1 expression and the sensitivity to NO-based vasodilators for the treatment of hypertension have not been investigated.

5. Mouse Models (Contractile Protein Knockout) and Implications for Hypertension. Several strains of genetically modified mice have been produced to evaluate the contribution of abnormalities in the regulation of vascular tone and/or vascular dysfunction to hypertension (Pfeifer et al., 1998; Brenner et al., 2000; Chutkow et al., 2002; Zhu et al., 2002; Tang et al., 2003; Michael et al., 2008; Wirth et al., 2008; Qiao et al., 2014), and experimental results and their implications will be discussed in this section.

$\mathrm{Ca}^{2+}$ signaling is well known to be important for the regulation of vascular tone (Arner and Pfitzer, 1999); the activation of voltage-gated $\mathrm{Ca}^{2+}$ channels increases cytoplasmic $\mathrm{Ca}^{2+}$ and results in vasoconstriction. However, the increase in $\mathrm{Ca}^{2+}$ also activates $\mathrm{Ca}^{2+}$-activated potassium channels (BK channels), and $\mathrm{Ca}^{2+}$ activation of these channels results in a membrane hyperpolarizing current that opposes vasoconstriction (Nelson et al., 1995) and $\mathrm{K}^{+}$channel openers, such as nicorandil, hyperpolarize smooth muscle to produce vasodilatation (Nelson et al., 1990). Brenner et al. (2003) deleted the $\beta 1$ subunit of the BK channel in mice and demonstrated that, compared with controls, the open probability of BK channels was 100-fold lower and the BK current in response to $\mathrm{Ca}^{2+}$ sparks was impaired in vascular smooth muscle from the $\beta 1 \mathrm{KO}$ animals. Furthermore, myography demonstrated that cerebral arteries from the $\beta 1 \mathrm{KO}$ animals mice were more constricted at all pressures, which resulted in hypertension in these animals. Additionally, BK channels have been demonstrated to increase their conductance in response to $\mathrm{NO} /$ cGMP signaling (Alioua et al., 1998). 
In smooth muscle, contractile agonists have been demonstrated to activate the G-proteins $G_{q}$ and $G_{11}$ and stimulate phospholipase $\mathrm{C}$, which leads to an increase intracellular $\mathrm{Ca}^{2+}$ and activation of MLCK to increase RLC phosphorylation and force (Somlyo and Somlyo, 2003). However, many vasoconstrictors also couple with $\mathrm{G}_{12}$ and $\mathrm{G}_{13}$ to activate Rho kinasemediated signaling, which inhibits MLC phosphatase to produce a $\mathrm{Ca}^{2+}$-independent increase in force (Somlyo and Somlyo, 2003). Wirth et al. (2008) produced mice with smooth muscle-specific ablation of $G_{q}-G_{11}$ or $\mathrm{G}_{12}-\mathrm{G}_{13}$ to investigate the relative contributions of $\mathrm{G}_{\mathrm{q}}-\mathrm{G}_{11}$ versus $\mathrm{G}_{12}-\mathrm{G}_{13}$ on vascular tone and the development of hypertension. In aortic smooth muscle isolated from the $\mathrm{G}_{\mathrm{q}}-\mathrm{G}_{11} \mathrm{KO}$ mice, the contractile response to both phenylephrine and Ang II was completely blocked, whereas the response to serotonin, endothelin, vasopressin, and the thromboxane analog U46619 was inhibited. Ablation of $\mathrm{G}_{12}-\mathrm{G}_{13}$ had no effect on the doseresponse relationship for phenylephrine or serotonin, but it reduced the steady-state force produced by Ang II, endothelin, vasopressin, and U46619. Furthermore, compared with WT mice, blood pressure was no different in the mice with ablation of $\mathrm{G}_{12}-\mathrm{G}_{13}$, but significantly lower in the $\mathrm{G}_{\mathrm{q}}-\mathrm{G}_{11} \mathrm{KO}$ mice, indicating that the normal regulation of blood pressure requires $G_{q}-G_{11}$ signaling. Additionally, ablation of either $\mathrm{G}_{\mathrm{q}}-\mathrm{G}_{11}$ or $\mathrm{G}_{12}-\mathrm{G}_{13}$ attenuated the increase in blood pressure produced by DOCA-salt treatment, which suggests that although $\mathrm{G}_{12}-\mathrm{G}_{13}$ signaling and the subsequent activation of a Rho kinase pathway leading to $\mathrm{Ca}^{2+}$ sensitization is not required for maintenance of normotension, it contributes to the development of DOCA-salt-dependent hypertension. These investigators also demonstrated that the RhoGEF protein LARG is important for the $\mathrm{G}_{12^{-}}-\mathrm{G}_{13}$-mediated activation of Rho kinase and DOCAinduced hypertension.

The importance of the NO/cGMP signaling pathways for the maintenance of a normal blood pressure has been established with several different models. In mice, the disruption eNOS has been demonstrated to eliminate ACh-mediated relaxation of aortic smooth muscle rings, and as would be predicted, the relaxation produced by sodium nitroprusside was no different in eNOS KO and WT animals. This defect in vascular reactivity resulted in hypertension in the eNOS KO mice (Huang et al., 1995). Similarly, PKGI KO mice were hypertensive compared with WT littermates (Pfeifer et al., 1998). These investigators demonstrated compared with WT, that although the response to contractile agonist was not different in aortic rings from the PKGI KO, AChand $8 \mathrm{Br}$-cGMP-mediated relaxation was significantly attenuated. Further preincubation of aortic cells with $8 \mathrm{Br}$-cGMP attenuated the $\mathrm{Ca}^{2+}$ transient in response to NE in WT aortic smooth muscle cells, but had no effect on the $\mathrm{Ca}^{2+}$ transient in the PKGI KO aortic smooth muscle cells.
Most contractile agonists activate $\mathrm{G}_{\mathrm{q}}$-coupled receptors, resulting in the activation of phospholipase $\mathrm{C}$, the generation of $\mathrm{IP}_{3}, \mathrm{Ca}^{2+}$ release, and the activation of MLCK as well as Rho/Rho kinase and a resulting inhibition of the MLC phosphatase (Somlyo and Somlyo, 1994; Davis and Hill, 1999). Mendelsohn's group (Tang et al., 2003) demonstrated that the dose-dependent increase in $\mathrm{IP}_{3}$ production by thrombin was inhibited by both $S$-nitrosocysteine and $8 \mathrm{Br}$-cGMP. Because NO and cGMP inhibit thromboxane signaling via a PKGmediated phosphorylation of the cytoplasmic tail of the thromboxane receptor (Wang et al., 1998) and RGS-2 terminates G-protein receptor signaling by accelerating the GTP hydrolysis rate by $\mathrm{G} \alpha$ subunits (Watson et al., 1996), these investigators (Tang et al., 2003) examined the function of RGS-2 in NO/cGMP signaling cascade. They demonstrated that phosphorylation of RGS-2 by PKG resulted in a translocation of RGS-2 from the cytosolic to particulate fractions and decreased $\mathrm{G} \alpha_{\mathrm{q}}$ GTPase activity. These data show that NO/cGMPmediated activation of PKG results in a phosphorylation of RGS-2, which increases the activation of $\mathrm{G}_{\mathrm{q}}$-coupled receptors. Consistent with these data are the results that show agonist-induced force production was increased and the vasodilatory response to $\mathrm{NO}$ was reduced in aortic smooth muscle from RGS-2 KO mice, and the resulting increase in vascular tone (relative vasoconstriction) was responsible for the increase in blood pressure in RGS-2 KO mice compared with WT littermates (Tang et al., 2003). The importance of the NO/cGMP signaling cascade for the regulation of vascular tone and blood pressure has also been demonstrated in three recent studies (Michael et al., 2008; Qiao et al., 2014; Reho et al., 2015). Michael et al. (2008) produced a knock-in mouse in which the initial four leucine/isoluecine residues of PKGI $\alpha$ were replaced by alanine. This PKGI $\alpha$ LZ mutant does not interact with MYPT1 (Surks and Mendelsohn, 2003), and as would be predicted, when compared with WT, aortic rings isolated from the PKGI $\alpha$ LZ mutant were less sensitive to ACh- and 8Br-cGMP-mediated relaxation, which produced hypertension. Qiao et al. (2014) produced a conditional MYPT1 KO, which were hypertensive compared with WT controls. The lack of MYPT1, which would disrupt the ability of the MLC phosphatase to target to its substrate, the RLC, resulted in an increase in the phosphorylation of the RLC as well as force in response to both $\mathrm{KCl}$ depolarization and contractile agonists. However, surprisingly, the sensitivity of relaxation produced by NO/cGMP signaling was similar in the control and MYPT1 KO mice. The lack of a decrease in the sensitivity to NO of secondary branches of the mesenteric arteries from MYPT1 KO animals could be explained if the conditional KO of MYPT1 increased RGS-2 expression or decreased NO/cGMP-mediated PKG phosphorylation of RGS-2 or the functional replacement of MYPT1 by MBS85, which is a member of 
the MYPT family that is also expressed in smooth muscle (Hartshorne et al., 2004). Reho et al. (2015) generated a conditional deletion of MYPT1 E24 to produce a LZ+ MYPT1 KI mouse. Vascular tissue isolated for the LZ+ MYPT1 mice was more sensitive to NO/cGMP-mediated relaxation, and as would be expected, the mice were hypotensive compared with controls. Of course, it must be kept in mind that NO, cGMP, and PKG also can dilate vascular smooth muscle by more than one mechanism, thus pathways not involving MYPT1 are also possible but we are not aware of appropriate animal models yet available to test those possibilities.

Mendelsohn's group has also demonstrated that NO signaling is regulated by estrogens; Zhu et al. (2002) compared the vascular responses in WT, iNOS KO, and estrogen receptor $\beta \mathrm{KO}$ mice. Estrogen was found to decrease PE-induced contraction of aortic smooth muscle in WT, but not iNOS KO mice. Furthermore, iNOS is upregulated by estrogen, as well as transfection with estrogen receptor $\beta$. In vascular rings from estrogen receptor $\beta \mathrm{KO}$ mice, compared with WT mice, the contractile response to $\mathrm{PE}$ was reduced, which could be the result of a decrease in iNOS in the estrogen receptor $\beta$ KO tissues. The sensitivity of smooth muscle relaxation to sodium nitroprusside was similar in estrogen receptor $\beta \mathrm{KO}$ and WT animals. Nonetheless, in the estrogen receptor $\beta \mathrm{KO}$ animals, the abnormalities in vascular tone produced hypertension.

The data presented in this section demonstrate the importance of the regulation of vascular tone for the regulation of blood pressure and consistently demonstrate that disruption in the NO/cGMP signaling pathway reduces the sensitivity to NO-mediated vasodilatation, and this decrease in the vascular response to NO and/or NO-based vasodilators produces hypertension.

\section{E. Summary of Contractile Phenotype and Contributions to Pathogenesis of Hypertension with Analysis of Current Therapies for Hypertension}

Diuretics reduce blood pressure by decreasing intravascular volume, which mechanistically fits with a Guytonian view for the regulation of blood pressure (Guyton, 1991), and diuretics, as a class, are useful for treating essential hypertension (ALLHAT and Coordinators for the ALLHAT Collaborative Research Group, 2002; Rosendorff et al., 2007). Given the importance of $\mathrm{Ca}^{2+}$ signaling for the regulation of vascular tone (Arner and Pfitzer, 1999; Somlyo and Somlyo, 2003), the benefit of CCB for treating humans with essential hypertension is not surprising (ALLHAT Officers and Coordinators for the ALLHAT Collaborative Research Group, 2002). Similarly, ACE inhibitors and ARBs will decrease the activation of the Ang II G-protein-coupled receptor and inhibit Ang II-stimulated increase in intracellular $\mathrm{Ca}^{2+}$ and activation of Rho kinase signaling to decrease vascular tone (Somlyo, 1997). Crowley et al. (2005) demonstrated that the KO of the AT1 receptor in only the peripheral vasculature decreases SVR and blood pressure, and thus, these agents would be expected to and have been demonstrated to be effective therapies of essential hypertension (Guyton, 1991; ALLHAT Officers and Coordinators for the ALLHAT Collaborative Research Group, 2002). In fact, drugs that block any G-protein-coupled receptor, i.e., $\alpha$-receptor blockers (Koshy et al., 1977), would be expected to decrease vascular tone and lower blood pressure. $\beta$-Blockers decrease the activation of the cardiac $\beta$-adrenergic receptor, and the subsequent decrease in cAMP and PKA signaling produce negative chronotropic and inotropic response, which decreases blood pressure (Rosendorff et al., 2007). However, in the vascular smooth muscle, an increase PKA activity produces a vasodilatation ((Nakamura et al., 2007; Wooldridge et al., 2004), and $\beta$-blocker therapy could potentially result in an increase in vascular tone, but because of the inhibition of central sympathetic output, SVR decreases with $\beta$-blocker therapy (Man in't Veld et al., 1988). Drugs designed to increase $\mathrm{K}^{+}$channel conductance produce smooth muscle hyperpolarization and a resulting vasodilatation of vascular smooth muscle (Nelson et al., 1990; Sobey, 2001; Barbato, 2005), and these agents have been demonstrated to both decrease blood pressure (Wang et al., 2005) and be effective treating angina (Group, 2002). Although NO and NO-based vasodilators will result in a decrease in vascular tone (Furchgott, 1999) and are effective for treating angina (Rosendorff et al., 2007), they have not been demonstrated to be useful in treating essential hypertension (Rosendorff et al., 2007), possibly because of the phenomenon of nitrate tolerance (Munzel et al., 2005).

Although there are a number of effective classes of antihypertensives (Rosendorff et al., 2007), when treating patients with essential hypertension, the selection of an antihypertensive agent does not consider the possible changes in the smooth muscle contractile phenotype that may contribute to the molecular mechanism for essential hypertension. This could explain the variable racial and individual response to drug classes (Cushman et al., 2000; Johnson, 2008; Gupta, 2010). Thus, a critical need for a personalized approach for the treatment of essential hypertension exists.

\section{F. Potential Novel Targets for Treatment of Essential Hypertension}

Despite the effectiveness of current antihypertensive (Rosendorff et al., 2007), many patients with essential hypertension either do not respond to one or more therapeutic agents or have a resistant hypertension that requires treatment with multiple classes of antihypertensives for adequate control of blood pressure. Other than the well-known racial and regional 
differences in response to various classes of antihypertensives (Cushman et al., 2000; Johnson, 2008; Gupta, 2010), there is no known method to determine for any individual drug class whether a patient with essential hypertension will have a therapeutic response or the magnitude of the response to therapy. Because the molecular mechanism that produces essential hypertension is unknown, the diversity in the response to treatment is not unexpected and demonstrates the importance of identifying the mechanism producing hypertension in each individual. As outlined in the preceding sections, there are a number of changes in the contractile phenotype that could contribute to hypertension, and the contribution of the contractile phenotype could be variable among patients.

NO-mediated vasodilatation is a fundamental property of the vasculature (Furchgott, 1999), and studies of transgenic mice have demonstrated the importance of NO/cGMP signaling for maintenance of normal SVR and blood pressure (Huang et al., 1995; Pfeifer et al., 1998; Brenner et al., 2000; Zhu et al., 2002; Michael et al., 2008; Qiao et al., 2014; Reho et al., 2015). However, nitrate-based vasodilators have not been demonstrated to be effective for the treatment of essential hypertension (Rosendorff et al., 2007), possibly because of nitrate tolerance (Munzel et al., 2005). A number of mechanisms contribute to this phenomenon, but recently a decrease in LZ+ MYPT1 isoform has been suggested to play a role in the molecular mechanism for nitrate tolerance (Dou et al., 2010). Because PKGinduced phosphorylation of LZ+ MYPT1 isoforms and subsequent activation of the MLC phosphatase (Yuen et al., 2011; Yuen et al., 2014) is a key component in the NO/cGMP signaling pathway leading to vasodilatation (Lincoln, 1989), increasing MYPT1 LZ+ expression should be effective for both for reversing nitrate tolerance and the treatment of essential hypertension. The regulation for $\mathrm{LZ}+/ \mathrm{LZ}$ - MYPT1 expression is unknown, but investigators have demonstrated that Tra- $2 \beta$ appears to be an important regulator for $\mathrm{LZ}+/ \mathrm{LZ}-\mathrm{MYPT} 1$ expression (Shukla and Fisher, 2008). Furthermore, both ACE-inhibitors (Chen et al., 2006) and ARBs (Ararat and Brozovich, 2009) were demonstrated to preserve both the normal LZ+ MYPT1 expression and sensitivity to NO-mediated vasodilation in heart failure, which may explain the benefit of these agents in treating heart failure (Pfeffer et al., 1992; Yusuf et al., 1992; Pitt et al., 2000; Yusuf et al., 2000, 2003; Granger et al., 2003) compared with other vasodilators (Awan et al., 1977), as well as the mortality differences for treatment of heart failure with hydralazine and isosorbide dinitrate versus enalapril in white and black patients (Carson et al., 1999). Investigation of the signaling pathway for the regulation of total MYPT1 as well as LZ+/- MYPT1 isoform expression could reveal novel targets in this pathway that would increase LZ+ MYPT1 expression. Small molecules could be designed to modulate either total MYPT1 or LZ+ MYPT1 expression in vascular smooth muscle, and therapies designed to increase LZ+ MYPT1 expression in vascular smooth muscle would improve both the vascular response to endogenous NO and pharmacological response during the treatment of hypertension. Other targets in the NO signaling pathway could also be exploited for the treatment of essential hypertension; i.e., novel activators of RGS-2 would be expected to decrease the activation of $\mathrm{G}_{\mathrm{q}}$-coupled receptors (Tang et al., 2003; Watson et al., 1996) and ultimately decrease vascular tone and blood pressure.

NM myosin could represent another novel target for drug development for the treatment of hypertension. The inhibition (Rhee et al., 2006), as well as the KO (Yuen et al., 2009) of NM myosin has been demonstrated to decrease force during the tonic phase of smooth muscle contraction, and thus inhibition of NM myosin would be expected to decrease both vascular tone and blood pressure. As outlined above, there are significant differences in the expression of contractile proteins between tonic and phasic smooth muscle (Fisher, 2010), and resistance vessels express a mixture of fast and slow contractile proteins (Fisher, 2010), as well as tonic and phasic contractile properties (Peng et al., 2001; Haddock and Hill, 2005). The contractile phenotype is known to be modulated during disease in both the large conduit vessels (Ararat and Brozovich, 2009) and smaller resistance vessels (Zhang and Fisher, 2007; Han and Brozovich, 2013), and thus essential hypertension could be due to changes in the gene program governing the contractile phenotype and a resulting change in the contractile properties of the resistance vessels from a phasic to more tonic phenotype. This could suggest that targeting the gene program to enhance the expression of phasic contractile proteins would decrease blood pressure and represent a novel therapy for essential hypertension.

\section{Cytoskeletal Regulation}

Regulation of smooth muscle function by myosin isoforms, particularly nonmuscle myosin isoforms is discussed above in section IV. Here we will discuss the cytoskeletal proteins that until recently were assumed to serve a primarily structural role in smooth muscle. There are three types of cytoskeletal proteins in this category: intermediate filaments, microtubules, and actin filaments.

\section{A. Intermediate Filaments, Dystrophin, Utrophin, and Microtubules}

Intermediate filaments have been studied relatively little in contractile smooth muscle but of note is literature that suggests that intermediate filaments may be the glue that sticks together different functional domains of the smooth muscle cellular cytoskeleton. 
This is based on anatomic studies of several types of smooth muscle (Devine and Somlyo, 1971; Ashton et al., 1975; Small et al., 1986; Siegman, 2014) showing that a population of actin filaments that are not directly contacting myosin surround the actomyosin filament bundles and insert into cytoplasmic "dense bodies." These cytoplasmic dense bodies in turn are connected to "dense bodies" at the surface of the cell (focal adhesions) by intermediate filaments. Studies on airway smooth muscle have also indicated that intermediate filaments form cable-like structures that connect dense bodies and that these structures have a functional plasticity due to mechanisms, yet to be defined, by which the cable length can be adjusted in a regulatory manner (Zhang et al., 2010). Intermediate filaments in contractile vascular smooth muscle have also been suggested to serve functional roles that are not simply structural. For example, these filaments bind CaMKII and are phosphorylated by this kinase (Marganski et al., 2005), but whether the intermediate filaments are serving a signaling scaffolding role or whether CaMKII is somehow changing the function of the intermediate filaments is not known. Similarly, in airway smooth muscle, PAK has been shown to phosphorylate intermediate filaments (Wang et al., 2006, 2007). Interestingly, antisense knockdown of vimentin in smooth muscle inhibits agonist-induced force development (Tang, 2008), but, again, the exact molecular mechanism involved is not clear.

1. Dystrophin/Utrophin. Of interest is the fact that smooth muscle contains large quantities of dystrophin and utrophin. These proteins have been little studied in smooth muscle, but in lung and vascular smooth muscle they and the dystroglycan complexes they form connect with caveolin and cavin in caveoli as well as the actin cytoskeleton (Palma-Flores et al., 2014; Sharma et al., 2014). Knockout of dystrophin in a mouse model decreases contractility of tracheal rings (Sharma et al., 2014).

With respect to microtubules, as expected, the microtubular network is sparse in nonproliferative, nondividing smooth muscle (Somlyo, 1980). They could serve a transport purpose, but we are unaware of such functions yet being demonstrated in contractile smooth muscle.

\section{B. Actin}

A far more extensive literature has developed with respect to actin in smooth muscle. The actin in contractile smooth muscle can be divided into that associated with smooth muscle myosin, generally called the thin filaments, and the nonmuscle actin cytoskeleton. The mechanisms of regulation of actomyosin have been discussed above. Here we will focus on the emerging role of the nonmuscle cytoskeleton in the regulation of vascular function.

Several groups have demonstrated that vasoconstrictors and myogenic contractions (Cipolla et al., 2002;
Rembold et al., 2007; Kim et al., 2008a, 2010; Tejani et al., 2011) regulate the structure of the actin cytoskeleton and its connection to focal adhesions (FAs) (Poythress et al., 2013; Saphirstein et al., 2013, 2015) (Pathway \#4, Fig. 3). These smooth muscle FAs appear to be essentially identical FAs in cultured cells except for their less dynamic nature (Poythress et al., 2013). Increasing evidence (Hill et al., 2001; Gunst and Zhang, 2008; Sun et al., 2008; Saphirstein et al., 2015) indicates that tension modulates the function of signaling pathways in the smooth muscle tissue/cell and that these biomechanical functions are mediated by remodeling of the smooth muscle focal adhesions and its nonmuscle actin cytoskeletal connections. It is important to emphasize that smooth muscles generally lack tendons for the transmission and summation of contractile forces generated by the muscles. In contrast, in smooth muscles the extracellular matrix (ECM) forms a sort of "intramuscular tendon" to which the integrins attach. It is one function of the focal adhesions, then, to channel, somehow, all contractile force generated by the contractile apparatus through the integrins and to the ECM. The exact mechanisms involved are now an active area of investigation in smooth muscle cells.

Moreno-Dominguez et al. (2014) have reported that a decline in G-actin content occurs in response to pressurization or agonist activation of cerebral resistance arteries, resulting in an increase in contractility in the absence of detectable myosin or actin phosphorylation that could be blocked by Rho kinase and PKC inhibitors. These results pointed to cytoskeletal remodeling contributing to the contractile responses observed. Smooth muscle actin exists as four different isoforms. Alpha smooth muscle actin and gamma smooth muscle actin are the dominant actins associated with myosin in the contractile filaments, what has been called "mini sarcomers," (Fatigati and Murphy, 1984; Kargacin et al., 1989; Herrera et al., 2005) in vascular and gastrointestinal smooth muscle, respectively. Beta nonmuscle and gamma nonmuscle actins are thought to be present in all smooth muscles (Fatigati and Murphy, 1984; Drew et al., 1991; Kim et al., 2008a). These four isoforms are separate gene products and clearly have different functions and different expression patterns, but interestingly they have highly similar protein sequences with no isoform sharing less than 93\% identity with any other isoform (Perrin and Ervasti, 2010). Kim et al. (2008a) mapped the individual actin isoforms in vascular smooth muscle during contraction in response to an alpha agonist and found that the overall $\mathrm{F} / \mathrm{G}$ ratio increases by about twofold in response to the alpha agonist phenylephrine, but only gamma nonmuscle actin displays a statistically significant increase in polymerization. Interesting, in response to a phorbol ester, 12-deoxyphorbol 13-isobutyrate 20 -acetate, only beta nonmuscle actin showed significant evidence of remodeling. 
The actin isoforms also appear to define distinct subcellular domains within the geography of the cell. It has been found that alpha smooth muscle actin is associated with contractile filaments, beta nonmuscle actin is associated with cytoplasmic dense bodies and focal adhesions and gamma nonmuscle actin describes in interesting submembranous cortical domain in vascular smooth muscle (Parker et al., 1994, 1998; Gallant et al., 2011). Truly specific gamma nonmuscle antibodies have only recently become available, but earlier studies described qualitatively similar organizations in gut smooth muscle (Furst et al., 1986; Small et al., 1986; North et al., 1994a,b).

\section{Focal Adhesion Remodeling}

The smooth muscle FA, by connecting with the nonmuscle actin and intermediate filament cytoskeleton, is thought to serve the purpose of transmitting force generated in the contractile filaments through the integrins to the extracellular matrix and the tissue as a whole. FAs in contractile smooth muscle, like those in cultured, migrating cells, are thought to undergo considerable plasticity and remodeling in response to biomechanical forces, agonists, and hormones. When this has been directly investigated in contractile VSMCs, some, but not all, proteins have been shown to dissociate from the bulk of the FA during vasoconstrictor stimulation. Zyxin and vasodilator-stimulated phosphoprotein (VASP), but notably not FAK, undergo Src-dependent endosomal processing and regulate, through processes not yet fully understood, vascular contractility and stiffness (Poythress et al., 2013). In contrast, in airway smooth muscle, FAK has been shown to be quite mobile, shuttling between FAs at the plasmalemma and the cell interior (Opazo Saez et al., 2004). This highlights differences not only in organ function but also in cellular mechanisms between different types of smooth muscle.

Zyxin and vasodilator-stimulated phosphoprotein (VASP) are FA proteins associated with regulation of actin polymerization. This is consistent with a model whereby in VSMCs, only proteins at the edge of the focal adhesion, furthest from the plasmalemma, are capable of breaking off and undergoing remodeling in smooth muscle. This model is consistent with recent nanoscale super-resolution imaging of cultured fibroblast focal adhesion function described by Kanchanawong et al. (2010). In the Kanchanawong model, their data describe three sublayers within individual FAs: 1) the "integrin signaling layer," containing integrin and its direct connections such as FAK closest to the plasmalemma; 2 ) the "force transduction layer," including vinculin and talin, which are more interior; and 3) the "actin regulatory layer," containing alpha actinin and zyxin, which is deepest into the cytoplasm.

\section{Link to Hypertension}

An interesting scenario occurs immediately after birth when there is a drastic demand for a decrease in pulmonary vascular resistance. This triggers a transition from the intrauterine pulmonary vascular requirement for only $8-10 \%$ of cardiac output, whereas the placenta performs the function of the primary site of gas exchange to the independent support of the newborn's lung function. As a result, in the first days to weeks of the newborn's life, dramatic vascular remodeling is required. If this fails it is associated with the disorder of persistent pulmonary hypertension of the newborn. Interestingly, the remodeling is not just of the cells of the vascular tissue but also a subcellular remodeling of the actin isoforms within the vascular cells with a persistence of excessive gamma actin being a marker of persistent pulmonary hypertension of the newborn (Fediuk and Dakshinamurti, 2015). Cortical gamma actin polymerization is reported to prevent nuclear translocation of the transcription factor YY1, which downregulates SM22, important for smooth muscle specific differentiation (Sotiropoulos et al., 1999).

A similar link between actin polymerization at a subcellular level within the vascular smooth muscle cell and blood pressure has been suggested by the effect of vasoconstrictor agonists to cause both actin polymerization and inward remodeling of resistance vessels associated with many forms of hypertension. Furthermore, inhibition of actin polymerization prevented the agonist-induced inward remodeling of the resistance vessels (Staiculescu et al., 2013). It is well known that increased intraluminal pressure elicits a myogenic vasoconstriction from resistance vessels and a further increase in blood pressure. This myogenic response has been associated with increased $\mathrm{Ca}^{2+}$ entry to the cell, activation of myosin light chain kinase, as well as increased activation of ROCK and protein kinase $\mathrm{C}$ pathways that regulate actin polymerization (MorenoDominguez et al., 2013).

Further investigation of the signaling pathway for the myogenic response has implicated integrins as the link between the extracellular matrix and the cytoskeleton that senses changes in pressure (Martinez-Lemus et al., 2003 , 2005). Because the activation of integrins and the myogenic response is associated with tyrosine phosphorylation and the activation of focal adhesion kinase (FAK) and Src family tyrosine kinases (Murphy et al., 2001,2002 ), blocking the activation of integrins or decreasing the activation of tyrosine kinase could be novel targets for treating essential hypertension. Although nonreceptor tyrosine kinases can phosphorylate paxillin and induce actin polymerization (Ohanian et al., 2005) and a PKC-mediated pathway will lead to the phosphorylation, or activation, of PYK2 and Src tyrosine kinases (Hodges et al., 2007; Chang et al., 2012), there is also evidence that receptor tyrosine kinases participate in the regulation of vascular tone. ephrin (EPH) kinases are the largest family of receptor tyrosine kinases, and the ligand for EPH tyrosine kinases are ephrins. Ephrins are also cell surface 
molecules that transduce signals into cells, and multiple EPHs will interact with multiple ephrins (Pasquale, 2008). Recently, the smooth muscle-specific deletion of the tyrosine kinase EPHB4 was demonstrated to result in decreased contractile responses to the agonist phenylephrine and hypotension in male, but not female, mice (Wang et al., 2015). Contrasting with these results are those demonstrating that blood pressure was unaffected by deletion of the tyrosine kinase EPHB 6 in male and female mice, but blood pressure was elevated and the contractile response to phenylephrine was enhanced in castrated male EPHB6 KO mice (Luo et al., 2012; Wu et al., 2012). These results demonstrate that there is interplay between the various members of the nonreceptor and receptor tyrosine kinase families as well as other factors that ultimately contribute to the regulation of blood pressure that may provide untapped targets for the development of novel antihypertensive therapeutics.

\section{Identifying Therapeutic Targets in Vascular Smooth Muscle through Biomechanical Studies}

\section{A. Arterial Stiffness as a Predictor of Negative Cardiovascular Events with Aging}

Increased arterial stiffness is a prominent concept in studies of cardiovascular disease (CVD), especially in the context of aging, as an independent biomarker and predictor of hypertension and atherosclerosis, which are associated with increased mortality rates and severe damage to organ systems via stroke, renal failure, and heart disease (Ross, 1993, 1999; Dustan et al., 1996; Blacher et al., 1999; Guerin et al., 2001; Laurent et al., 2001; Cruickshank et al., 2002; Mattace-Raso et al., 2006; Laurent and Boutouyrie, 2007). Given the overwhelming predominance and persistence of CVD as the leading cause of human death worldwide, there is great interest and urgency to develop a thorough understanding of arterial stiffness as a possible cause of, and thus a potential therapeutic target for preventing or treating, CVD in humans.

Assessment of arterial stiffness exists on multiple scale levels. Macroscale techniques that characterize the hemodynamics of pulsatile blood flow are available clinically in humans and more easily interpretable, whereas microscale techniques better elucidate the underlying mechanisms causative of changes in the physical properties of blood vessels (Kohn et al., 2015). In studying arterial stiffness, the predominant challenge moving forward is to establish an integrated model of increased arterial stiffness across macro- and microscopic scales that enable the development of treatments to alleviate or prevent later-onset CVD. Recent studies suggest that vascular smooth muscle represents an attractive therapeutic target for such designs.

1. Pulse Wave Velocity: The Clinical Standard. In vivo arterial stiffness can be measured representatively and noninvasively as pulse wave velocity (PWV).
This measurement of the pulse wave generated by cardiac systole is the ratio of the distance it travels along the vascular wall to the time delay between its arrivals at different points along the circulatory pathway. Most commonly, PWV is measured via ultrasound between the carotid and femoral arteries as representative of aortic stiffness. As the current "gold standard" of arterial stiffness measurements, increased PWV is strongly correlated to both aging and CVD (Mitchell et al., 2007; Willum-Hansen et al., 2006; Ben-Shlomo et al., 2014).

The validity of PWV as an in vivo approximation of aortic stiffness is described by the Moens-Korteweg equation:

$$
\mathrm{PWV}=\sqrt{E h / 2 \rho a}
$$

where $E$ is the incremental Young's modulus of the vessel, $h$ and $a$ are the thickness and radius of the aortic wall, respectively, and $\rho$ is blood density. The value of $E$ represents a material stiffness of the wall that is more precisely descriptive of its biomechanical properties. The Moens-Korteweg equation is itself dependent upon key assumptions: that the aortic wall is isotropic and homogeneous and that the aorta undergoes small isovolumetric changes in response to pulse wave propagation. Unfortunately, these assumptions do not hold, as demonstrated by previous studies (Clark and Glagov, 1985; Gosling and Budge, 2003); therefore, whereas PWV is an undoubtedly useful prognosticative tool, its accuracy as a biomechanical indicator is unclear.

2. The Importance of Ex Vivo Material Stiffness. It is appropriate here to distinguish between "functional stiffness"- - a relation between pulse pressure applied to a vessel and its resultant deformation, or strain-and "material stiffness," the normalization of functional stiffness to vessel geometry, most notably vessel diameter and wall thickness (Greenwald, 2007). Material stiffness corresponds most directly to the value of $E$ in the Moens-Korteweg relation and is the most independent representation of an intrinsic property to resist deformation in response to an applied force (O'Rourke, 1990; Gamble et al., 1994).

Therefore, PWV can more accurately be described as a standard for functional but not material stiffness. Functional stiffness is derived from material stiffness, but the opposite is not true. In understanding this subtle difference, the diverse terminology used in reference to aortic biomechanical properties may present significant difficulties, compounded by the prevalence of terms reciprocal to stiffness such as "compliance" and "distensibility," which are sometimes used interchangeably among studies in the context of independence from vessel geometry. The most concise discussion of this complicated canvas is provided by Bank and Kaiser (1998), who cite conflicting results 
attributable to imprecise terminology and summarize their own study, showing that changes to PWV in brachial arteries, in response to smooth muscle relaxation, are not necessarily predictive of any corresponding change in material stiffness.

Analysis of arterial mechanical properties is integral to understanding more broadly the epidemiologic link between arterial stiffness and negative cardiovascular outcomes. Although arterial PWV is an easily demonstrable and a clinically valuable measure of functional stiffness, there is also a strong impetus to evaluate material stiffness of the aortic wall directly to explain how different components of the aortic wall, a highly complex and layered network of interconnected cellular and extracellular elements, contribute to altered or defective mechanisms in disease models. This is most conveniently and commonly done ex vivo by subjecting arterial tissue to mechanical stretch.

\section{B. Regulation of Arterial Stiffness by Vascular Smooth Muscle}

Although numerous studies and quantitative models have highlighted the extracellular matrix as the primary effector of dramatic changes in arterial stiffness due to aging or other cardiovascular defects (Greenwald, 2007; Zulliger and Stergiopulos, 2007; Fleenor et al., 2010, 2012; Holzapfel and Ogden, 2010; Valentin et al., 2011; Wagenseil and Mecham, 2012), vascular smooth muscle cells (VSMCs) are implicated to be major regulatory factors of arterial stiffness overall.

1. Homeostatic Interactions between Cellular and Extracellular Components of the Arterial Wall. All major biologic components of the arterial wall play integral roles in maintaining normal cardiovascular function. Abnormalities in cellular mechanotransduction, which is regulated by cytoskeletal structures, transmembrane receptors, matrix proteins, and cellcell and cell-matrix adhesions, have been linked to changes in cell mechanics or sensory machinery, as well as structural alterations in extracellular matrix, which are commonly featured in a wide range of diseases (Ingber, 2003, 2006). Indeed, there is a critical codependence between medial elastin and VSMCs in maintaining structure and function in the arterial wall, with studies showing both that elastin regulates VSMC proliferation (Brooke et al., 2003) and that VSMC knockout phenotypes result in severe degradation of elastin (Fry et al., 2015). VSMCs have been implicated in recruitment of both elastin (Dobrin, 1978) and collagen (Bank and Kaiser, 1998) during their contractile response.

Physiologic factors such as aging and hypertension induce observable physical changes in the wall due to VSMC remodeling (Glagov et al., 1993; Intengan and Schiffrin, 2000). With aging in particular, vascular smooth muscle cells (VSMCs) exhibit increased stiffness (Qiu et al., 2010) and are capable of switching their phenotype from contractile to synthetic, resulting in fundamental changes to cellular function as well as wall structure, because remodeled VSMCs migrate and proliferate in concert with alterations to other wall components, most notably elastin and collagen (Greenwald, 2007; Avolio et al., 2011).

The endothelium has also become an increasingly visible nexus of aging-induced changes, with widespread effects on cellular and extracellular processes throughout the arterial wall and especially those linked to VSM function. Nitric oxide (NO) produced by endothelial cells induces vasodilation and relaxation in VSM (Russell and Watts, 2000). NO bioavailability is reduced with aging, which attenuates this benefit, increasing VSM stiffness as a result. This downstream effect may characterize a powerful positive feedback loop where the increased stiffness leads to further reduction of NO (Cannon, 1998; Avolio et al., 2011).

2. The Focal Adhesion and Actin Cytoskeleton as Regulatory Sites of Arterial Material Stiffness. Recently, attempts to quantify the constituency of total arterial stiffness attributable to VSMC activity have become more prevalent. Ex vivo studies have shown that VSMCs contribute significantly to total stiffness, potentially in conjunction with recruitment of collagen (Barra et al., 1993; Bank et al., 1996). Under optimally physiologic conditions for smooth muscle viability, up to $50 \%$ of maximal ex vivo aortic stiffness is observable only after activating VSMCs by alpha agonist and eliminating NO-mediated vasodilation (Gao et al., 2014b), quantifiable as shown in Fig. 8.

Therefore, it is of great interest to probe the cellular mechanisms that produce this effect to identify underlying origins of dysfunction and degeneration. A series of studies highlighting stimulated VSMCs have found that focal adhesions connecting the cortical cytoskeleton to the matrix play a critical role in the regulation of signaling cascades triggered by smooth muscle activation, independently of actomyosin crossbridges (Kim et al., 2008a, 2010; Min et al., 2012; Poythress et al., 2013). In particular, disruption of the FAK-Src signaling complex reduces contractility and stiffness, as well as biochemical markers of focal adhesion signaling, induced by smooth muscle activation (Saphirstein et al., 2013; Saphirstein and Morgan, 2014). Interestingly, these mechanisms are defective with aging (Gao et al., 2014b), and may not be limited to the focal adhesions, but are also processes inherent in the cortical actin cytoskeleton (Saphirstein et al., 2015). The normal plasticity of the cytoskeleton may be an important sort of "shock absorber" for the aortic wall that is lost with aging. A model for the focal adhesion and actin cytoskeleton in the VSMC as core machinery regulating arterial stiffness, which is compromised with aging, has emerged from these findings, as shown in Fig. 9. 


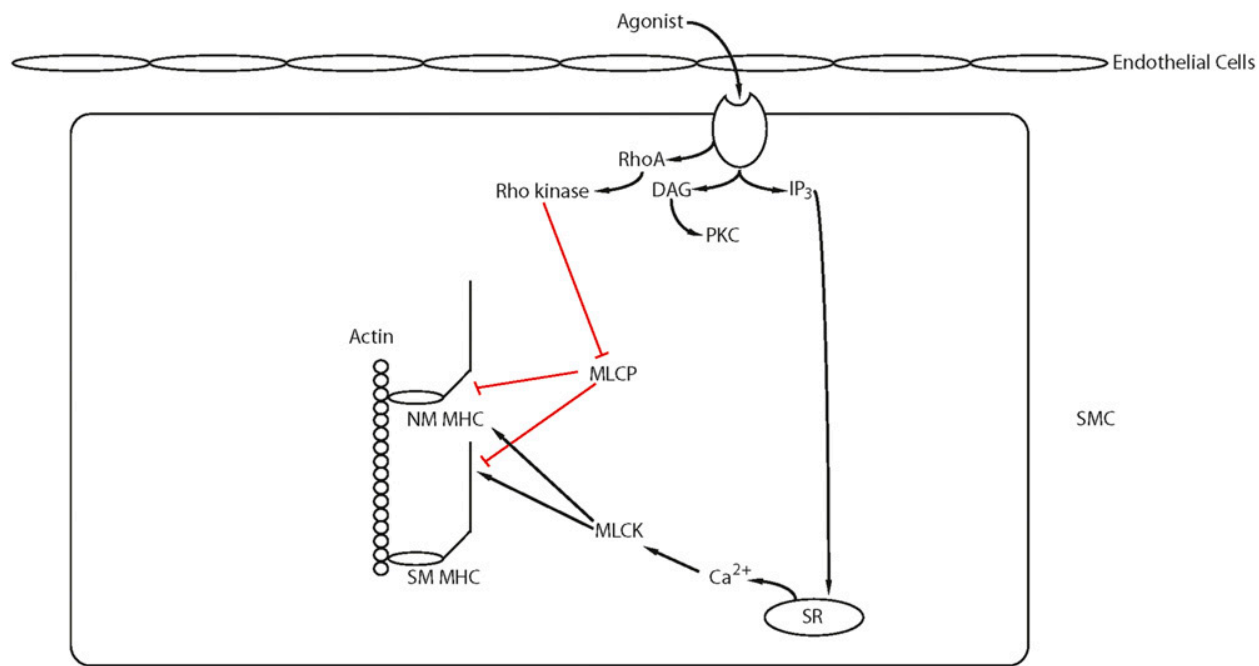

Fig. 7. Force maintenance involving either nonmuscle myosin or smooth muscle myosin: There are several proposed mechanisms for force maintenance in smooth muscle including the interaction of actin with either smooth muscle or NM myosin (latch crossbridge) as well as changes in the cytoskeleton (see text for details and see also Fig. 3).

It has become increasingly clear that VSM may play a major role in the regulation of arterial stiffness.

\section{Regulation of Vascular Smooth Muscle Cell Function by Epigenetic Mechanisms}

Epigenetic changes refer to the heritable alterations in gene expression that occur without changes in genome sequence (Jaenisch and Bird, 2003). The conventional epigenetic mechanisms include DNA methylation and histone modification, which alter the accessibility of transcription factors at DNA regulatory regions, such as promoters or enhancers (Kouzarides, 2007). Over the last decade, RNA-based modifications, which alter the translation of genetic information, have emerged as important regulators of development and disease. Collectively, these mechanisms likely play a role in the remarkable plasticity exhibited by vascular smooth muscle cells (VSMCs). This section will discuss the role of epigenetics in vascular smooth muscle (VSM) biology and describe ways by which

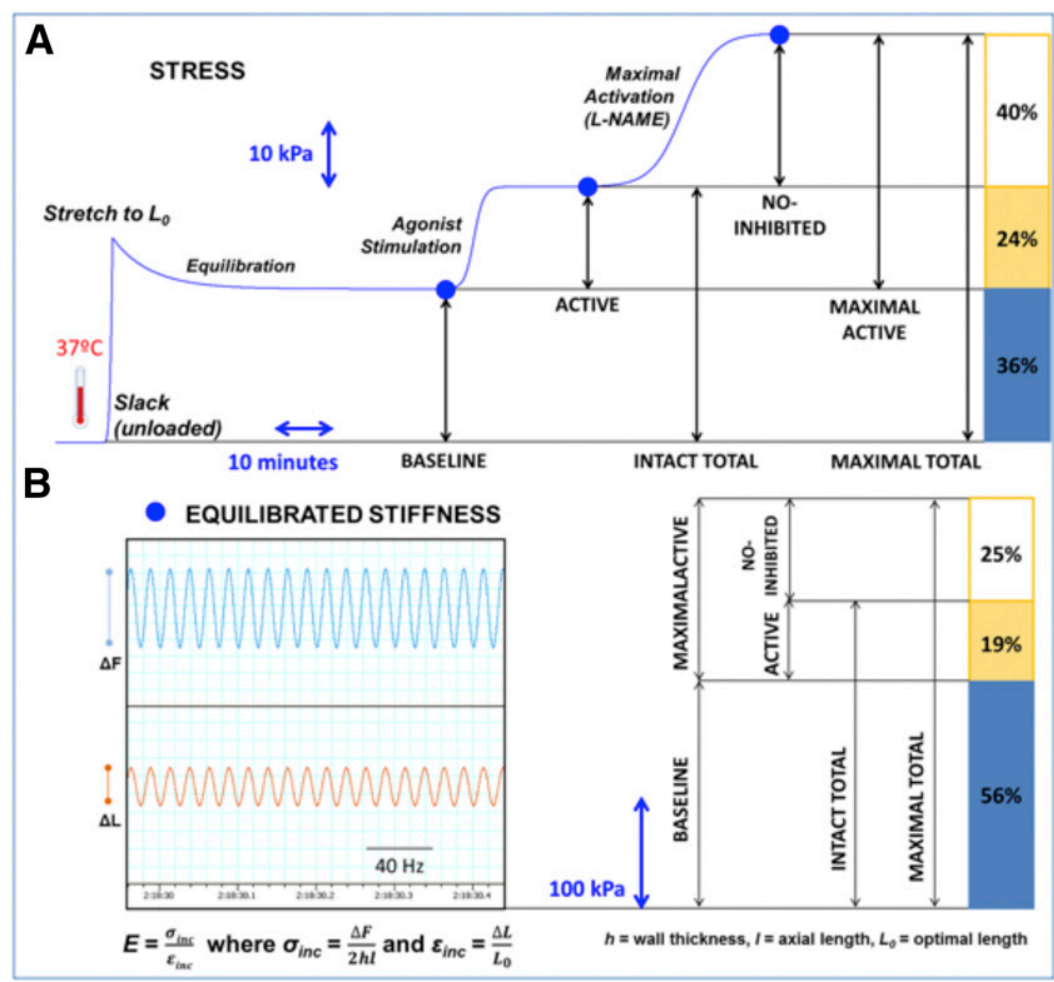

Fig. 8. Separability of contractile components to the generation of aortic stiffness. Modified from Gao et al. (2014b). 
these changes may influence the risk of cardiovascular diseases, such as hypertension.

\section{A. DNA Methylation}

DNA methylation involves the covalent addition of a methyl group to the 5 '-position of the pyrimidine ring of cytosine residues to form 5-methylcytosine (Lorenzen et al., 2012). This process, catalyzed by DNA methyltransferases, leads to transcriptional repression by blocking the access of transcription factors to response elements in the promoter region of genes. DNA methylation had long been considered irreversible until the recent discovery of the members of the ten-eleven translocation (TET 1-3) family of proteins. TET proteins oxidize 5-methylcytosine to 5-hydroxymethyl cytosine, which ultimately revert to cytosine via the demethylation pathway (Boedtkjer et al., 2011; Lorenzen et al., 2012).

Variable methylation patterns are observed in the genes of VSMCs originating from different tissues (Zhang et al., 2012). Abnormalities in DNA methylation patterns have been observed to associate with vascular diseases and have been studied extensively in atherosclerosis (Dong et al., 2002; Castro et al., 2003; Hiltunen and Yla-Herttuala, 2003). SMCs of atherosclerotic lesions in humans and animal models exhibit reduced levels of 5-methylcytosine. The resulting DNA hypomethylation enhanced switching of VSMCs from a contractile to synthetic phenotype (Laukkanen et al., 1999; Ying et al., 2000; Hiltunen et al., 2002).

\section{B. Histone Modifications}

The modifications at protruding N-terminal tails of histones act as an integral epigenetic tag for chromatin remodeling. The histone tails can be modified by acetylation, methylation, ubiquitylation, and phosphorylation (Cheung et al., 2000; Kouzarides, 2007). The unique pattern of histone modifications in SMCs can alter chromatin packaging, which leads to the differential expression of crucial VSMC genes (Alexander and Owens, 2012). For example, the chromatin structure of the CArG-box [CC(A/T)6GG] motif, essential for tissuespecific expression of SMC marker genes, exists in an easily accessible euchromatin-like form in SMCs but not in non-SMCs (Alexander and Owens, 2012). Various histone modifications, such as dimethylation of H3K4 and H3K79 and acetylation of $\mathrm{H} 4$ and $\mathrm{H} 3 \mathrm{~K} 9$, have been observed in the CArG elements of SM $\alpha$-actin and SM MHC in VSMC gene loci (McDonald et al., 2006). In contrast, these histone modifications are associated with transcriptional silencing in non-SMCs, such as endothelial cells (ECs), embryonic stem cells, and fibroblasts (McDonald et al., 2006). ECs exhibit high levels of $\mathrm{H} 4$ acetylation and H3K4 dimethylation at the EC-selective vascular endothelial cadherin gene locus. Increased levels of H4K20 dimethylation of this locus in VSMCs, however, are associated with transcriptional silencing (McDonald et al., 2006). These studies implicate histone modifications as important epigenetic tags in the regulation of SMC determination and differentiation.

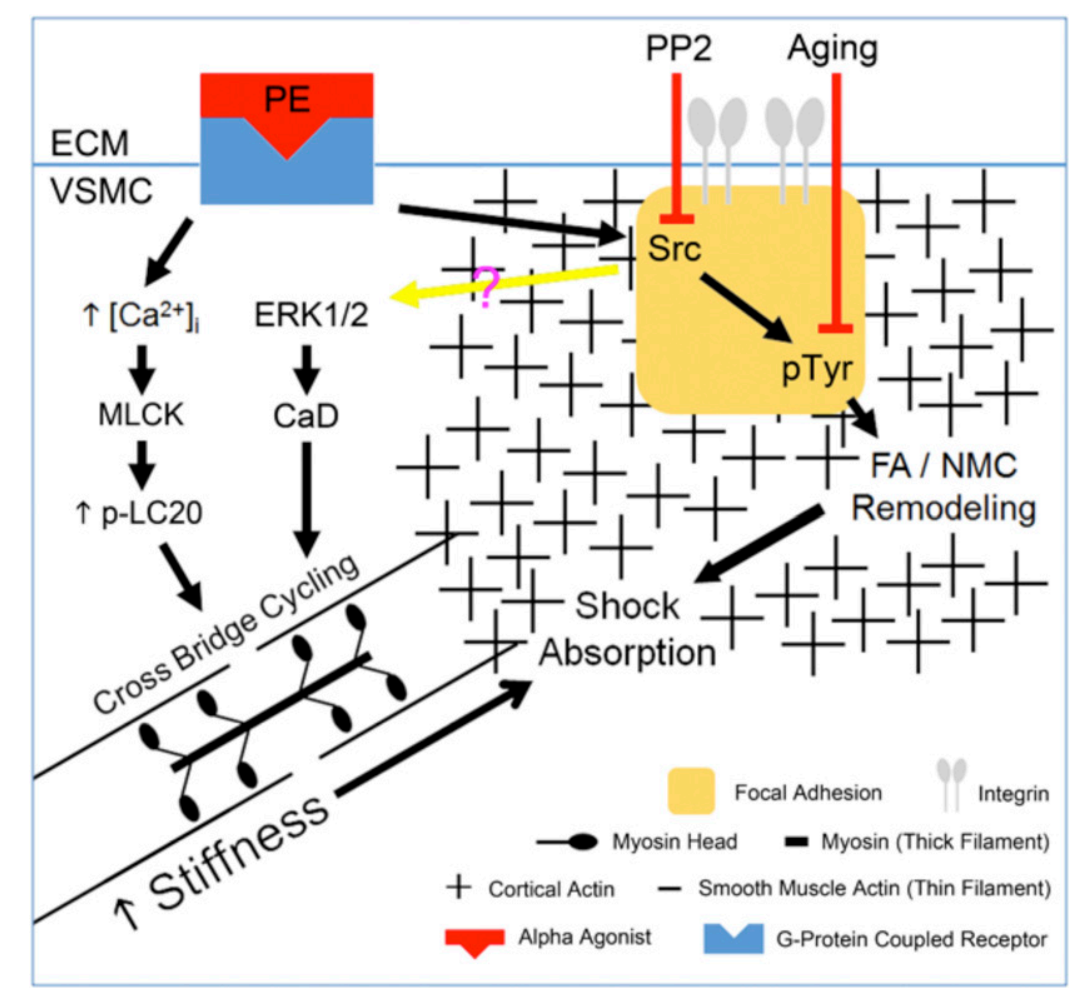

Fig. 9. A diagrammatic model of how cytoskeletal remodeling could provide plasticity and an important "shock absorber" for the cardiovascular system (modified from Gao et al., 2014b.) 
1. Histone Acetylases and Histone Deacetylases. Histone acetylation is characterized by the posttranslational modification of histones, which lead to chromatin remodeling by loosening histone-DNA contacts (Lorenzen et al., 2012). These modifications affect many SMC processes, such as differentiation, phenotypic switching, proliferation/apoptosis, and migration. Acetylation and deacetylation of histones are catalyzed by enzymes known as histone acetylases (HATs) and histone deacetylases (HDACs), respectively. HATs are classified into three families, including GNAT, MYST, and $\mathrm{CBP} / \mathrm{p} 300$ [cAMP response element-binding protein (CREB)]. HDACs are categorized into four classes in mammals: class I (HDAC1-3, HDAC8), class II (HDAC4-7, HDAC9-10), class III sirtuins (SIRT1-7), and class IV (HDAC11) (Lorenzen et al., 2012). The class I, II, and IV HDACs are $\mathrm{Zn}^{2+}$-dependent deacetylases, whereas the class III HDACs, sirtuins, possess nicotinamide adenine dinucleotide (NAD)-dependent deacetylase activity (Lorenzen et al., 2012).

HATs and HDACs work in a dynamic manner to regulate cellular gene expression. Histone acetylation by p300 HAT allows binding of serum response factor to previously inaccessible CArG-containing regions of SMC-specific genes enabling the recruitment of coactivators, such as myocardin, to stimulate expression of $\alpha$-actin (Gabbiani et al., 1981), $\gamma$-actin, calponin (Duband et al., 1993), SM22 $\alpha$ (Duband et al., 1993), h-caldesmon (Frid et al., 1992), MLC, and SM MHCs (SM1 and SM2) (Nagai et al., 1988). Retinoic acid treatment of A404 cells, an SMC embryonic cell line, enhances acetylation of histones $\mathrm{H} 3$ and $\mathrm{H} 4$ at SM $\alpha$-actin and SM MHC CArG-containing promoter regions (Manabe and Owens, 2001). Unlike myocardin, KLF4 (Kruppel-like factor 4) is a transcription factor associated with myogenic repression that regulates SMC phenotypic switching both in vitro and in vivo after vascular injury. KLF4 acts through epigenetic mechanisms to decrease histone $\mathrm{H} 4$ deacetylase by recruiting HDAC2 and HDAC5, which block the binding of serum response factor to methylated histones and CArG box chromatin during repression of SMC gene expression (McDonald and Owens, 2007; Yoshida et al., 2008).

Although the studies identifying the role of HATs and HDACs in the regulation of SMC functions warrant further in vivo studies, they highlight the relevance of inhibitors as epigenetic therapeutics in the treatment of vascular diseases. One such study demonstrated that inhibition of p300 HAT activity by Lys-CoA-TAT decreases the effects of retinoic acid on the differentiation of A404 cells toward an SMC lineage (Spin et al., 2010). In a separate study in A404 cells, overexpression of HDACs increased CREB-CArG-dependent SM22 promoter activity, whereas the HDAC inhibitor trichostatin A had the opposite effect (Qiu and Li, 2002). In contrast, HDAC inhibition after tributyrin treatment inhibited migration of cultured VSMCs after hyperacetylation of histone $\mathrm{H} 3$ and reduced expression of HDAC7 (Qiu and Li, 2002). Pharmacological inhibition of HDAC activity or knockdown of HDAC expression with small interfering RNAs (siRNA) prevents platelet-derived growth factor-induced VSMC proliferation (Findeisen et al., 2011).

Recently, HATs and HDACs have been found to act on the lysine $(\mathrm{K})$ residues of a wide range of nonhistone proteins and are therefore also denoted as KATs and KDACs, respectively (Europe-Finner et al., 2015). Nonhistone targets for KATs and KDACs include large cellular macromolecular complexes involved in the actin nucleation complex (Choudhary et al., 2009) and other proteins participating in microfilament formation and dynamics (Posern et al., 2004). A majority of proteins involved in smooth and striated muscle contraction have been shown as substrates undergoing acetylation (Karolczak-Bayatti et al., 2011; Lundby et al., 2012). KDAC8 regulates the differentiation and contraction of SMCs by interacting with $\alpha$-actin (Waltregny et al., 2005; Chen et al., 2013), tropomyosin, and cortactin proteins (Chen et al., 2013; Li et al., 2014a). KDAC6 is involved in the modulation of the cytoskeleton, cell migration, and cell-cell interactions by interacting with proteins such as SIRT2 (ValenzuelaFernandez et al., 2008). KDAC inhibition by trichostatin A and hydroxamic acid was associated with increased protein acetylation and reduction of agonist-stimulated contractions in VSM tissues (Krennhrubec et al., 2007; Karolczak-Bayatti et al., 2011; Chen et al., 2013). In a separate study, during contraction, the cytoskeletal proteins were dynamically regulated through HDAC inhibition and activation, resulting in the regulation of cytoskeletal structure (Kim et al., 2006).

a. Histone deacetylases and link to hypertension. Epigenetic mechanisms influence the changes in vascular structure that occur during hypertension (Lacolley et al., 2012). Angiotensin II (Ang II) at least partially influences vascular remodeling via HDACs in the pathogenesis of hypertension (Xu et al., 2007). Specifically, HDAC5 and HDAC4, after phosphorylation by Ang II, export from the nucleus where they activate myocyte enhancer factor-2, leading to SMC hypertrophy (Xu et al., 2007; Li et al., 2010). Treatment of spontaneously hypertensive rats with HDAC inhibitors, such as trichostatin A or valproic acid, reduced SMC hypertrophy, blood pressure, and vascular inflammation (Cardinale et al., 2010; Bogaard et al., 2011; Usui et al., 2012). All these studies highlight an important therapeutic potential for HDAC inhibitors in the treatment of vascular diseases.

2. Sirtuins. Sirtuins are a class of proteins that have multiple functions including $\mathrm{NAD}^{+}$-dependent deacetylation. Currently, there are seven known mammalian sirtuins (SIRT1-7), located in the nucleus (SIRT1, 6, and 7) (Michan and Sinclair, 2007), cytoplasm sirtuin 
(SIRT2), or mitochondria sirtuins (SIRT3, 4, and 5) (Michishita et al., 2008). Because SIRTs are multifunctional, they have a plethora of protein targets, including p53, nuclear factor $\kappa \mathrm{B}, \mathrm{Ku} 70$, FOXO, tubulin, eNOS, BAD, CytoC, Ndufa9, GDH, ACS2, and ISDH2 (Haigis and Sinclair, 2010; Corbi et al., 2013). Extensive reviews are available on the role of sirtuins in aging, calorie restriction, mitochondrial biogenesis, and neurodegenerative diseases (Gagnon et al., 2008; Pfister et al., 2008; Haigis and Sinclair, 2010; Guarente, 2011),

Sirtuins have largely been shown to have protective effects on the vasculature. For instance, SIRT1 modulates vascular biology during hypoxia-induced redox stress by deacetylating the transcription factor, HIF (Hypoxia-inducible factor)-2 $\alpha$, which modulates vascular tone and enhances cell survival through induction of antioxidant enzymes to promote angiogenesis (Dioum et al., 2009). The deacetylase activity of SIRT1 has also been shown to regulate the proliferation and migration of VSMCs via the suppression of cellular senescence mediator, p21 protein, and enhancement of senescenceresistant cell replication (Stein and Matter, 2011). Overexpression of SIRT1 increases the activity of metalloproteinase-3 inhibitor, which inhibits cell migration. In addition, SIRT1 also deacetylates and activates eNOS, which enhances NO-induced vasodilation (Mattagajasingh et al., 2007).

Interestingly, recent studies implicated SIRT1 in blood pressure regulation through targeting VSMCs. Overexpression of SIRT1 in VSMCs has been found to modulate blood pressure by downregulation of the Ang II type 1a receptor gene (Miyazaki et al., 2008). Moreover, resveratrol treatment activated SIRT1, resulting in the repression of Ang II type 1a receptor gene transcription to counteract Ang II-induced hypertension in mice (Miyazaki et al., 2008). SIRT1 expression in human VSMCs was demonstrated to correlate inversely with donor age (Thompson et al., 2014). This was associated with functional defects, such as reduced migratory capacity and increased senescence (Thompson et al., 2014). Interestingly, Ang II infusion significantly decreased the expression of SIRT1 in mouse aorta, which was associated with increased blood pressure and elevated vascular remodeling. Importantly, a VSMC-specific SIRT1 transgene attenuated both Ang II-induced increases in blood pressure and vascular remodeling (Gao et al., 2014a). These recent studies point to the potential of therapeutically improving SIRT1 function in VSMCs to treat hypertension.

\section{Noncoding RNA}

Noncoding RNAs comprise many functional RNA transcripts that are not transcribed into proteins, but regulate the transcription, stability, or translation of protein-encoding genes (Ling et al., 2013). Noncoding RNAs were once assumed only to regulate generic functions of cells, such as transcription, translation, and splicing. It is now recognized, however, that a wide variety of noncoding RNA transcripts are transcribed and have diverse biologic activity. The complex nature of this RNA-based regulatory network may partially explain the vast diversity of the characteristics of mammals, despite possessing relatively similar proteomes (Mattick and Makunin, 2006). In addition, epigenetic mechanisms, such as DNA methylation, can regulate the expression of noncoding RNAs, adding a further level of complexity (Liang et al., 2009). This section discusses microRNAs and long noncoding RNAs, which function in the broadly termed RNA silencing machinery.

1. MicroRNAs. MicroRNAs (miRs) are a family of short (21-25 nucleotide) RNAs, which typically negatively regulate protein translation by direct binding to the $3^{\prime}$ untranslated ( $3^{\prime}$ UTR) region of mRNA targets. The first miR, lin-4, was discovered to have a role in Caenorhabditis elegans larval development more than 20 years ago (Lee et al., 1993). To date, over $30,000 \mathrm{miRs}$ have been discovered in 206 species, including 2578 in humans (van Rooij and Kauppinen, 2014). It is now certain that miRs play an important role in many cellular and developmental processes. Crucially, miRs are dysregulated in many pathophysiological conditions, including cardiovascular disease.

MicroRNAs are transcribed in the nucleus by RNA polymerase II to long primary transcripts (Lee et al., 2002) (Fig. 10). They are subsequently processed by the RNAse III enzyme Drosha (Lee et al., 2003) in the nucleus to a 70 nucleotide hairpin structure termed preliminary miRNAs (pre-miRNA). After export into the cytoplasm, pre-miRNAs are further processed by a separate RNAse III enzyme, Dicer (Hutvagner et al., 2001), into a 21-25 nucleotide duplex. The mature miR strand is then incorporated onto Argonaute protein to form the RNA-induced silencing complex (RISC) (Hammond et al., 2001; Hutvagner et al., 2001), leading to unraveling and degradation of the complimentary strand (Khvorova et al., 2003; Schwarz et al., 2003). The miR then guides the RISC to complimentary sequences in the 3' UTR of target mRNA (Lee et al., 1993). In plants, the near-perfect complementarity between the miR and target sequence promotes mRNA cleavage of the target, akin to the mechanism for siRNA-induced silencing (Hake, 2003). In mammals, however, this level of complementarity is rare. In contrast, association of a 6-8 nucleotide ("seed") sequence with the 3' UTR of target mRNA (Lewis et al., 2005) leads to repression of mRNA translation through inhibition of translation initiation and/or promotion of mRNA decay (reviewed by (Ameres and Zamore, 2013)).

a. Dicer knockout mice. There are several excellent reviews that discuss the role of miRs in vascular structure, function, and disease (Yu et al., 2014; Gupta and Li, 2015; Maegdefessel et al., 2015a; Marques et al., 2015). The current review will mainly focus on miRs that have a role in regulating vascular smooth muscle 
cell (VSMC) contractility, thus influencing the risk of hypertension. The importance of miRs in vascular smooth muscle was demonstrated in experiments using smooth muscle-specific Dicer KO mice. Knockout of Dicer in vascular smooth muscle (VSM) induced embryonic lethality, which was associated with widespread hemorrhaging, loss of contractile function, and reduced VSMC proliferation (Albinsson et al., 2010). A further study using a tamoxifen-inducible SMC-specific knockout of Dicer, suggested miRs are necessary for blood pressure regulation and contractile function (Albinsson et al., 2011). Blood pressure was reduced after Dicer knockdown, with no change in cardiac dimensions. This was associated with impairment of both receptor- and calcium-mediated contraction of small mesenteric arteries and reduction of expression of contractile proteins (Albinsson et al., 2011). Furthermore, the stretchdependent increase in contractile gene expression in the portal veins of mice was dependent on Dicer expression (Turczynska et al., 2013). Loss of the miR$143 /-145$ cluster is thought to be at least partially responsible for this reduced vascular contractility (Boettger et al., 2009; Norata et al., 2012; Dahan et al., 2014). Pathways that promote the vascular smooth muscle contractile phenotype, such as Notch and bone morphogenic protein signaling, function partially through promoting the expression of miR-143/-145 (Boettger et al., 2009; Albinsson et al., 2010; Boucher et al., 2011; Davis-Dusenbery et al., 2011; Norata et al., 2012; Turczynska et al., 2013; Dahan et al., 2014). In addition to miR-143/-145, the expression of miR-21 (Kang et al., 2012) and miR-24 (Chan et al., 2010) is similarly induced by bone morphologic protein (BMP) signaling, promoting the contractile phenotype by targeting Programmed Cell Death 4 and Tribbles-like protein-3, respectively.

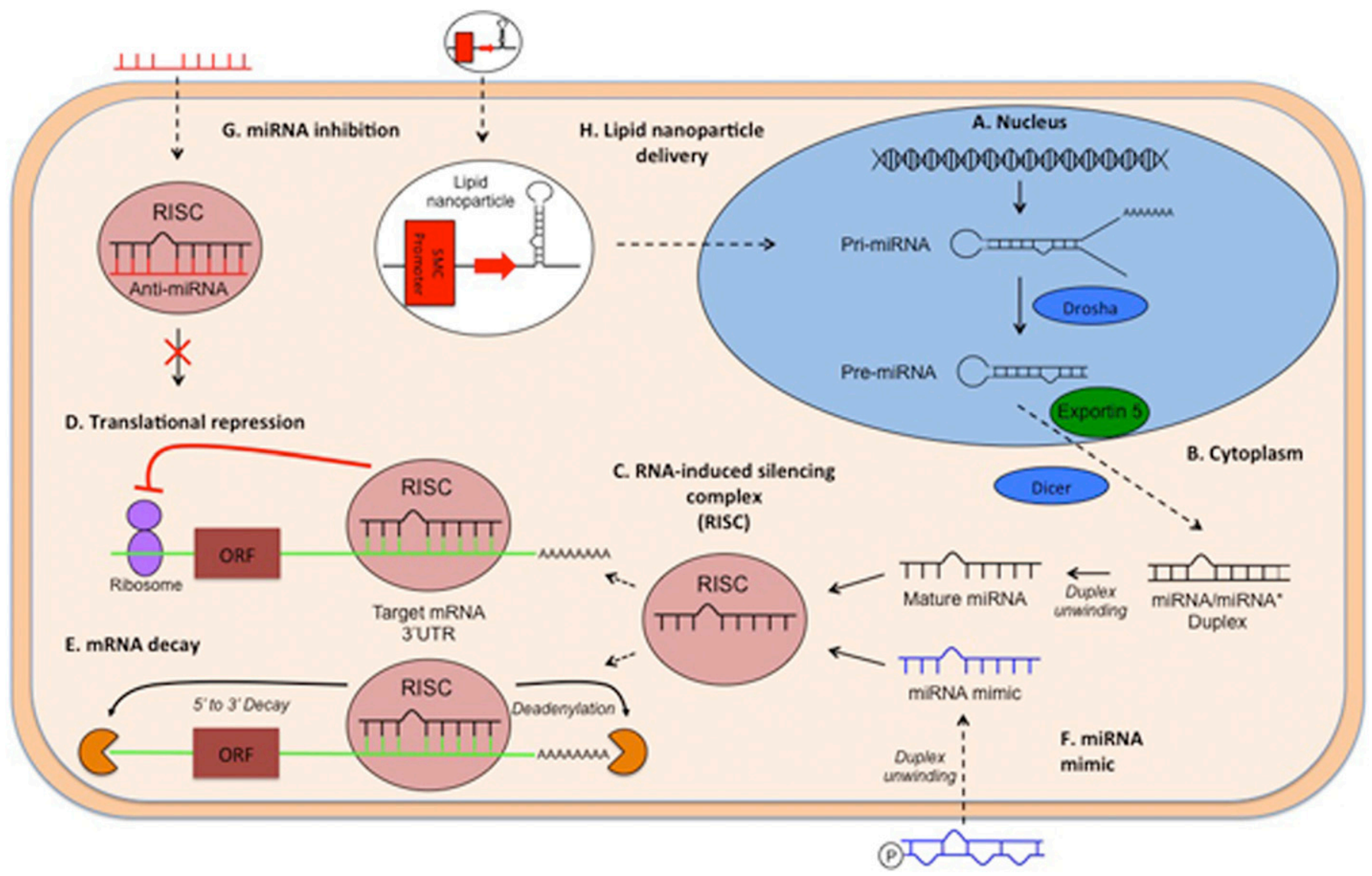

Fig. 10. Summary of the main mechanisms of miR biogenesis and function and avenues for pharmacological intervention. (A) MiRs are first transcribed in the nucleus, primarily from introns located in both coding and noncoding DNA, into long primary transcripts termed pri-miRNAs, then are processed in the nucleus by the RNAse III enzyme Drosha into a shorter ( $\sim 70$ nucleotide) hairpin duplex termed pre-miRs. (B) After export to the cytoplasm by the dsRNA-binding protein Exportin 5, pre-microRNAs are processed by an RNAse III enzyme, Dicer, into a short (21-25 nucleotide) duplex. (C) The miR duplex is unwound, primarily leading to preferential incorporation of a single strand onto Argonaute protein to form the RNAinduced silencing complex (RISC). A short sequence (6-8 nucleotides) at the $5^{\prime}$ end of the miR, known as the "seed" sequence, targets the RISC to complimentary sequences in the 3' UTR of target mRNAs. (D) Translational repression, through the blocking of translation initiation or recruitment of translational blocking proteins, and/or (E) mRNA decay, via 5' to $3^{\prime}$ decay and deadenylation of the poly (A) tail. (F) miR mimics are designed as RNA duplexes composed of a passenger strand, chemically modified (e.g., phosphate addition) to permit entry to the cell and subsequent unwinding after entry (i.e., by containing several nucleotide mismatches), and a guide strand, which consists of an identical sequence to the endogenous miR. (G) An anti-miR is designed to be complimentary to the endogenous miR of interest, thus inhibiting target mRNA binding, which therefore increases translation of the target mRNA. (H) A lipid nanoparticle delivery system can induce expression of a miR in specific cell types [e.g., in smooth muscle cells by expressing the miR under the influence of a smooth muscle cell (SMC) promoter]. 
The Dicer KO model has also demonstrated an important role for miRs in the regulation of small arterial myogenic tone, therefore modulating peripheral arterial resistance, which is raised in hypertension. Myogenic tone of mesenteric arteries was abolished from Dicer KO mice, which was associated with a loss of calcium influx through the L-type calcium channel (Bhattachariya et al., 2014). It is therefore possible that the aberrant expression of miRs may increase the development of myogenic constriction of small arteries, thereby increasing peripheral resistance and systemic blood pressure.

b. Regulation of vascular smooth muscle cell contractility. Several miRs have been found to posttranscriptionally modulate the expression of VSMC contractile proteins. Overexpression of miR-143/-145 in VSMCs increases the expression of contractile proteins, such as SM-MHC, calponin, and SM22 $\alpha$, through the targeted downregulation of KLF4 and 5 (Albinsson et al., 2010, 2011; Boucher et al., 2011; DavisDusenbery et al., 2011; Norata et al., 2012; Turczynska et al., 2013; Chettimada et al., 2014; Dahan et al., 2014; Riches et al., 2014). VSMCs from Type 2 diabetic rats (Chettimada et al., 2014) and humans (Riches et al., 2014) display an increased expression of miR-145, which contributes to the enhanced expression of vascular contractile proteins in Type 2 diabetes. Myocardin increases the expression of miR-1 in human aortic SMCs, which blocks the expression of the contractile proteins $\alpha$-SMA and SM22, possibly acting as a negative feedback mechanism to counter myocardin-induced increase in contractility (Jiang et al., 2010). Additionally, although miR-21 may promote the contractile phenotype, it may also negatively regulate VSMC contraction through the targeted downregulation of myosin phosphatase, Rho-interacting protein, and cofilin-2 (Kotlo et al., 2011).

c. Regulation of vascular smooth muscle cell ion channels. MicroRNAs have also been demonstrated to posttranscriptionally regulate the expression of VSMC ion channels, thereby influencing vascular contractility and thus blood pressure regulation. For example, miR145 indirectly increases VSM contractility by targeted downregulation of CamKII $\delta$, which results in increased expression of the $\alpha_{1 \mathrm{C}}$ subunit of the L-type calcium channel (Turczynska et al., 2012). Similarly, miR-328 has been demonstrated to directly target the $\alpha_{1 \mathrm{C}}$ subunit of the L-type calcium channel (Guo et al., 2012). Overexpression of the miR-424/322 resulted in decrease in cyclin D1 and calcium-regulating proteins calumenin and stromal-interacting molecule 1 , which reduces store-operated calcium entry in human and rat VSMCs (Merlet et al., 2013). Further studies have elucidated a role for miR-9a-3p (Li et al., 2015) and miR-190 (Li et al., $2014 \mathrm{~b})$ in the downregulation of the VSMC $\mathrm{K}_{\mathrm{ATP}}$ and potassium channel Kv7.5, respectively, causing an increase in contractility. d. Regulation of the extracellular regulated kinase pathway. MicroRNAs have also been implicated in the downregulation of proteins involved in the ERK 1/2 signaling pathway in proliferating, but not contractile, VSMCs. The miRs-21 (Stein et al., 2014), -31 (Liu et al., 2011), -132 (Choe et al., 2013), and -155 (Yang et al., 2015) all affect ERK activation. It remains to be determined if those miRs similarly affect ERK activation in fully differentiated VSMCs.

2. Long Noncoding RNAs. Long noncoding RNAs (LncRNAs), arbitrarily classified as longer than 200 nucleotides, have a role in regulating the expression of neighboring genes. LncRNAs have a much more widespread mode of action than miRs, and their function cannot currently be inferred from sequence or structure (Mercer et al., 2009; Rinn and Chang, 2012). LncRNAs can recruit chromatin-remodeling complexes to epigenetically regulate specific genomic loci. They can also influence transcriptional regulation of genes by recruiting RNA binding proteins to gene promoters, acting as cofactors to modulate transcription factor and RNA polymerase II activity. Finally, LncRNAs can also regulate posttranscriptional processing events, by recognizing complimentary sequences of RNA. Like miRs, they have the ability to bind to mRNA targets, affecting their translation and degradation, but they can also influence other posttranscriptional processes such as splicing, editing, and transport. For a deeper description of LncRNA function the reader may refer to the following references (Mercer et al., 2009; Rinn and Chang, 2012).

Although the field is newer than that for miRs, LncRNAs are emerging as potentially important players in cardiovascular disease (recently reviewed by Uchida and Dimmeler, 2015 and Miano and Long, 2015). Through RNA sequencing approaches in human coronary arteries, VSMCs have been demonstrated to express several LncRNAs, including smooth muscle and endothelial cell-enriched migration/differentiationassociated long noncoding RNA (Bell et al., 2014). Depletion of endothelial cell-enriched migration/ differentiation-associated long noncoding RNA in VSMCs was associated with decreased expression of myocardin and other contractile genes and increased expression of the promigratory proteins MDK and PTN, suggesting a role in maintenance of the contractile phenotype (Bell et al., 2014). The Lnc-Ang362 was increased in Ang II-treated rats, which was associated with an increased VSMC proliferation. Interestingly, Lnc-Ang362 was cotranscribed with miR-221 and -222 and was required for their expression (Leung et al., 2013). This study provided evidence for the regulation of LncRNAs by Ang II, which may have important implications in the pathogenesis of Ang II-associated cardiovascular diseases, such as hypertension (Leung et al., 2013).

Recent evidence suggested the expression of LncRNAs may affect VSMC proliferation and apoptosis, 
therefore influencing the risk of aortic aneurysm (He et al., 2015) and atherosclerosis (Congrains et al., 2012a; Bayoglu et al., 2014; Li et al., 2014c; Vigetti et al., 2014; Wu et al., 2014). For example, LncRNA-p21 was demonstrated to inhibit cell proliferation and neointimal hyperplasia by releasing the mouse double minute 2 repression of p53 (Wu et al., 2014). Importantly, this LncRNA was downregulated in atherosclerotic plaques from $\mathrm{ApoE}^{-1-}$ mice (Wu et al., 2014). Furthermore, the expression of the LncRNA ANRIL was influenced by several atherosclerosis-associated single nucleotide polymorphisms (SNPs) in the 9p21 locus (Holdt et al., 2011; Congrains et al., 2012a,b; Bayoglu et al., 2014). These data alert one to the possibility that previous genome-wide association studies (GWAS) may have misinterpreted SNPs in regions that encode nonprotein coding RNA as without effect on disease risk.

3. Strategies to Regulate microRNAs in Vascular Disease. MicroRNAs continue to provide great therapeutic potential, and the strategies to alter miR function will be discussed below. Because the functions of LncRNAs in VSM biology are still being determined, approaches to modify their function have not yet been extensively examined in this setting.

One of the benefits of miRs is that they are strongly conserved in species (albeit potentially targeting differing mRNA targets), thus aiding the design of preclinical studies to determine their efficacy and safety. In addition, miRs have the potential to target many members of the same molecular pathway and as a result having a greater combined effect than siRNAs, which typically target only a single gene. For example, the miR-200 family has been implicated in regulating many targets that control actin filament organization and dynamics (Bracken et al., 2014). Furthermore, miR therapy will not completely knock down their target protein's expression. Rather, they will result in a "finetuning" effect on target protein expression, making them an attractive proposition for therapeutic intervention.

There are two main techniques to modulate miR activity in vivo (reviewed in Ling et al., 2013; van Rooij and Kauppinen, 2014) (Fig. 10): 1) restoring the expression of a downregulated miR by use of a miR-mimic and 2) inhibiting the activity of an abnormally expressed miR by use of an anti-miR. A miR-mimic is a synthetically designed RNA duplex, containing a passenger strand, chemically modified to enhance uptake and disengage once inside the cell, and a guide strand, which is identical to a miR of interest. Conversely, an anti-miR is designed to be complementary to the endogenous miR and therefore binds and inhibits it from acting on its mRNA target. Several modifications, such as locked nucleic acid and phosphodiester additions, have improved the in vivo stability of anti-miRs.

The great strength of miRs, their ability to target multiple mRNAs, is also their greatest weakness because they could potentially result in many offtarget effects. In addition, miR mimics often increase the miR concentration to supraphysiologic levels, again increasing the risk of off-target actions. To lower such risk, viral constructs have been developed to re-express the miRs to endogenous levels. Recently, lipid nanoparticle delivery has proved promising to deliver miR regulators to target cells and reduced delivery to nontarget cells (Fig. 10). For instance, anti-miR-145 therapy in rats with Sugen-5416/hypoxia-induced pulmonary arterial hypertension (PAH) using the Star:Star-mPEG delivery system, reduced the severity of pulmonary hypertension without significant off-target effects (McLendon et al., 2015).

Extracellular vesicles, such as exosomes, microvesicles, and apoptotic bodies, allow long-distance delivery of cellular information, including noncoding RNA. They recently emerged as important regulators of cardiovascular diseases, such as atherosclerosis (recently reviewed by Das and Halushka, 2015) and may serve as important diagnostic and prognostic biomarkers for these conditions.

a. Pulmonary hypertension. Several miRs have been found to contribute to clinical and experimental pulmonary hypertension, which is characterized by enhanced proliferation and constriction of pulmonary artery smooth muscle cells (PASMCs). For example, miR-130a promotes PASMC proliferation through the negative regulation of its targets CDKN1A and growth arrest-specific homeobox (Wu et al., 2011; Brock et al., 2015). Interestingly, the miR-130/-301 family also regulates the expression of peroxisome proliferation receptor gamma, which influences the expression of many vasoactive factors, such as endothelin-1 (Bertero et al., 2015). The expression of miR-190 was augmented in PASMCs of the hypoxic rat, which acts as a model for pulmonary hypertension. Vasoconstriction to both potassium chloride and phenylephrine was enhanced after transfection of pulmonary arterial rings with a miR-190 mimic. The same study demonstrated that miR-190 downregulates the protein expression of the potassium channel Kv7.5, which results in raised intracellular calcium in PASMCs (Li et al., 2014b). The miR-328 has been demonstrated to directly target the $\alpha_{1 \mathrm{C}}$ subunit of the L-type calcium channel and was reduced in the pulmonary artery from patients with pulmonary hypertension, thus enabling an increase in vasoconstriction (Guo et al., 2012).

b. Systemic hypertension. There have been fewer animal studies focusing on the role of miRs in the development of essential hypertension. Clinical studies, however, have highlighted the possibility of therapeutic intervention to prevent or treat systemic hypertension. A study from Greece found that the expression of miR$145,-143$, and -133 was upregulated, and miR-21 and -1 were downregulated in the peripheral blood of patients presenting with essential hypertension (Kontaraki 
et al., 2014). These miRs are all important regulators of VSMC phenotype plasticity. A further study in a Chinese population identified the SNP rs12731181 (A to $\mathrm{G}$ ) in the prostaglandin $\mathrm{F}_{2} \alpha$ receptor that was more prevalent in hypertensive individuals (Xiao et al., 2015). This polymorphism reduces the likelihood of miR-590$3 p$ binding, thus increasing prostaglandin $\mathrm{F}_{2} \alpha$ receptor expression and enhancing prostaglandin-mediated contractility of VSMCs (Xiao et al., 2015).

c. Other vascular diseases. Several studies have demonstrated the feasibility of miR therapies to slow the development of abdominal aortic aneurysms. In particular, the miR-29 family has been heavily implicated in aneurysm development in mouse models. Murine in vivo delivery of miR-29 was found to decrease elastin mRNA and increase MMP activity and aneurysm development (Boon et al., 2011; Jones et al., 2011; Maegdefessel et al., 2012; Merk et al., 2012). Importantly, blockade of miR-29 reduced aneurysm development in Ang II-infused mice (Boon et al., 2011), a mouse model for Marfan syndrome (Merk et al., 2012), and $\mathrm{ApoE}^{-/-}$mice (Maegdefessel et al., 2012), signifying its therapeutic potential. In addition, miR-21 (Maegdefessel et al., 2012), -712/-205, and -195 (Zampetaki et al., 2014) have all been demonstrated to promote aneurysm development, whereas miR-24 (Maegdefessel et al., 2015b) slows aneurysm progression in mouse models.

Interestingly, miR-29 was found to increase with aging in the aorta from mice, which correlated with aneurysm development (Boon et al., 2011). The targets affected by miR-29 are strongly implicated in aortic stiffness, which is also known to increase with age. Few studies have focused on the role of miRs in vascular stiffness. A clinical study found that two SNPs, rs978906 (A allele) and rs9808232 (C allele), were associated with high pulse wave velocity in 856 individuals in a Chinese population (Liao et al., 2015). These polymorphisms rendered the expression of the aortic stiffness-associated ROCK2 (Noma et al., 2007) less responsive to changes in miR-1183 expression, thus enhancing ROCK2 expression (Liao et al., 2015). In addition, miR-145 was strongly reduced in $\mathrm{ApoE}^{-/-}$ mice, which was associated with an increase in collagen and stiffer arteries (Kothapalli et al., 2012).

These studies demonstrate the capacity of miR therapeutics to improve treatments for vascular complications such as hypertension, abdominal aortic aneurysm, and arterial stiffness. Indeed, anti-miR-33 (atherosclerosis, Regulus Therapeutics, (Carlsbad, CA)), anti-miR-92 (peripheral artery disease, miRagen Therapeutics Boulder, CO), and anti-miR-145 (vascular occlusion, miRagen Therapeutics) have already reached preclinical trials. This has clearly been a rapidly expanding field and we expect to see many further developments, including approaches to target LncRNAs, in the coming years.

\section{Vascular Smooth Muscle Diseases and Their Treatments}

\section{A. Review of Current Therapies and Their Targets}

Excess vasoconstriction and the resulting increase in vascular tone and SVR are an important contributing factor to the pathogenesis of essential hypertension. CCBs, ACE inhibitors, ARBs, and direct vasodilators (i.e., hydralazine) all target the smooth muscle cell to decrease vascular tone and blood pressure. CCBs decrease the activation of SM myosin by decreasing intracellular $\mathrm{Ca}^{2+}$, whereas ACE inhibitors and ARBs will decrease the activation of the RhoA/Rho kinase signaling pathway to reduce $\mathrm{Ca}^{2+}$ sensitization. $\mathrm{K}^{+}$ channel openers produce vasodilatation; nicorandil acts on BK channels and hyperpolarizes smooth muscle cells (Nelson et al., 1990), whereas minoxidil opens ATPsensitive $\mathrm{K}^{+}$channels and hyperpolarizes the smooth muscle cells (Wickenden et al., 1991). The mechanism of action for hydralazine, on the other hand, is unclear, but evidence suggests that hydralazine both activates $\mathrm{K}^{+}$ channels and also stimulates NO production by the vascular endothelium (Knowles et al., 2004).

In addition to essential hypertension, there are a number of other diseases of the vasculature and diseases with associated abnormal vascular reactivity. These defects in vascular reactivity contribute to morbidity and mortality. In this section, we will outline these diseases, their mechanism, current therapies, and suggest novel targets for both the treatment of essential hypertension as well as these other diseases of the vasculature (Table 1).

\section{B. Other Major Vascular Diseases Including Analysis of Current Therapies and Novel Targets}

1. Heart failure. Considerable evidence from both human subjects and animal models have shown that HF is associated with an impaired vasodilatory response to acetylcholine, via an endothelium-dependent mechanism, as well as to nitroglycerin, via an endothelialindependent mechanism (Kaiser et al., 1989; Kubo et al., 1991; Katz et al., 1993). Both MYPT1 LZ+ expression and the vasodilatory response to NO/cGMP has been demonstrated to decrease in HF (Karim et al., 2004; Chen et al., 2006; Ararat and Brozovich, 2009; Han and Brozovich, 2013), which demonstrates that the fall in LZ+ MYPT1 expression contributes to the decrease in sensitivity to NO-mediated vasodilatation associated with HF. Additionally, treatment of HF with either ACE inhibitors (Chen et al., 2006) or ARBs (Ararat and Brozovich, 2009) has been demonstrated to preserve the normal expression of the LZ+ MYPT1 isoform and sensitivity to NO/cGMP-mediated vasodilatation. These results are similar to those of Abassi et al., 1997; this group demonstrated that both endothelium-dependent and -independent responses in renal blood flow were impaired in HF rats compared 
TABLE 1

Disease-associated vascular abnormalities

In addition to hypertension, there are a number of diseases with abnormalities of the vasculature that contribute to patient morbidity and mortality. The vascular abnormality, possible mechanisms as well as therapies and potential new, novel targets for therapy are listed and discussed in detail in the text.

\begin{tabular}{|c|c|c|c|}
\hline Disease & Abnormality & Possible Mechanisms & Treatments \\
\hline Heart Failure & $\begin{array}{l}\text { Resting Vasoconstriction, } \\
\text { Decrease in sensitivity to NO }\end{array}$ & $\begin{array}{l}\text { Defect in NO/cGMP mediated } \\
\text { vasodilatation; ?Decrease in LZ+ } \\
\text { MYPT1 }\end{array}$ & $\begin{array}{l}\text { Vasodilators (ACE inhibitors, ARBs, } \\
\text { hydralazine, nitrates) }\end{array}$ \\
\hline $\begin{array}{l}\text { Idiopathic Pulmonary } \\
\text { Hypertension }\end{array}$ & $\begin{array}{l}\text { Pulmonary Vascular } \\
\text { Vasoconstriction \& } \\
\text { Decrease in sensitivity to NO }\end{array}$ & $\begin{array}{l}\text { Proliferation of pulmonary SMCs, } \\
\text { Defect in NO/cGMP mediated } \\
\text { vasodilatation. ?Decrease in LZ+ } \\
\text { MYPT1 and increase in NM } \\
\text { myosin }\end{array}$ & $\begin{array}{l}\text { Prostaglandins (Epoprostenol), } \\
\text { Phosphodiesterase inhibitors } \\
\text { (Sildenafil), Guanylate cyclase } \\
\text { stimulators (Riocigaut), Endothelin } \\
\text { antagonists (Bosentan), NO inhalation } \\
\text { \& Rho kinase inhibitors (Fasudil) }\end{array}$ \\
\hline Portal Hypertension & $\begin{array}{l}\text { Sensitivity to vasodilators } \\
\text { increased \& to vasoconstrictors } \\
\text { decreased }\end{array}$ & $\begin{array}{l}\text { ?Changes in isoform expression of } \\
\text { contractile proteins which } \\
\text { influence both smooth muscle } \\
\text { activation and relaxation }\end{array}$ & Management of fluid status \\
\hline Raynaud's Phenomenon & $\begin{array}{l}\text { Transient vasospasm of digital } \\
\text { vessels }\end{array}$ & $\begin{array}{l}\text { Altered reactivity of vascular } \\
\text { smooth muscle }\end{array}$ & Keeping digits warm, CCBs \\
\hline $\begin{array}{l}\text { Pre-eclampsia/Pregnancy } \\
\text { Induced Hypertension }\end{array}$ & Increase in vascular tone & $\begin{array}{l}\text { Altered reactivity to RhoA, PKC \& } \\
\mathrm{Ca}^{2+} \text {, decrease NO }\end{array}$ & Antihypertensives (non-fetotoxic) \\
\hline
\end{tabular}

with control, and Ang II receptor blockade normalized these vasodilatory responses.

The mechanism for the regulation of $\mathrm{LZ}+/ \mathrm{LZ}-$ MYPT1 expression by Ang II-induced activation of the AT1 receptor is unknown, but evidence suggests that both the activation of p42/44 MAPK (Ararat and Brozovich, 2009) and Tra2 $\beta$ (Shukla and Fisher, 2008) are important. Ang II produces vasoconstriction through its effect on both the endothelium and vascular smooth muscle (Nickenig and Harrison, 2002). Additionally, Ang II activates nuclear factor $\kappa \mathrm{B}$ to produce a proinflammatory state by inducing expression of interleukin (IL)-6 and tumor necrosis factor (TNF)- $\alpha$ (Dzau, 2001; Nickenig and Harrison, 2002) and also activates membrane $\mathrm{NADH} / \mathrm{NADPH}$ oxidases, generating reactive superoxide anions, which increase NO catabolism and therefore decrease NO bioavailability (Griendling et al., 1994). In heart failure, inhibition of Ang II signaling counter these deleterious effects and could contribute to the reduction in cardiovascular morbidity and mortality observed with ACE inhibitors and ARBs (Pfeffer et al., 1992; Yusuf et al., 1992). Furthermore, similar beneficial effects on survival have not been observed with other vasodilators such as prazosin, although prazosin has also been shown to improve cardiac index and fractional shortening for heart failure patients (Awan et al., 1977; Miller et al., 1977), which suggests that ACE inhibitor have other beneficial effects beyond reducing afterload and improving left ventricular remodeling.

In the setting of advanced heart failure, marked activation of the renin-angiotensin system and worsening functional status have been associated with a progressive elevation of the proinflammatory marker TNF- $\alpha$ (Levine et al., 1990; Torre-Amione et al., 1996), which has been demonstrated to contribute to endothelial dysfunction and also an increase in vascular tone that is produced by a RhoA/Rho kinase-mediated inhibition of MLC phosphatase (Parris et al., 1999; Mann, 2002). Additionally, the inflammatory marker IL-1 $\beta$, which is also elevated in heart failure, increases Ser850 MYPT1 phosphorylation to inhibit MLC phosphatase (Kandabashi et al., 2000; Mann, 2002). It has been demonstrated that impaired endothelial and vascular smooth muscle dysfunction are associated with a poor prognosis in heart failure (Mann, 2002). Potentially IL- $1 \beta$ and TNF- $\alpha$ could be involved in the regulation of MYPT1 isoform expression, and defining the role of inflammatory cytokines for the regulation of MYPT1 isoform expression could reveal a novel therapy to reverse the vascular dysfunction and improve prognosis in heart failure.

In addition to inhibiting the RhoA/Rho kinase pathway (Arner and Pfitzer, 1999; Somlyo and Somlyo, 2003), ACE inhibitors and ARBs also preserve the LZ+ MYPT1 isoform expression (Chen et al., 2006; Ararat and Brozovich, 2009). The variability in LZ+ MYTP1 expression among different vascular smooth muscle cells and the changes that occur during heart failure could provide an explanation as to why racial differences play an important role in predicting responses to ACE inhibitor therapy compared with the combination of vasodilators and nitrates (Carson et al., 1999). There are racial variations in plasma norepinephrine and renin (Carson et al., 1999), and this could influence both MYPT1 isoform expression as well as MLC phosphatase activity. These factors could explain the more significant blood pressure and mortality reduction seen in white compared with black patients (Carson et al., 1999). MYPT1 polymorphisms may also exist, which could contribute to the diversity of symptoms in patients with similar reductions in LVEF. Hence, the ability of ACE inhibitors and ARBs to alter the vascular smooth muscle cell phenotype could contribute to the improvement in survival in patients with heart failure (Pfeffer et al., 1992; Yusuf et al., 1992; Granger et al., 
2003), and thus enhancing LZ+ MYPT1 expression and normalizing the vascular response to NO may represent another novel target for the treatment of heart failure.

2. Pulmonary hypertension. Pulmonary arterial hypertension $(\mathrm{PAH})$ is a rare, incurable disease with a poor prognosis (Geraci et al., 2001a,b; Archer et al., 2010). In patients with PAH, the pulmonary vasculature is characterized by a resting vasoconstriction and an abnormal response to $\mathrm{NO}$, and in the majority of patients, NO-mediated vasodilatation is severely blunted (Sitbon et al., 1998; McGoon et al., 2004). Clinically, the initial assessment of patients with $\mathrm{PAH}$ includes the evaluation of the pulmonary response to NO (Badesch et al., 2004; Barst et al., 2004; McGoon et al., 2004) prognosis is improved if NO decreases pulmonary arterial pressure and PVR by $20 \%$ (Sitbon et al., 1998; Barst et al., 2004). However, less than 10\% of patients with $\mathrm{PAH}$ demonstrate a significant vasodilatory response to NO (Barst et al., 2004), and although there are a number of proposed mechanisms for the progressive increase in PVR (Stenmark et al., 2006; Archer et al., 2010; Farkas et al., 2011), the molecular mechanism that results in the lack of vasodilatation to $\mathrm{NO}$ in $\mathrm{PAH}$ is unknown.

There are two components that produce $\mathrm{PAH}$ : the extent of the structural changes (smooth muscle cell proliferation, smooth muscle cell hypertrophy, and the deposition of matrix proteins within the media of pulmonary arterial vessels (Stenmark et al., 2006; Archer et al., 2010; Farkas et al., 2011) and excess vasoconstriction (Oka et al., 2007; Archer et al., 2010). Structural changes have been documented in animal models of PAH, which have demonstrated that chronic hypoxia leads to pulmonary vascular remodeling that increases PVR and decreases the vasodilatory response to NO (reviewed in Stenmark et al., 2006; Archer et al., 2010; Farkas et al., 2011). However, despite the fact that for PAH the target and aim of all current therapeutic agents [prostaglandins (epoprostenol), phosphodiesterase inhibitors (sildenafil), guanylate cyclase stimulators (riocigaut), endothelin antagonists (bosentan), NO inhalation and Rho kinase inhibitors (fasudil)] is the smooth muscle cell to reduce pulmonary vascular resistance, the fundamental changes in the pulmonary smooth muscle contractile phenotype that contribute to the excess vasoconstriction, or the increase in PVR, as well as the decrease in vasodilatory response to NO, are poorly characterized. Evidence supports a contribution of changes in the pulmonary smooth muscle contractile phenotype in $\mathrm{PAH}$; an increase in the expression and activity of Rho kinase has been demonstrated to contribute to the pathogenesis of PAH (Connolly and Aaronson, 2011), and Rho kinase inhibition reduces pulmonary pressure in some animal models of $\mathrm{PAH}$ (Oka et al., 2007; Archer et al., 2010). The importance of the NO/cGMP signaling pathway for the pathogenesis of PAH is highlighted by data demonstrating that PKG
KO mice develop pulmonary hypertension (Zhao et al., 2012), and mice with mutations in the PKG LZ domain, which disrupts PKG interaction with MYPT1 (Surks et al., 1999, 2003; Huang et al., 2004; Yuen et al., 2011; Yuen et al., 2014), develop progressive increases in pulmonary pressures and right ventricular hypertrophy (Ramchandran et al., 2014). Furthermore, activation of guanylate cyclase has been demonstrated to improve exercise capacity and reduce pulmonary pressures in patients with PAH (Zhao et al., 2012; Ghofrani et al., 2013a,b; Ramchandran et al., 2014). All these studies are consistent with a defect in NO/cGMP signaling contributing to the pathogenesis of pulmonary hypertension, but do not isolate the step in the NO signaling pathway that produces PAH.

In smooth muscle, investigators have demonstrated that NM myosin (Fig. 7) contributes to the sustained force response (Morano et al., 2000; Lofgren et al., 2003) and a decrease in NM myosin expression will result in a decrease in vascular tone (Yuen et al., 2009). Additionally, changes in the sensitivity to NO-mediated vasodilatation are due to changes in the expression of LZ+/LZ - MYPT1 (Huang et al., 2004). Thus, an increase in NM myosin and decrease in LZ+ MYPT1 expression will produce abnormalities of pulmonary vascular reactivity and may participate in the molecular mechanism that produces PAH (Konik et al., 2013). Konik et al. (2013) demonstrated that with the development of severe PAH, there is a 3.5-fold increase in NM myosin expression, which could contribute to the prolongation in the rates of both force activation and relaxation, as well as the increase in force maintenance. Similarly, an increase in NM myosin expression has also been reported for hypoxia induced PAH (Packer et al., 1998), and therefore drugs that target NM myosin expression or activation could represent a new class of therapeutic agents for the treatment of PAH.

Konik's data also demonstrated that a decrease in LZ+ MYPT1 expression is associated with severe PAH (Konik et al., 2013). The sensitivity of smooth muscle to NO is regulated, in part, by relative LZ+ MYPT1 expression (Huang et al., 2004). In severe PAH, these data would suggest that the decrease in LZ+ MYPT1 expression would result in a decrease in the sensitivity of the pulmonary vasculature to NO. Similarly, a decrease in LZ+ MYPT1 expression has been reported for cultured pulmonary SMC exposed to hypoxia (Singh et al., 2011). Additionally, the transition from a phasic to tonic SM contractile phenotype has been suggested to be associated with a decrease in the ratio of LZ+ MYPT1/MYPT1 expression (Fisher, 2010). Tonic contractile properties would produce an increase in vascular tone, similar to that observed in patients with PAH. These results are consistent with a decrease in LZ+ MYPT1 expression contributing to the pathogenesis of $\mathrm{PAH}$; a decrease in LZ+ MYPT1 expression would reduce the sensitivity to cGMP, and riociguat 
treatment (Ghofrani et al., 2013a,b) would increase cGMP to counteract and overcome the decrease in sensitivity to NO to reduce PVR and pulmonary pressures. If a decrease in LZ+ MYPT1 expression contributes to the molecular mechanism for $\mathrm{PAH}$, these data could provide a unifying mechanism to explain the variable response to drugs that act on the NO/cGMP signaling pathway (NO, PDE5 inhibitors, and riociguat), i.e., patients with a mild decrease in LZ+ MYPT1 expression would represent "responders," whereas patients in which LZ+ MYPT1 expression is severely depressed would not respond to this class of therapeutics. Developing therapeutics that increase LZ+ MYPT1 expression should normalize the pulmonary vascular response to $\mathrm{NO}$ and decrease PVR, which would represent a novel target for treating patients with PAH.

3. Portal hypertension. Vascular reactivity is known to be altered in portal hypertension, with increased sensitivity to dilatation and decrease sensitivity to constriction (Benoit et al., 1984; Sikuler et al., 1985; Bomzon and Blendis, 1994; Wu and Benoit, 1994; Payne et al., 2004). The mechanism that contributes to this altered reactivity is unclear, but evidence suggests that splice variant expression of the contractile proteins is modulated by flow and/or pressure (Zhang and Fisher, 2007). Payne et al. (2004) examined the expression of SM MHC, ELC17, and MYPT1 in the portal vein stenosis model of portal hypertension (Benoit et al., 1984). The ligature of the portal vein produces dynamic changes in flow and pressure; there is an initial increase in both splanchnic pressure and flow (Benoit et al., 1984), which fall with the development of portosystemic shunting. Similar to these dynamic changes in flow, these investigators (Payne et al., 2004) demonstrated that in both the portal veins and mesenteric arteries within 1 day of the increase in portal pressure, although total MYPT1 expression decreased, the expression of the LZ+ MYPT1 isoform increased. These changes in MYPT1 expression were maintained for $\sim 7$ days before returning to preportal hypertension levels by 14 days after the ligature of the portal vein. Similar to MYPT1 isoform expression, ELC17 and SM MHC expression were modulated with an increase in the expression of the slow isoforms (ELC17b and SMA) 3 days after ligature, but SMA expression returned to baseline at day 14 .

Others have altered flow in the mesenteric circulation by ligating alternative pairs of second order mesenteric arteries to produce either low or high flow in the upstream first order mesenteric arteries (Zhang and Fisher, 2007). These investigators demonstrated that there was an initial increase in LZ+ MYPT1 expression in both low and high flow vessels, but by 1 month LZ+ MYPT1 expression returned toward baseline in the low flow vessels, whereas in the high flow vessels, LZ+ MYPT1 expression continued to increase. As would be predicted, the sensitivity to NO/cGMP-mediated relaxation paralleled LZ+ MYPT1 expression. Furthermore, total MYPT1 expression rapidly fell in both the low and high flow mesenteric vessels, and pretreatment with an inhibitor of the proteosome attenuated the decrease in MYPT1. These data suggest that the rapid decrease in total MYPT1 is due to degradation by the proteosome (Zhang and Fisher, 2007), which is similar to the results in the mesenteric arterioles in heart failure (Han and Brozovich, 2013). These data demonstrate that flow and/or pressure is also important in regulating the smooth muscle contractile phenotype, and thus vascular reactivity.

4. Raynaud's phenomenon. Raynaud's phenomenon is described as a transient cessation of blood flow in the fingers and toes and can be triggered by either cold or emotional stress. The phenomenon is the result of a transient vasospasm of the digital arteries in the hands and/or feet that leads to a cessation of blood flow, which produces pallor, followed by cyanosis and pain due ischemia of the sensory nerves. As the vasospastic attack subsides, blood flow to the digits is restored, and the resulting hyperemia produces a red phase. The estimates of the prevalence of this disease vary, but it is more common in women, and the onset and severity of the disease peaks between menarche and menopause. Treatment of Raynaud's involves keeping the digits warm and pharmacologic therapy with CCBs can be helpful; however the usefulness of CCBs is often limited by hypotension (Cooke and Marshall, 2005).

There are a number of mechanisms proposed for the transient vasoconstriction of the digital vessels; these have been reviewed (Cooke and Marshall, 2005) and there is evidence supporting roles for hyperactivity of adrenergic nervous system, $\alpha_{2}$ adrenoceptors, central stress responses, 5-HT, endothelin, oxidative stress, cyclo-oxygenase, and NO. However, it is interesting that the vasodilation of the digital vessels produced by both sodium nitroprusside and $\mathrm{ACh}$ are reduced in patients with Raynaud's (Morris and Shore, 1996; Cooke and Marshall, 2005), which suggest that the defect lies within the cGMP/PKG signaling cascade. Consistent with this hypothesis are the results that show L-arginine does not restore the normal vasodilatory response to $\mathrm{ACh}$ (Khan et al., 1997), which indicates that the vasodilatory response to NO is depressed in Raynaud's disease, and the mechanism lies beyond the bioavailability of NO within the cGMP/PKG signaling cascade. These data could suggest that a decrease in LZ+ MYPT1 expression in the digital vessels could contribute to the abnormal vascular reactivity.

\section{Pre-eclampsia/pregnancy-induced hypertension.} During a normal pregnancy, vascular resistance falls, which is thought to be the results of increased synthesis and activity of NO and prostacyclin (Bird et al., 2003; James and Nelson-Piercy, 2004). However, approximately $5-15 \%$ of pregnancies are complicated by hypertension, which increases perinatal morbidity and 
mortality of both the mother and fetus (Lindheimer, 1993; James and Nelson-Piercy, 2004). Antihypertensive therapy is limited due to the effects on the fetus, i.e., ACE inhibitors and ARBs are fetotoxic (James and Nelson-Piercy, 2004). A number of mechanisms have been proposed for the increase in PVR and blood pressure, including both a decrease in NO produced by endothelial dysfunction and an increase in circulating vasoconstrictors including Ang II, thromboxane, and endothelin (Khalil and Granger, 2002; Bird et al., 2003; Granger et al., 2003). NOS inhibition is commonly used to produce a model of pregnancy-induced hypertension, and studies have suggested that the increase in blood pressure is due to altered vascular reactivity produced by RhoA, $\mathrm{PKC}$, and $\mathrm{Ca}^{2+}$ signaling (Khalil and Granger, 2002; Carter and Kanagy, 2003).

$\mathrm{Lu}$ et al. (2008) recently examined MYPT1 isoform expression in pregnancy-induced hypertension. These investigators demonstrated that during a normal pregnancy total MYPT1 expression increased, but there was no change in MYPT1 isoform expression. However, during pregnancy-induced hypertension, although there were no changes in MYPT1 expression in mesenteric arteries or the aorta, in uterine arteries, pregnancyinduced hypertension was associated with an increase in LZ+ MYPT1 expression but an $\sim 50 \%$ decrease in total MYPT1 expression. As would be predicted, the increase in LZ+ MYPT1 expression was associated with an increase in the sensitivity of uterine artery relaxation to both sodium nitroprusside and cGMP. The increase in LZ+ MYPT1 expression did not occur when the blood pressure of the pregnant hypertensive rats was normalized with hydralazine. It is interesting to speculate that during pregnancy-induced hypertension the changes in MYPT1 expression are compensatory, and the resulting reduction in uterine vascular resistance maintains blood flow despite the inward remodeling of the arteries (Lu et al., 2008).

\section{Personalized Medicine}

A number of studies demonstrate that blood pressure is a genetically determined trait with heritability estimates of 31-68\% (Padmanabhan et al., 2012). There are several, rare monogenic syndromes, which are due to abnormalities in renal fluid balance that result in hypertension (Lifton et al., 2001; Ji et al., 2008). However, because the regulation of blood pressure is due to complex interactions of fluid balance, cardiac contractility, and vascular tone, the genetic basis of essential hypertension has yet to be elucidated (Oparil et al., 2003; Padmanabhan et al., 2012). Recently, a number of genome wide-association studies (GWAS) have identified SNPs in a number of genes that could contribute to the development of essential hypertension (Adeyemo et al., 2009; Johnson et al., 2011; Kato et al., 2011; Wain et al., 2011; Havulinna et al., 2013; Tragante et al., 2014). These studies have focused on populations of
European decent, but GWAS have examined East Asia (Kato et al., 2011) as well as African American cohorts (Adeyemo et al., 2009). A complete discussion of GWAS methods as well as the results of these studies are beyond the scope of the present review, and we would refer readers to recent reviews on this subject (Ehret, 2010; Padmanabhan et al., 2012). In addition to SNPs, gene expression is also regulated by miR and DNA methylation. The importance of miRs in the regulation of blood pressure is highlighted by a recent study demonstrating that mice lacking miR-142 and miR-145 have reduced vascular tone and blood pressure (Xin et al., 2009). Additionally, homv-miR-UL122 is highly expressed in hypertensive patients (Cheng et al., 2009), a number of miRs are increased in hypertensive nephrosclerosis (Wang et al., 2010), and furthermore, renin expression appears to be regulated by mi-RNA-181a and miR-663 renin (Marques et al., 2011). These factors illustrate that SNPs, miRs, and posttranslational modifications all play a role in the development of hypertension.

In addition to revealing candidate genes important in the pathogenesis of essential hypertension, pharmacogenomic GWAS have the potential to reveal the likelihood of a patient to respond to therapy or even to develop a rare adverse drug reaction (Crowley et al., 2009; Johnson et al., 2008). There are several reports of an association between polymorphisms in the $\beta_{1}$-adrenergic receptor gene and the lowering of blood pressure (reviewed in Shin and Johnson, 2007). An association of Ser49Gly and Arg389Gly polymorphisms has been demonstrated to be associated with a significant reduction in blood pressure with $\beta$-blocker therapy (Johnson et al., 2003), and furthermore, one or both of these SNPs were carried by $54 \%$ of Chinese and $44 \%$ of whites (Johnson et al., 2003), perhaps suggesting an etiology of the ethnic differences in response to $\beta$-blocker therapy. The PEAR study (Gong et al., 2012) evaluated the association of 39 SNPs known to be associated with hypertension from GWAS studies with a response to monotherapy with atenolol or HCTZ in 768 hypertensive patients (60\% white and $40 \%$ black). The response to atenolol therapy was greater in the white patients; six SNPs were associated with a response to atenolol therapy, with greater responses with in those with all six BP lowering alleles. HCTZ lowered blood pressure less in white compared with black patients. In the white patients, three alleles were associated with BP lowering for HCTZ monotherapy, and similarly, the association was strongest for those with all three alleles, but none of these were associated with BP lowering in blacks. Interestingly for HCTZ therapy, one SNP was associated with a BP reduction in white hypertensive patients, whereas it was associated with an increase in BP in the black patients. A G825C polymorphism in the gene encoding the G-protein $\beta_{3}$-subunit has been associated with a response to HCTZ therapy, and this genotype was a stronger predictor of response to 
diuretics than ethnicity (Turner et al., 2001). There have been two large trials examining polymorphisms in the genes encoding ACE (Arnett et al., 2005) and the AT1 receptor (Brunner et al., 2007) and the response to ACE inhibition. No association was found for the insertion/deletion polymorphism in the ACE gene and the response to lisinopril therapy (Arnett et al., 2005), and similarly for all ethnic groups examined, no genetic association was present for the 1166A-C genotype of the AT1 receptor and response to trandolapril. These data suggest that pharmacogenomic GWAS have the potential to identify genotypes that not only contribute to the development of hypertension but will also predict a positive response to antihypertensive therapy. An individual's genotype could be then used for a personalized approach for selecting an effective antihypertensive with minimal chance of the patient developing an adverse reaction.

\section{Summary of Novel Targets and Potential for Improved Therapies}

There is no question that essential hypertension is due, in part, to changes at the level of vascular smooth muscle that lead to excess vasoconstriction and a concomitant increase in SVR and blood pressure. In this review, we suggested have several novel targets for the treatment of essential hypertension. Details and the strategy for the each target can be found in the individual sections. These targets include those that affect $\mathrm{Ca}^{2+}$, including 1) downregulation of AKAP150, 2) increasing the expression of the $\beta 1$ subunit of the BK channel, 3) novel BK channel openers, 4) inhibiting or decreasing the expression of STIM/Orai $\left(\mathrm{Ca}^{2+}\right.$ releaseactivated $\mathrm{Ca}^{2+}$ channels), and 5) increasing cleavage of the $\mathrm{C}$ terminal of LTCC or increasing CCt expression. One could also consider inhibition of MLCK as a novel target; the soybean isoform of calmodulin (SCaM-4) has been demonstrated to bind $\mathrm{Ca}^{2+}$ but not activate MLCK and thus inhibit the activation of smooth muscle (Lee et al., 2000a; Van Lierop et al., 2002). Thus, targeted expression of SCaM-4 or a CaM fragment in vascular smooth muscle should reduce SVR and blood pressure. There are also a number of scaffolds including those for ERK (calponin, SmAV, and paxillin) and MLC phosphatase (M-RIP and Par-4) that could be examined as potential therapeutic targets, which could result in a reduction of vascular tone. Inhibition of the signaling pathways for $\mathrm{Ca}^{2+}$ sensitization including Rho kinase and GEF signaling could represent another area for rational drug design, and similarly, activation of pathways leading to $\mathrm{Ca}^{2+}$ desensitization including guanylate cyclase and increasing the expression of LZ+ MYPT1 would enhance NO/flow mediated vasodilatation, which should decrease blood pressure. Other attractive targets would be pathways leading to remodeling of the actin cytoskeleton including integrins, focal adhesion proteins, as well as the activation of tyrosine kinases. Targeted delivery of miRs to the vasculature also may prove to be an effective strategy for the treatment of hypertension; miRs could be used as a strategy to change vascular smooth muscle gene program from high force (tonic) and NO unresponsive to a lower force (phasic) and NO responsive and hence produce a reduction in vascular tone and blood pressure.

These novel targets could form the basis for rationale drug design, and could be exploited for development of therapeutic agents that are effective for the treatment of hypertension. For some targets, small molecule inhibitors/activators would appear to be a reasonable choice, whereas for other targets, i.e., to reverse the decreases in MYPT1 expression, adenoviral delivery of MYPT1 to the vasculature could also be used as a strategy to increase the sensitivity to NO or flowmediated vasodilation, which would decrease in vascular tone and blood pressure.

The contribution of alteration in the vascular phenotype from $\mathrm{Ca}^{2+}$ signaling, regulation of the cytoskeleton and contractility, and biomechanics to the pathogenesis of essential hypertension may vary among ethnic groups and/or individuals. Currently, antihypertensive therapy is generally approached by a method that could be best summarized as trial and error: an agent is selected, usually without consideration of the patient's ethnic background, and if control is not adequate, either the dose is increased or another agent is added.

In this review, we discussed the importance of the vascular phenotype in the mechanism that leads to essential hypertension. Therefore, mechanisms to identify the etiology of the increase in blood pressure in each patient are important for ultimately selecting an individualized and effective therapeutic agent. These future studies will hopefully lead to a more personalized approach to antihypertensive therapy.

\section{Authorship Contributions}

Wrote or contributed to the writing of the manuscript: Brozovich, Nicholson, Degen, Gao, Aggarwal, and Morgan.

\section{References}

Abassi ZA, Gurbanov K, Mulroney SE, Potlog C, Opgenorth TJ, Hoffman A, Haramati A, and Winaver J (1997) Impaired nitric oxide-mediated renal vasodilation in rats with experimental heart failure: role of angiotensin II. Circulation 96:3655-3664.

Adeyemo A, Gerry N, Chen G, Herbert A, Doumatey A, Huang H, Zhou J, Lashley K, Chen Y, and Christman M, et al. (2009) A genome-wide association study of hypertension and blood pressure in African Americans. PLoS Genet 5:e1000564.

Alahyan M, Webb MR, Marston SB, and El-Mezgueldi M (2006) The mechanism of smooth muscle caldesmon-tropomyosin inhibition of the elementary steps of the actomyosin ATPase. J Biol Chem 281:19433-19448.

Albert AP and Large WA (2002) A Ca2+-permeable non-selective cation channel activated by depletion of internal $\mathrm{Ca} 2+$ stores in single rabbit portal vein myocytes. $J$ Physiol 538:717-728.

Albinsson S, Skoura A, Yu J, DiLorenzo A, Fernández-Hernando C, Offermanns S, Miano JM, and Sessa WC (2011) Smooth muscle miRNAs are critical for post-natal regulation of blood pressure and vascular function. PLoS One 6:e18869.

Albinsson S, Suarez Y, Skoura A, Offermanns S, Miano JM, and Sessa WC (2010) MicroRNAs are necessary for vascular smooth muscle growth, differentiation, and function. Arterioscler Thromb Vasc Biol 30:1118-1126.

Alexander MR and Owens GK (2012) Epigenetic control of smooth muscle cell differentiation and phenotypic switching in vascular development and disease. Annu Rev Physiol 74:13-40. 
Ali F, Chin L, Pare PD, and Seow CY (2007) Mechanism of partial adaptation in airway smooth muscle after a step change in length. $J$ Appl Physiol 103:569-577. Alioua A, Tanaka Y, Wallner M, Hofmann F, Ruth P, Meera P, and Toro L (1998) The large conductance, voltage-dependent, and calcium-sensitive $\mathrm{K}+$ channel, Hslo, is a target of cGMP-dependent protein kinase phosphorylation in vivo. $\mathrm{J}$ Biol Chem 273:32950-32956.

ALLHAT and Coordinators for the ALLHAT Collaborative Research Group. The Antihypertensive and Lipid-Lowering Treatment to Prevent Heart Attack Trial (2002) Major outcomes in moderately hypercholesterolemic, hypertensive patients randomized to pravastatin vs usual care: The Antihypertensive and LipidLowering Treatment to Prevent Heart Attack Trial (ALLHAT-LLT). JAMA 288: 2998-3007.

Amberg GC, Bonev AD, Rossow CF, Nelson MT, and Santana LF (2003) Modulation of the molecular composition of large conductance, $\mathrm{Ca}(2+)$ activated $\mathrm{K}(+)$ channels in vascular smooth muscle during hypertension. $J$ Clin Invest 112:717-724.

Amberg GC and Navedo MF (2013) Calcium dynamics in vascular smooth muscle. Microcirculation 20:281-289.

Amberg GC, Navedo MF, Nieves-Cintrón M, Molkentin JD, and Santana LF (2007) Calcium sparklets regulate local and global calcium in murine arterial smooth muscle. J Physiol 579:187-201.

Amberg GC, Rossow CF, Navedo MF, and Santana LF (2004) NFATc3 regulates Kv2.1 expression in arterial smooth muscle. J Biol Chem 279:47326-47334.

Ameres SL and Zamore PD (2013) Diversifying microRNA sequence and function. Nat Rev Mol Cell Biol 14:475-488.

Appel S, Allen PG, Vetterkind S, Jin JP, and Morgan KG (2010) h3/Acidic calponin: an actin-binding protein that controls extracellular signal-regulated kinase 1/2 activity in nonmuscle cells. Mol Biol Cell 21:1409-1422.

Applegate D, Feng W, Green RS, and Taubman MB (1994) Cloning and expression of a novel acidic calponin isoform from rat aortic vascular smooth muscle. $J$ Biol Chem 269:10683-10690.

Applegate D and Pardee JD (1992) Actin-facilitated assembly of smooth muscle myosin induces formation of actomyosin fibrils. J Cell Biol 117:1223-1230.

Ararat E and Brozovich FV (2009) Losartan decreases p42/44 MAPK signaling and preserves LZ+ MYPT1 expression. PLoS One 4:e5144.

Archer SL, Weir EK, and Wilkins MR (2010) Basic science of pulmonary arterial hypertension for clinicians: new concepts and experimental therapies. Circulation 121:2045-2066.

Arner A and Pfitzer G (1999) Regulation of cross-bridge cycling by $\mathrm{Ca} 2+$ in smooth muscle. Rev Physiol Biochem Pharmacol 134:63-146.

Arnett DK, Davis BR, Ford CE, Boerwinkle E, Leiendecker-Foster C, Miller MB Black H, and Eckfeldt JH (2005) Pharmacogenetic association of the angiotensinconverting enzyme insertion/deletion polymorphism on blood pressure and cardiovascular risk in relation to antihypertensive treatment: the Genetics of Hypertension-Associated Treatment (GenHAT) study. Circulation 111:3374-3383.

Asano T, Suzuki T, Tsuchiya M, Satoh S, Ikegaki I, Shibuya M, Suzuki Y, and Hidaka $\mathrm{H}$ (1989) Vasodilator actions of HA1077 in vitro and in vivo putatively mediated by the inhibition of protein kinase. Br J Pharmacol 98:1091-1100.

Ashton FT, Somlyo AV, and Somlyo AP (1975) The contractile apparatus of vascular smooth muscle: intermediate high voltage stereo electron microscopy. $\mathrm{J} \mathrm{Mol} \mathrm{Biol}$ 98:17-29.

Aubart FC, Sassi Y, Coulombe A, Mougenot N, Vrignaud C, Leprince P, Lechat P, Lompré AM, and Hulot JS (2009) RNA interference targeting STIM1 suppresse vascular smooth muscle cell proliferation and neointima formation in the rat. Mol Ther 17:455-462.

Avolio A, Butlin M, Liu Y-Y, Viegas K, Avadhanam B, and Lindesay G (2011) Regulation of arterial stiffness: Cellular, molecular and neurogenic mechanisms. Ar tery Res 5:122-127.

Awan NA, Miller RR, DeMaria AN, Maxwell KS, Neumann A, and Mason DT (1977) Efficacy of ambulatory systemic vasodilator therapy with oral prazosin in chronic refractory heart failure. Concomitant relief of pulmonary congestion and elevation of pump output demonstrated by improvements in symptomatology, exercise tolerance, hemodynamics and echocardiography. Circulation 56:346-354.

Babij P and Periasamy M (1989) Myosin heavy chain isoform diversity in smooth muscle is produced by differential RNA processing. J Mol Biol 210:673-679.

Babu GJ, Loukianov E, Loukianova T, Pyne GJ, Huke S, Osol G, Low RB, Paul RJ, and Periasamy M (2001) Loss of SM-B myosin affects muscle shortening velocity and maximal force development. Nat Cell Biol 3:1025-1029.

Babu GJ, Pyne GJ, Zhou Y, Okwuchukuasanya C, Brayden JE, Osol G, Paul RJ, Low $\mathrm{RB}$, and Periasamy M (2004) Isoform switching from SM-B to SM-A myosin results in decreased contractility and altered expression of thin filament regulatory proteins. Am J Physiol Cell Physiol 287:C723-C729.

Badesch DB, Abman SH, Ahearn GS, Barst RJ, McCrory DC, Simonneau G, and McLaughlin VV; American College of Chest Physicians (2004) Medical therapy for pulmonary arterial hypertension: ACCP evidence-based clinical practice guidelines. Chest 126(1, Suppl)35S-62S.

Baker JE, Brosseau C, Fagnant P, and Warshaw DM (2003) The unique properties of tonic smooth muscle emerge from intrinsic as well as intermolecular behaviors of Myosin molecules. J Biol Chem 278:28533-28539.

Bank AJ and Kaiser DR (1998) Smooth muscle relaxation: effects on arterial compliance, distensibility, elastic modulus, and pulse wave velocity. Hypertension 32: 356-359.

Bank AJ, Wang H, Holte JE, Mullen K, Shammas R, and Kubo SH (1996) Contribution of collagen, elastin, and smooth muscle to in vivo human brachial artery wall stress and elastic modulus. Circulation 94:3263-3270.

Bannister JP, Leo MD, Narayanan D, Jangsangthong W, Nair A, Evanson KW, Pachuau J, Gabrick KS, Boop FA, and Jaggar JH (2013) The voltage-dependent L-type $\mathrm{Ca} 2+(\mathrm{CaV} 1.2)$ channel $\mathrm{C}$-terminus fragment is a bi-modal vasodilator. J Physiol 591:2987-2998.

Barbato JC (2005) Nicorandil: the drug that keeps on giving. Hypertension 46: 647-648.
Barlow CA, Rose P, Pulver-Kaste RA, and Lounsbury KM (2006) Excitationtranscription coupling in smooth muscle. $J$ Physiol 570:59-64.

Barra JG, Armentano RL, Levenson J, Fischer EI, Pichel RH, and Simon A (1993) Assessment of smooth muscle contribution to descending thoracic aortic elastic mechanics in conscious dogs. Circ Res 73:1040-1050.

Barst RJ, McGoon M, Torbicki A, Sitbon O, Krowka MJ, Olschewski H, and Gaine S (2004) Diagnosis and differential assessment of pulmonary arterial hypertension. $J$ Am Coll Cardiol 43(12, Suppl S)40S-47S.

Bayliss WM (1902) On the local reactions of the arterial wall to changes of internal pressure. J Physiol 28:220-231.

Bayoglu B, Arslan C, Gode S, Kaya Dagistanli F, Arapi B, Burc Deser S, Dirican A and Cengiz M (2014) The severity of internal carotid artery stenosis is associated with the cyclin-dependent kinase inhibitor $2 \mathrm{~A}$ gene expression. $J$ Atheroscler Thromb 21:659-671.

Beech DJ (2005) Emerging functions of 10 types of TRP cationic channel in vascular smooth muscle. Clin Exp Pharmacol Physiol 32:597-603.

Beech DJ (2012) Integration of transient receptor potential canonical channels with lipids. Acta Physiol (Oxf) 204:227-237.

Beech DJ (2013) Characteristics of transient receptor potential canonical calciumpermeable channels and their relevance to vascular physiology and disease. Circ $J$ 77:570-579.

Bell RD, Long X, Lin M, Bergmann JH, Nanda V, Cowan SL, Zhou Q, Han Y, Spector DL, and Zheng D, et al. (2014) Identification and initial functional characterization of a human vascular cell-enriched long noncoding RNA. Arterioscler Thromb Vasc Biol 34:1249-1259.

Ben-Shlomo Y, Spears M, Boustred C, May M, Anderson SG, Benjamin EJ, Boutouyrie P, Cameron J, Chen C-H, and Cruickshank JK, et al. (2014) Aortic pulse wave velocity improves cardiovascular event prediction: an individual participant meta-analysis of prospective observational data from 17,635 subjects. J Am Coll Cardiol 63:636-646.

Benoit JN, Barrowman JA, Harper SL, Kvietys PR, and Granger DN (1984) Role of humoral factors in the intestinal hyperemia associated with chronic portal hypertension. Am J Physiol 247:G486-G493.

Berridge MJ (2006) Calcium microdomains: organization and function. Cell Calcium 40:405-412.

Bertero T, Cottrill K, Krauszman A, Lu Y, Annis S, Hale A, Bhat B, Waxman AB, Chau BN, and Kuebler WM, et al. (2015) The microRNA-130/301 family controls vasoconstriction in pulmonary hypertension. J Biol Chem 290:2069-2085.

Bhattachariya A, Dahan D, Turczyńska KM, Swärd K, Hellstrand P, and Albinsson S (2014) Expression of microRNAs is essential for arterial myogenic tone and pressure-induced activation of the PI3-kinase/Akt pathway. Cardiovasc Res 101: 288-296.

Bierer R, Nitta CH, Friedman J, Codianni S, de Frutos S, Dominguez-Bautista JA, Howard TA, Resta TC, and Bosc LV (2011) NFATc3 is required for chronic hypoxiainduced pulmonary hypertension in adult and neonatal mice. Am J Physiol Lung Cell Mol Physiol 301:L872-L880.

Billaud M, Lohman AW, Johnstone SR, Biwer LA, Mutchler S, and Isakson BE (2014) Regulation of cellular communication by signaling microdomains in the blood vessel wall. Pharmacol Rev 66:513-569.

Billington N, Beach JR, Heissler SM, Remmert K, Guzik-Lendrum S, Nagy A, Takagi Y, Shao L, Li D, and Yang Y, et al. (2015) Myosin 18A coassembles with nonmuscle myosin 2 to form mixed bipolar filaments. Curr Biol 25:942-948.

Billington N, Wang A, Mao J, Adelstein RS, and Sellers JR (2013) Characterization of three full-length human nonmuscle myosin II paralogs. $J$ Biol Chem 288: 33398-33410.

Bird IM, Zhang L, and Magness RR (2003) Possible mechanisms underlying pregnancy-induced changes in uterine artery endothelial function. Am J Physiol Regul Integr Comp Physiol 284:R245-R258.

Birukov KG, Stepanova OV, Nanaev AK, and Shirinsky VP (1991) Expression of calponin in rabbit and human aortic smooth muscle cells. Cell Tissue Res 266: 579-584

Blacher J, Guerin AP, Pannier B, Marchais SJ, Safar ME, and London GM (1999) Impact of aortic stiffness on survival in end-stage renal disease. Circulation 99: $2434-2439$.

Boedtkjer DM, Matchkov VV, Boedtkjer E, Nilsson H, and Aalkjaer C (2008) Vasomotion has chloride-dependency in rat mesenteric small arteries. Pflugers Arch 457:389-404.

Boedtkjer E, Praetorius J, Matchkov VV, Stankevicius E, Mogensen S, Füchtbaue AC, Simonsen U, Füchtbauer EM, and Aalkjaer C (2011) Disruption of $\mathrm{Na}+, \mathrm{HCO}_{3}{ }^{-}$ cotransporter NBCn1 (slc4a7) inhibits NO-mediated vasorelaxation, smooth muscle $\mathrm{Ca}^{2+}$ sensitivity, and hypertension development in mice. Circulation 124: $1819-1829$

Boettger T, Beetz N, Kostin S, Schneider J, Krüger M, Hein L, and Braun T (2009) Acquisition of the contractile phenotype by murine arterial smooth muscle cells depends on the Mir143/145 gene cluster. J Clin Invest 119:2634-2647.

Bogaard HJ, Mizuno S, Hussaini AA, Toldo S, Abbate A, Kraskauskas D, Kasper M Natarajan R, and Voelkel NF (2011) Suppression of histone deacetylases worsens right ventricular dysfunction after pulmonary artery banding in rats. Am J Respir Crit Care Med 183:1402-1410.

Bomzon A and Blendis LM (1994) The nitric oxide hypothesis and the hyperdynamic circulation in cirrhosis. Hepatology 20:1343-1350.

Boon RA, Seeger T, Heydt S, Fischer A, Hergenreider E, Horrevoets AJ, Vinciguerra M, Rosenthal N, Sciacca S, and Pilato M, et al. (2011) MicroRNA-29 in aortic dilation: implications for aneurysm formation. Circ Res 109:1115-1119.

Bootman MD and Berridge MJ (1996) Subcellular Ca2+ signals underlying waves and graded responses in HeLa cells. Curr Biol 6:855-865.

Boucher JM, Peterson SM, Urs S, Zhang C, and Liaw L (2011) The miR-143/145 cluster is a novel transcriptional target of Jagged-1/Notch signaling in vascular smooth muscle cells. J Biol Chem 286:28312-28321.

Bracken CP, Li X, Wright JA, Lawrence DM, Pillman KA, Salmanidis M, Anderson MA, Dredge BK, Gregory PA, and Tsykin A, et al. (2014) Genome-wide 
identification of miR-200 targets reveals a regulatory network controlling cell invasion. EMBO $J$ 33:2040-2056.

Bradley AB and Morgan KG (1985) Cellular Ca2+ monitored by aequorin in adenosine-mediated smooth muscle relaxation. Am J Physiol 248:H109-H117.

Brenner R, Peréz GJ, Bonev AD, Eckman DM, Kosek JC, Wiler SW, Patterson AJ, Nelson MT, and Aldrich RW (2000) Vasoregulation by the beta1 subunit of the calcium-activated potassium channel. Nature 407:870-876

Brock M, Haider TJ, Vogel J, Gassmann M, Speich R, Trenkmann M, Ulrich S, Kohler M, and Huber LC (2015) The hypoxia-induced microRNA-130a controls pulmonary smooth muscle cell proliferation by directly targeting CDKN1A. Int $J$ Biochem Cell Biol 61:129-137.

Brooke BS, Karnik SK, and Li DY (2003) Extracellular matrix in vascular morphogenesis and disease: structure versus signal. Trends Cell Biol 13:51-56.

Brown DI and Griendling KK (2015) Regulation of signal transduction by reactive oxygen species in the cardiovascular system. Circ Res 116:531-549.

Brunner M, Cooper-DeHoff RM, Gong Y, Karnes JH, Langaee TY, Pepine CJ, Johnson JA, and Investigators I; INVEST Investigators (2007) Factors influencing blood pressure response to trandolapril add-on therapy in patients taking verapamil SR (from the International Verapamil SR/Trandolapril [INVEST] Study). Am $J$ Cardiol 99:1549-1554.

Bryan J (1990) Caldesmon: fragments, sequence, and domain mapping. Ann NY Acad Sci 599:100-110.

Bulley S and Jaggar JH (2014) $\mathrm{Cl}^{-}$channels in smooth muscle cells. Pflugers Arch 466: $861-872$

Bulley S, Neeb ZP, Burris SK, Bannister JP, Thomas-Gatewood CM, Jangsangthong W, and Jaggar JH (2012) TMEM16A/ANO1 channels contribute to the myogenic response in cerebral arteries. Circ Res 111:1027-1036.

Cabanillas ME, Hu MI, Durand JB, and Busaidy NL (2011) Challenges associated with tyrosine kinase inhibitor therapy for metastatic thyroid cancer. $J$ Thyroid Res 2011:985780

Cannon RO 3rd (1998) Role of nitric oxide in cardiovascular disease: focus on the endothelium. Clin Chem 44:1809-1819.

Cardinale JP, Sriramula S, Pariaut R, Guggilam A, Mariappan N, Elks CM, and Francis J (2010) HDAC inhibition attenuates inflammatory, hypertrophic, and hypertensive responses in spontaneously hypertensive rats. Hypertension 56: 437-444.

Carrión AM, Link WA, Ledo F, Mellström B, and Naranjo JR (1999) DREAM is a Ca2 +-regulated transcriptional repressor. Nature 398:80-84.

Carson P, Ziesche S, Johnson G, and Cohn JN; Vasodilator-Heart Failure Trial Study Group (1999) Racial differences in response to therapy for heart failure: analysis of the vasodilator-heart failure trials. J Card Fail 5:178-187.

Carter RW and Kanagy NL (2003) Mechanism of enhanced calcium sensitivity and alpha 2 -AR vasoreactivity in chronic NOS inhibition hypertension. Am J Physiol Heart Circ Physiol 284:H309-H316.

Cartin L, Lounsbury KM, and Nelson MT (2000) Coupling of $\mathrm{Ca}(2+)$ to CREB activation and gene expression in intact cerebral arteries from mouse : roles of ryanodine receptors and voltage-dependent $\mathrm{Ca}(2+)$ channels. Circ Res 86:760-767.

Castro R, Rivera I, Struys EA, Jansen EE, Ravasco P, Camilo ME, Blom HJ, Jakobs C, and Tavares de Almeida I (2003) Increased homocysteine and Sadenosylhomocysteine concentrations and DNA hypomethylation in vascular disease. Clin Chem 49:1292-1296.

Chacko S, Conti MA, and Adelstein RS (1977) Effect of phosphorylation of smooth muscle myosin on actin activation and $\mathrm{Ca} 2+$ regulation. Proc Natl Acad Sci USA 74:129-133.

Chan MC, Hilyard AC, Wu C, Davis BN, Hill NS, Lal A, Lieberman J, Lagna G, and Hata A (2010) Molecular basis for antagonism between PDGF and the TGFbeta family of signalling pathways by control of miR-24 expression. EMBO J 29:559-573.

Chang MY, Huang DY, Ho FM, Huang KC, and Lin WW (2012) PKC-dependent human monocyte adhesion requires AMPK and Syk activation. PLoS One 7:e40999.

Chen A, Karolczak-Bayatti M, Sweeney M, Treumann A, Morrissey K, Ulrich SM, Europe-Finner GN, and Taggart MJ (2013) Lysine deacetylase inhibition promotes relaxation of arterial tone and C-terminal acetylation of HSPB6 (Hsp20) in vascular smooth muscle cells. Physiol Rep 1:e00127.

Chen CP, Chen X, Qiao YN, Wang P, He WQ, Zhang CH, Zhao W, Gao YQ, Chen C, and Tao T, et al. (2015) In vivo roles for myosin phosphatase targeting subunit-1 phosphorylation sites T694 and T852 in bladder smooth muscle contraction. $J$ Physiol 593:681-700.

Chen FC and Brozovich FV (2008) Gene expression profiles of vascular smooth muscle show differential expression of mitogen-activated protein kinase pathways during captopril therapy of heart failure. J Vasc Res 45:445-454.

Chen FC, Ogut O, Rhee AY, Hoit BD, and Brozovich FV (2006) Captopril prevents myosin light chain phosphatase isoform switching to preserve normal cGMPmediated vasodilatation. $J \mathrm{Mol}$ Cell Cardiol 41:488-495.

Cheng EP, Yuan C, Navedo MF, Dixon RE, Nieves-Cintrón M, Scott JD, and Santana $\mathrm{LF}$ (2011) Restoration of normal L-type $\mathrm{Ca} 2+$ channel function during Timothy syndrome by ablation of an anchoring protein. Circ Res 109:255-261.

Cheng J, Ke Q, Jin Z, Wang H, Kocher O, Morgan JP, Zhang J, and Crumpacker CS (2009) Cytomegalovirus infection causes an increase of arterial blood pressure. PLoS Pathog 5:e1000427.

Chettimada S, Ata H, Rawat DK, Gulati S, Kahn AG, Edwards JG, and Gupte SA (2014) Contractile protein expression is upregulated by reactive oxygen species in aorta of Goto-Kakizaki rat. Am J Physiol Heart Circ Physiol 306:H214-H224.

Cheung P, Allis CD, and Sassone-Corsi P (2000) Signaling to chromatin through histone modifications. Cell 103:263-271.

Chi M, Zhou Y, Vedamoorthyrao S, Babu GJ, and Periasamy M (2008) Ablation of smooth muscle myosin heavy chain SM2 increases smooth muscle contraction and results in postnatal death in mice. Proc Natl Acad Sci USA 105:18614-18618.

Childs TJ, Watson MH, Sanghera JS, Campbell DL, Pelech SL, and Mak AS (1992) Phosphorylation of smooth muscle caldesmon by mitogen-activated protein (MAP) kinase and expression of MAP kinase in differentiated smooth muscle cells. $J$ Biol Chem 267:22853-22859.

Choe N, Kwon JS, Kim JR, Eom GH, Kim Y, Nam KI, Ahn Y, Kee HJ, and Kook H (2013) The microRNA miR-132 targets Lrrfip1 to block vascular smooth muscle cell proliferation and neointimal hyperplasia. Atherosclerosis 229:348-355.

Choudhary C, Kumar C, Gnad F, Nielsen ML, Rehman M, Walther TC, Olsen JV, and Mann M (2009) Lysine acetylation targets protein complexes and co-regulates major cellular functions. Science 325:834-840

Chu TF, Rupnick MA, Kerkela R, Dallabrida SM, Zurakowski D, Nguyen L, Woulfe K, Pravda E, Cassiola F, and Desai J, et al. (2007) Cardiotoxicity associated with tyrosine kinase inhibitor sunitinib. Lancet 370:2011-2019.

Chutkow WA, Pu J, Wheeler MT, Wada T, Makielski JC, Burant CF, and McNally EM (2002) Episodic coronary artery vasospasm and hypertension develop in the absence of Sur2 K(ATP) channels. J Clin Invest 110:203-208.

Cipolla MJ, Gokina NI, and Osol G (2002) Pressure-induced actin polymerization in vascular smooth muscle as a mechanism underlying myogenic behavior. FASEB $J$ 16:72-76

Clark JM and Glagov S (1985) Transmural organization of the arterial media. The lamellar unit revisited. Arteriosclerosis 5:19-34.

Coffman TM and Crowley SD (2008) Kidney in hypertension: guyton redux. Hypertension 51:811-816.

Collier ML, Ji G, Wang Y, and Kotlikoff MI (2000) Calcium-induced calcium release in smooth muscle: loose coupling between the action potential and calcium release. $J$ Gen Physiol 115:653-662.

Congrains A, Kamide K, Katsuya T, Yasuda O, Oguro R, Yamamoto K, Ohishi M, and Rakugi H (2012a) CVD-associated non-coding RNA, ANRIL, modulates expression of atherogenic pathways in VSMC. Biochem Biophys Res Commun 419:612-616.

Congrains A Kamide K, Oguro R, Yasuda O, Miyata K, Yamamoto E, Kawai T, Kusunoki H, Yamamoto H, and Takeya Y, et al. (2012b) Genetic variants at the 9p21 locus contribute to atherosclerosis through modulation of ANRIL and CDKN2A/B. Atherosclerosis 220:449-455.

Connell LE and Helfman DM (2006) Myosin light chain kinase plays a role in the regulation of epithelial cell survival. J Cell Sci 119:2269-2281.

Connolly MJ and Aaronson PI (2011) Key role of the RhoA/Rho kinase system in pulmonary hypertension. Pulm Pharmacol Ther 24:1-14.

Cooke JP and Marshall JM (2005) Mechanisms of Raynaud's disease. Vasc Med 10 293-307.

Corbi G, Conti V, Russomanno G, Longobardi G, Furgi G, Filippelli A, and Ferrara N (2013) Adrenergic signaling and oxidative stress: a role for sirtuins? Front Physiol 4:324.

Cox RH and Rusch NJ (2002) New expression profiles of voltage-gated ion channels in arteries exposed to high blood pressure. Microcirculation 9:243-257.

Cremo CR, Wang F, Facemyer K, and Sellers JR (2001) Phosphorylation-dependent regulation is absent in a nonmuscle heavy meromyosin construct with one complete head and one head lacking the motor domain. $J$ Biol Chem 276:41465-41472.

Crowley JJ, Sullivan PF, and McLeod HL (2009) Pharmacogenomic genome-wide association studies: lessons learned thus far. Pharmacogenomics 10:161-163.

Crowley SD, Gurley SB, Oliverio MI, Pazmino AK, Griffiths R, Flannery PJ, Spurney RF, Kim HS, Smithies O, and Le TH, et al. (2005) Distinct roles for the kidney and systemic tissues in blood pressure regulation by the renin-angiotensin system. $J$ Clin Invest 115:1092-1099.

Cruickshank K, Riste L, Anderson SG, Wright JS, Dunn G, and Gosling RG (2002 Aortic pulse-wave velocity and its relationship to mortality in diabetes and glucose intolerance: an integrated index of vascular function? Circulation 106:2085-2090.

Cushman WC, Reda DJ, Perry HM, Williams D, Abdellatif M, and Materson BJ; Department of Veterans Affairs Cooperative Study Group on Antihypertensive Agents (2000) Regional and racial differences in response to antihypertensive medication use in a randomized controlled trial of men with hypertension in the United States. Arch Intern Med 160:825-831.

Dahan D, Ekman M, Larsson-Callerfelt AK, Turczyńska K, Boettger T, Braun T, Swärd K, and Albinsson S (2014) Induction of angiotensin-converting enzyme after miR-143/145 deletion is critical for impaired smooth muscle contractility. Am J Physiol Cell Physiol 307:C1093-C1101.

Dam VS, Boedtkjer DM, Nyvad J, Aalkjaer C, and Matchkov V (2014) TMEM16A knockdown abrogates two different $\mathrm{Ca}(2+)$-activated $\mathrm{Cl}(-)$ currents and contractility of smooth muscle in rat mesenteric small arteries. Pflugers Arch 466: 1391-1409.

D'Angelo G, Graceffa P, Wang CA, Wrangle J, and Adam LP (1999) Mammal-specific, ERK-dependent, caldesmon phosphorylation in smooth muscle. Quantitation using novel anti-phosphopeptide antibodies. J Biol Chem 274:30115-30121.

Das S and Halushka MK (2015) Extracellular vesicle microRNA transfer in cardiovascular disease. Cardiovasc Pathol 24:199-206.

Davis AJ, Shi J, Pritchard HA, Chadha PS, Leblanc N, Vasilikostas G, Yao Z Verkman AS, Albert AP, and Greenwood IA (2013) Potent vasorelaxant activity of the TMEM16A inhibitor T16A(inh) -A01. Br J Pharmacol 168:773-784.

Davis MJ and Hill MA (1999) Signaling mechanisms underlying the vascular myogenic response. Physiol Rev 79:387-423.

Davis-Dusenbery BN, Chan MC, Reno KE, Weisman AS, Layne MD, Lagna G, and Hata A (2011) down-regulation of Kruppel-like factor-4 (KLF4) by microRNA$143 / 145$ is critical for modulation of vascular smooth muscle cell phenotype by transforming growth factor-beta and bone morphogenetic protein 4. J Biol Chem 286:28097-28110.

De La Cruz EM and Ostap EM (2004) Relating biochemistry and function in the myosin superfamily. Curr Opin Cell Biol 16:61-67.

Deng JT, Sutherland C, Brautigan DL, Eto M, and Walsh MP (2002) Phosphorylation of the myosin phosphatase inhibitors, CPI-17 and PHI-1, by integrin-linked kinase. Biochem J 367:517-524.

Dessy C, Kim I, Sougnez CL, Laporte R, and Morgan KG (1998) A role for MAP kinase in differentiated smooth muscle contraction evoked by alpha-adrenoceptor stimulation. Am J Physiol 275:C1081-C1086. 
Devine CE and Somlyo AP (1971) Thick filaments in vascular smooth muscle. J Cell Biol 49:636-649.

Dhanasekaran DN, Kashef K, Lee CM, Xu H, and Reddy EP (2007) Scaffold proteins of MAP-kinase modules. Oncogene 26:3185-3202.

Dietrich A, Mederos y Schnitzler M, Emmel J, Kalwa H, Hofmann T, and Gudermann $\mathrm{T}$ (2003) N-linked protein glycosylation is a major determinant for basal TRPC3 and TRPC6 channel activity. $J$ Biol Chem 278:47842-47852.

Dillon PF, Aksoy MO, Driska SP, and Murphy RA (1981) Myosin phosphorylation and the cross-bridge cycle in arterial smooth muscle. Science 211:495-497.

Dillon PF and Murphy RA (1982) Tonic force maintenance with reduced shortening velocity in arterial smooth muscle. Am J Physiol 242:C102-C108.

Ding SY, Lee MJ, Summer R, Liu L, Fried SK, and Pilch PF (2014) Pleiotropic effects of cavin-1 deficiency on lipid metabolism. J Biol Chem 289:8473-8483.

Dioum EM, Chen R, Alexander MS, Zhang Q, Hogg RT, Gerard RD, and Garcia JA (2009) Regulation of hypoxia-inducible factor 2alpha signaling by the stressresponsive deacetylase sirtuin 1 . Science 324:1289-1293.

Dirksen WP, Vladic F, and Fisher SA (2000) A myosin phosphatase targeting subunit isoform transition defines a smooth muscle developmental phenotypic switch. Am J Physiol Cell Physiol 278:C589-C600.

Dobrin PB (1978) Mechanical properties of arterises. Physiol Rev 58:397-460

Dong C, Yoon W, and Goldschmidt-Clermont PJ (2002) DNA methylation and atherosclerosis. J Nutr 132(8, Suppl)2406S-2409S

Dou D, Ma H, Zheng X, Ying L, Guo Y, Yu X, and Gao Y (2010) Degradation of leucine zipper-positive isoform of MYPT1 may contribute to development of nitrate tolerance. Cardiovasc Res 86:151-159.

Drew JS, Moos C, and Murphy RA (1991) Localization of isoactins in isolated smooth muscle thin filaments by double gold immunolabeling. Am $J$ Physiol 260: C1332-C1340.

Duband JL, Gimona M, Scatena M, Sartore S, and Small JV (1993) Calponin and SM 22 as differentiation markers of smooth muscle: spatiotemporal distribution during avian embryonic development. Differentiation 55:1-11.

Dustan HP, Roccella EJ, and Garrison HH (1996) Controlling hypertension. A research success story. Arch Intern Med 156:1926-1935.

Dzau VJ (2001) Theodore Cooper Lecture: Tissue angiotensin and pathobiology of vascular disease: a unifying hypothesis. Hypertension 37:1047-1052.

Earley S and Brayden JE (2015) Transient receptor potential channels in the vasculature. Physiol Rev 95:645-690.

Earley S, Pauyo T, Drapp R, Tavares MJ, Liedtke W, and Brayden JE (2009) TRPV4dependent dilation of peripheral resistance arteries influences arterial pressure Am J Physiol Heart Circ Physiol 297:H1096-H1102.

Eddinger TJ, Meer DP, Miner AS, Meehl J, Rovner AS, and Ratz PH (2007) Potent inhibition of arterial smooth muscle tonic contractions by the selective myosin II inhibitor, blebbistatin. J Pharmacol Exp Ther 320:865-870.

Ehret GB (2010) Genome-wide association studies: contribution of genomics to understanding blood pressure and essential hypertension. Curr Hypertens Rep 12:17-25.

EL-Mezgueldi M and Marston SB (1996) The effects of smooth muscle calponin on the strong and weak myosin binding sites of F-actin. J Biol Chem 271:28161-28167.

El-Touhky A, Given AM, Cochard A, and Brozovich FV (2005) PHI-1 induced enhancement of myosin phosphorylation in chicken smooth muscle. FEBS Lett $\mathbf{5 7 9}$ $4271-4277$.

El-Toukhy A, Given AM, Ogut O, and Brozovich FV (2006) PHI-1 interacts with the catalytic subunit of myosin light chain phosphatase to produce a $\mathrm{Ca}(2+)$ independent increase in $\mathrm{MLC}(20)$ phosphorylation and force in avian smooth muscle. FEBS Lett 580:5779-5784.

El-Yazbi AF, Abd-Elrahman KS, and Moreno-Dominguez A (2015) PKC-mediated cerebral vasoconstriction: Role of myosin light chain phosphorylation versus actin cytoskeleton reorganization. Biochem Pharmacol 95:263-278.

El-Yazbi AF, Johnson RP, Walsh EJ, Takeya K, Walsh MP, and Cole WC (2010) Pressure-dependent contribution of Rho kinase-mediated calcium sensitization in serotonin-evoked vasoconstriction of rat cerebral arteries. $J$ Physiol $\mathbf{5 8 8}$ $1747-1762$.

Ellison PA, Sellers JR, and Cremo CR (2000) Kinetics of smooth muscle heavy meromyosin with one thiophosphorylated head. J Biol Chem 275:15142-15151.

Essin K, Welling A, Hofmann F, Luft FC, Gollasch M, and Moosmang S (2007) In direct coupling between Cav1.2 channels and ryanodine receptors to generate $\mathrm{Ca} 2+$ sparks in murine arterial smooth muscle cells. J Physiol 584:205-219.

Esteban V, Méndez-Barbero N, Jiménez-Borreguero LJ, Roqué M, Novensá L, García-Redondo AB, Salaices M, Vila L, Arbonés ML, and Campanero MR, et al. (2011) Regulator of calcineurin 1 mediates pathological vascular wall remodeling. J Exp Med 208:2125-2139.

Eto M, Karginov A, and Brautigan DL (1999) A novel phosphoprotein inhibitor of protein type-1 phosphatase holoenzymes. Biochemistry 38:16952-16957.

Eto M, Kitazawa T, Matsuzawa F, Aikawa S, Kirkbride JA, Isozumi N, Nishimura Y Brautigan DL, and Ohki SY (2007) Phosphorylation-induced conformational switching of CPI-17 produces a potent myosin phosphatase inhibitor. Structure $\mathbf{1 5}$ 1591-1602.

Eto M, Ohmori T, Suzuki M, Furuya K, and Morita F (1995) A novel protein phosphatase-1 inhibitory protein potentiated by protein kinase C. Isolation from porcine aorta media and characterization. J Biochem 118:1104-1107.

Europe-Finner GN, Karolczak-Bayatti M, and Taggart MJ (2015) The multifaceted KDAC8: a smooth muscle contractile regulator. Trends Pharmacol Sci 36:493.

Farkas L, Gauldie J, Voelkel NF, and Kolb M (2011) Pulmonary hypertension and idiopathic pulmonary fibrosis: a tale of angiogenesis, apoptosis, and growth factors. Am J Respir Cell Mol Biol 45:1-15.

Fatigati V and Murphy RA (1984) Actin and tropomyosin variants in smooth muscles. Dependence on tissue type. J Biol Chem 259:14383-14388.

Fediuk J and Dakshinamurti S (2015) A role for actin polymerization in persistent pulmonary hypertension of the newborn. Can J Physiol Pharmacol 93:185-194.

Fernández-Fernández JM, Tomás M, Vázquez E, Orio P, Latorre R, Sentí M, Marrugat J, and Valverde MA (2004) Gain-of-function mutation in the KCNMB1 potassium channel subunit is associated with low prevalence of diastolic hypertension. $J$ Clin Invest 113:1032-1039.

Findeisen HM, Gizard F, Zhao Y, Qing H, Heywood EB, Jones KL, Cohn D, and Bruemmer D (2011) Epigenetic regulation of vascular smooth muscle cell proliferation and neointima formation by histone deacetylase inhibition. Arterioscler Thromb Vasc Biol 31:851-860.

Fisher SA (2010) Vascular smooth muscle phenotypic diversity and function. Physiol Genomics 42A:169-187.

Fleenor BS, Marshall KD, Durrant JR, Lesniewski LA, and Seals DR (2010) Arterial stiffening with ageing is associated with transforming growth factor-B1-related changes in adventitial collagen: reversal by aerobic exercise. $J$ Physiol 588: 3971-3982.

Fleenor BS, Sindler AL, Eng JS, Nair DP, Dodson RB, and Seals DR (2012) Sodium nitrite de-stiffening of large elastic arteries with aging: role of normalization of advanced glycation end-products. Exp Gerontol 47:588-594.

Force T, Krause DS, and Van Etten RA (2007) Molecular mechanisms of cardiotoxicity of tyrosine kinase inhibition. Nat Rev Cancer 7:332-344

Foskett JK, White C, Cheung KH, and Mak DO (2007) Inositol trisphosphate receptor Ca2+ release channels. Physiol Rev 87:593-658.

Foster DB, Shen LH, Kelly J, Thibault P, Van Eyk JE, and Mak AS (2000) Phosphorylation of caldesmon by p21-activated kinase. Implications for the $\mathrm{Ca}(2+)$ sensitivity of smooth muscle contraction. J Biol Chem 275:1959-1965.

Frid MG, Shekhonin BV, Koteliansky VE, and Glukhova MA (1992) Phenotypic changes of human smooth muscle cells during development: late expression of heavy caldesmon and calponin. Dev Biol 153:185-193

Fry JL, Shiraishi Y, Turcotte R, Yu X, Gao YZ, Akiki R, Bachschmid M, Zhang Y, Morgan KG, and Cohen RA, et al. (2015) Vascular Smooth Muscle Sirtuin-1 Protects Against Aortic Dissection During Angiotensin II-Induced Hypertension. J Am Heart Assoc 4:e02384

Fukao M, Mason HS, Britton FC, Kenyon JL, Horowitz B, and Keef KD (1999) Cyclic GMP-dependent protein kinase activates cloned BKCa channels expressed in mammalian cells by direct phosphorylation at serine 1072. J Biol Chem 274: 10927-10935.

Furchgott RF (1999) Endothelium-derived relaxing factor: discovery, early studies, and identification as nitric oxide. Biosci Rep 19:235-251.

Furchgott RF and Zawadzki JV (1980) The obligatory role of endothelial cells in the relaxation of arterial smooth muscle by acetylcholine. Nature 288:373-376.

Fürst DO, Cross RA, De Mey J, and Small JV (1986) Caldesmon is an elongated, flexible molecule localized in the actomyosin domains of smooth muscle. EMBO $J$ 5:251-257.

Gabbiani G, Schmid E, Winter S, Chaponnier C, de Ckhastonay C, Vandekerckhove J, Weber K, and Franke WW (1981) Vascular smooth muscle cells differ from other smooth muscle cells: predominance of vimentin filaments and a specific alpha-type actin. Proc Natl Acad Sci USA 78:298-302.

Gagnon A, Wilson RD, Audibert F, Allen VM, Blight C, Brock JA, Désilets VA, Johnson JA, Langlois S, and Summers A, et al.; Society of Obstetricians and Gynaecologists of Canada Genetics Committee (2008) Obstetrical complications associated with abnormal maternal serum markers analytes. J Obstet Gynaecol Can 30:918-949.

Gallagher PJ, Herring BP, Griffin SA, and Stull JT (1991) Molecular characterization of a mammalian smooth muscle myosin light chain kinase. J Biol Chem 266: $23936-23944$.

Gallant C, Appel S, Graceffa P, Leavis P, Lin JJ, Gunning PW, Schevzov G, Chaponnier C, DeGnore J, and Lehman W, et al. (2011) Tropomyosin variants describe distinct functional subcellular domains in differentiated vascular smooth muscle cells. Am J Physiol Cell Physiol 300:C1356-C1365.

Gamble G, Zorn J, Sanders G, MacMahon S, and Sharpe N (1994) Estimation of arterial stiffness, compliance, and distensibility from M-mode ultrasound measurements of the common carotid artery. Stroke 25:11-16.

Gangopadhyay SS, Barber AL, Gallant C, Grabarek Z, Smith JL, and Morgan KG (2003) Differential functional properties of calmodulin-dependent protein kinase IIgamma variants isolated from smooth muscle. Biochem $J$ 372:347-357.

Gangopadhyay SS, Gallant C, Sundberg EJ, Lane WS, and Morgan KG (2008) Regulation of $\mathrm{Ca} 2+/$ calmodulin kinase II by a small C-terminal domain phosphatase. Biochem J 412:507-516.

Gangopadhyay SS, Kengni E, Appel S, Gallant C, Kim HR, Leavis P, DeGnore J, and Morgan KG (2009) Smooth muscle archvillin is an ERK scaffolding protein. J Biol Chem 284:17607-17615.

Gangopadhyay SS, Takizawa N, Gallant C, Barber AL, Je HD, Smith TC, Luna EJ, and Morgan KG (2004) Smooth muscle archvillin: a novel regulator of signaling and contractility in vascular smooth muscle. J Cell Sci 117:5043-5057.

Gao P, Xu TT, Lu J, Li L, Xu J, Hao DL, Chen HZ, and Liu DP (2014a) Overexpression of SIRT1 in vascular smooth muscle cells attenuates angiotensin II induced vascular remodeling and hypertension in mice. J $\mathrm{Mol} \mathrm{Med} \mathrm{(Berl)} \mathrm{92:}$ 347-357.

Gao YZ, Saphirstein RJ, Yamin RY, Suki B, and Morgan KG (2014b) Aging impairs smooth muscle mediated regulation of aortic stiffness: a defect in shock absorption function? Am J Physiol Heart Circ Physiol 307:H1252-H1261.

Gaylinn BD, Eddinger TJ, Martino PA, Monical PL, Hunt DF, and Murphy RA (1989) Expression of nonmuscle myosin heavy and light chains in smooth muscle. Am $J$ Physiol 257:C997-C1004.

Geraci MW, Gao B, Hoshikawa Y, Yeager ME, Tuder RM, and Voelkel NF (2001a) Genomic approaches to research in pulmonary hypertension. Respir Res 2: $210-215$.

Geraci MW, Moore M, Gesell T, Yeager ME, Alger L, Golpon H, Gao B, Loyd JE, Tuder RM, and Voelkel NF (2001b) Gene expression patterns in the lungs of patients with primary pulmonary hypertension: a gene microarray analysis. Circ Res 88:555-562.

Ghofrani HA, D'Armini AM, Grimminger F, Hoeper MM, Jansa P, Kim NH, Mayer E, Simonneau G, Wilkins MR, and Fritsch A, et al.; CHEST-1 Study Group (2013a) 
Riociguat for the treatment of chronic thromboembolic pulmonary hypertension. $N$ Engl J Med 369:319-329.

Ghofrani HA, Galiè N, Grimminger F, Grünig E, Humbert M, Jing ZC, Keogh AM, Langleben D, Kilama MO, and Fritsch A, et al.; PATENT-1 Study Group (2013b) Riociguat for the treatment of pulmonary arterial hypertension. N Engl J Med 369: 330-340.

Giachini FR, Carneiro FS, Lima VV, Carneiro ZN, Dorrance AM, Tostes RC, and Webb RC (2009a) Sex differences in vascular expression and activation of STIM-1/Orai-1 during hypertension: focus on calcium regulation. FASEB $J \mathbf{2 3}$ : Suppl 1, 781-15.

Giachini FR, Chiao CW, Carneiro FS, Lima VV, Carneiro ZN, Dorrance AM, Tostes $\mathrm{RC}$, and Webb RC (2009b) Increased activation of stromal interaction molecule-1/ Orai-1 in aorta from hypertensive rats: a novel insight into vascular dysfunction. Hypertension 53:409-416.

Ginnan R, Sun LY, Schwarz JJ, and Singer HA (2012) MEF2 is regulated by CaMKII 2 and a HDAC4-HDAC5 heterodimer in vascular smooth muscle cells. Biochem J 444:105-114.

Ginnan R, Zou X, Pfleiderer PJ, Mercure MZ, Barroso M, and Singer HA (2013) Vascular smooth muscle cell motility is mediated by a physical and functional interaction of $\mathrm{Ca} 2+/$ calmodulin-dependent protein kinase III2 2 and Fyn. J Biol Chem 288:29703-29712.

Given AM, Ogut O, and Brozovich FV (2007) MYPT1 mutants demonstrate the importance of aa 888-928 for the interaction with PKGIalpha. Am J Physiol Cell Physiol 292:C432-C439.

Glagov S, Zarins CK, Masawa N, Xu CP, Bassiouny H, and Giddens DP (1993) Mechanical functional role of non-atherosclerotic intimal thickening. Front Med Biol Eng 5:37-43.

Golomb E, Ma X, Jana SS, Preston YA, Kawamoto S, Shoham NG, Goldin E, Conti MA, Sellers JR, and Adelstein RS (2004) Identification and characterization of nonmuscle myosin II-C, a new member of the myosin II family. J Biol Chem $\mathbf{2 7 9}$ : 2800-2808.

Gomez MF, Bosc LV, Stevenson AS, Wilkerson MK, Hill-Eubanks DC, and Nelson MT (2003) Constitutively elevated nuclear export activity opposes Ca2+-dependent NFATc3 nuclear accumulation in vascular smooth muscle: role of JNK2 and Crm-1. $J$ Biol Chem 278:46847-46853.

Gomez MF, Stevenson AS, Bonev AD, Hill-Eubanks DC, and Nelson MT (2002) Opposing actions of inositol 1,4,5-trisphosphate and ryanodine receptors on nuclear factor of activated T-cells regulation in smooth muscle. J Biol Chem 277: 37756-37764.

Goncharova EA, Shirinsky VP, Shevelev AY, Marston SB, and Vorotnikov AV (2001) Actomyosin cross-linking by caldesmon in non-muscle cells. FEBS Lett 497:113-117.

Gong MC, Cohen P, Kitazawa T, Ikebe M, Masuo M, Somlyo AP, and Somlyo AV (1992) Myosin light chain phosphatase activities and the effects of phosphatase inhibitors in tonic and phasic smooth muscle. J Biol Chem 267:14662-14668.

Gong Y, McDonough CW, Wang Z, Hou W, Cooper-DeHoff RM, Langaee TY, Beitelshees AL, Chapman AB, Gums JG, and Bailey KR, et al. (2012) Hypertension susceptibility loci and blood pressure response to antihypertensives results from the pharmacogenomic evaluation of antihypertensive responses study. Circ Cardiovasc Genet 5:686-691.

Gonzales AL, Yang Y, Sullivan MN, Sanders L, Dabertrand F, Hill-Eubanks DC, Nelson MT, and Earley S (2014) A PLC $\gamma 1$-dependent, force-sensitive signaling network in the myogenic constriction of cerebral arteries. Sci Signal 7:ra49.

Gonzalez Bosc LV, Wilkerson MK, Bradley KN, Eckman DM, Hill-Eubanks DC, and Nelson MT (2004) Intraluminal pressure is a stimulus for NFATc3 nuclear accumulation: role of calcium, endothelium-derived nitric oxide, and cGMPdependent protein kinase. J Biol Chem 279:10702-10709.

Gordienko DV and Bolton TB (2002) Crosstalk between ryanodine receptors and IP (3) receptors as a factor shaping spontaneous $\mathrm{Ca}(2+)$-release events in rabbit portal vein myocytes. J Physiol 542:743-762.

Gorenne I, Su X, and Moreland RS (2004) Caldesmon phosphorylation is catalyzed by two kinases in permeabilized and intact vascular smooth muscle. $J$ Cell Physiol 198:461-469.

Gosling RG and Budge MM (2003) Terminology for describing the elastic behavior of arteries. Hypertension 41:1180-1182.

Granger CB, McMurray JJ, Yusuf S, Held P, Michelson EL, Olofsson B, Ostergren J, Pfeffer MA, and Swedberg K; CHARM Investigators and Committees (2003) Effects of candesartan in patients with chronic heart failure and reduced left-ventricular systolic function intolerant to angiotensin-converting-enzyme inhibitors: the CHARM-Alternative trial. Lancet 362:772-776.

Greenwald SE (2007) Ageing of the conduit arteries. J Pathol 211:157-172.

Greenwood IA and Leblanc N (2007) Overlapping pharmacology of Ca2+-activated Cl- and K+ channels. Trends Pharmacol Sci 28:1-5.

Griendling KK, Lassègue B, Murphy TJ, and Alexander RW (1994) Angiotensin II receptor pharmacology. Adv Pharmacol 28:269-306.

Group IS; IONA Study Group (2002) Effect of nicorandil on coronary events in patients with stable angina: the Impact Of Nicorandil in Angina (IONA) randomised trial. Lancet 359:1269-1275.

Guarente L (2011) Franklin H. Epstein Lecture: Sirtuins, aging, and medicine. $N$ Engl J Med 364:2235-2244.

Guerin AP, Blacher J, Pannier B, Marchais SJ, Safar ME, and London GM (2001) Impact of aortic stiffness attenuation on survival of patients in end-stage renal failure. Circulation 103:987-992.

Gunst SJ and Zhang W (2008) Actin cytoskeletal dynamics in smooth muscle: a new paradigm for the regulation of smooth muscle contraction. Am J Physiol Cell Physiol 295:C576-C587.

Guo H, Huang R, Semba S, Kordowska J, Huh YH, Khalina-Stackpole Y, Mabuchi K, Kitazawa T, and Wang CL (2013) Ablation of smooth muscle caldesmon affects the relaxation kinetics of arterial muscle. Pflugers Arch 465:283-294.

Guo L, Qiu Z, Wei L, Yu X, Gao X, Jiang S, Tian H, Jiang C, and Zhu D (2012) The microRNA-328 regulates hypoxic pulmonary hypertension by targeting at insulin growth factor 1 receptor and L-type calcium channel- $\alpha 1 \mathrm{C}$. Hypertension 59: 1006-1013.

Guo RW, Wang H, Gao P, Li MQ, Zeng CY, Yu Y, Chen JF, Song MB, Shi YK, and Huang L (2009) An essential role for stromal interaction molecule 1 in neointima formation following arterial injury. Cardiovasc Res 81:660-668.

Gupta AK (2010) Racial differences in response to antihypertensive therapy: does one size fits all? Int $J$ Prev Med 1:217-219.

Gupta S and Li L (2015) Modulation of miRNAs in Pulmonary Hypertension. Int $J$ Hypertens 2015:169069.

Guvenc B, Ustunel C, Ozturk N, and Brozovich FV (2010) A dynamic approach reveals non-muscle myosin influences the overall smooth muscle cross-bridge cycling rate. FEBS Lett 584:2862-2866.

Guyton AC (1991) Blood pressure control-special role of the kidneys and body fluids. Science 252:1813-1816.

Haddock RE and Hill CE (2005) Rhythmicity in arterial smooth muscle. J Physiol 566:645-656.

Hai CM and Murphy RA (1989) Cross-bridge dephosphorylation and relaxation of vascular smooth muscle. Am J Physiol 256:C282-C287.

Haigis MC and Sinclair DA (2010) Mammalian sirtuins: biological insights and disease relevance. Annu Rev Pathol 5:253-295.

Hajjar I, Kotchen JM, and Kotchen TA (2006) Hypertension: trends in prevalence, incidence, and control. Annu Rev Public Health 27:465-490.

Hake S (2003) MicroRNAs: a role in plant development. Curr Biol 13:R851-R852.

Haldeman BD, Brizendine RK, Facemyer KC, Baker JE, and Cremo CR (2014) The kinetics underlying the velocity of smooth muscle myosin filament sliding on actin filaments in vitro. J Biol Chem 289:21055-21070.

Hamden SS, Schroeter MM, and Chalovich JM (2010) Phosphorylation of caldesmon at sites between residues 627 and 642 attenuates inhibitory activity and contributes to a reduction in Ca2+-calmodulin affinity. Biophys $J$ 99:1861-1868.

Hammond SM, Boettcher S, Caudy AA, Kobayashi R, and Hannon GJ (2001) Argonaute2, a link between genetic and biochemical analyses of RNAi. Science 293: 1146-1150.

Han YS and Brozovich FV (2013) Altered reactivity of tertiary mesenteric arteries following acute myocardial ischemia. J Vasc Res 50:100-108.

Hartshorne DJ, Ito M, and Erdödi F (1998) Myosin light chain phosphatase: subunit composition, interactions and regulation. J Muscle Res Cell Motil 19:325-341.

Hartshorne DJ, Ito M, and Erdödi F (2004) Role of protein phosphatase type 1 in contractile functions: myosin phosphatase. J Biol Chem 279:37211-37214.

Hasegawa Y and Morita F (1992) Role of 17-kDa essential light chain isoforms of aorta smooth muscle myosin. $J$ Biochem 111:804-809.

Havulinna AS, Kettunen J, Ukkola O, Osmond C, Eriksson JG, Kesäniemi YA, Jula A, Peltonen L, Kontula K, and Salomaa V, et al. (2013) A blood pressure genetic risk score is a significant predictor of incident cardiovascular events in 32,669 individuals. Hypertension 61:987-994.

He Q, Tan J, Yu B, Shi W, and Liang K (2015) Long noncoding RNA HIF1A-AS1A reduces apoptosis of vascular smooth muscle cells: implications for the pathogenesis of thoracoabdominal aorta aneurysm. Pharmazie 70:310-315.

Hedges JC, Oxhorn BC, Carty M, Adam LP, Yamboliev IA, and Gerthoffer WT (2000) Phosphorylation of caldesmon by ERK MAP kinases in smooth muscle. Am $J$ Physiol Cell Physiol 278:C718-C726.

Hemric ME, Lu FW, Shrager R, Carey J, and Chalovich JM (1993) Reversal of caldesmon binding to myosin with calcium-calmodulin or by phosphorylating caldesmon. J Biol Chem 268:15305-15311.

Hermann S, Saarikettu J, Onions J, Hughes K, and Grundström T (1998) Calcium regulation of basic helix-loop-helix transcription factors. Cell Calcium 23:135-142. Herrera AM, McParland BE, Bienkowska A, Tait R, Paré PD, and Seow CY (2005 'Sarcomeres' of smooth muscle: functional characteristics and ultrastructural evidence. J Cell Sci 118:2381-2392.

Herring BP, Lyons GE, Hoggatt AM, and Gallagher PJ (2001) Telokin expression is restricted to smooth muscle tissues during mouse development. Am J Physiol Cell Physiol 280:C12-C21.

Heusch G, Libby P, Gersh B, Yellon D, Böhm M, Lopaschuk G, and Opie L (2014) Cardiovascular remodelling in coronary artery disease and heart failure. Lancet 383:1933-1943.

Hill MA, Falcone JC, and Meininger GA (1990) Evidence for protein kinase C involvement in arteriolar myogenic reactivity. Am J Physiol 259:H1586-H1594.

Hill MA, Zou H, Potocnik SJ, Meininger GA, and Davis MJ (2001) Invited review: arteriolar smooth muscle mechanotransduction: $\mathrm{Ca}(2+)$ signaling pathways underlying myogenic reactivity. J Appl Physiol (1985) 91:973-983.

Hill-Eubanks DC, Werner ME, Heppner TJ, and Nelson MT (2011) Calcium signaling in smooth muscle. Cold Spring Harb Perspect Biol 3:a004549.

Hiltunen MO, Turunen MP, Häkkinen TP, Rutanen J, Hedman M, Mäkinen K, Turunen AM, Aalto-Setälä K, and Ylä-Herttuala S (2002) DNA hypomethylation and methyltransferase expression in atherosclerotic lesions. Vasc Med 7:5-11.

Hiltunen MO and Ylä-Herttuala S (2003) DNA methylation, smooth muscle cells, and atherogenesis. Arterioscler Thromb Vasc Biol 23:1750-1753.

Hodges RR, Horikawa Y, Rios JD, Shatos MA, and Dartt DA (2007) Effect of protein kinase $\mathrm{C}$ and $\mathrm{Ca}(2+)$ on $\mathrm{p} 42 / \mathrm{p} 44 \mathrm{MAPK}$, Pyk2, and Src activation in rat conjunctival goblet cells. Exp Eye Res 85:836-844.

Holdt LM, Sass K, Gäbel G, Bergert H, Thiery J, and Teupser D (2011) Expression of Chr9p21 genes CDKN2B (p15(INK4b)), CDKN2A (p16(INK4a), p14(ARF)) and MTAP in human atherosclerotic plaque. Atherosclerosis 214:264-270.

Holzapfel GA and Ogden RW (2010) Constitutive modelling of arteries. Proceedings of the Royal Society 466:1551-1597.

Hoover WC, Zhang W, Xue Z, Gao H, Chernoff J, Clapp DW, Gunst SJ, and Tepper RS (2012) Inhibition of p21 activated kinase (PAK) reduces airway responsiveness in vivo and in vitro in murine and human airways. PLoS One 7:e42601.

Horowitz A, Clément-Chomienne O, Walsh MP, and Morgan KG (1996a) Epsilonisoenzyme of protein kinase $\mathrm{C}$ induces a $\mathrm{Ca}(2+)$-independent contraction in vascular smooth muscle. Am J Physiol 271:C589-C594. 
Horowitz A, Clément-Chomienne O, Walsh MP, Tao T, Katsuyama H, and Morgan KG (1996b) Effects of calponin on force generation by single smooth muscle cells. Am J Physiol 270:H1858-H1863.

Hou X, Chen J, Luo Y, Liu F, Xu G, and Gao Y (2013) Silencing of STIM1 attenuates hypoxia-induced PASMCs proliferation via inhibition of the SOC/Ca2+/NFAT pathway. Respir Res 14:2.

Huang PL, Huang Z, Mashimo H, Bloch KD, Moskowitz MA, Bevan JA, and Fishman MC (1995) Hypertension in mice lacking the gene for endothelial nitric oxide synthase. Nature 377:239-242.

Huang QQ, Fisher SA, and Brozovich FV (2004) Unzipping the role of myosin light chain phosphatase in smooth muscle cell relaxation. J Biol Chem 279:597-603.

Hudmon A and Schulman H (2002) Neuronal CA2+/calmodulin-dependent protein kinase II: the role of structure and autoregulation in cellular function. Annu Rev Biochem 71:473-510.

Hulvershorn J, Gallant C, Wang CA, Dessy C, and Morgan KG (2001) Calmodulin levels are dynamically regulated in living vascular smooth muscle cells. Am J Physiol Heart Circ Physiol 280:H1422-H1426.

Hutvágner G, McLachlan J, Pasquinelli AE, Bálint E, Tuschl T, and Zamore PD (2001) A cellular function for the RNA-interference enzyme Dicer in the maturation of the let-7 small temporal RNA. Science 293:834-838.

Ichikawa K, Ito M, and Hartshorne DJ (1996) Phosphorylation of the large subunit of myosin phosphatase and inhibition of phosphatase activity. $J$ Biol Chem 271: $4733-4740$

Iino M, Kasai H, and Yamazawa T (1994) Visualization of neural control of intracellular $\mathrm{Ca} 2+$ concentration in single vascular smooth muscle cells in situ. EMBO J 13:5026-5031.

Ikebe $\mathrm{M}$ and Hartshorne DJ (1985) Effects of Ca2+ on the conformation and enzymatic activity of smooth muscle myosin. $J$ Biol Chem 260:13146-13153.

Ikebe M and Morita J (1991) Identification of the sequence of the regulatory light chain required for the phosphorylation-dependent regulation of actomyosin. $J$ Biol Chem 266:21339-21342.

Immink RV, van den Born BJ, van Montfrans GA, Koopmans RP, Karemaker JM, and van Lieshout JJ (2004) Impaired cerebral autoregulation in patients with malignant hypertension. Circulation 110:2241-2245.

Ingber DE (2003) Mechanobiology and diseases of mechanotransduction. Ann Med 35:564-577.

Ingber DE (2006) Cellular mechanotransduction: putting all the pieces together again. FASEB $J$ 20:811-827.

Intengan HD and Schiffrin EL (2000) Structure and mechanical properties of resistance arteries in hypertension: role of adhesion molecules and extracellular matrix determinants. Hypertension 36:312-318.

Ishibe S, Joly D, Zhu X, and Cantley LG (2003) Phosphorylation-dependent paxillinERK association mediates hepatocyte growth factor-stimulated epithelial morphogenesis. Mol Cell 12:1275-1285.

Ito I, Jarajapu YP, Grant MB, and Knot HJ (2007) Characteristics of myogenic tone in the rat ophthalmic artery. Am J Physiol Heart Circ Physiol 292:H360-H368.

Ito M and Hartshorne DJ (1990) Phosphorylation of myosin as a regulatory mechanism in smooth muscle. Prog Clin Biol Res 327:57-72.

Jaenisch R and Bird A (2003) Epigenetic regulation of gene expression: how the genome integrates intrinsic and environmental signals. Nat Genet 33 (Suppl): $245-254$

Jaffe AB and Hall A (2005) Rho GTPases: biochemistry and biology. Annu Rev Cell Dev Biol 21:247-269.

Jaggar JH, Porter VA, Lederer WJ, and Nelson MT (2000) Calcium sparks in smooth muscle. Am J Physiol Cell Physiol 278:C235-C256.

James PR and Nelson-Piercy C (2004) Management of hypertension before, during, and after pregnancy. Heart 90:1499-1504.

Jana SS, Kim KY, Mao J, Kawamoto S, Sellers JR, and Adelstein RS (2009) An alternatively spliced isoform of non-muscle myosin II-C is not regulated by myosin light chain phosphorylation. J Biol Chem 284:11563-11571.

Jarajapu YP and Knot HJ (2005) Relative contribution of Rho kinase and protein kinase $\mathrm{C}$ to myogenic tone in rat cerebral arteries in hypertension. Am J Physio Heart Circ Physiol 289:H1917-H1922.

Jaworowski A, Anderson KI, Arner A, Engström M, Gimona M, Strasser P, and Small JV (1995) Calponin reduces shortening velocity in skinned taenia coli smooth muscle fibres. FEBS Lett 365:167-171.

Je HD, Gallant C, Leavis PC, and Morgan KG (2004) Caveolin-1 regulates contractility in differentiated vascular smooth muscle. Am J Physiol Heart Circ Physiol 286:H91-H98.

Je HD, Gangopadhyay SS, Ashworth TD, and Morgan KG (2001) Calponin is required for agonist-induced signal transduction-evidence from an antisense approach in ferret smooth muscle. J Physiol 537:567-577.

Jensen BL and Skøtt O (1996) Blockade of chloride channels by DIDS stimulates renin release and inhibits contraction of afferent arterioles. Am J Physiol 270 F718-F727.

Ji H, Menini S, Zheng W, Pesce C, Wu X, and Sandberg K (2008) Role of angiotensinconverting enzyme 2 and angiotensin(1-7) in 17beta-oestradiol regulation of renal pathology in renal wrap hypertension in rats. Exp Physiol 93:648-657.

Jiang MJ and Morgan KG (1989) Agonist-specific myosin phosphorylation and intracellular calcium during isometric contractions of arterial smooth muscle. Pflugers Arch 413:637-643.

Jiang Y, Yin H, and Zheng XL (2010) MicroRNA-1 inhibits myocardin-induced contractility of human vascular smooth muscle cells. J Cell Physiol 225:506-511.

Johnson AD, Newton-Cheh C, Chasman DI, Ehret GB, Johnson T, Rose L, Rice K, Verwoert GC, Launer LJ, and Gudnason V, et al.; Cohorts for Heart and Aging Research in Genomic Epidemiology Consortium; Global BPgen Consortium; Women's Genome Health Study (2011) Association of hypertension drug target genes with blood pressure and hypertension in 86,588 individuals. Hypertension 57:903-910.

Johnson JA (2008) Ethnic differences in cardiovascular drug response: potential contribution of pharmacogenetics. Circulation 118:1383-1393.
Johnson JA, Zineh I, Puckett BJ, McGorray SP, Yarandi HN, and Pauly DF (2003) Beta 1-adrenergic receptor polymorphisms and antihypertensive response to metoprolol. Clin Pharmacol Ther 74:44-52.

Johnson RJ, Feig DI, Nakagawa T, Sanchez-Lozada LG, and Rodriguez-Iturbe B (2008) Pathogenesis of essential hypertension: historical paradigms and modern insights. J Hypertens 26:381-391.

Johnson RP, El-Yazbi AF, Takeya K, Walsh EJ, Walsh MP, and Cole WC (2009) Ca2+ sensitization via phosphorylation of myosin phosphatase targeting subunit at threonine- 855 by Rho kinase contributes to the arterial myogenic response. $J$ Physiol 587:2537-2553.

Jones CJ, Kuo L, Davis MJ, and Chilian WM (1995) Regulation of coronary blood flow: coordination of heterogeneous control mechanisms in vascular microdomains. Cardiovasc Res 29:585-596.

Jones JA, Stroud RE, O'Quinn EC, Black LE, Barth JL, Elefteriades JA, Bavaria JE, Gorman JH 3rd, Gorman RC, and Spinale FG, et al. (2011) Selective microRNA suppression in human thoracic aneurysms: relationship of miR-29a to aortic size and proteolytic induction. Circ Cardiovasc Genet 4:605-613.

Joseph BK, Thakali KM, Moore CL, and Rhee SW (2013) Ion channel remodeling in vascular smooth muscle during hypertension: Implications for novel therapeutic approaches. Pharmacol Res 70:126-138.

Jung HS, Billington N, Thirumurugan K, Salzameda B, Cremo CR, Chalovich JM, Chantler PD, and Knight PJ (2011) Role of the tail in the regulated state of myosin 2. J Mol Biol 408:863-878.

Kaiser L, Spickard RC, and Olivier NB (1989) Heart failure depresses endotheliumdependent responses in canine femoral artery. Am J Physiol 256:H962-H967.

Kanchanawong P, Shtengel G, Pasapera AM, Ramko EB, Davidson MW, Hess HF, and Waterman CM (2010) Nanoscale architecture of integrin-based cell adhesions. Nature 468:580-584.

Kandabashi T, Shimokawa H, Miyata K, Kunihiro I, Kawano Y, Fukata Y, Higo T, Egashira K, Takahashi S, and Kaibuchi K, et al. (2000) Inhibition of myosin phosphatase by upregulated rho-kinase plays a key role for coronary artery spasm in a porcine model with interleukin-1beta. Circulation 101:1319-1323.

Kang H, Davis-Dusenbery BN, Nguyen PH, Lal A, Lieberman J, Van Aelst L, Lagna G, and Hata A (2012) Bone morphogenetic protein 4 promotes vascular smooth muscle contractility by activating microRNA-21 (miR-21), which down-regulates expression of family of dedicator of cytokinesis (DOCK) proteins. J Biol Chem 287: $3976-3986$

Karagiannis P, Babu GJ, Periasamy M, and Brozovich FV (2003) The smooth muscle myosin seven amino acid heavy chain insert's kinetic role in the crossbridge cycle for mouse bladder. J Physiol 547:463-473.

Karagiannis P, Babu GJ, Periasamy M, and Brozovich FV (2004) Myosin heavy chain isoform expression regulates shortening velocity in smooth muscle: studies using an SMB KO mouse line. J Muscle Res Cell Motil 25:149-158.

Karaki H (1989) Ca2+ localization and sensitivity in vascular smooth muscle. Trends Pharmacol Sci 10:320-325.

Kargacin GJ (1994) Calcium signaling in restricted diffusion spaces. Biophys $J$ 67: $262-272$

Kargacin GJ, Cooke PH, Abramson SB, and Fay FS (1989) Periodic organization of the contractile apparatus in smooth muscle revealed by the motion of dense bodies in single cells. J Cell Biol 108:1465-1475.

Karim SM, Rhee AY, Given AM, Faulx MD, Hoit BD, and Brozovich FV (2004) Vascular reactivity in heart failure: role of myosin light chain phosphatase. Circ Res 95:612-618.

Karolczak-Bayatti M, Sweeney M, Cheng J, Edey L, Robson SC, Ulrich SM, Treumann A, Taggart MJ, and Europe-Finner GN (2011) Acetylation of heat shock protein 20 (Hsp20) regulates human myometrial activity. J Biol Chem 286:34346-34355.

Kato N, Takeuchi F, Tabara Y, Kelly TN, Go MJ, Sim X, Tay WT, Chen CH, Zhang Y, and Yamamoto K, et al. (2011) Meta-analysis of genome-wide association studies identifies common variants associated with blood pressure variation in east Asians. Nat Genet 43:531-538.

Katoch SS and Moreland RS (1995) Agonist and membrane depolarization induced activation of MAP kinase in the swine carotid artery. Am $J$ Physiol 269: $\mathrm{H} 222-\mathrm{H} 229$

Katz SD, Schwarz M, Yuen J, and LeJemtel TH (1993) Impaired acetylcholine-mediated vasodilation in patients with congestive heart failure. Role of endothelium-derived vasodilating and vasoconstricting factors. Circulation 88:55-61.

Kelley CA, Takahashi M, Yu JH, and Adelstein RS (1993) An insert of seven amino acids confers functional differences between smooth muscle myosins from the intestines and vasculature. J Biol Chem 268:12848-12854.

Khalil RA and Granger JP (2002) Vascular mechanisms of increased arterial pressure in preeclampsia: lessons from animal models. Am J Physiol Regul Integr Comp Physiol 283:R29-R45.

Khalil RA and Morgan KG (1993) PKC-mediated redistribution of mitogen-activated protein kinase during smooth muscle cell activation. Am J Physiol 265:C406-C411.

Khan F, Litchfield SJ, McLaren M, Veale DJ, Littleford RC, and Belch JJ (1997) Ora L-arginine supplementation and cutaneous vascular responses in patients with primary Raynaud's phenomenon. Arthritis Rheum 40:352-357.

Khatri JJ, Joyce KM, Brozovich FV, and Fisher SA (2001) Role of myosin phosphatase isoforms in cGMP-mediated smooth muscle relaxation. $J$ Biol Chem 276 37250-37257.

Khvorova A, Reynolds A, and Jayasena SD (2003) Functional siRNAs and miRNAs exhibit strand bias. Cell 115:209-216.

Kim HR, Gallant C, Leavis PC, Gunst SJ, and Morgan KG (2008a) Cytoskeletal remodeling in differentiated vascular smooth muscle is actin isoform dependent and stimulus dependent. Am J Physiol Cell Physiol 295:C768-C778.

Kim HR, Graceffa P, Ferron F, Gallant C, Boczkowska M, Dominguez R, and Morgan KG (2010) Actin polymerization in differentiated vascular smooth muscle cells requires vasodilator-stimulated phosphoprotein. Am J Physiol Cell Physiol 298: C559-C571. 
Kim I, Je HD, Gallant C, Zhan Q, Riper DV, Badwey JA, Singer HA, and Morgan KG (2000) Ca2+-calmodulin-dependent protein kinase II-dependent activation of contractility in ferret aorta. J Physiol 526:367-374.

Kim SC, Sprung R, Chen Y, Xu Y, Ball H, Pei J, Cheng T, Kho Y, Xiao H, and Xiao L, et al. (2006) Substrate and functional diversity of lysine acetylation revealed by a proteomics survey. Mol Cell 23:607-618.

Kim YS, Immink RV, Stok WJ, Karemaker JM, Secher NH, and van Lieshout JJ (2008b) Dynamic cerebral autoregulatory capacity is affected early in Type 2 diabetes. Clin Sci (Lond) 115:255-262.

Kiss E, Murányi A, Csortos C, Gergely P, Ito M, Hartshorne DJ, and Erdodi F (2002) Integrin-linked kinase phosphorylates the myosin phosphatase target subunit at the inhibitory site in platelet cytoskeleton. Biochem $J$ 365:79-87.

Kitamura K and Yamazaki J (2001) Chloride channels and their functional roles in smooth muscle tone in the vasculature. Jpn J Pharmacol 85:351-357.

Kitazawa T, Eto M, Woodsome TP, and Brautigan DL (2000) Agonists trigger G protein-mediated activation of the CPI-17 inhibitor phosphoprotein of myosin light chain phosphatase to enhance vascular smooth muscle contractility. J Biol Chem 275:9897-9900.

Kitazawa T, Gaylinn BD, Denney GH, and Somlyo AP (1991a) G-protein-mediated $\mathrm{Ca} 2+$ sensitization of smooth muscle contraction through myosin light chain phosphorylation. J Biol Chem 266:1708-1715.

Kitazawa T, Masuo M, and Somlyo AP (1991b) G protein-mediated inhibition of myosin light-chain phosphatase in vascular smooth muscle. Proc Natl Acad Sci USA 88:9307-9310.

Knot HJ and Nelson MT (1998) Regulation of arterial diameter and wall [Ca2+] in cerebral arteries of rat by membrane potential and intravascular pressure. $J$ Physiol 508:199-209.

Knowles HJ, Tian YM, Mole DR, and Harris AL (2004) Novel mechanism of action for hydralazine: induction of hypoxia-inducible factor-1alpha, vascular endothelial growth factor, and angiogenesis by inhibition of prolyl hydroxylases. Circ Res $\mathbf{9 5}$ 162-169

Koga Y and Ikebe M (2005) p116Rip decreases myosin II phosphorylation by activating myosin light chain phosphatase and by inactivating RhoA. J Biol Chem $\mathbf{2 8 0}$ 4983-4991.

Kohn JC, Lampi MC, and Reinhart-King CA (2015) Age-related vascular stiffening: causes and consequences. Front Genet 6:112.

Kolch W (2005) Coordinating ERK/MAPK signalling through scaffolds and inhibitors. Nat Rev Mol Cell Biol 6:827-837.

Kolega J (2003) Asymmetric distribution of myosin IIB in migrating endothelial cells is regulated by a rho-dependent kinase and contributes to tail retraction. Mol Biol Cell 14:4745-4757.

Konik EA, Han YS, and Brozovich FV (2013) The role of pulmonary vascular contractile protein expression in pulmonary arterial hypertension. J Mol Cell Cardiol 65:147-155.

Kontaraki JE, Marketou ME, Zacharis EA, Parthenakis FI, and Vardas PE (2014) Differential expression of vascular smooth muscle-modulating microRNAs in human peripheral blood mononuclear cells: novel targets in essential hypertension. $J$ Hum Hypertens 28:510-516.

Kordowska J, Huang R, and Wang CL (2006) Phosphorylation of caldesmon during smooth muscle contraction and cell migration or proliferation. J Biomed Sci 13: 159-172.

Koshy MC, Mickley D, Bourgiognie J, and Blaufox MD (1977) Physiologic evaluation of a new antihypertensive agent: prazosin HCl. Circulation 55:533-537.

Kothapalli D, Liu SL, Bae YH, Monslow J, Xu T, Hawthorne EA, Byfield FJ, Castagnino P, Rao S, and Rader DJ, et al. (2012) Cardiovascular protection by ApoE and ApoE-HDL linked to suppression of ECM gene expression and arterial stiffening. Cell Reports 2:1259-1271.

Kotlo KU, Hesabi B, and Danziger RS (2011) Implication of microRNAs in atrial natriuretic peptide and nitric oxide signaling in vascular smooth muscle cells. Am $J$ Physiol Cell Physiol 301:C929-C937.

Kouzarides T (2007) Chromatin modifications and their function. Cell 128:693-705.

Kovács M, Thirumurugan K, Knight PJ, and Sellers JR (2007) Load-dependent mechanism of nonmuscle myosin 2. Proc Natl Acad Sci USA 104:9994-9999.

Kovács M, Wang F, Hu A, Zhang Y, and Sellers JR (2003) Functional divergence of human cytoplasmic myosin II: kinetic characterization of the non-muscle IIA isoform. J Biol Chem 278:38132-38140.

Kovtun O, Tillu VA, Ariotti N, Parton RG, and Collins BM (2015) Cavin family proteins and the assembly of caveolae. J Cell Sci 128:1269-1278.

Krennhrubec K, Marshall BL, Hedglin M, Verdin E, and Ulrich SM (2007) Design and evaluation of 'Linkerless' hydroxamic acids as selective HDAC8 inhibitors. Bioorg Med Chem Lett 17:2874-2878.

Kubo SH, Rector TS, Bank AJ, Williams RE, and Heifetz SM (1991) Endotheliumdependent vasodilation is attenuated in patients with heart failure. Circulation $\mathbf{8 4}$ : $1589-1596$.

Kudryavtseva O, Aalkjaer C, and Matchkov VV (2013) Vascular smooth muscle cell phenotype is defined by Ca2+-dependent transcription factors. FEBS $J \mathbf{2 8 0}$ : $5488-5499$

Kumai T, Takeba Y, Matsumoto N, Nakaya S, Tsuzuki Y, Yanagida Y, Hayashi M, and Kobayashi S (2007) Fasudil attenuates sympathetic nervous activity in the adrenal medulla of spontaneously hypertensive rats. Life Sci 81:1193-1198.

Kunichika N, Landsberg JW, Yu Y, Kunichika H, Thistlethwaite PA, Rubin LJ, and Yuan JX (2004) Bosentan inhibits transient receptor potential channel expression in pulmonary vascular myocytes. Am J Respir Crit Care Med 170: 1101-1107.

Lacolley P, Regnault V, Nicoletti A, Li Z, and Michel JB (2012) The vascular smooth muscle cell in arterial pathology: a cell that can take on multiple roles. Cardiovasc Res 95:194-204.

Lamont C, Vainorius E, and Wier WG (2003) Purinergic and adrenergic Ca2+ transients during neurogenic contractions of rat mesenteric small arteries. J Physiol 549:801-808.
Lan B, Deng L, Donovan GM, Chin LY, Syyong HT, Wang L, Zhang J, Pascoe CD, Norris BA, and Liu JC, et al. (2015) Force maintenance and myosin filament assembly regulated by Rho-kinase in airway smooth muscle. Am J Physiol Lung Cell Mol Physiol 308:L1-L10.

Lassen NA (1959) Cerebral blood flow and oxygen consumption in man. Physiol Rev 39:183-238.

Laukkanen MO, Mannermaa S, Hiltunen MO, Aittomäki S, Airenne K, Jänne J, and Ylä-Herttuala S (1999) Local hypomethylation in atherosclerosis found in rabbit ec-sod gene. Arterioscler Thromb Vasc Biol 19:2171-2178.

Laurent S and Boutouyrie P (2007) Recent advances in arterial stiffness and wave reflection in human hypertension. Hypertension 49:1202-1206.

Laurent S, Boutouyrie P, Asmar R, Gautier I, Laloux B, Guize L, Ducimetiere P, and Benetos A (2001) Aortic stiffness is an independent predictor of all-cause and cardiovascular mortality in hypertensive patients. Hypertension 37:1236-1241.

Leblanc N, Ledoux J, Saleh S, Sanguinetti A, Angermann J, O'Driscoll K, Britton F, Perrino BA, and Greenwood IA (2005) Regulation of calcium-activated chloride channels in smooth muscle cells: a complex picture is emerging. Can J Physiol Pharmacol 83:541-556.

Lee E, Hayes DB, Langsetmo K, Sundberg EJ, and Tao TC (2007) Interactions between the leucine-zipper motif of cGMP-dependent protein kinase and the C-terminal region of the targeting subunit of myosin light chain phosphatase. $J$ Mol Biol 373:1198-1212.

Lee RC, Feinbaum RL, and Ambros V (1993) The C. elegans heterochronic gene lin-4 encodes small RNAs with antisense complementarity to lin-14. Cell 75:843-854.

Lee SH, Johnson JD, Walsh MP, Van Lierop JE, Sutherland C, Xu A, Snedden WA, Kosk-Kosicka D, Fromm H, and Narayanan N, et al. (2000a) Differential regulation of $\mathrm{Ca} 2+/$ calmodulin-dependent enzymes by plant calmodulin isoforms and free $\mathrm{Ca} 2$ + concentration. Biochem J 350:299-306.

Lee Y, Ahn C, Han J, Choi H, Kim J, Yim J, Lee J, Provost P, Rådmark O, and Kim S, et al. (2003) The nuclear RNase III Drosha initiates microRNA processing. Nature 425:415-419.

Lee Y, Jeon K, Lee JT, Kim S, and Kim VN (2002) MicroRNA maturation: stepwise processing and subcellular localization. EMBO J 21:4663-4670.

Lee YH, Gallant C, Guo H, Li Y, Wang CA, and Morgan KG (2000b) Regulation of vascular smooth muscle tone by $\mathrm{N}$-terminal region of caldesmon. Possible role of tethering actin to myosin. $J$ Biol Chem 275:3213-3220.

Lee YH, Kim I, Laporte R, Walsh MP, and Morgan KG (1999) Isozyme-specific inhibitors of protein kinase $\mathrm{C}$ translocation: effects on contractility of single permeabilized vascular muscle cells of the ferret. J Physiol 517:709-720.

Leinweber B, Parissenti AM, Gallant C, Gangopadhyay SS, Kirwan-Rhude A, Leavis PC, and Morgan KG (2000) Regulation of protein kinase C by the cytoskeletal protein calponin. J Biol Chem 275:40329-40336.

Leinweber BD, Leavis PC, Grabarek Z, Wang CL, and Morgan KG (1999a) Extracellular regulated kinase (ERK) interaction with actin and the calponin homology (CH) domain of actin-binding proteins. Biochem $J$ 344:117-123.

Leinweber BD, Parissenti A, Leavis PC, and Morgan KG (1999b) Calponin binds the regulatory domain of PKCe and activates kinase activity. abstract Mol Biol Cell 10: $247 \mathrm{a}$.

Lenz S, Lohse P, Seidel U, and Arnold HH (1989) The alkali light chains of human smooth and nonmuscle myosins are encoded by a single gene. Tissue-specific expression by alternative splicing pathways. J Biol Chem 264:9009-9015.

Leung A, Trac C, Jin W, Lanting L, Akbany A, Sætrom P, Schones DE, and Natarajan R (2013) Novel long noncoding RNAs are regulated by angiotensin II in vascular smooth muscle cells. Circ Res 113:266-278.

Levine B, Kalman J, Mayer L, Fillit HM, and Packer M (1990) Elevated circulating levels of tumor necrosis factor in severe chronic heart failure. $N$ Engl J Med 323: $236-241$.

Lewis BP, Burge CB, and Bartel DP (2005) Conserved seed pairing, often flanked by adenosines, indicates that thousands of human genes are microRNA targets. Cell 120:15-20.

Li H, Li W, Gupta AK, Mohler PJ, Anderson ME, and Grumbach IM (2010) Calmodulin kinase II is required for angiotensin II-mediated vascular smooth muscle hypertrophy. Am J Physiol Heart Circ Physiol 298:H688-H698.

Li J, Chen S, Cleary RA, Wang R, Gannon OJ, Seto E, and Tang DD (2014a) Histone deacetylase 8 regulates cortactin deacetylation and contraction in smooth muscle tissues. Am J Physiol Cell Physiol 307:C288-C295.

Li N, Zheng L, Lin P, Danielpour D, Pan Z, and Ma J (2008) Overexpression of Bax induces down-regulation of store-operated calcium entry in prostate cancer cells. $J$ Cell Physiol 216:172-179.

Li SS, Ran YJ, Zhang DD, Li SZ, and Zhu D (2014b) MicroRNA-190 regulates hypoxic pulmonary vasoconstriction by targeting a voltage-gated $\mathrm{K}^{+}$channel in arterial smooth muscle cells. J Cell Biochem 115:1196-1205.

Li SS, Wu Y, Jin X, and Jiang C (2015) The SUR2B subunit of rat vascular KATP channel is targeted by miR-9a-3p induced by prolonged exposure to methylglyoxal. Am J Physiol Cell Physiol 308:C139-C145.

Li Y, Gallant C, Malek S, and Morgan KG (2007) Focal adhesion signaling is required for myometrial ERK activation and contractile phenotype switch before labor. $J$ Cell Biochem 100:129-140.

Li Y, Je HD, Malek S, and Morgan KG (2003) ERK1/2-mediated phosphorylation of myometrial caldesmon during pregnancy and labor. Am J Physiol Regul Integr Comp Physiol 284:R192-R199.

Li Y, Je HD, Malek S, and Morgan KG (2004) Role of ERK1/2 in uterine contractility and preterm labor in rats. Am $J$ Physiol Regul Integr Comp Physiol 287: R328-R335.

Li Y, Reznichenko M, Tribe RM, Hess PE, Taggart M, Kim H, DeGnore JP, Gangopadhyay S, and Morgan KG (2009) Stretch activates human myometrium via ERK, caldesmon and focal adhesion signaling. PLoS One 4:e7489.

Li Y, Yan J, Wu C, Wang Z, Yuan W, and Wang D (2014c) CD137-CD137L interaction regulates atherosclerosis via cyclophilin A in apolipoprotein E-deficient mice. PLoS One 9:e88563. 
Liang R, Bates DJ, and Wang E (2009) Epigenetic control of microRNA expression and aging. Curr Genomics 10:184-193.

Liao YC, Liu PY, Lin HF, Lin WY, Liao JK, and Juo SH (2015) Two functional polymorphisms of ROCK2 enhance arterial stiffening through inhibiting its activity and expression. J Mol Cell Cardiol 79:180-186.

Lifton RP, Gharavi AG, and Geller DS (2001) Molecular mechanisms of human hypertension. Cell 104:545-556.

Lin MJ, Leung GP, Zhang WM, Yang XR, Yip KP, Tse CM, and Sham JS (2004) Chronic hypoxia-induced upregulation of store-operated and receptor-operated $\mathrm{Ca} 2+$ channels in pulmonary arterial smooth muscle cells: a novel mechanism of hypoxic pulmonary hypertension. Circ Res 95:496-505.

Lincoln TM (1989) Cyclic GMP and mechanisms of vasodilation. Pharmacol Ther 41: 479-502

Lincoln TM, Dey N, and Sellak H (2001) Invited review: cGMP-dependent protein kinase signaling mechanisms in smooth muscle: from the regulation of tone to gene expression. J Appl Physiol 91:1421-1430.

Lindheimer MD (1993) Hypertension in pregnancy. Hypertension 22:127-137.

Ling H, Fabbri M, and Calin GA (2013) MicroRNAs and other non-coding RNAs as targets for anticancer drug development. Nat Rev Drug Discov 12:847-865.

Lisman J, Schulman H, and Cline H (2002) The molecular basis of CaMKII function in synaptic and behavioural memory. Nat Rev Neurosci 3:175-190.

Liu JC, Rottler J, Wang L, Zhang J, Pascoe CD, Lan B, Norris BA, Herrera AM, Paré PD, and Seow CY (2013) Myosin filaments in smooth muscle cells do not have a constant length. J Physiol 591:5867-5878.

Liu L and Pilch PF (2008) A critical role of cavin (polymerase I and transcript release factor) in caveolae formation and organization. J Biol Chem 283:4314-4322.

Liu X, Cheng Y, Chen X, Yang J, Xu L, and Zhang C (2011) MicroRNA-31 regulated by the extracellular regulated kinase is involved in vascular smooth muscle cell growth via large tumor suppressor homolog 2. J Biol Chem 286:42371-42380.

Liu Z, Zhang C, Dronadula N, Li Q, and Rao GN (2005) Blockade of nuclear factor of activated T cells activation signaling suppresses balloon injury-induced neointima formation in a rat carotid artery model. J Biol Chem 280:14700-14708.

Löfgren M, Ekblad E, Morano I, and Arner A (2003) Nonmuscle myosin motor of smooth muscle. J Gen Physiol 121:301-310.

Löhn M, Plettenburg O, Ivashchenko Y, Kannt A, Hofmeister A, Kadereit D, Schaefer M, Linz W, Kohlmann M, and Herbert JM, et al. (2009) Pharmacological characterization of SAR407899, a novel rho-kinase inhibitor. Hypertension 54:676-683.

Loirand G and Pacaud P (2014) Involvement of Rho GTPases and their regulators in the pathogenesis of hypertension. Small GTPases 5:1-10.

Lorenzen JM, Martino F, and Thum T (2012) Epigenetic modifications in cardiovascular disease. Basic Res Cardiol 107:245.

Lu KK, Armstrong SE, Ginnan R, and Singer HA (2005) Adhesion-dependent activation of CaMKII and regulation of ERK activation in vascular smooth muscle. Am J Physiol Cell Physiol 289:C1343-C1350.

Lu Y, Zhang H, Gokina N, Mandala M, Sato O, Ikebe M, Osol G, and Fisher SA (2008) Uterine artery myosin phosphatase isoform switching and increased sensitivity to SNP in a rat L-NAME model of hypertension of pregnancy. Am J Physiol Cell Physiol 294:C564-C571.

Lundby A, Lage K, Weinert BT, Bekker-Jensen DB, Secher A, Skovgaard T, Kelstrup CD, Dmytriyev A, Choudhary C, and Lundby C, et al. (2012) Proteomic analysis of lysine acetylation sites in rat tissues reveals organ specificity and subcellular patterns. Cell Reports 2:419-431.

Luo H, Wu Z, Tremblay J, Thorin E, Peng J, Lavoie JL, Hu B, Stoyanova E, Cloutier G, and Qi S, et al. (2012) Receptor tyrosine kinase Ephb6 regulates vascular smooth muscle contractility and modulates blood pressure in concert with sex hormones. J Biol Chem 287:6819-6829.

MacDonald JA, Borman MA, Murányi A, Somlyo AV, Hartshorne DJ, and Haystead TA (2001a) Identification of the endogenous smooth muscle myosin phosphataseassociated kinase. Proc Natl Acad Sci USA 98:2419-2424.

MacDonald JA, Eto M, Borman MA, Brautigan DL, and Haystead TA (2001b) Dual Ser and Thr phosphorylation of CPI-17, an inhibitor of myosin phosphatase, by MYPT-associated kinase. FEBS Lett 493:91-94.

Maegdefessel L, Azuma J, Toh R, Merk DR, Deng A, Chin JT, Raaz U, Schoelmerich AM, Raiesdana A, and Leeper NJ, et al. (2012) Inhibition of microRNA-29b reduces murine abdominal aortic aneurysm development. J Clin Invest 122:497-506.

Maegdefessel L, Rayner KJ, and Leeper NJ (2015a) MicroRNA regulation of vascular smooth muscle function and phenotype: early career committee contribution. Arterioscler Thromb Vasc Biol 35:2-6.

Maegdefessel L, Spin JM, Raaz U, Eken SM, Toh R, Azuma J, Adam M, Nakagami F, Heymann HM, and Chernogubova E, et al. (2015b) Erratum: miR-24 limits aortic vascular inflammation and murine abdominal aneurysm development. Nat Commun 6:6506.

Malmqvist U and Arner A (1991) Correlation between isoform composition of the 17 $\mathrm{kDa}$ myosin light chain and maximal shortening velocity in smooth muscle. Pflugers Arch 418:523-530.

Manabe I and Owens GK (2001) Recruitment of serum response factor and hyperacetylation of histones at smooth muscle-specific regulatory regions during differentiation of a novel P19-derived in vitro smooth muscle differentiation system. Circ Res 88:1127-1134.

Man in't Veld AJ, Van den Meiracker AH, and Schalekamp MA (1988) Do betablockers really increase peripheral vascular resistance? Review of the literature and new observations under basal conditions. Am J Hypertens 1:91-96.

Mann DL (2002) Angiotensin II as an inflammatory mediator: evolving concepts in the role of the renin angiotensin system in the failing heart. Cardiovasc Drugs Ther 16:7-9.

Marganski WA, Gangopadhyay SS, Je HD, Gallant C, and Morgan KG (2005) Targeting of a novel $\mathrm{Ca}+2 /$ calmodulin-dependent protein kinase II is essential for extracellular signal-regulated kinase-mediated signaling in differentiated smooth muscle cells. Circ Res 97:541-549.

Marques FZ, Booth SA, and Charchar FJ (2015) The emerging role of non-coding RNA in essential hypertension and blood pressure regulation. $J$ Hum Hypertens 29:459-467.
Marques FZ, Campain AE, Tomaszewski M, Zukowska-Szczechowska E, Yang YH, Charchar FJ, and Morris BJ (2011) Gene expression profiling reveals renin mRNA overexpression in human hypertensive kidneys and a role for microRNAs. Hypertension 58:1093-1098.

Martinez-Lemus LA, Crow T, Davis MJ, and Meininger GA (2005) alphavbeta3- and alpha5beta1-integrin blockade inhibits myogenic constriction of skeletal muscle resistance arterioles. Am J Physiol Heart Circ Physiol 289:H322-H329.

Martinez-Lemus LA, Wu X, Wilson E, Hill MA, Davis GE, Davis MJ, and Meininger GA (2003) Integrins as unique receptors for vascular control. J Vasc Res 40: 211-233.

Masumoto A, Hirooka Y, Shimokawa H, Hironaga K, Setoguchi S, and Takeshita A (2001) Possible involvement of Rho-kinase in the pathogenesis of hypertension in humans. Hypertension 38:1307-1310.

Matchkov VV, Boedtkjer DM, and Aalkjaer C (2015) The role of $\mathrm{Ca}(2+)$ activated Cl(-) channels in blood pressure control. Curr Opin Pharmacol 21:127-137.

Matchkov VV, Kudryavtseva O, and Aalkjaer C (2012) Intracellular $\mathrm{Ca}^{2+}$ signalling and phenotype of vascular smooth muscle cells. Basic Clin Pharmacol Toxicol 110:42-48.

Matchkov VV, Secher Dam V, Bødtkjer DM, and Aalkjær C (2013) Transport and function of chloride in vascular smooth muscles. J Vasc Res 50:69-87.

Mattace-Raso FUS, van der Cammen TJM, Hofman A, van Popele NM, Bos ML, Schalekamp MADH, Asmar R, Reneman RS, Hoeks APG, and Breteler MMB, et al. (2006) Arterial stiffness and risk of coronary heart disease and stroke: the Rotterdam Study. Circulation 113:657-663.

Mattagajasingh I, Kim CS, Naqvi A, Yamamori T, Hoffman TA, Jung SB, DeRicco J, Kasuno K, and Irani K (2007) SIRT1 promotes endothelium-dependent vascular relaxation by activating endothelial nitric oxide synthase. Proc Natl Acad Sci USA 104:14855-14860.

Matthew JD, Khromov AS, McDuffie MJ, Somlyo AV, Somlyo AP, Taniguchi S, and Takahashi K (2000) Contractile properties and proteins of smooth muscles of a calponin knockout mouse. J Physiol 529:811-824.

Mattick JS and Makunin IV (2006) Non-coding RNA. Hum Mol Genet 15:R17-R29.

McDonald OG and Owens GK (2007) Programming smooth muscle plasticity with chromatin dynamics. Circ Res 100:1428-1441.

McDonald OG, Wamhoff BR, Hoofnagle MH, and Owens GK (2006) Control of SRF binding to CArG box chromatin regulates smooth muscle gene expression in vivo. $J$ Clin Invest 116:36-48.

McFawn PK, Shen L, Vincent SG, Mak A, Van Eyk JE, and Fisher JT (2003) Calcium-independent contraction and sensitization of airway smooth muscle by p21-activated protein kinase. Am J Physiol Lung Cell Mol Physiol 284:L863-L870.

McGoon M, Gutterman D, Steen V, Barst R, McCrory DC, Fortin TA, and Loyd JE American College of Chest Physicians (2004) Screening, early detection, and diagnosis of pulmonary arterial hypertension: ACCP evidence-based clinical practice guidelines. Chest 126(1, Suppl)14S-34S.

McKay MM and Morrison DK (2007) Integrating signals from RTKs to ERK/MAPK. Oncogene 26:3113-3121.

McLendon JM, Joshi SR, Sparks J, Matar M, Fewell JG, Abe K, Oka M, McMurtry IF, and Gerthoffer WT (2015) Lipid nanoparticle delivery of a microRNA-145 inhibitor improves experimental pulmonary hypertension. J Control Release 210: $67-75$

Mehta PK and Griendling KK (2007) Angiotensin II cell signaling: physiological and pathological effects in the cardiovascular system. Am J Physiol Cell Physiol 292 : C82-C97.

Menice CB, Hulvershorn J, Adam LP, Wang CA, and Morgan KG (1997) Calponin and mitogen-activated protein kinase signaling in differentiated vascular smooth muscle. J Biol Chem 272:25157-25161.

Mercado J, Baylie R, Navedo MF, Yuan C, Scott JD, Nelson MT, Brayden JE, and Santana LF (2014) Local control of TRPV4 channels by AKAP150-targeted PKC in arterial smooth muscle. $J$ Gen Physiol 143:559-575.

Mercer TR, Dinger ME, and Mattick JS (2009) Long non-coding RNAs: insights into functions. Nat Rev Genet 10:155-159.

Merk DR, Chin JT, Dake BA, Maegdefessel L, Miller MO, Kimura N, Tsao PS, Iosef C, Berry GJ, and Mohr FW, et al. (2012) miR-29b participates in early aneurysm development in Marfan syndrome. Circ Res 110:312-324.

Merlet E, Atassi F, Motiani RK, Mougenot N, Jacquet A, Nadaud S, Capiod T, Trebak M, Lompré AM, and Marchand A (2013) miR-424/322 regulates vascular smooth muscle cell phenotype and neointimal formation in the rat. Cardiovasc Res 98: $458-468$

Miano JM and Long X (2015) The short and long of noncoding sequences in the control of vascular cell phenotypes. Cell Mol Life Sci 72:3457-3488.

Michael SK, Surks HK, Wang Y, Zhu Y, Blanton R, Jamnongjit M, Aronovitz M, Baur W, Ohtani K, and Wilkerson MK, et al. (2008) High blood pressure arising from a defect in vascular function. Proc Natl Acad Sci USA 105:6702-6707.

Michan S and Sinclair D (2007) Sirtuins in mammals: insights into their biological function. Biochem J 404:1-13.

Michishita E, McCord RA, Berber E, Kioi M, Padilla-Nash H, Damian M, Cheung P, Kusumoto R, Kawahara TL, and Barrett JC, et al. (2008) SIRT6 is a histone H3 lysine 9 deacetylase that modulates telomeric chromatin. Nature 452:492-496.

Miller ED Jr, Ackerly JA, Vaughan ED Jr, Peach MJ, and Epstein RM (1977) The renin-angiotensin system during controlled hypotension with sodium nitroprusside. Anesthesiology 47:257-262.

Mills RD, Mita M, Nakagawa J, Shoji M, Sutherland C, and Walsh MP (2015) A role for the tyrosine kinase Pyk2 in depolarization-induced contraction of vascular smooth muscle. $J$ Biol Chem 290:8677-8692.

Milton DL, Schneck AN, Ziech DA, Ba M, Facemyer KC, Halayko AJ, Baker JE, Gerthoffer WT, and Cremo CR (2011) Direct evidence for functional smooth muscle myosin II in the $10 \mathrm{~S}$ self-inhibited monomeric conformation in airway smooth muscle cells. Proc Natl Acad Sci USA 108:1421-1426.

Min J, Reznichenko M, Poythress RH, Gallant CM, Vetterkind S, Li Y, and Morgan KG (2012) Src modulates contractile vascular smooth muscle function via regulation of focal adhesions. J Cell Physiol 227:3585-3592. 
Mitchell GF, Guo CY, Benjamin EJ, Larson MG, Keyes MJ, Vita JA, Vasan RS, and Levy D (2007) Cross-sectional correlates of increased aortic stiffness in the community: the Framingham Heart Study. Circulation 115:2628-2636.

Miyazaki R, Ichiki T, Hashimoto T, Inanaga K, Imayama I, Sadoshima J, and Sunagawa K (2008) SIRT1, a longevity gene, downregulates angiotensin II type 1 receptor expression in vascular smooth muscle cells. Arterioscler Thromb Vasc Biol 28:1263-1269.

Morano I, Chai GX, Baltas LG, Lamounier-Zepter V, Lutsch G, Kott M, Haase H, and Bader M (2000) Smooth-muscle contraction without smooth-muscle myosin. Nat Cell Biol 2:371-375.

Moreno-Domínguez A, Colinas O, El-Yazbi A, Walsh EJ, Hill MA, Walsh MP, and Cole WC (2013) Ca2+ sensitization due to myosin light chain phosphatase inhibition and cytoskeletal reorganization in the myogenic response of skeletal muscle resistance arteries. $J$ Physiol 591:1235-1250.

Moreno-Domínguez A, El-Yazbi AF, Zhu HL, Colinas O, Zhong XZ, Walsh EJ, Cole DM, Kargacin GJ, Walsh MP, and Cole WC (2014) Cytoskeletal reorganization evoked by Rho-associated kinase- and protein kinase C-catalyzed phosphorylation of cofilin and heat shock protein 27, respectively, contributes to myogenic constriction of rat cerebral arteries. J Biol Chem 289:20939-20952.

Morgan JP and Morgan KG (1982) Vascular smooth muscle: the first recorded Ca2+ transients. Pflugers Arch 395:75-77.

Morgan KG (2014) The importance of the smooth muscle cytoskeleton to preterm labour. Exp Physiol 99:525-529.

Morris SJ and Shore AC (1996) Skin blood flow responses to the iontophoresis of acetylcholine and sodium nitroprusside in man: possible mechanisms. J Physiol 496:531-542.

Morrison DK and Davis RJ (2003) Regulation of MAP kinase signaling modules by scaffold proteins in mammals. Annu Rev Cell Dev Biol 19:91-118.

Morrison DL, Sanghera JS, Stewart J, Sutherland C, Walsh MP, and Pelech SL (1996) Phosphorylation and activation of smooth muscle myosin light chain kinase by MAP kinase and cyclin-dependent kinase-1. Biochem Cell Biol 74:549-557.

Mukai Y, Shimokawa H, Matoba T, Kandabashi T, Satoh S, Hiroki J, Kaibuchi K, and Takeshita A (2001) Involvement of Rho-kinase in hypertensive vascular disease: a novel therapeutic target in hypertension. FASEB 15:1062-1064.

Mulder J, Ariaens A, van den Boomen D, and Moolenaar WH (2004) p116Rip targets myosin phosphatase to the actin cytoskeleton and is essential for RhoA/ROCK regulated neuritogenesis. Mol Biol Cell 15:5516-5527.

Münzel T, Daiber A, and Mülsch A (2005) Explaining the phenomenon of nitrate tolerance. Circ Res 97:618-628.

Murányi A, Derkach D, Erdodi F, Kiss A, Ito M, and Hartshorne DJ (2005) Phosphorylation of Thr695 and Thr850 on the myosin phosphatase target subunit: inhibitory effects and occurrence in A7r5 cells. FEBS Lett 579:6611-6615.

Murányi A, MacDonald JA, Deng JT, Wilson DP, Haystead TA, Walsh MP, Erdodi F, Kiss E, Wu Y, and Hartshorne DJ (2002) Phosphorylation of the myosin phosphatase target subunit by integrin-linked kinase. Biochem $J$ 366:211-216.

Murphy TV, Spurrell BE, and Hill MA (2001) Tyrosine phosphorylation following alterations in arteriolar intraluminal pressure and wall tension. Am J Physiol Heart Circ Physiol 281:H1047-H1056.

Murphy TV, Spurrell BE, and Hill MA (2002) Cellular signalling in arteriolar myogenic constriction: involvement of tyrosine phosphorylation pathways. Clin Exp Pharmacol Physiol 29:612-619.

Nabeshima Y, Nabeshima Y, Nonomura Y, and Fujii-Kuriyama Y (1987) Nonmuscle and smooth muscle myosin light chain mRNAs are generated from a single gene by the tissue-specific alternative RNA splicing. J Biol Chem 262:10608-10612.

Nagai R, Kuro-o M, Babij P, and Periasamy M (1989) Identification of two types of smooth muscle myosin heavy chain isoforms by cDNA cloning and immunoblot analysis. J Biol Chem 264:9734-9737.

Nagai R, Larson DM, and Periasamy M (1988) Characterization of a mammalian smooth muscle myosin heavy chain cDNA clone and its expression in various smooth muscle types. Proc Natl Acad Sci USA 85:1047-1051.

Nakamura K, Koga Y, Sakai H, Homma K, and Ikebe M (2007) cGMP-dependent relaxation of smooth muscle is coupled with the change in the phosphorylation of myosin phosphatase. Circ Res 101:712-722.

Navedo MF and Amberg GC (2013) Local regulation of L-type $\mathrm{Ca}^{2+}$ channel sparklets in arterial smooth muscle. Microcirculation 20:290-298.

Navedo MF, Amberg GC, Votaw VS, and Santana LF (2005) Constitutively active L-type Ca2+ channels. Proc Natl Acad Sci USA 102:11112-11117.

Navedo MF, Cheng EP, Yuan C, Votaw S, Molkentin JD, Scott JD, and Santana LF (2010) Increased coupled gating of L-type Ca2+ channels during hypertension and Timothy syndrome. Circ Res 106:748-756.

Navedo MF, Nieves-Cintrón M, Amberg GC, Yuan C, Votaw VS, Lederer WJ, McKnight GS, and Santana LF (2008) AKAP150 is required for stuttering persistent Ca2+ sparklets and angiotensin II-induced hypertension. Circ Res 102:e1-e11.

Nelson MT and Bonev AD (2004) The beta1 subunit of the Ca2+-sensitive K+ channel protects against hypertension. $J$ Clin Invest 113:955-957.

Nelson MT, Cheng H, Rubart M, Santana LF, Bonev AD, Knot HJ, and Lederer WJ (1995) Relaxation of arterial smooth muscle by calcium sparks. Science 270:633-637.

Nelson MT, Patlak JB, Worley JF, and Standen NB (1990) Calcium channels, potassium channels, and voltage dependence of arterial smooth muscle tone. Am J Physiol 259:C3-C18.

Nguyen DHD, Catling AD, Webb DJ, Sankovic M, Walker LA, Somlyo AV, Weber MJ, and Gonias SL (1999) Myosin light chain kinase functions downstream of Ras/ERK to promote migration of urokinase-type plasminogen activator-stimulated cells in an integrin-selective manner. $J$ Cell Biol 146:149-164.

Nickenig G and Harrison DG (2002) The AT(1)-type angiotensin receptor in oxidative stress and atherogenesis: part I: oxidative stress and atherogenesis. Circulation 105:393-396.

Nieves-Cintrón M, Amberg GC, Navedo MF, Molkentin JD, and Santana LF (2008) The control of $\mathrm{Ca} 2+$ influx and NFATc3 signaling in arterial smooth muscle during hypertension. Proc Natl Acad Sci USA 105:15623-15628.
Nieves-Cintrón M, Amberg GC, Nichols CB, Molkentin JD, and Santana LF (2007) Activation of NFATc3 down-regulates the beta1 subunit of large conductance, calcium-activated $\mathrm{K}+$ channels in arterial smooth muscle and contributes to hypertension. J Biol Chem 282:3231-3240.

Nilsson LM, Nilsson-Ohman J, Zetterqvist AV, and Gomez MF (2008) Nuclear factor of activated T-cells transcription factors in the vasculature: the good guys or the bad guys? Curr Opin Lipidol 19:483-490.

Nixon GF, Iizuka K, Haystead CM, Haystead TA, Somlyo AP, and Somlyo AV (1995) Phosphorylation of caldesmon by mitogen-activated protein kinase with no effect on $\mathrm{Ca} 2+$ sensitivity in rabbit smooth muscle. J Physiol 487:283-289.

Noma K, Goto C, Nishioka K, Jitsuiki D, Umemura T, Ueda K, Kimura M, Nakagawa K, Oshima T, and Chayama K, et al. (2007) Roles of rho-associated kinase and oxidative stress in the pathogenesis of aortic stiffness. J Am Coll Cardiol 49:698-705.

Norata GD, Pinna C, Zappella F, Elia L, Sala A, Condorelli G, and Catapano AL (2012) MicroRNA 143-145 deficiency impairs vascular function. Int J Immunopathol Pharmacol 25:467-474.

North AJ, Gimona M, Cross RA, and Small JV (1994a) Calponin is localised in both the contractile apparatus and the cytoskeleton of smooth muscle cells. J Cell Sci 107:437-444.

North AJ, Gimona M, Lando Z, and Small JV (1994b) Actin isoform compartments in chicken gizzard smooth muscle cells. J Cell Sci 107:445-455.

Obara K, Szymanski PT, Tao T, and Paul RJ (1996) Effects of calponin on isometric force and shortening velocity in permeabilized taenia coli smooth muscle. Am $J$ Physiol 270:C481-C487.

Ohanian J, Gatfield KM, Ward DT, and Ohanian V (2005) Evidence for a functional calcium-sensing receptor that modulates myogenic tone in rat subcutaneous small arteries. Am J Physiol Heart Circ Physiol 288:H1756-H1762.

Ohanian J, Pieri M, and Ohanian V (2014) Non-receptor tyrosine kinases and the actin cytoskeleton in contractile vascular smooth muscle. J Physiol 593:3807-3814. Oka M, Homma N, Taraseviciene-Stewart L, Morris KG, Kraskauskas D, Burns N, Voelkel NF, and McMurtry IF (2007) Rho kinase-mediated vasoconstriction is important in severe occlusive pulmonary arterial hypertension in rats. Circ Res 100:923-929.

Oliveria SF, Dell'Acqua ML, and Sather WA (2007) AKAP79/150 anchoring of calcineurin controls neuronal L-type $\mathrm{Ca} 2+$ channel activity and nuclear signaling Neuron 55:261-275.

Oparil S, Zaman MA, and Calhoun DA (2003) Pathogenesis of hypertension. Ann Intern Med 139:761-776.

Opazo Saez A, Zhang W, Wu Y, Turner CE, Tang DD, and Gunst SJ (2004) Tension development during contractile stimulation of smooth muscle requires recruitment of paxillin and vinculin to the membrane. Am J Physiol Cell Physiol 286: C433-C447.

O'Rourke M (1990) Arterial stiffness, systolic blood pressure, and logical treatment of arterial hypertension. Hypertension 15:339-347.

Osol G, Brekke JF, McElroy-Yaggy K, and Gokina NI (2002) Myogenic tone, reactivity, and forced dilatation: a three-phase model of in vitro arterial myogenic behavior. Am J Physiol Heart Circ Physiol 283:H2260-H2267.

Packer CS, Roepke JE, Oberlies NH, and Rhoades RA (1998) Myosin isoform shifts and decreased reactivity in hypoxia-induced hypertensive pulmonary arteria muscle. Am J Physiol 274:L775-L785.

Padmanabhan S, Newton-Cheh C, and Dominiczak AF (2012) Genetic basis of blood pressure and hypertension. Trends Genet 28:397-408.

Palma-Flores C, Ramírez-Sánchez I, Rosas-Vargas H, Canto P, and Coral-Vázquez RM (2014) Description of a utrophin associated protein complex in lipid raft domains of human artery smooth muscle cells. Biochim Biophys Acta 1838:1047-1054.

Park SY, Park SU, and Sohn UD (2009) Regulators involved in the electrically stimulated response of feline esophageal smooth muscle. Pharmacology 84: $346-355$

Parker CA, Takahashi K, Tang JX, Tao T, and Morgan KG (1998) Cytoskeletal targeting of calponin in differentiated, contractile smooth muscle cells of the ferret. $J$ Physiol 508:187-198.

Parker CA Takahashi K, Tao T, and Morgan KG (1994) Agonist-induced redistribution of calponin in contractile vascular smooth muscle cells. Am J Physio 267:C1262-C1270.

Parris JR, Cobban HJ, Littlejohn AF, MacEwan DJ, and Nixon GF (1999) Tumour necrosis factor-alpha activates a calcium sensitization pathway in guinea-pig bronchial smooth muscle. J Physiol 518:561-569.

Pasquale EB (2008) Eph-ephrin bidirectional signaling in physiology and disease Cell 133:38-52.

Patchell VB, Vorotnikov AV, Gao Y, Low DG, Evans JS, Fattoum A, El-Mezgueldi M, Marston SB, and Levine BA (2002) Phosphorylation of the minimal inhibitory region at the $\mathrm{C}$-terminus of caldesmon alters its structural and actin binding properties. Biochim Biophys Acta 1596:121-130.

Paul RJ, Bowman PS, Johnson J, and Martin AF (2007) Effects of sex and estrogen on myosin $\mathrm{COOH}$-terminal isoforms and contractility in rat aorta. Am J Physiol Regul Integr Comp Physiol 292:R751-R757.

Pavalko FM, Adam LP, Wu MF, Walker TL, and Gunst SJ (1995) Phosphorylation of dense-plaque proteins talin and paxillin during tracheal smooth muscle contraction. Am J Physiol 268:C563-C571.

Payne MC, Zhang HY, Prosdocimo T, Joyce KM, Koga Y, Ikebe M, and Fisher SA (2006) Myosin phosphatase isoform switching in vascular smooth muscle development. J Mol Cell Cardiol 40:274-282.

Payne MC, Zhang HY, Shirasawa Y, Koga Y, Ikebe M, Benoit JN, and Fisher SA (2004) Dynamic changes in expression of myosin phosphatase in a model of portal hypertension. Am J Physiol Heart Circ Physiol 286:H1801-H1810.

Peng H, Matchkov V, Ivarsen A, Aalkjaer C, and Nilsson H (2001) Hypothesis for the initiation of vasomotion. Circ Res 88:810-815.

Pérez GJ, Bonev AD, and Nelson MT (2001) Micromolar Ca(2+) from sparks activates $\mathrm{Ca}(2+)$-sensitive $\mathrm{K}(+)$ channels in rat cerebral artery smooth muscle. Am J Physiol Cell Physiol 281:C1769-C1775. 
Perrin BJ and Ervasti JM (2010) The actin gene family: function follows isoform. Cytoskeleton (Hoboken) 67:630-634.

Pesic A, Madden JA, Pesic M, and Rusch NJ (2004) High blood pressure upregulates arterial L-type $\mathrm{Ca} 2+$ channels: is membrane depolarization the signal? Circ Res 94: e97-e104.

Pestonjamasp KN, Pope RK, Wulfkuhle JD, and Luna EJ (1997) Supervillin (p205): A novel membrane-associated, F-actin-binding protein in the villin/gelsolin superfamily. J Cell Biol 139:1255-1269.

Pfeffer MA, Braunwald E, Moyé LA, Basta L, Brown EJ Jr, Cuddy TE, Davis BR, Geltman EM, Goldman S, and Flaker GC, et al. The SAVE Investigators (1992) Effect of captopril on mortality and morbidity in patients with left ventricular dysfunction after myocardial infarction. Results of the survival and ventricular enlargement trial. N Engl J Med 327:669-677.

Pfeifer A, Klatt P, Massberg S, Ny L, Sausbier M, Hirneiss C, Wang GX, Korth M, Aszódi A and Andersson KE, et al. (1998) Defective smooth muscle regulation in cGMP kinase I-deficient mice. EMBO J 17:3045-3051.

Pfister JA, Ma C, Morrison BE, and D'Mello SR (2008) Opposing effects of sirtuins on neuronal survival: SIRT1-mediated neuroprotection is independent of its deacetylase activity. PLoS One 3:e4090.

Pfitzer G (2001) Invited review: regulation of myosin phosphorylation in smooth muscle. J Appl Physiol 91:497-503.

Pitt B, Poole-Wilson PA, Segal R, Martinez FA, Dickstein K, Camm AJ, Konstam MA, Riegger G, Klinger GH, and Neaton J, et al. (2000) Effect of losartan compared with captopril on mortality in patients with symptomatic heart failure: randomised trial-the Losartan Heart Failure Survival Study ELITE II. Lancet 355:1582-1587.

Plüger S, Faulhaber J, Fürstenau M, Löhn M, Waldschütz R, Gollasch M, Haller H, Luft FC, Ehmke H, and Pongs O (2000) Mice with disrupted BK channel beta1 subunit gene feature abnormal $\mathrm{Ca}(2+)$ spark/STOC coupling and elevated blood pressure. Circ Res 87:E53-E60

Poburko D, Kuo KH, Dai J, Lee CH, and van Breemen C (2004) Organellar junctions promote targeted $\mathrm{Ca} 2+$ signaling in smooth muscle: why two membranes are better than one. Trends Pharmacol Sci 25:8-15.

Posern G, Miralles F, Guettler S, and Treisman R (2004) Mutant actins that stabilise F-actin use distinct mechanisms to activate the SRF coactivator MAL. EMBO J 23:3973-3983.

Potier M, Gonzalez JC, Motiani RK, Abdullaev IF, Bisaillon JM, Singer HA, and Trebak M (2009) Evidence for STIM1- and Orai1-dependent store-operated calcium influx through ICRAC in vascular smooth muscle cells: role in proliferation and migration. FASEB $J$ 23:2425-2437.

Poythress RH, Gallant C, Vetterkind S, and Morgan KG (2013) Vasoconstrictorinduced endocytic recycling regulates focal adhesion protein localization and function in vascular smooth muscle. Am J Physiol Cell Physiol 305:C215-C227.

Pratusevich VR, Seow CY, and Ford LE (1995) Plasticity in canine airway smooth muscle. J Gen Physiol 105:73-94.

Puetz S, Lubomirov LT, and Pfitzer G (2009) Regulation of smooth muscle contraction by small GTPases. Physiology (Bethesda) 24:342-356.

Pulver RA, Rose-Curtis P, Roe MW, Wellman GC, and Lounsbury KM (2004) Storeoperated $\mathrm{Ca} 2+$ entry activates the CREB transcription factor in vascular smooth muscle. Circ Res 94:1351-1358.

Qiao YN, He WQ, Chen CP, Zhang CH, Zhao W, Wang P, Zhang L, Wu YZ, Yang X, and Peng YJ, et al. (2014) Myosin phosphatase target subunit 1 (MYPT1) regulates the contraction and relaxation of vascular smooth muscle and maintains blood pressure. J Biol Chem 289:22512-22523.

Qiu H, Zhu Y, Sun Z, Trzeciakowski JP, Gansner M, Depre C, Resuello RR, Natividad FF, Hunter WC, and Genin GM, et al. (2010) Short communication: vascular smooth muscle cell stiffness as a mechanism for increased aortic stiffness with aging. Circ Res 107:615-619.

Qiu P and Li L (2002) Histone acetylation and recruitment of serum responsive factor and CREB-binding protein onto SM22 promoter during SM22 gene expression. Circ Res 90:858-865.

Quevillon-Chéruel S, Janmot C, Nozais M, Lompré AM, and Béchet JJ (2000) Functional regions in the essential light chain of smooth muscle myosin as revealed by the mutagenesis approach. Eur J Biochem 267:6151-6157.

Rahman A, Davis B, Lövdahl C, Hanumaiah VT, Feil R, Brakebusch C, and Arner A (2014) The small GTPase Rac1 is required for smooth muscle contraction. J Physiol 592:915-926.

Ramchandran R, Raghavan A, Geenen D, Sun M, Bach L, Yang Q, and Raj JU (2014) PKG-1 $\alpha$ leucine zipper domain defect increases pulmonary vascular tone: implications in hypoxic pulmonary hypertension. Am J Physiol Lung Cell Mol Physiol 307:L537-L544.

Reho JJ, Zheng X, Asico LD, and Fisher SA (2015) Redox signaling and splicing dependent change in myosin phosphatase underlie early versus late changes in NO vasodilator reserve in a mouse LPS model of sepsis. Am J Physiol Heart Circ Physiol 308:H1039-H1050.

Rembold CM, Tejani AD, Ripley ML, and Han S (2007) Paxillin phosphorylation, actin polymerization, noise temperature, and the sustained phase of swine carotid artery contraction. Am J Physiol Cell Physiol 293:C993-C1002.

Rhee AY, Ogut O, and Brozovich FV (2006) Nonmuscle myosin, force maintenance, and the tonic contractile phenotype in smooth muscle. Pflugers Arch 452:766-774.

Richards CT, Ogut O, and Brozovich FV (2002) Agonist-induced force enhancement: the role of isoforms and phosphorylation of the myosin-targeting subunit of myosin light chain phosphatase. J Biol Chem 277:4422-4427.

Riches K, Alshanwani AR, Warburton P, O'Regan DJ, Ball SG, Wood IC, Turner NA and Porter KE (2014) Elevated expression levels of miR-143/5 in saphenous vein smooth muscle cells from patients with Type 2 diabetes drive persistent changes in phenotype and function. J Mol Cell Cardiol 74:240-250.

Rinn JL and Chang HY (2012) Genome regulation by long noncoding RNAs. Annu Rev Biochem 81:145-166.

Rokolya A and Singer HA (2000) Inhibition of CaM kinase II activation and force maintenance by KN-93 in arterial smooth muscle. Am J Physiol Cell Physiol 278: C537-C545.
Rosendorff C, Black HR, Cannon CP, Gersh BJ, Gore J, Izzo JL Jr, Kaplan NM, O'Connor CM, O'Gara PT, and Oparil S; American Heart Association Council for High Blood Pressure Research; American Heart Association Council on Clinical Cardiology; American Heart Association Council on Epidemiology and Prevention (2007) Treatment of hypertension in the prevention and management of ischemic heart disease: a scientific statement from the American Heart Association Council for High Blood Pressure Research and the Councils on Clinical Cardiology and Epidemiology and Prevention. Circulation 115:2761-2788.

Rosenfeld SS, Xing J, Chen LQ, and Sweeney HL (2003) Myosin IIb is unconventionally conventional. J Biol Chem 278:27449-27455.

Rosenfeld SS, Xing J, Whitaker M, Cheung HC, Brown F, Wells A, Milligan RA, and Sweeney HL (2000) Kinetic and spectroscopic evidence for three actomyosin: ADP states in smooth muscle. J Biol Chem 275:25418-25426.

Ross R (1993) The pathogenesis of atherosclerosis: a perspective for the 1990s. Nature 362:801-809.

Ross R (1999) Atherosclerosis-an inflammatory disease. N Engl J Med 340:115-126. Rovner AS, Fagnant PM, Lowey S, and Trybus KM (2002) The carboxyl-terminal isoforms of smooth muscle myosin heavy chain determine thick filament assembly properties. J Cell Biol 156:113-123.

Rovner AS, Fagnant PM, and Trybus KM (2006) Phosphorylation of a single head of smooth muscle myosin activates the whole molecule. Biochemistry 45:5280-5289. Rüegg JC and Pfitzer G (1985) Modulation of calcium sensitivity in guinea pig taenia coli: skinned fiber studies. Experientia 41:997-1001.

Russell A and Watts S (2000) Vascular reactivity of isolated thoracic aorta of the C57BL/6J mouse. J Pharmacol Exp Ther 294:598-604.

Salzameda B, Facemyer KC, Beck BW, and Cremo CR (2006) The N-terminal lobes of both regulatory light chains interact with the tail domain in the $10 \mathrm{~S}$-inhibited conformation of smooth muscle myosin. J Biol Chem 281:38801-38811.

Sandoval YH, Atef ME, Levesque LO, Li Y, and Anand-Srivastava MB (2014) Endothelin-1 signaling in vascular physiology and pathophysiology. Curr Vasc Pharmacol 12:202-214.

Sandquist JC, Swenson KI, Demali KA, Burridge K, and Means AR (2006) Rho kinase differentially regulates phosphorylation of nonmuscle myosin II isoforms A and B during cell rounding and migration. J Biol Chem 281:35873-35883.

Saphirstein RJ, Gao YZ, Jensen MH, Gallant CM, Vetterkind S, Moore JR, and Morgan KG (2013) The focal adhesion: a regulated component of aortic stiffness. PLoS One 8:e62461.

Saphirstein RJ, Gao YZ, Lin QQ, and Morgan KG (2015) Cortical actin regulation modulates vascular contractility and compliance in veins. $J$ Physiol 593: 3929-3941.

Saphirstein RJ and Morgan KG (2014) The contribution of vascular smooth muscle to aortic stiffness across length scales. Microcirculation 21:201-207.

Satoh S, Kreutz R, Wilm C, Ganten D, and Pfitzer G (1994) Augmented agonistinduced $\mathrm{Ca}(2+)$-sensitization of coronary artery contraction in genetically hypertensive rats. Evidence for altered signal transduction in the coronary smooth muscle cells. J Clin Invest 94:1397-1403.

Schmidt HH, Lohmann SM, and Walter U (1993) The nitric oxide and cGMP signal transduction system: regulation and mechanism of action. Biochim Biophys Acta 1178:153-175.

Schutzer WE, Reed JF, and Mader SL (2005) Decline in caveolin-1 expression and scaffolding of G protein receptor kinase-2 with age in Fischer 344 aortic vascular smooth muscle. Am J Physiol Heart Circ Physiol 288:H2457-H2464.

Schwarz DS, Hutvágner G, Du T, Xu Z, Aronin N, and Zamore PD (2003) Asymmetry in the assembly of the RNAi enzyme complex. Cell 115:199-208.

Seko T, Ito M, Kureishi Y, Okamoto R, Moriki N, Onishi K, Isaka N, Hartshorne DJ, and Nakano T (2003) Activation of RhoA and inhibition of myosin phosphatase as important components in hypertension in vascular smooth muscle. Circ Res 92: 411-418.

Sellers JR and Adelstein RS (1985) The mechanism of regulation of smooth muscle myosin by phosphorylation. Curr Top Cell Regul 27:51-62.

Sentí M, Fernández-Fernández JM, Tomás M, Vázquez E, Elosua R, Marrugat J, and Valverde MA (2005) Protective effect of the KCNMB1 E65K genetic polymorphism against diastolic hypertension in aging women and its relevance to cardiovascular risk. Circ Res 97:1360-1365.

Seow CY (2013) Hill's equation of muscle performance and its hidden insight on molecular mechanisms. J Gen Physiol 142:561-573.

Seow CY (2015) Reply from Chun Y. Seow. J Physiol 593:477-479.

Sharma AK, Zhou GP, Kupferman J, Surks HK, Christensen EN, Chou JJ, Mendelsohn ME, and Rigby AC (2008) Probing the interaction between the coiled coil leucine zipper of cGMP-dependent protein kinase Ialpha and the $\mathrm{C}$ terminus of the myosin binding subunit of the myosin light chain phosphatase. $J$ Biol Chem 283:32860-32869.

Sharma P, Basu S, Mitchell RW, Stelmack GL, Anderson JE, and Halayko AJ (2014) Role of dystrophin in airway smooth muscle phenotype, contraction and lung function. PLoS One 9:e102737.

Shimizu H, Ito M, Miyahara M, Ichikawa K, Okubo S, Konishi T, Naka M, Tanaka T, Hirano K, and Hartshorne DJ, et al. (1994) Characterization of the myosin-binding subunit of smooth muscle myosin phosphatase. J Biol Chem 269:30407-30411.

Shin J and Johnson JA (2007) Pharmacogenetics of beta-blockers. Pharmacotherapy 27:874-887.

Shukla S and Fisher SA (2008) Tra2beta as a novel mediator of vascular smooth muscle diversification. Circ Res 103:485-492.

Siegman MJ (2014) The pathway for force transmission in the rat anococcygeus muscle: a tale of two tendons. Anat Rec (Hoboken) 297:1714-1733.

Sikuler E, Kravetz D, and Groszmann RJ (1985) Evolution of portal hypertension and mechanisms involved in its maintenance in a rat model. Am J Physiol 248:G618-G625.

Singer HA (2012) Ca2+/calmodulin-dependent protein kinase II function in vascular remodelling. $J$ Physiol 590:1349-1356.

Singh DK, Sarkar J, Raghavan A, Reddy SP, and Raj JU (2011) Hypoxia modulates the expression of leucine zipper-positive MYPT1 and its interaction with protein 
kinase G and Rho kinases in pulmonary arterial smooth muscle cells. Pulm Circ 1: $487-498$

Sitbon O, Humbert M, Jagot JL, Taravella O, Fartoukh M, Parent F, Herve P, and Simonneau G (1998) Inhaled nitric oxide as a screening agent for safely identifying responders to oral calcium-channel blockers in primary pulmonary hypertension. Eur Respir $J$ 12:265-270.

Small JV, Fürst DO, and De Mey J (1986) Localization of filamin in smooth muscle. $J$ Cell Biol 102:210-220.

Smolock EM, Trappanese DM, Chang S, Wang T, Titchenell P, and Moreland RS (2009) siRNA-mediated knockdown of h-caldesmon in vascular smooth muscle. Am $J$ Physiol Heart Circ Physiol 297:H1930-H1939.

Sobey CG (2001) Potassium channel function in vascular disease. Arterioscler Thromb Vasc Biol 21:28-38.

Sobue K, Morimoto K, Inui M, Kanda K, and Kakiuchi S (1982) Control of actinmyosin interaction of gizzard smooth muscle by calmodulin and caldesmon-linked flip-flop mechanism. Biomed Res 3:188-196.

Somlyo AP (1997) Signal transduction. Rhomantic interludes raise blood pressure. Nature 389:908-909, 911.

Somlyo AP and Somlyo AV (1994) Signal transduction and regulation in smooth muscle. Nature 372:231-236.

Somlyo AP and Somlyo AV (2003) Ca2+ sensitivity of smooth muscle and nonmuscle myosin II: modulated by G proteins, kinases, and myosin phosphatase. Physiol Rev 83:1325-1358.

Somlyo AP, Wu X, Walker LA, and Somlyo AV (1999) Pharmacomechanical coupling: the role of calcium, G-proteins, kinases and phosphatases. Rev Physiol Biochem Pharmacol 134:201-234.

Somlyo AV (1980) Ultrastructure of vascular smooth muscle, in Handbook of Physiology, (Geiger SR ed) pp 33-67, American Physiological Society, Bethesda, MD.

Somlyo AV (2015) Smooth muscle myosin filament controversy, once again? J Physiol 593: $473-475$

Somlyo AV and Somlyo AP (1968) Electromechanical and pharmacomechanical coupling in vascular smooth muscle. J Pharmacol Exp Ther 159:129-145.

Sorescu D, Szöcs K, and Griendling KK (2001) NAD(P)H oxidases and their relevance to atherosclerosis. Trends Cardiovasc Med 11:124-131.

Sotiropoulos A, Gineitis D, Copeland J, and Treisman R (1999) Signal-regulated activation of serum response factor is mediated by changes in actin dynamics. Cell 98:159-169.

Spin JM, Quertermous T, and Tsao PS (2010) Chromatin remodeling pathways in smooth muscle cell differentiation, and evidence for an integral role for p300. PLoS One 5:e14301.

Spinelli AM, Liu Y, Sun LY, González-Cobos JC, Backs J, Trebak M, and Singer HA (2015) Smooth muscle CaMKII responsiveness and inflammation. Pflugers Arch 467:2541-2554.

Spurrell BE, Murphy TV, and Hill MA (2003) Intraluminal pressure stimulates MAPK phosphorylation in arterioles: temporal dissociation from myogenic contractile response. Am J Physiol Heart Circ Physiol 285:H1764-H1773.

Staiculescu MC, Galiñanes EL, Zhao G, Ulloa U, Jin M, Beig MI, Meininger GA and Martinez-Lemus LA (2013) Prolonged vasoconstriction of resistance arteries involves vascular smooth muscle actin polymerization leading to inward remodelling. Cardiovasc Res 98:428-436.

Stein JJ, Iwuchukwu C, Maier KG, and Gahtan V (2014) Thrombospondin-1-induced vascular smooth muscle cell migration and proliferation are functionally dependent on microRNA-21. Surgery 155:228-233.

Stein S and Matter CM (2011) Protective roles of SIRT1 in atherosclerosis. Cell Cycle 10:640-647.

Stenmark KR, Fagan KA, and Frid MG (2006) Hypoxia-induced pulmonary vascular remodeling: cellular and molecular mechanisms. Circ Res 99:675-691.

Stevenson AS, Gomez MF, Hill-Eubanks DC, and Nelson MT (2001) NFAT4 movement in native smooth muscle. A role for differential $\mathrm{Ca}(2+)$ signaling. J Biol Chem 276:15018-15024.

Stewart M, Needham M, Bankhead P, Gardiner TA, Scholfield CN, Curtis TM, and McGeown JG (2012) Feedback via $\mathrm{Ca}^{2+}$-activated ion channels modulates endothelin 1 signaling in retinal arteriolar smooth muscle. Invest Ophthalmol Vis Sci 53:3059-3066.

Straight AF, Cheung A, Limouze J, Chen I, Westwood NJ, Sellers JR, and Mitchison TJ (2003) Dissecting temporal and spatial control of cytokinesis with a myosin II Inhibitor. Science 299:1743-1747.

Strasser P, Gimona M, Moessler H, Herzog M, and Small JV (1993) Mammalian calponin. Identification and expression of genetic variants. FEBS Lett 330:13-18.

Sun Z, Martinez-Lemus LA, Hill MA, and Meininger GA (2008) Extracellular matrixspecific focal adhesions in vascular smooth muscle produce mechanically active adhesion sites. Am J Physiol Cell Physiol 295:C268-C278.

Surks HK and Mendelsohn ME (2003) Dimerization of cGMP-dependent protein kinase 1a and the myosin-binding subunit of myosin phosphatase: Role of leucine sipper domains. Cell Signal 15:937-944.

Surks HK, Mochizuki N, Kasai Y, Georgescu SP, Tang KM, Ito M, Lincoln TM, and Mendelsohn ME (1999) Regulation of myosin phosphatase by a specific in teraction with cGMP- dependent protein kinase Ialpha. Science 286:1583-1587.

Surks HK, Richards CT, and Mendelsohn ME (2003) Myosin phosphatase-Rho interacting protein. A new member of the myosin phosphatase complex that directly binds RhoA. J Biol Chem 278:51484-51493.

Swärd K, Albinsson S, and Rippe C (2014) Arterial dysfunction but maintained systemic blood pressure in cavin-1-deficient mice. PLoS One 9:e92428.

Swärd K, Dreja K, Susnjar M, Hellstrand P, Hartshorne DJ, and Walsh MP (2000) Inhibition of Rho-associated kinase blocks agonist-induced $\mathrm{Ca} 2+$ sensitization of myosin phosphorylation and force in guinea-pig ileum. J Physiol 522:33-49.

Sweeney HL and Houdusse A (2010) Structural and functional insights into the Myosin motor mechanism. Annu Rev Biophys 39:539-557.

Sweeney M, Yu Y, Platoshyn O, Zhang S, McDaniel SS, and Yuan JX (2002) Inhibition of endogenous TRP1 decreases capacitative Ca2+ entry and attenuates pulmonary artery smooth muscle cell proliferation. Am J Physiol Lung Cell Mol Physiol 283:L144-L155.

Szymanski PT, Dickie R, Rogers R, and Fredberg JJ (2003) Extraction and reconstitution of calponin and consequent contractile ability in permeabilized smooth muscle fibers. Anal Biochem 321:8-21.

Tabas I, García-Cardeña G, and Owens GK (2015) Recent insights into the cellular biology of atherosclerosis. J Cell Biol 209:13-22.

Tajada S, Cidad P, Colinas O, Santana LF, López-López JR, and Pérez-García MT (2013) Down-regulation of CaV1.2 channels during hypertension: how fewer CaV1.2 channels allow more $\mathrm{Ca}(2+)$ into hypertensive arterial smooth muscle. $J$ Physiol 591:6175-6191.

Takahashi K, Hiwada K, and Kokubu T (1988) Vascular smooth muscle calponin. A novel troponin T-like protein. Hypertension 11:620-626

Takahashi K, Yoshimoto R, Fuchibe K, Fujishige A, Mitsui-Saito M, Hori M, Ozaki H, Yamamura H, Awata N, and Taniguchi S, et al. (2000) Regulation of shortening velocity by calponin in intact contracting smooth muscles. Biochem Biophys Res Commun 279:150-157.

Takahashi Y, Watanabe H, Murakami M, Ono K, Munehisa Y, Koyama T, Nobori K, Iijima T, and Ito H (2007) Functional role of stromal interaction molecule 1 (STIM1) in vascular smooth muscle cells. Biochem Biophys Res Commun 361:934-940.

Takeya K, Loutzenhiser K, Shiraishi M, Loutzenhiser R, and Walsh MP (2008) A highly sensitive technique to measure myosin regulatory light chain phosphorylation: the first quantification in renal arterioles. Am J Physiol Renal Physiol 294: F1487-F1492.

Takizawa N, Koga Y, and Ikebe M (2002) Phosphorylation of CPI17 and myosin binding subunit of type 1 protein phosphatase by p21-activated kinase. Biochem Biophys Res Commun 297:773-778.

Tanaka H, Homma K, White HD, Yanagida T, and Ikebe M (2008) Smooth muscle myosin phosphorylated at single head shows sustained mechanical activity. J Biol Chem 283:15611-15618.

Tanaka K, Minami H, Kota M, Kuwamura K, and Kohmura E (2005) Treatment of cerebral vasospasm with intra-arterial fasudil hydrochloride. Neurosurgery $\mathbf{5 6}$ 214-223, discussion 214-223.

Tanaka Y, Meera P, Song M, Knaus HG, and Toro L (1997) Molecular constituents of maxi KCa channels in human coronary smooth muscle: predominant alpha + beta subunit complexes. J Physiol 502:545-557.

Tang DD (2008) Intermediate filaments in smooth muscle. Am J Physiol Cell Physiol 294:C869-C878.

Tang KM, Wang GR, Lu P, Karas RH, Aronovitz M, Heximer SP, Kaltenbronn KM, Blumer KJ, Siderovski DP, and Zhu Y, et al. (2003) Regulator of G-protein signaling-2 mediates vascular smooth muscle relaxation and blood pressure. Nat Med 9:1506-1512.

Tejani AD, Walsh MP, and Rembold CM (2011) Tissue length modulates "stimulated actin polymerization," force augmentation, and the rate of swine carotid arterial contraction. Am J Physiol Cell Physiol 301:C1470-C1478.

Thomas D, Lipp P, Berridge MJ, and Bootman MD (1998) Hormone-evoked elementary $\mathrm{Ca} 2+$ signals are not stereotypic, but reflect activation of different size channel clusters and variable recruitment of channels within a cluster. $J$ Biol Chem 273:27130-27136.

Thompson AM, Wagner R, and Rzucidlo EM (2014) Age-related loss of SirT1 expression results in dysregulated human vascular smooth muscle cell function. Am $J$ Physiol Heart Circ Physiol 307:H533-H541.

Toro L, Wallner M, Meera P, and Tanaka Y (1998) Maxi-K(Ca), a Unique Member of the Voltage-Gated K Channel Superfamily. News Physiol Sci 13:112-117.

Torre-Amione G, Kapadia S, Lee J, Durand JB, Bies RD, Young JB, and Mann DL (1996) Tumor necrosis factor-alpha and tumor necrosis factor receptors in the failing human heart. Circulation 93:704-711.

Tragante V, Barnes MR, Ganesh SK, Lanktree MB, Guo W, Franceschini N, Smith EN, Johnson T, Holmes MV, and Padmanabhan S, et al. (2014) Gene-centric metaanalysis in 87,736 individuals of European ancestry identifies multiple bloodpressure-related loci. Am J Hum Genet 94:349-360.

Trebak M (2012) STIM/Orai signalling complexes in vascular smooth muscle. $J$ Physiol 590:4201-4208.

Trinkle-Mulcahy L, Ichikawa K, Hartshorne DJ, Siegman MJ, and Butler TM (1995) Thiophosphorylation of the $130-\mathrm{kDa}$ subunit is associated with a decreased activity of myosin light chain phosphatase in alpha-toxin-permeabilized smooth muscle. $J$ Biol Chem 270:18191-18194.

Turczyńska KM, Bhattachariya A, Säll J, Göransson O, Swärd K, Hellstrand P, and Albinsson S (2013) Stretch-sensitive down-regulation of the miR-144/451 cluster in vascular smooth muscle and its role in AMP-activated protein kinase signaling. PLoS One 8:e65135.

Turczynska KM, Sadegh MK, Hellstrand P, Swärd K, and Albinsson S (2012) MicroRNAs are essential for stretch-induced vascular smooth muscle contractile differentiation via microRNA (miR)-145-dependent expression of L-type calcium channels. J Biol Chem 287:19199-19206.

Turner ST, Schwartz GL, Chapman AB, and Boerwinkle E (2001) C825T polymorphism of the $\mathrm{G}$ protein beta(3)-subunit and antihypertensive response to a thiazide diuretic. Hypertension 37:739-743.

Uchida S and Dimmeler S (2015) Long noncoding RNAs in cardiovascular diseases. Circ Res 116:737-750.

Uehata M, Ishizaki T, Satoh H, Ono T, Kawahara T, Morishita T, Tamakawa H, Yamagami K, Inui J, and Maekawa M, et al. (1997) Calcium sensitization of smooth muscle mediated by a Rho-associated protein kinase in hypertension. Nature 389:990-994.

Usui T, Okada M, Mizuno W, Oda M, Ide N, Morita T, Hara Y, and Yamawaki H (2012) HDAC4 mediates development of hypertension via vascular inflammation in spontaneous hypertensive rats. Am J Physiol Heart Circ Physiol 302:H1894-H1904.

Valentín A, Humphrey JD, and Holzapfel GA (2011) A multi-layered computational model of coupled elastin degradation, vasoactive dysfunction, and collagenous stiffening in aortic aging. Ann Biomed Eng 39:2027-2045. 
Valenzuela-Fernández A, Cabrero JR, Serrador JM, and Sánchez-Madrid F (2008) HDAC6: a key regulator of cytoskeleton, cell migration and cell-cell interactions. Trends Cell Biol 18:291-297.

Van Eyk JE, Arrell DK, Foster DB, Strauss JD, Heinonen TY, FurmaniakKazmierczak E, Côté GP, and Mak AS (1998) Different molecular mechanisms for Rho family GTPase-dependent, Ca2+-independent contraction of smooth muscle. J Biol Chem 273:23433-23439.

Van Lierop JE, Wilson DP, Davis JP, Tikunova S, Sutherland C, Walsh MP, and Johnson JD (2002) Activation of smooth muscle myosin light chain kinase by calmodulin. Role of LYS(30) and GLY(40). J Biol Chem 277:6550-6558.

van Rooij E and Kauppinen S (2014) Development of microRNA therapeutics is coming of age. EMBO Mol Med 6:851-864.

Veigel C, Molloy JE, Schmitz S, and Kendrick-Jones J (2003) Load-dependent kinetics of force production by smooth muscle myosin measured with optical tweezers. Nat Cell Biol 5:980-986.

Velasco G, Armstrong C, Morrice N, Frame S, and Cohen P (2002) Phosphorylation of the regulatory subunit of smooth muscle protein phosphatase $1 \mathrm{M}$ at Thr850 in duces its dissociation from myosin. FEBS Lett 527:101-104.

Velaz L, Ingraham RH, and Chalovich JM (1990) Dissociation of the effect of caldesmon on the ATPase activity and on the binding of smooth heavy meromyosin to actin by partial digestion of caldesmon. J Biol Chem 265:2929-2934.

Vetterkind S, Lee E, Sundberg E, Poythress RH, Tao TC, Preuss U, and Morgan KG (2010) Par-4: a new activator of myosin phosphatase. Mol Biol Cell 21:1214-1224

Vetterkind S and Morgan KG (2009) The pro-apoptotic protein Par-4 facilitates vascular contractility by cytoskeletal targeting of ZIPK. J Cell Mol Med 13: 887-895

Vetterkind S, Poythress RH, Lin QQ, and Morgan KG (2013) Hierarchical scaffolding of an ERK1/2 activation pathway. Cell Commun Signal 11:65.

Vigetti D, Deleonibus S, Moretto P, Bowen T, Fischer JW, Grandoch M, Oberhuber A, Love DC, Hanover JA, and Cinquetti R, et al. (2014) Natural antisense transcript for hyaluronan synthase 2 (HAS2-AS1) induces transcription of HAS2 via protein O-GlcNAcylation. $J$ Biol Chem 289:28816-28826.

Wagenseil JE and Mecham RP (2012) Elastin in large artery stiffness and hypertension. J Cardiovasc Transl Res 5:264-273.

Wain LV, Verwoert GC, O'Reilly PF, Shi G, Johnson T, Johnson AD, Bochud M, Rice KM, Henneman P, and Smith AV et al.; LifeLines Cohort Study; EchoGen consortium; AortaGen Consortium; CHARGE Consortium Heart Failure Working Group; KidneyGen consortium; CKDGen consortium; Cardiogenics consortium; ; CardioGram (2011) Genome-wide association study identifies six new loci influencing pulse pressure and mean arterial pressure. Nat Genet 43:1005-1011.

Walsh MP, Andrea JE, Allen BG, Clément-Chomienne O, Collins EM, and Morgan KG (1994) Smooth muscle protein kinase C. Can J Physiol Pharmacol 72: 1392-1399

Walsh MP and Cole WC (2013) The role of actin filament dynamics in the myogenic response of cerebral resistance arteries. J Cereb Blood Flow Metab 33:1-12.

Waltregny D, Glénisson W, Tran SL, North BJ, Verdin E, Colige A, and Castronovo V (2005) Histone deacetylase HDAC8 associates with smooth muscle alpha-actin and is essential for smooth muscle cell contractility. FASEB $J$ 19:966-968.

Wang C-LA, Wang L-WC, Xu SA, Lu RC, Saavedra-Alanis V, and Bryan J (1991) Localization of the calmodulin- and the actin-binding sites of caldesmon. $J$ Biol Chem 266:9166-9172.

Wang CL (2008) Caldesmon and the regulation of cytoskeletal functions. Adv Exp Med Biol 644.250-272.

Wang F, Kovacs M, Hu A, Limouze J, Harvey EV, and Sellers JR (2003) Kinetic mechanism of non-muscle myosin IIB: functional adaptations for tension generation and maintenance. J Biol Chem 278:27439-27448.

Wang G, Kwan BC, Lai FM, Choi PC, Chow KM, Li PK, and Szeto CC (2010) Intrarenal expression of microRNAs in patients with IgA nephropathy. Lab Invest 90:98-103.

Wang GR, Zhu Y, Halushka PV, Lincoln TM, and Mendelsohn ME (1998) Mechanism of platelet inhibition by nitric oxide: in vivo phosphorylation of thromboxane re ceptor by cyclic GMP-dependent protein kinase. Proc Natl Acad Sci USA 95: $4888-4893$.

Wang H, Long CL, and Zhang YL (2005) A new ATP-sensitive potassium channel opener reduces blood pressure and reverses cardiovascular remodeling in experimental hypertension. J Pharmacol Exp Ther 312:1326-1333.

Wang R, Li Q, and Tang DD (2006) Role of vimentin in smooth muscle force development. Am J Physiol Cell Physiol 291:C483-C489.

Wang R, Li QF, Anfinogenova Y, and Tang DD (2007) Dissociation of Crk-associated substrate from the vimentin network is regulated by p21-activated kinase on ACh activation of airway smooth muscle. Am J Physiol Lung Cell Mol Physiol 292 L240-L248.

Wang Y, Thorin E, Luo H, Tremblay J, Lavoie JL, Wu Z, Peng J, Qi S, and Wu J (2015) EPHB4 Protein Expression in Vascular Smooth Muscle Cells Regulate Their Contractility, and EPHB4 Deletion Leads to Hypotension in Mice. J Biol Chem 290:14235-14244.

Watson N, Linder ME, Druey KM, Kehrl JH, and Blumer KJ (1996) RGS family members: GTPase-activating proteins for heterotrimeric G-protein alpha-subunits Nature 383:172-175.

Webb TI, Kshatri AS, Large RJ, Akande AM, Roy S, Sergeant GP, McHale NG, Thornbury KD, and Hollywood MA (2015) Molecular mechanisms underlying the effect of the novel BK channel opener GoSlo: involvement of the S4/S5 linker and the S6 segment. Proc Natl Acad Sci USA 112:2064-2069.

Wellman GC and Nelson MT (2003) Signaling between SR and plasmalemma in smooth muscle: sparks and the activation of $\mathrm{Ca} 2+-$ sensitive ion channels. Cell Calcium 34:211-229.

Westcott EB, Goodwin EL, Segal SS, and Jackson WF (2012) Function and expression of ryanodine receptors and inositol 1,4,5-trisphosphate receptors in smooth muscle cells of murine feed arteries and arterioles. J Physiol 590: 1849-1869.
Westcott EB and Jackson WF (2011) Heterogeneous function of ryanodine receptors, but not IP3 receptors, in hamster cremaster muscle feed arteries and arterioles. Am J Physiol Heart Circ Physiol 300:H1616-H1630.

Wickenden AD, Grimwood S, Grant TL, and Todd MH (1991) Comparison of the effects of the $\mathrm{K}(+)$-channel openers cromakalim and minoxidil sulphate on vascular smooth muscle. Br J Pharmacol 103:1148-1152.

Willum-Hansen T, Staessen JA, Torp-Pedersen C, Rasmussen S, Thijs L, Ibsen H, and Jeppesen J (2006) Prognostic value of aortic pulse wave velocity as index of arterial stiffness in the general population. Circulation 113:664-670.

Winder SJ, Allen BG, Clément-Chomienne O, and Walsh MP (1998) Regulation of smooth muscle actin-myosin interaction and force by calponin. Acta Physiol Scand 164:415-426.

Winder SJ, Allen BG, Fraser ED, Kang HM, Kargacin GJ, and Walsh MP (1993) Calponin phosphorylation in vitro and in intact muscle. Biochem $J$ 296:827-836.

Winder SJ and Walsh MP (1990) Smooth muscle calponin. Inhibition of actomyosin MgATPase and regulation by phosphorylation. J Biol Chem 265:10148-10155.

Wirth A, Benyó Z, Lukasova M, Leutgeb B, Wettschureck N, Gorbey S, Orsy P, Horváth B, Maser-Gluth C, and Greiner E, et al. (2008) G12-G13-LARG-mediated signaling in vascular smooth muscle is required for salt-induced hypertension. Nat Med 14:64-68.

Wirth A, Schroeter M, Kock-Hauser C, Manser E, Chalovich JM, De Lanerolle P, and Pfitzer G (2003) Inhibition of contraction and myosin light chain phosphorylation in guinea-pig smooth muscle by p21-activated kinase 1. J Physiol $\mathbf{5 4 9}$ 489-500.

Woodsome TP, Eto M, Everett A, Brautigan DL, and Kitazawa T (2001) Expression of CPI-17 and myosin phosphatase correlates with $\mathrm{Ca}(2+)$ sensitivity of protein kinase $\mathrm{C}$-induced contraction in rabbit smooth muscle. $J$ Physiol 535:553-564.

Wooldridge AA MacDonald JA, Erdodi F, Ma C, Borman MA, Hartshorne DJ, and Haystead TA (2004) Smooth muscle phosphatase is regulated in vivo by exclusion of phosphorylation of threonine 696 of MYPT1 by phosphorylation of Serine 695 in response to cyclic nucleotides. J Biol Chem 279:34496-34504

Worley JF, Quayle JM, Standen NB, and Nelson MT (1991) Regulation of single calcium channels in cerebral arteries by voltage, serotonin, and dihydropyridines. Am J Physiol 261:H1951-H1960.

Wu C, So J, Davis-Dusenbery BN, Qi HH, Bloch DB, Shi Y, Lagna G, and Hata A (2011) Hypoxia potentiates microRNA-mediated gene silencing through posttranslational modification of Argonaute2. Mol Cell Biol 31:4760-4774.

Wu G, Cai J, Han Y, Chen J, Huang ZP, Chen C, Cai Y, Huang H, Yang Y, and Liu Y, et al. (2014) LincRNA-p21 regulates neointima formation, vascular smooth muscle cell proliferation, apoptosis, and atherosclerosis by enhancing p53 activity. Circulation 130:1452-1465.

Wu X, Haystead TA, Nakamoto RK, Somlyo AV, and Somlyo AP (1998) Acceleration of myosin light chain dephosphorylation and relaxation of smooth muscle by telokin. Synergism with cyclic nucleotide-activated kinase. $J$ Biol Chem $\mathbf{2 7 3}$ 11362-11369.

Wu Z, Luo H, Thorin E, Tremblay J, Peng J, Lavoie JL, Wang Y, Qi S, Wu T, and Wu $J$ (2012) Possible role of Efnb1 protein, a ligand of Eph receptor tyrosine kinases, in modulating blood pressure. J Biol Chem 287:15557-15569.

Wu ZY and Benoit JN (1994) Vascular NE responsiveness in portal hypertension: role of portal pressure and portosystemic shunting. Am J Physiol 266: H1162-H1168.

Xiao B, Gu SM, Li MJ, Li J, Tao B, Wang Y, Wang Y, Zuo S, Shen Y, and Yu Y, et al. (2015) Rare SNP rs12731181 in the miR-590-3p target site of the prostaglandin F2 $\alpha$ receptor gene confers risk for essential hypertension in the Han Chinese population. Arterioscler Thromb Vasc Biol 35:1687-1695.

Xin M, Small EM, Sutherland LB, Qi X, McAnally J, Plato CF, Richardson JA, Bassel-Duby R, and Olson EN (2009) MicroRNAs miR-143 and miR-145 modulate cytoskeletal dynamics and responsiveness of smooth muscle cells to injury. Genes Dev 23:2166-2178.

$\mathrm{Xu}$ SZ and Beech DJ (2001) TrpC1 is a membrane-spanning subunit of store-operated $\mathrm{Ca}(2+)$ channels in native vascular smooth muscle cells. Circ Res 88:84-87.

$\mathrm{Xu}$ X, Ha CH, Wong C, Wang W, Hausser A, Pfizenmaier K, Olson EN, McKinsey TA, and Jin ZG (2007) Angiotensin II stimulates protein kinase D-dependent histone deacetylase 5 phosphorylation and nuclear export leading to vascular smooth muscle cell hypertrophy. Arterioscler Thromb Vasc Biol 27:2355-2362.

Yang Z, Zheng B, Zhang Y, He M, Zhang XH, Ma D, Zhang RN, Wu XL, and Wen JK (2015) miR-155-dependent regulation of mammalian sterile 20-like kinase 2 (MST2) coordinates inflammation, oxidative stress and proliferation in vascular smooth muscle cells. Biochim Biophys Acta 1852:1477-1489.

Yilmaz M, Gangopadhyay SS, Leavis P, Grabarek Z, and Morgan KG (2013) Phosphorylation at $\mathrm{Ser}^{26}$ in the ATP-binding site of $\mathrm{Ca}^{2+} /$ calmodulin-dependent kinase II as a mechanism for switching off the kinase activity. Biosci Rep 33:e00024.

Ying AK, Hassanain HH, Roos CM, Smiraglia DJ, Issa JJ, Michler RE, Caligiuri M, Plass C, and Goldschmidt-Clermont PJ (2000) Methylation of the estrogen receptor-alpha gene promoter is selectively increased in proliferating human aortic smooth muscle cells. Cardiovasc Res 46:172-179.

Yoshida T, Gan Q, and Owens GK (2008) Kruppel-like factor 4, Elk-1, and histone deacetylases cooperatively suppress smooth muscle cell differentiation markers in response to oxidized phospholipids. Am J Physiol Cell Physiol 295 C1175-C1182.

Yu H, Lu Y, Li Z, and Wang Q (2014) microRNA-133: expression, function and therapeutic potential in muscle diseases and cancer. Curr Drug Targets 15: 817-828

Yuen S, Ogut O, and Brozovich FV (2011) MYPT1 protein isoforms are differentially phosphorylated by protein kinase G. J Biol Chem 286:37274-37279.

Yuen SL, Ogut O, and Brozovich FV (2009) Nonmuscle myosin is regulated during smooth muscle contraction. Am J Physiol Heart Circ Physiol 297:H191-H199.

Yuen SL, Ogut O, and Brozovich FV (2014) Differential phosphorylation of LZ+/LZ MYPT1 isoforms regulates MLC phosphatase activity. Arch Biochem Biophys 562: 37-42. 
Yusuf S, Pepine CJ, Garces C, Pouleur H, Salem D, Kostis J, Benedict C, Rousseau M, Bourassa M, and Pitt B (1992) Effect of enalapril on myocardial infarction and unstable angina in patients with low ejection fractions. Lancet 340:1173-1178.

Yusuf S, Pfeffer MA, Swedberg K, Granger CB, Held P, McMurray JJ, Michelson EL Olofsson B, and Ostergren J; CHARM Investigators and Committees (2003) Effects of candesartan in patients with chronic heart failure and preserved left-ventricular ejection fraction: the CHARM-Preserved Trial. Lancet 362:777-781.

Yusuf S, Sleight P, Pogue J, Bosch J, Davies R, and Dagenais G; The Heart Outcomes Prevention Evaluation Study Investigators (2000) Effects of an angiotensinconverting-enzyme inhibitor, ramipril, on cardiovascular events in high-risk patients. N Engl J Med 342:145-153.

Zampetaki A, Attia R, Mayr U, Gomes RS, Phinikaridou A, Yin X, Langley SR, Willeit P, Lu R, and Fanshawe B, et al. (2014) Role of miR-195 in aortic aneurysmal disease. Circ Res 115:857-866.

Zeng W, Yuan JP, Kim MS, Choi YJ, Huang GN, Worley PF, and Muallem S (2008) STIM1 gates TRPC channels, but not Orai1, by electrostatic interaction. Mol Cell 32:439-448.

Zhang H and Fisher SA (2007) Conditioning effect of blood flow on resistance artery smooth muscle myosin phosphatase. Circ Res 100:730-737.
Zhang H, Gu S, Al-Sabeq B, Wang S, He J, Tam A, Cifelli C, Mathalone N, Tirgari S, and Boyd S, et al. (2012) Origin-specific epigenetic program correlates with vascular bed-specific differences in Rgs5 expression. FASEB J 26:181-191.

Zhang J, Herrera AM, Paré PD, and Seow CY (2010) Dense-body aggregates as plastic structures supporting tension in smooth muscle cells. Am J Physiol Lung Cell Mol Physiol 299:L631-L638.

Zhang W, Halligan KE, Zhang X, Bisaillon JM, Gonzalez-Cobos JC, Motiani RK, Hu G, Vincent PA, Zhou J, and Barroso M, et al. (2011) Orai1-mediated I (CRAC) is essential for neointima formation after vascular injury. Circ Res 109:534-542.

Zhang W, Huang Y, Wu Y, and Gunst SJ (2015) A novel role for RhoA GTPase in the regulation of airway smooth muscle contraction. Can J Physiol Pharmacol 93:129-136. Zhao YD, Cai L, Mirza MK, Huang X, Geenen DL, Hofmann F, Yuan JX, and Zhao YY (2012) Protein kinase G-I deficiency induces pulmonary hypertension through Rho A/Rho kinase activation. Am J Pathol 180:2268-2275.

Zhu Y, Bian Z, Lu P, Karas RH, Bao L, Cox D, Hodgin J, Shaul PW, Thoren P, and Smithies O, et al. (2002) Abnormal vascular function and hypertension in mice deficient in estrogen receptor beta. Science 295:505-508.

Zulliger MA and Stergiopulos N (2007) Structural strain energy function applied to the ageing of the human aorta. J Biomech 40:3061-3069. 